Daniela Ramos Truzzi

\title{
Reatividade de ésteres de fósforo(III) em tetraaminas de rutênio.
}

Tese apresentada ao Instituto de Química de São Carlos da Universidade de São Paulo como parte dos requisitos para a obtenção do título de Doutor em Ciência.

Área de concentração: Química Analítica e Inorgânica

Orientador: Prof. Dr. Douglas Wagner Franco

\section{Exemplar revisado}

O exemplar original encontra-se em acervo reservado na Biblioteca do IQSC-USP

São Carlos

2014 
Dedico esta Tese

A meus pais, Doralice e Marcos, que sempre me apoiaram e, mesmo com a distância, sempre se fizeram presentes nesta caminhada.

À minha irmã Renata, que nunca foi apenas uma irmã, mas sim uma grande amiga e companheira de todas as horas.

E ao Juliano pelo amor, carinho e pelas palavras de incentivo e apoio que me ajudaram a seguir em frente na busca desta realização. 
Ao Prof. Dr. Douglas Wagner Franco pela oportunidade de realizar este trabalho e, principalmente, pela atenção e ensinamentos a mim transmitidos.

Aos professores Daniel, Benedito e Ubirajara pelo convívio amigável durante todos estes anos e pelas valiosas discussões.

Ao Thiago, técnico do LQIA, pelas inúmeras discussões, pela pronta disposição em ajudar no que fosse necessário e, sobretudo, por ter se tornado um valioso amigo nesta jornada.

Aos técnicos da Central Analítica de Química (CAQUI), Silvana, Carlinhos, André e Aldimar pela atenção a mim dedicada.

À Veroneide por estar sempre disposta a ajudar demonstrando todo o seu carinho pelos alunos de pós-graduação.

A todos os amigos com quem convivi durante todos estes anos no Laboratório de Química Inorgânica e Analítica e no Laboratório Química de Aguardente do IQSC-USP.

Ao Instituto de Química de São Carlos e à Universidade de São Paulo pela oportunidade.

À CAPES pela bolsa concedida. 
Nas grandes batalhas da vida, o primeiro passo para a vitória é o desejo de vencer.

Mahatma Gandhi

O segredo é não correr atrás das borboletas...

É cuidar do jardim para que elas venham até você.

Mário Quintana 
As alterações na reatividade de ésteres de fósforo(III) promovidas pela coordenação ao centro metálico de rutênio(II) e o mútuo efeito e influência trans entre ésteres de fósforo(III) e ligantes $\pi$-aceptores $\left(\mathrm{NO}^{+}\right.$e $\left.\mathrm{CO}\right)$ foram o foco deste trabalho. Dados de Ressonância Magnética Nuclear adquiridos em função do tempo sugerem que a coordenação de fosfitos ao centro de rutênio(II) estabiliza essas moléculas com respeito à reações de hidrólise e de oxidação. Esta estabilização é maior quando a coordenação se dá no fragmento trans- $\left[\mathrm{Ru}\left(\mathrm{H}_{2} \mathrm{O}\right)\left(\mathrm{NH}_{3}\right)_{4}\right]^{2+}$ do que no trans- $\left[\mathrm{Ru}(\mathrm{NO})\left(\mathrm{NH}_{3}\right)_{4}\right]^{3+}$ devido à menor competição pelos elétrons $4 \mathrm{~d} \pi\left(\mathrm{Ru}^{\mathrm{II}}\right)$ no aqua do que nos nitrosilos complexos. A correlação linear entre os valores numéricos das constantes de hidrólise dos alquil fosfitos nos complexos trans- $\left[\mathrm{Ru}(\mathrm{NO})\left(\mathrm{NH}_{3}\right)_{4} \mathrm{P}(\mathrm{III})\right]^{3+}\left(\mathrm{P}(\mathrm{III})=\mathrm{P}\left(\mathrm{OC}_{3} \mathrm{H}_{7}\right)_{3}, \mathrm{P}\left(\mathrm{OC}_{4} \mathrm{H}_{9}\right)_{3}, \mathrm{P}\left(\mathrm{OC}_{2} \mathrm{H}_{5}\right)_{3}\right.$ e $\left.\mathrm{P}(\mathrm{OH})\left(\mathrm{OC}_{2} \mathrm{H}_{5}\right)_{2}\right)$ e os valores numéricos de $\delta_{13 \mathrm{C}}$ mostram que a hidrólise de fosfitos coordenados a $\mathrm{Ru}(\mathrm{II})$ ocorre preferencialmente via mecanismo de Michaelis Arbusov. Apenas o nitrosilo em que $\mathrm{P}(\mathrm{III})=\mathrm{P}\left(\mathrm{OCH}_{3}\right)_{3}$ não apresentou esta correlação, indicando que, neste caso, provavelmente a hidrólise se dá via mecanismo de Asknes. Os complexos trans- $\left[\mathrm{Ru}(\mathrm{NO})\left(\mathrm{NH}_{3}\right)_{4}\left(\mathrm{P}(\mathrm{O})(\mathrm{OH})_{2}\right)\right] \mathrm{ZnCl}_{4} \quad \mathrm{e} \quad$ trans- $\left[\mathrm{Ru}(\mathrm{CO})\left(\mathrm{NH}_{3}\right)_{4}\left(\mathrm{P}(\mathrm{OH})_{3}\right)\right] \mathrm{ZnCl}_{4} \quad$ foram isolados e caracterizados por Raio-X, UV-vis, RMN, IV, voltametria cíclica e análise elementar. $\mathrm{O} \mathrm{pK}_{\mathrm{a}}$ do ácido fosforoso coordenado foi calculado em solução por meio de espectroscopia de infravermelho apresentando os valores de 0,74 e 3,30 para o nitrosilo e carbonilo complexos, respectivamente. Isto confirma que, em tetraamminas de rutênio(II), o $\mathrm{NO}^{+}$é um recebedor $\pi$ consideravelmente mais forte que o CO. A estabilidade de ambos os complexos em solução aquosa foi acompanhada por UV-vis, ${ }^{31} \mathrm{P}$ RMN e IV. Observou-se que o nitrosônio empresta ao centro metálico de rutênio(II) características de rutênio(III) favorecendo a isomerização do ligante ácido fosforoso, formando as espécies 


\section{Resumo}

trans- $\left[\mathrm{Ru}(\mathrm{NO})\left(\mathrm{NH}_{3}\right)_{4}\left((\mathrm{O}) \mathrm{P}(\mathrm{OH})_{2}\right)\right]^{2+}$ e trans- $\left[\mathrm{Ru}(\mathrm{NO})\left(\mathrm{NH}_{3}\right)_{4}\left((\mathrm{O}) \mathrm{P}(\mathrm{H})(\mathrm{OH})_{2}\right)\right]^{3+}$ e só após isto ocorre a dissociação do ácido fosforoso. Dados experimentais de UV-vis e IV e sua correlação com cálculos DFT, indicam que o CO também induz a isomerização do ácido fosforoso coordenado no íon trans- $\left[\mathrm{Ru}(\mathrm{CO})\left(\mathrm{NH}_{3}\right)_{4}\left(\mathrm{P}(\mathrm{O})(\mathrm{OH})_{2}\right)\right]^{2+}$, porém a velocidade de isomerização é consideravelmente menor do que no nitrosilo complexo. $\mathrm{O}$ composto trans-[Ru(NO)(NH$\left.)_{4}\left(\mathrm{P}(\mathrm{O})\left(\mathrm{OCH}_{2} \mathrm{CH}_{3}\right)_{2}\right)\right]\left(\mathrm{PF}_{6}\right)_{2}$, em que o éster de fósforo é um dialquil fosfito, também foi sintetizado e caracterizado. Os dados cinéticos mostram que o íon trans- $\left[\mathrm{Ru}(\mathrm{NO})\left(\mathrm{NH}_{3}\right)_{4}\left(\mathrm{P}(\mathrm{O})\left(\mathrm{OCH}_{2} \mathrm{CH}_{3}\right)_{2}\right)\right]^{2+}$ é o mais estável dentre os nitrosilos complexos do tipo trans-[Ru(NO)(NH$\left.)_{4} \mathrm{P}(\mathrm{III})\right]^{\mathrm{n}+}$ no que diz respeito às reações de ataque nucleofílico nos ligantes fosfito e nitrosônio, o que o torna um interessante candidato a doador de NO/HNO em meio biológico. 
Changes in phosphorus(III) esters reactivity promoted by coordination to ruthenium(II) metal center and the mutual trans effect and influence of esters of phosphorus(III) and $\pi$-acceptor ligands $\left(\mathrm{NO}^{+}\right.$and $\left.\mathrm{CO}\right)$ were the focus of this work. Nuclear Magnetic Resonance data acquired as function of time suggest that phosphites coordination to ruthenium(II) center stabilizes these molecules regarding to hydrolysis and oxidation reactions. This stabilization is greater when the coordination occurs to

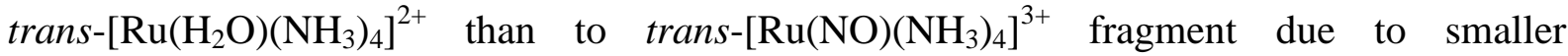
competition for $4 \mathrm{~d} \pi\left(\mathrm{Ru}^{\mathrm{II}}\right)$ electrons in aquo than nitrosyl complexes. The correlation between the numeric values of the alkyl phosphites hydrolysis constants in

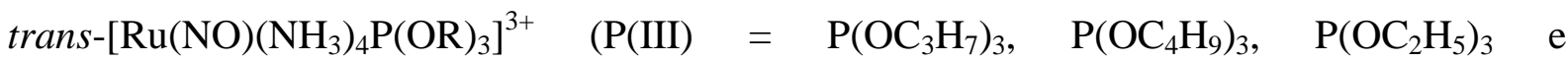
$\left.\mathrm{P}(\mathrm{OH})\left(\mathrm{OC}_{2} \mathrm{H}_{5}\right)_{2}\right)$ complexes and the numeric values of $\delta_{13 \mathrm{C}}$ shows that hydrolysis of phosphites coordinated to $\mathrm{Ru}(\mathrm{II})$ takes place preferably via Michaelis Arbusov mechanism. Only the nitrosyl complex where $\mathrm{P}(\mathrm{III})=\mathrm{P}\left(\mathrm{OCH}_{3}\right)_{3}$ did not exhibit this correlation which indicated that, in this case, the hydrolysis probably occurs via Asknes mechanism. The trans- $\left[\mathrm{Ru}(\mathrm{NO})\left(\mathrm{NH}_{3}\right)_{4}\left(\mathrm{P}(\mathrm{O})(\mathrm{OH})_{2}\right)\right] \mathrm{ZnCl}_{4} \quad$ and $\quad$ trans $-\left[\mathrm{Ru}(\mathrm{CO})\left(\mathrm{NH}_{3}\right)_{4}\left(\mathrm{P}(\mathrm{OH})_{3}\right)\right] \mathrm{ZnCl}_{4}$ complexes were isolated and characterized using X-ray, UV-vis, NMR, IR, elemental analysis, and cyclic voltammetry. The $\mathrm{pK}_{\mathrm{a}}$ of the coordinated phosphorous acid was calculated in solution through infrared spectroscopy and exhibited the values of 0.74 and 3.30 for nitrosyl and carbonyl complexes, respectively. This confirm that, in ruthenium(II) tetraammines, $\mathrm{NO}^{+}$is a stronger $\pi$-acceptor than $\mathrm{CO}$. The stability of these both complexes in aqueous solution was followed by UV-vis, ${ }^{31} \mathrm{P}$ NMR and IR. It was observed that nitrosonium ligand makes the ruthenium(II) metal center exhibit ruthenium(III) characteristics favoring the isomerization of the phosphorous acid ligand leading to trans- $\left[\mathrm{Ru}(\mathrm{NO})\left(\mathrm{NH}_{3}\right)_{4}\left((\mathrm{O}) \mathrm{P}(\mathrm{OH})_{2}\right)\right]^{2+}$ 
and trans-[Ru(NO) $\left.\left(\mathrm{NH}_{3}\right)_{4}\left((\mathrm{O}) \mathrm{P}(\mathrm{H})(\mathrm{OH})_{2}\right)\right]^{3+}$ species, and only after that occurs the dissociation of the phosphorous acid. UV-vis and IR experimental data and the correlation with DFT calculations indicate that $\mathrm{CO}$ also induces isomerization of the coordinated phosphorous acid in trans- $\left[\mathrm{Ru}(\mathrm{CO})\left(\mathrm{NH}_{3}\right)_{4}\left(\mathrm{P}(\mathrm{O})(\mathrm{OH})_{2}\right)\right]^{2+}$, but the isomerization rate is considerably smaller than in the nitrosyl complex. The trans- $\left[\mathrm{Ru}(\mathrm{NO})\left(\mathrm{NH}_{3}\right)_{4}\left(\mathrm{P}(\mathrm{O})\left(\mathrm{OCH}_{2} \mathrm{CH}_{3}\right)_{2}\right)\right]\left(\mathrm{PF}_{6}\right)_{2}$ compound, wherein the phosphorus ester is a dialkyl phosphite, was also synthesized and characterized. The kinetic data show that the trans- $\left[\mathrm{Ru}(\mathrm{NO})\left(\mathrm{NH}_{3}\right)_{4}\left(\mathrm{P}(\mathrm{O})\left(\mathrm{OCH}_{2} \mathrm{CH}_{3}\right)_{2}\right)\right]^{2+}$ is the most stable among the nitrosyl complexes of the trans-[Ru(NO) $\left.\left(\mathrm{NH}_{3}\right)_{4} \mathrm{P}(\mathrm{III})\right]^{\mathrm{n}+}$ type regarding to the phosphite and nitrosonium nucleophilic attack reactions which makes this complex an interesting candidate as a NO/HNO-donor in biological medium. 
Figura 1 - Tetraaminas de rutênio.

Figura 2 - Mecanismos de reação propostos para a hidrólise de fosfitos (a) mecanismo de Michaelis-Arbusov, (b) mecanismo proposto por Aksnes (similar a ésteres orgânicos) (c) catálise ácida via mecanismo de Michaelis-Arbusov e (d) catálise ácida via mecanismo proposto por Aksnes. $\mathrm{O}^{*}={ }^{18 / 17} \mathrm{O}$.

Figura 3 - Representação ORTEP-3 da estrutura trans-[Ru(NO) $\left.\left(\mathrm{NH}_{3}\right)_{4}\left(\mathrm{P}(\mathrm{O})(\mathrm{OH})_{2}\right)\right] \mathrm{ZnCl}_{4}$ com elipsoides de deslocamentos com probabilidades de $50 \%$.

Figura 4 - Esquema proposto inicialmente para da dissociação do ligante de ácido fosforoso do fragmento trans- $\left[\mathrm{Ru}(\mathrm{NO})\left(\mathrm{NH}_{3}\right)_{4}\right]^{3+}$

Figura 5 - Gráfico de energia $\left(\mathrm{kcal} \mathrm{mol}^{-1}\right)$ por coordenada intrínseca de reação (IRC) para a reação de isomerização do íon trans- $\left[\mathrm{Ru}(\mathrm{NO})\left(\mathrm{NH}_{3}\right)_{4}\left(\mathrm{P}(\mathrm{O})(\mathrm{OH})_{2}\right)\right]^{2+}$

Figura 6 - Dissociação do ligante de ácido fosforoso a partir do complexo trans- $\left[\mathrm{Ru}(\mathrm{NO})\left(\mathrm{NH}_{3}\right)_{4}\left(\mathrm{P}(\mathrm{OH})_{3}\right)\right]^{3+}$

Figura 7 - Espectro de infravermelho do íon trans- $\left[\mathrm{Ru}(\mathrm{NO})\left(\mathrm{NH}_{3}\right)_{4}\left(\mathrm{P}(\mathrm{OH})_{3}\right)\right]^{3+}$ em solução $\mathrm{pH}$ $=3,0, \mu=0,10 \mathrm{~mol} \mathrm{~L}^{-1}, \mathrm{C}_{\mathrm{Ru}}=5,0 \times 10^{-2} \mathrm{~mol} \mathrm{~L}^{-1}$ e espaçador de $0,10 \mathrm{~mm}$. Linha pontilhada: $\mathrm{t}=5$ min. Linha cheia: $\mathrm{t}=1 \mathrm{~h}$

Figura 8 - (a) Espectro eletrônico experimental do íon trans- $\left[\mathrm{Ru}(\mathrm{NO})\left(\mathrm{NH}_{3}\right)_{4}\left(\mathrm{P}(\mathrm{OH})_{3}\right)\right]^{3+}$ em solução $\mathrm{pH}=3,0, \mu=0,10 \mathrm{~mol} \mathrm{~L}{ }^{-1}, 25 \pm 0,1^{\circ} \mathrm{C}$ e $\mathrm{C}_{\mathrm{Ru}}=3,70 \times 10^{-4} \mathrm{~mol} \mathrm{~L}^{-1}$ com intervalo de tempo de 5 a 20 minutos (Espectro inserido: 20 a 30 minutos); (b) Espectro eletrônico teórico para cada um dos nitrosilos complexos de ácido fosforoso. 48

Figura 9 - Espectro eletrônico do íon trans- $\left[\mathrm{Ru}(\mathrm{NO})\left(\mathrm{NH}_{3}\right)_{4}\left(\mathrm{P}(\mathrm{OH})\left(\mathrm{OCH}_{2} \mathrm{CH}_{3}\right)_{2}\right)\right]^{3+}$ em solução pH 1,0, $\mu=0,10 \mathrm{~mol} \mathrm{~L}^{-1} \mathrm{CF}_{3} \mathrm{COONa}, 25 \pm 0,1^{\circ} \mathrm{C}$ (a) $\mathrm{C}_{\mathrm{Ru}}=2,08 \times 10^{-4} \mathrm{~mol} \mathrm{~L}^{-1} \mathrm{e}$ cubeta de 1,0 cm de caminho ótico, (b) $\mathrm{C}_{\mathrm{Ru}}=1,28 \times 10^{-3} \mathrm{~mol} \mathrm{~L}^{-1}$ e cubeta de $5,0 \mathrm{~cm}$ de caminho ótico.

Figura 10 - (a) Voltamograma cíclico para o íon trans- $\left[\mathrm{Ru}(\mathrm{NO})\left(\mathrm{NH}_{3}\right)_{4}\left(\mathrm{P}(\mathrm{OH})\left(\mathrm{OCH}_{2} \mathrm{CH}_{3}\right)_{2}\right)\right]^{3+}$ em meio aquoso $\mathrm{pH} 1,0 ; \mu=0,1 \mathrm{~mol} \mathrm{~L}^{-1} ; \mathrm{C}_{\mathrm{Ru}}=1,5 \times 10^{-3} \mathrm{~mol} \mathrm{~L}^{-1} ; \mathrm{T}=5 \pm 0,1^{\circ} \mathrm{C}$; velocidade $=$ $100 \mathrm{mV} \mathrm{s}^{-1}$. (b) Voltamograma de pulso diferencial realizado a velocidade de $20 \mathrm{mV} \mathrm{s}^{-1}$......54

Figura 11 - Gráfico de k. $\tau$ versus $\tau$ para $\mathrm{o}$ íon complexo trans- $\left[\mathrm{Ru}(\mathrm{NO})\left(\mathrm{NH}_{3}\right)_{4} \mathrm{P}(\mathrm{O})\left(\mathrm{OCH}_{2} \mathrm{CH}_{3}\right)_{2}\right]^{2+} \mathrm{em} \mathrm{pH} 2,0 ; \mu=0,1 \mathrm{~mol} \mathrm{~L}^{-1} ; \mathrm{C}_{\mathrm{Ru}}=1,5 \times 10^{-3} \mathrm{~mol} \mathrm{~L}^{-}$ ${ }^{1}$ e T $=25 \pm 0,1^{\circ} \mathrm{C}$ (a) Potencial de inversão $\left(\mathrm{E}_{\lambda}\right)=-0,60 \mathrm{~V}$ vs $\mathrm{ECS}$; (b) $\mathrm{E}_{\lambda}=-0,62 \mathrm{~V}$ vs ECS55 Figura 12 - Espectro na região do infravermelho do composto trans-[Ru(NO) $\left.\left(\mathrm{NH}_{3}\right)_{4}\left(\mathrm{P}(\mathrm{O})\left(\mathrm{OCH}_{2} \mathrm{CH}_{3}\right)_{2}\right)\right]\left(\mathrm{PF}_{6}\right)_{2}$ em pastilha de $\mathrm{KBr}$. 56

Figura 13 - Espectro de infravermelho: (a) Experimental para $o$ íon trans- $\left[\mathrm{Ru}(\mathrm{NO})\left(\mathrm{NH}_{3}\right)_{4}\left(\mathrm{P}(\mathrm{OH})\left(\mathrm{OCH}_{2} \mathrm{CH}_{3}\right)_{2}\right)\right]^{3+}$ em solução $\mathrm{pH} 1$,5; (b) Teórico para o íon trans- $\left[\mathrm{Ru}(\mathrm{NO})\left(\mathrm{NH}_{3}\right)_{4}\left(\mathrm{P}(\mathrm{OH})\left(\mathrm{OCH}_{2} \mathrm{CH}_{3}\right)_{2}\right)\right]^{3+}$ e (c) Teórico para $\mathrm{o}$ íon

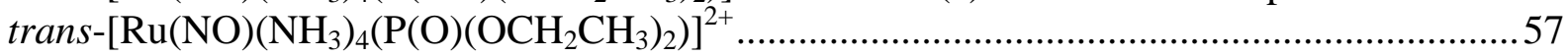




\section{Lista de Figuras}

Figura 14 - Distribuição dos produtos em função do tempo obtido a partir dos dados cinéticos de ${ }^{31} \mathrm{P}$ RMN do íon trans- $\left[\mathrm{Ru}(\mathrm{NO})\left(\mathrm{NH}_{3}\right)_{4}\left(\mathrm{P}(\mathrm{O})\left(\mathrm{OCH}_{2} \mathrm{CH}_{3}\right)_{2}\right)\right]^{2+}$ em pH 7,5 e $25 \pm 0,5^{\circ} \mathrm{C}$; (a) $\delta_{31 \mathrm{P}}=68 \mathrm{ppm}$; trans- $\left[\mathrm{Ru}(\mathrm{NO})\left(\mathrm{NH}_{3}\right)_{4}\left(\mathrm{P}(\mathrm{O})\left(\mathrm{OCH}_{2} \mathrm{CH}_{3}\right)_{2}\right)\right]^{2+}$;

Figura 15 - Espectro de UV-vis para o íon trans- $\left[\mathrm{Ru}(\mathrm{NO})\left(\mathrm{NH}_{3}\right)_{4}\left(\mathrm{P}(\mathrm{O})\left(\mathrm{OCH}_{2} \mathrm{CH}_{3}\right)_{2}\right)\right]^{2+}$ na presença de excesso de pirazina em pH 7,5 e $25 \pm 0,1^{\circ} \mathrm{C}$. Inserção: Curva de $\ln \left(\mathrm{A}-\mathrm{A}_{\mathrm{t}}\right)$ versus tempo (s)

Figura 16 - Espectro de infravermelho para o trans- $\left[\mathrm{Ru}(\mathrm{NO})\left(\mathrm{NH}_{3}\right)_{4} \mathrm{P}(\mathrm{O})\left(\mathrm{OCH}_{2} \mathrm{CH}_{3}\right)_{2}\right]^{2+} \mathrm{em}$ pH 7,5 (tampão TRIS na concentração de $0,20 \mathrm{~mol} \mathrm{~L}^{-1}$ ), $\mathrm{C}_{\mathrm{Ru}}=7,8 \times 10^{-2} \mathrm{~mol} \mathrm{~L}^{-1}$ e espaçador de $0,05 \mathrm{~mm}$

Figura 17 - (a) Voltamograma cíclico para o íon trans- $\left[\mathrm{Ru}(\mathrm{NO})\left(\mathrm{NH}_{3}\right)_{4}\left(\mathrm{P}(\mathrm{O})\left(\mathrm{OCH}_{2} \mathrm{CH}_{3}\right)_{2}\right)\right]^{2+}$ em solução $\mathrm{pH} 7,5, \mu=0,1 \mathrm{~mol} \mathrm{~L}^{-1}, \mathrm{C}_{\mathrm{Ru}}=1,5 \times 10^{-3} \mathrm{~mol} \mathrm{~L}^{-1}, \mathrm{~T}=25 \pm 0,1{ }^{\circ} \mathrm{C}$, velocidade $=$ $100 \mathrm{mV} \mathrm{s}^{-1}$; (b) Voltamograma de pulso diferencial realizado em velocidade de $20 \mathrm{mV} \mathrm{s}^{-1}$ e $\mathrm{T}=5 \pm 0,1^{\circ} \mathrm{C}$

Figura 18 - Ciclo catalítico para a conversão de nitrito a óxido nítrico por meio de complexos de rutênio.

Figura 19 - Espectro de ${ }^{1} \mathrm{H}$ RMN para o dietil fosfito em $\mathrm{D}_{2} \mathrm{O}$ e $\mathrm{pH}=3,0\left(\mathrm{CD}_{3} \mathrm{COOD}\right)$ após 20 horas em solução, $\mathrm{C}_{\mathrm{P}(\mathrm{OH})(\mathrm{OEt}) 2}=2,0 \times 10^{-4} \mathrm{~mol} \mathrm{~L}^{-1}$ e $25 \pm 0,5{ }^{\circ} \mathrm{C}$; (a) dietil fosfito, (b) monoetil fosfito e (c) etanol

Figura 20 - trans- $\left[\mathrm{Ru}(\mathrm{NO})\left(\mathrm{NH}_{3}\right)_{4}\left(\mathrm{P}\left(\mathrm{OCH}_{3}\right)_{3}\right)\right]^{3+}$ em $\mathrm{D}_{2} \mathrm{O}, \mathrm{pH}=3,0\left(\mathrm{CD}_{3} \mathrm{COOD}\right)$ e $25 \pm 0,5{ }^{\circ} \mathrm{C}$ (a) Espectro de ${ }^{31} \mathrm{P} \mathrm{RMN}$ ( $\mathrm{NH}_{4} \mathrm{PF}_{6}$ como referência interna); (b).Variação da área dos picos de ${ }^{31} \mathrm{P}$ RMN em função do tempo. 74

Figura 21 - Espectro de infravermelho para o trans- $\left[\mathrm{Ru}(\mathrm{NO})\left(\mathrm{NH}_{3}\right)_{4}\left(\mathrm{P}\left(\mathrm{OCH}_{3}\right)_{3}\right)\right]^{3+}$ em pH 3,0, $\mu=0,1 \mathrm{~mol} \mathrm{~L}^{-1}, 25 \pm 1,0{ }^{\circ} \mathrm{C}$.

Figura 22 - Correlação entre os valores de $k_{\text {hid }}$ e $\delta_{13 \mathrm{C}}(\mathrm{ppm})$ em nitrosilos complexos de rutênio 78

Figura 23 - Mecanismo de hidrólise de fosfitos em nitrosilos complexos de rutênio (a) Quebra da ligação O-C (Mecanismo de Michaelis-Arbusov), (b) Quebra da ligação P-O (Mecanismo de Aksnes)

Figura 24 - trans- $\left[\mathrm{Ru}(\mathrm{NO})\left(\mathrm{NH}_{3}\right)_{4}\left(\mathrm{P}\left(\mathrm{OCH}_{3}\right)_{3}\right)\right]^{3+}$ em meio aquoso $\mathrm{pH} 2,0, \mu=0,1 \mathrm{~mol} \mathrm{~L}^{-1}$, $\mathrm{C}_{\mathrm{Ru}}=1,5 \times 10^{-3} \mathrm{~mol} \mathrm{~L}^{-1}, \mathrm{~T}=5 \pm 0,1^{\circ} \mathrm{C}$ (a) Voltamograma cíclico, $\mathrm{v}=100 \mathrm{mV} \mathrm{s}{ }^{-1}$; (b) Voltamograma de pulso diferencial, $\mathrm{v}=20 \mathrm{mV} \mathrm{s}^{-1}$.

$\begin{array}{lllll}\text { Figura } & 25 & - & \text { Representação } & \text { ORTEP-3 }\end{array}$ trans-[Ru(CO) $\left.\left(\mathrm{NH}_{3}\right)_{4}\left(\mathrm{P}(\mathrm{OH})_{3}\right)\right]\left(\mathrm{ZnCl}_{4}\right) \cdot \mathrm{H}_{2} \mathrm{O}$ com elipsoides de deslocamentos que apresentam probabilidades de $40 \%$ 


\section{Lista de Figuras}

Figura 26 - Espectro eletrônico do íon trans- $\left[\mathrm{Ru}(\mathrm{CO})\left(\mathrm{NH}_{3}\right)_{4}\left(\mathrm{P}(\mathrm{OH})_{3}\right)\right]^{2+}$ em solução pH 2,0, $\mu=0,1 \mathrm{~mol} \mathrm{~L}^{-1} \mathrm{CF}_{3} \mathrm{COONa}$, temperatura de $25 \pm 0,1^{\circ} \mathrm{C}$ e cubeta de $1,0 \mathrm{~cm}$ de caminho ótico, (a) $\mathrm{C}_{\mathrm{Ru}}=4,0 \times 10^{-4} \mathrm{~mol} \mathrm{~L}^{-1}$ e (b) $\mathrm{C}_{\mathrm{Ru}}=1,0 \times 10^{-3} \mathrm{~mol} \mathrm{~L}^{-1}$

Figura 27 - Voltamograma cíclico do composto trans-[Ru(CO) $\left.\left(\mathrm{NH}_{3}\right)_{4}\left(\mathrm{P}(\mathrm{O})(\mathrm{OH})_{2}\right)\right](\mathrm{Cl})$ em solução pH 2,0, $\mu=0,1 \mathrm{molL}^{-1} \mathrm{CF}_{3} \mathrm{COONa}, \mathrm{v}=50 \mathrm{mVs}^{-1}$ e $\mathrm{C}_{\mathrm{Ru}}=1,0 \times 10^{-3} \mathrm{~mol} \mathrm{~L}^{-1}$, $25 \pm 0,1^{\circ} \mathrm{C},(-)$ primeiro ciclo (-) segundo ciclo

Figura 28 - Espectro na região do infravermelho do composto trans- $\left[\mathrm{Ru}(\mathrm{CO})\left(\mathrm{NH}_{3}\right)_{4}\left(\mathrm{P}(\mathrm{O})(\mathrm{OH})_{2}\right)\right](\mathrm{Cl})$ em pastilha de $\mathrm{KBr}$.

Figura 29 - Deconvolução das bandas de $\mathrm{CO}$ referente aos complexos trans- $\left[\mathrm{Ru}(\mathrm{CO})\left(\mathrm{NH}_{3}\right)_{4}\left(\mathrm{P}(\mathrm{OH})_{3}\right)\right]^{2+} /$ trans $-\left[\mathrm{Ru}(\mathrm{CO})\left(\mathrm{NH}_{3}\right)_{4}\left(\mathrm{P}(\mathrm{O})(\mathrm{OH})_{2}\right)\right]^{+}$em pH 3,0.............. 90

Figura 30 - Sigmoidal obtida por meio da área da banda de $\mathrm{CO} \mathrm{em} 1868 \mathrm{~cm}^{-1}$, referente ao íon trans- $\left[\mathrm{Ru}(\mathrm{CO})\left(\mathrm{NH}_{3}\right)_{4}\left(\mathrm{P}(\mathrm{O})(\mathrm{OH})_{2}\right)\right]^{+}$, em diferentes concentrações hidrogeniônicas, $\mathrm{pK}_{\mathrm{a}}=3,30$. 92

Figura $31-{ }^{31} \mathrm{P}$ RMN do complexo trans- $\left[\mathrm{Ru}(\mathrm{CO})\left(\mathrm{NH}_{3}\right)_{4}\left(\mathrm{P}(\mathrm{O})(\mathrm{OH})_{2}\right)\right](\mathrm{Cl}), \mathrm{C}_{\mathrm{Ru}}=2,0 \times 10^{-2}$ mol L ${ }^{-1}, \mathrm{NH}_{4} \mathrm{PF}_{6}$ como padrão interno $\left(\delta=-144 \mathrm{ppm}\right.$ ), temperatura de $25 \pm 0,5^{\circ} \mathrm{C}$, (a) solução pH 7,5 (TRIS), (b) Após adição de $\mathrm{CF}_{3} \mathrm{COOH} \mathrm{6,0} \mathrm{mol} \mathrm{L}^{-1}, \mathrm{pH}$ 1,0.

Figura 32 - Espectros eletrônico do íon trans- $\left[\mathrm{Ru}(\mathrm{CO})\left(\mathrm{NH}_{3}\right)_{4}\left(\mathrm{P}(\mathrm{O})(\mathrm{OH})_{2}\right)\right]^{+}$em função do tempo em solução pH 7,5 (Tampão TRIS), $\mu=0,1 \mathrm{~mol} \mathrm{~L}^{-1} \mathrm{CF}_{3} \mathrm{COONa}, 25 \pm 0,1^{\circ} \mathrm{C}$, cubeta de $1,0 \mathrm{~cm}$ de caminho ótico e $\mathrm{C}_{\mathrm{Ru}}=4,0 \times 10^{-4} \mathrm{~mol} \mathrm{~L}^{-1}$

Figura 33 - Espectro na região do infravermelho do íon trans- $\left[\mathrm{Ru}(\mathrm{CO})\left(\mathrm{NH}_{3}\right)_{4}\left(\mathrm{P}(\mathrm{O})(\mathrm{OH})_{2}\right)\right]^{+}$ em solução pH 7,5 após 1 minuto e após 72 horas, obtido em pastilhas de silício, com espaçador de teflon de $0,10 \mathrm{~mm}$ e $\mathrm{C}_{\mathrm{Ru}}=5,0 \times 10^{-2} \mathrm{~mol} \mathrm{~L}^{-1}$.

Figura 34 - Valores teóricos calculados para a frequência de estiramento $\mathrm{CO}$ e carga de Mulliken para o átomo de fósforo para as possíveis estruturas do carbonilo complexo de ácido fosforoso

Figura 35 - Espectro de infravermelho teórico para os carbonilos complexos de ácido fosforoso: trans- $\left[\mathrm{Ru}(\mathrm{CO})\left(\mathrm{NH}_{3}\right)_{4}\left(\mathrm{P}(\mathrm{O})(\mathrm{OH})_{3}\right)\right]^{+}$e trans- $\left[\mathrm{Ru}(\mathrm{CO})\left(\mathrm{NH}_{3}\right)_{4}\left((\mathrm{O}) \mathrm{P}(\mathrm{H})(\mathrm{OH})_{3}\right)\right]^{2+} . .98$

Figura 36 - Espectro eletrônico teórico (TD-DFT) para os carbonilos complexos de ácido fosforoso: trans- $\left[\mathrm{Ru}(\mathrm{CO})\left(\mathrm{NH}_{3}\right)_{4}\left(\mathrm{P}(\mathrm{O})(\mathrm{OH})_{3}\right)\right]^{+}, \quad$ trans- $\left[\mathrm{Ru}(\mathrm{CO})\left(\mathrm{NH}_{3}\right)_{4}\left((\mathrm{O}) \mathrm{P}(\mathrm{OH})_{2}\right)\right]^{+} \quad \mathrm{e}$

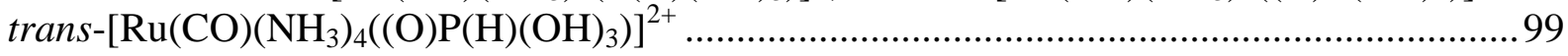

Figura 37 - Dissociação do ligante de ácido fosforoso a partir do complexo trans $-\left[\mathrm{Ru}(\mathrm{CO})\left(\mathrm{NH}_{3}\right)_{4}\left(\mathrm{P}(\mathrm{OH})_{3}\right)\right]^{2+}$ 


\section{Lista de tabelas}

Tabela 1 - Dados cristalográficos para o trans-[Ru(NO)(NH$\left.)_{4}\left(\mathrm{P}(\mathrm{O})(\mathrm{OH})_{2}\right)\right] \mathrm{ZnCl}_{4}$ 40

Tabela 2 - Distâncias e ângulos de ligação para 0 complexo trans-[Ru(NO) $\left.\left(\mathrm{NH}_{3}\right)_{4}\left(\mathrm{P}(\mathrm{O})(\mathrm{OH})_{2}\right)\right] \mathrm{ZnCl}_{4}$

Tabela 3 - Cargas atômicas de Mulliken calculadas para os complexos de ácido fosforoso ... 46

Tabela 4 - Dados de Análise Elementar 51

Tabela 5 - Principais bandas e as atribuições propostas 57

Tabela 6 - Deslocamentos químico para o íon trans- $\left[\mathrm{Ru}(\mathrm{NO})\left(\mathrm{NH}_{3}\right)_{4}\left(\mathrm{P}(\mathrm{O})\left(\mathrm{OCH}_{2} \mathrm{CH}_{3}\right)_{2}\right)\right]^{2+} \mathrm{em}$ $\mathrm{D}_{2} \mathrm{O}$ (pH 3,0, $\mathrm{CD}_{3} \mathrm{COOD}$ ) utilizando como referências internas o TMSP-D4 e $\mathrm{NH}_{4} \mathrm{PF}_{6}$ 58

Tabela 7 - Constante de velocidade de hidrólise para trialquil fosfitos coordenados ao fragmento trans- $\left[\mathrm{Ru}(\mathrm{NO})\left(\mathrm{NH}_{3}\right)_{4}\right]^{3+}$ em pH 3,0 e $25,0 \pm 0,5^{\circ} \mathrm{C}$ 76

Tabela 8 - Dados eletroquímicos e $\mathrm{k}_{-\mathrm{NO}}$ para íons complexos do tipo trans- $\left[\mathrm{Ru}(\mathrm{NO})\left(\mathrm{NH}_{3}\right)_{4} \mathrm{P}(\mathrm{III})\right]^{3+}$ em $\mathrm{pH} 2,0$ e $25,0 \pm 0,1^{\circ} \mathrm{C}$ .81

Tabela 9 - Dados de Análise Elementar e Espectrometria de Emissão por Plasma para o trans $-\left[\mathrm{Ru}(\mathrm{CO})\left(\mathrm{NH}_{3}\right)_{4}\left(\mathrm{P}(\mathrm{O})(\mathrm{OH})_{2}\right)\right](\mathrm{Cl})$ 83

Tabela 10 - Dados cristalográficos para o trans- $\left[\mathrm{Ru}(\mathrm{CO})\left(\mathrm{NH}_{3}\right)_{4}\left(\mathrm{P}(\mathrm{OH})_{3}\right)\right]\left(\mathrm{ZnCl}_{4}\right) \cdot \mathrm{H}_{2} \mathrm{O}$ 84

Tabela 11 - Distâncias e ângulos de ligação para o complexo trans- $\left[\mathrm{Ru}(\mathrm{CO})\left(\mathrm{NH}_{3}\right)_{4}\left(\mathrm{P}(\mathrm{OH})_{3}\right)\right]\left(\mathrm{ZnCl}_{4}\right) \cdot \mathrm{H}_{2} \mathrm{O}$ 85

Tabela 12 - Principais bandas e as atribuições propostas 89 
NO - Óxido nítrico

$\mathrm{NO}^{-}$- Nitroxila

$\mathrm{NO}^{+}$- Nitrosônio

CO - Monóxido de carbono

$\mathrm{H}_{2} \mathrm{~S}$ - Sulfeto de hidrogênio

VC - Voltametria cíclica

VPD - Voltametria de pulso diferencial

$\mathrm{E}_{1 / 2}$ - Potencial de meia-onda

$\mathrm{E}_{\lambda}$ - Potencial de inversão

$\tau$ - Intervalo de tempo (seg) entre $E_{1 / 2}$ e $E_{\lambda}$

Epa - Potencial de pico anódico

Epc - Potencial de pico catódico

$\mathrm{i}_{\mathrm{pa}}$ - Corrente de pico anódico

$\mathrm{i}_{\mathrm{pc}}$ - Corrente de pico catódico

ECS - Eletrodo de calomelano saturado

k-NO - Constante de velocidade de liberação de NO

$\mathrm{P}\left(\mathrm{OC}_{2} \mathrm{H}_{5}\right)_{3}$ - Trietil fosfito

$\mathrm{P}\left(\mathrm{OCH}_{3}\right)_{3}$ - Trimetil fosfito

$\mathrm{P}\left(\mathrm{O}^{\mathrm{i}} \mathrm{C}_{3} \mathrm{H}_{7}\right)_{3}$ - Tri-isopropil fosfito

$\mathrm{P}\left(\mathrm{OC}_{4} \mathrm{H}_{9}\right)_{3}$ - Tributil fosfito

$\mathrm{P}(\mathrm{OH})\left(\mathrm{OC}_{2} \mathrm{H}_{5}\right)_{2}$ - Dietil fosfito

$\mathrm{P}(\mathrm{OH})_{3}$ - Ácido fosforoso

$v$ - Frequência de estiramento

$\delta$ - Deslocamento químico de RMN

$\lambda$ - Comprimento de onda

$\varepsilon$ - Absortividade molar

$\mathrm{W}_{1 / 2}$ - Largura de banda a meia altura

DFT - Teoria do Funcional da Densidade

TD-DFT - Teoria do Funcional da Densidade Dependente do Tempo

QST3 - Synchronous Transit-Guided Quasi-Newton approach

IRC - Coordenada intrínseca de reação

$\mathrm{E}_{\text {rel }}$ - Energia relativa

TS - Estado de transição

$\mathrm{E}_{\mathrm{a}}$ - Energia de ativação 


\section{Sumário}

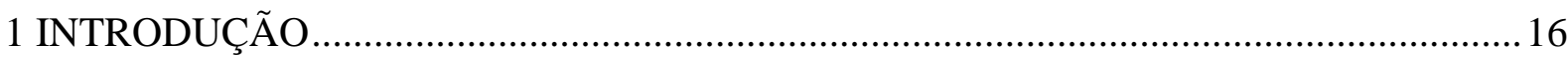

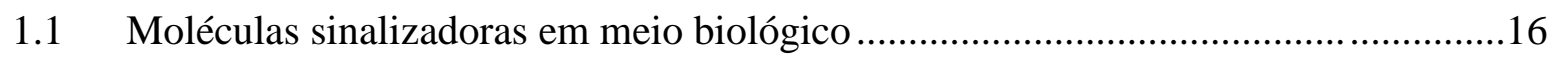

1.2 Complexos metálicos como carregadores de fármacos .......................................... 18

1.3 Ligantes de fósforo(III): estabilidade, reatividade química e efeito e influência

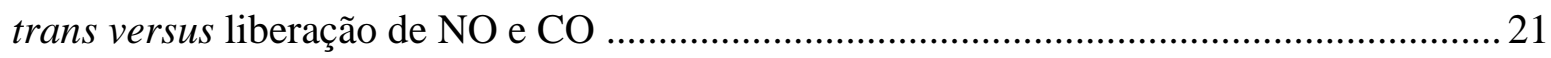

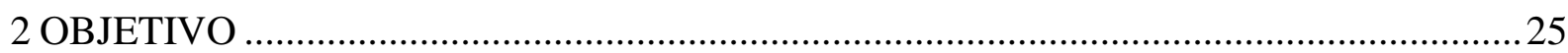

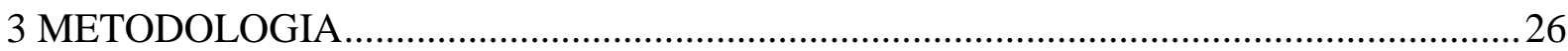

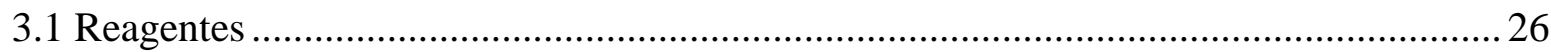

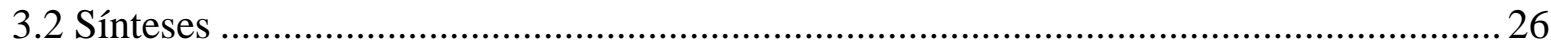

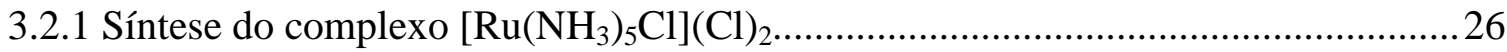

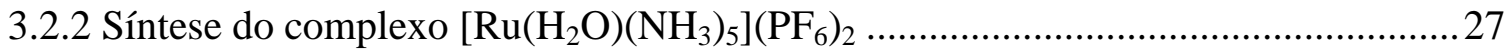

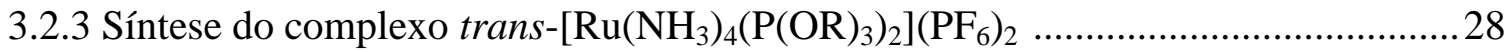

3.2.4 Síntese dos complexos trans- $\left[\mathrm{Ru}\left(\mathrm{H}_{2} \mathrm{O}\right)\left(\mathrm{NH}_{3}\right)_{4} \mathrm{P}(\mathrm{OR})_{3}\right]\left(\mathrm{PF}_{6}\right)_{2} \quad$ e trans- $\left[\mathrm{Ru}(\mathrm{NO})\left(\mathrm{NH}_{3}\right)_{4} \mathrm{P}(\mathrm{OR})_{3}\right]\left(\mathrm{PF}_{6}\right)_{3}, \quad \mathrm{P}(\mathrm{OR})_{3}=\mathrm{P}\left(\mathrm{OCH}_{3}\right)_{3}, \quad \mathrm{P}\left(\mathrm{OC}_{2} \mathrm{H}_{5}\right), \quad \mathrm{P}\left(\mathrm{O}^{\mathrm{i}} \mathrm{C}_{3} \mathrm{H}_{7}\right)_{3} \quad \mathrm{e}$

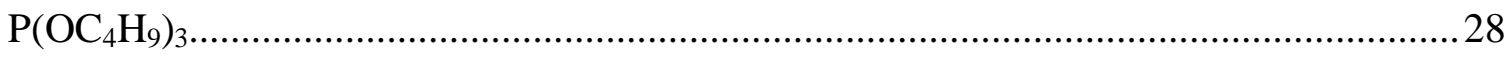

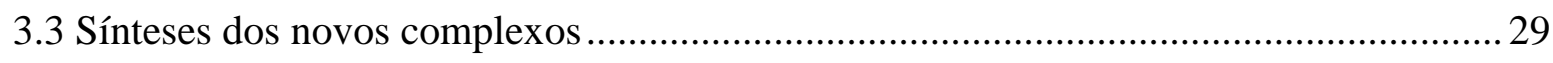

3.3.1 Síntese do complexo trans- $\left[\mathrm{Ru}(\mathrm{NO})\left(\mathrm{NH}_{3}\right)_{4} \mathrm{P}(\mathrm{O})(\mathrm{OH})_{2}\right]\left(\mathrm{ZnCl}_{4}\right) \cdot 3 \mathrm{H}_{2} \mathrm{O}$..................29

3.3.2 Síntese do complexo trans- $\left[\mathrm{Ru}(\mathrm{CO})\left(\mathrm{NH}_{3}\right)_{4} \mathrm{P}(\mathrm{O})(\mathrm{OH})_{2}\right](\mathrm{X})$ em que $\mathrm{X}=\mathrm{ZnCl}_{4}{ }^{2-}$

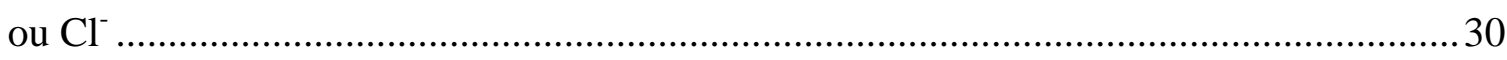

3.3.3 Síntese do complexo trans- $\left[\mathrm{Ru}\left(\mathrm{H}_{2} \mathrm{O}\right)\left(\mathrm{NH}_{3}\right)_{4} \mathrm{P}(\mathrm{OH})\left(\mathrm{OCH}_{2} \mathrm{CH}_{3}\right)_{2}\right]\left(\mathrm{PF}_{6}\right)_{2} \ldots \ldots \ldots \ldots \ldots . . . . . . .30$

3.3.4 Síntese do complexo trans- $\left[\mathrm{Ru}(\mathrm{NO})\left(\mathrm{NH}_{3}\right)_{4} \mathrm{P}(\mathrm{O})\left(\mathrm{OCH}_{2} \mathrm{CH}_{3}\right)_{2}\right]\left(\mathrm{PF}_{6}\right)_{2} \ldots \ldots \ldots \ldots \ldots . . . . . . . . . .31$

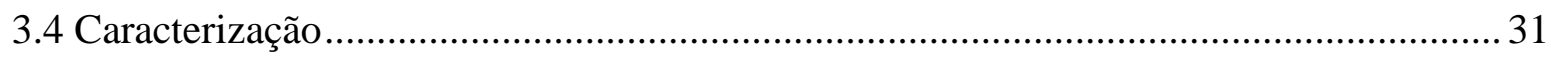

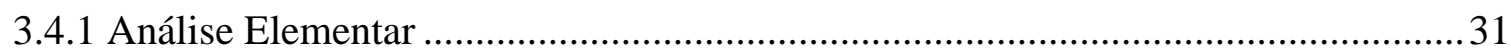

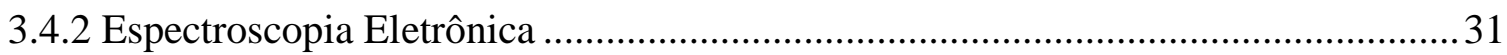

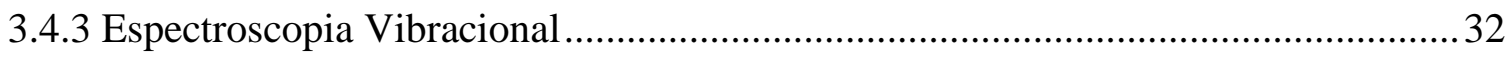

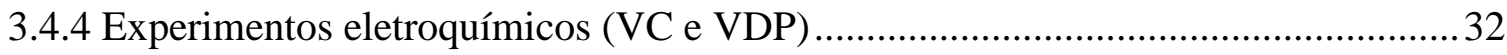

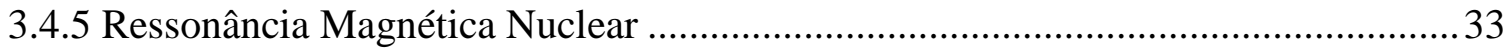

3.4.6 Difração de raios-X e determinação de estrutura ...................................................... 34

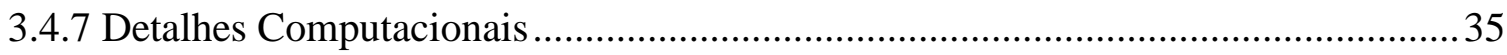

3.5 Hidrólise dos $\mathrm{P}(\mathrm{OR})_{3}=\mathrm{P}\left(\mathrm{OCH}_{3}\right)_{3}, \quad \mathrm{P}\left(\mathrm{OC}_{2} \mathrm{H}_{5}\right), \quad \mathrm{P}\left(\mathrm{O}^{\mathrm{i}} \mathrm{C}_{3} \mathrm{H}_{7}\right)_{3}, \quad \mathrm{P}\left(\mathrm{OC}_{4} \mathrm{H}_{9}\right)_{3} \quad$ e $\mathrm{P}(\mathrm{OH})\left(\mathrm{OC}_{2} \mathrm{H}_{5}\right)_{2}$ livres e coordenados aos fragmentos trans- $\left[\mathrm{Ru}\left(\mathrm{H}_{2} \mathrm{O}\right)\left(\mathrm{NH}_{3}\right)_{4} \mathrm{~L}\right]^{2+}$ e

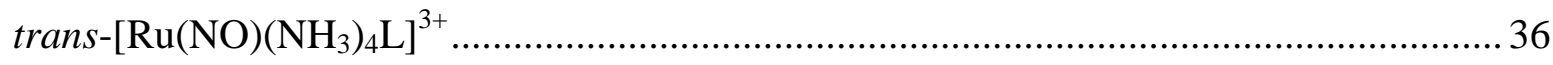

3.6 Avaliação da estabilidade dos novos íons complexos trans- $\left[\mathrm{Ru}(\mathrm{NO})\left(\mathrm{NH}_{3}\right)_{4} \mathrm{P}(\mathrm{O})\left(\mathrm{OCH}_{2} \mathrm{CH}_{3}\right)_{2}\right]^{2+}$ e trans- $\left[\mathrm{Ru}(\mathrm{CO})\left(\mathrm{NH}_{3}\right)_{4} \mathrm{P}(\mathrm{OH})_{3}\right]^{2+} \ldots \ldots \ldots \ldots \ldots \ldots . . . . . . . . .36$ 


\section{Sumário}

3.7 Determinação do $\mathrm{pK}_{\mathrm{a}}$ do éster de fosforo em trans- $\left[\mathrm{Ru}(\mathrm{CO})\left(\mathrm{NH}_{3}\right)_{4} \mathrm{P}(\mathrm{OH})_{3}\right]^{2+} \ldots \ldots \ldots \ldots . . . . .37$

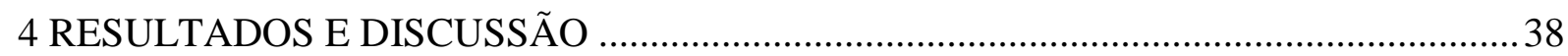

4.1 Dissociação do ligante de ácido fosforoso em trans- $\left[\mathrm{Ru}(\mathrm{NO})\left(\mathrm{NH}_{3}\right)_{4}\left(\mathrm{P}(\mathrm{OH})_{3}\right)\right]^{3+}$............. 39

4.1.1 Difração de Raio-X: trans- $\left[\mathrm{Ru}(\mathrm{NO})\left(\mathrm{NH}_{3}\right)_{4}\left(\mathrm{P}(\mathrm{O})(\mathrm{OH})_{2}\right)\right]\left(\mathrm{ZnCl}_{4}\right) \cdot 3 \mathrm{H}_{2} \mathrm{O} \ldots \ldots \ldots \ldots \ldots \ldots . . . . . . . . . .39$

4.1.2 Mecanismo de dissociação do ácido fosforoso em

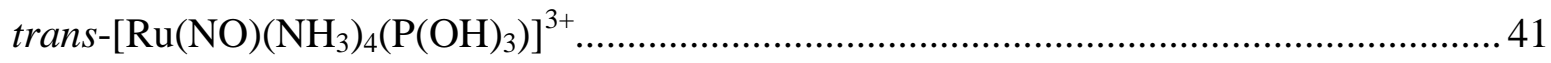

4.2 trans- $\left[\mathrm{Ru}(\mathrm{NO})\left(\mathrm{NH}_{3}\right)_{4}\left(\mathrm{P}(\mathrm{O})\left(\mathrm{OCH}_{2} \mathrm{CH}_{3}\right)_{2}\right)\right]\left(\mathrm{PF}_{6}\right)_{2}$ : síntese, caracterização e estabilidade em

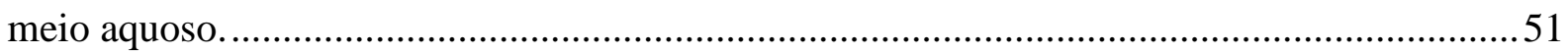

4.2.1 Caracterização do sólido trans- $\left[\mathrm{Ru}(\mathrm{NO})\left(\mathrm{NH}_{3}\right)_{4}\left(\mathrm{P}(\mathrm{O})\left(\mathrm{OCH}_{2} \mathrm{CH}_{3}\right)_{2}\right)\right]\left(\mathrm{PF}_{6}\right)_{2} \ldots \ldots \ldots \ldots . . . . . .51$

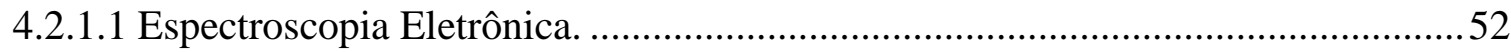

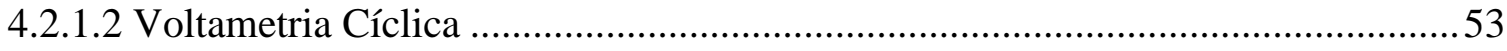

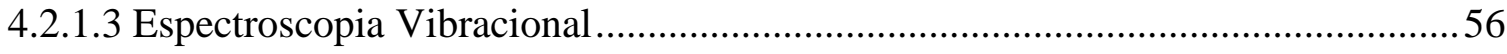

4.2.1.4 Ressonância Magnética Nuclear ........................................................................5

4.2.2 Estabilidade do íon trans- $\left[\mathrm{Ru}(\mathrm{NO})\left(\mathrm{NH}_{3}\right)_{4}\left(\mathrm{P}(\mathrm{O})\left(\mathrm{OCH}_{2} \mathrm{CH}_{3}\right)_{2}\right)\right]^{2+}$ em meio aquoso ....59

4.3 Estabilidade de ésteres de fósforo livres e coordenados a rutênio(II) em meio aquoso.....69

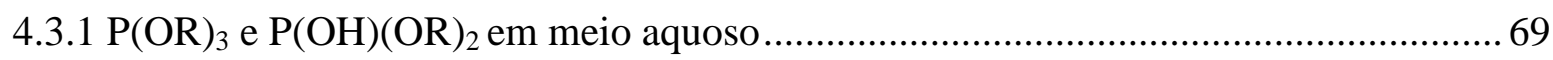

4.3.2 $\mathrm{P}(\mathrm{OR})_{3}$ e $\mathrm{P}(\mathrm{OH})(\mathrm{OR})_{2}$ coordenados aos fragmentos trans- $\left[\mathrm{Ru}\left(\mathrm{H}_{2} \mathrm{O}\right)\left(\mathrm{NH}_{3}\right)_{4}\right]^{2+} \mathrm{e}$

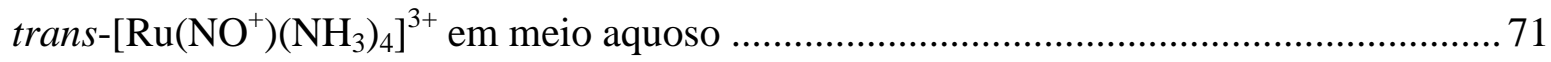

4.3.3 Propriedades eletroquímicas e $\mathrm{k}_{\text {-NO }}$ de nitrosilos complexos de fósforo(III)............... 79

4.4 Caracterização do íon complexo trans- $\left[\mathrm{Ru}(\mathrm{CO})\left(\mathrm{NH}_{3}\right)_{4}\left(\mathrm{P}(\mathrm{OH})_{3}\right)\right]^{2+}$ e sua estabilidade em

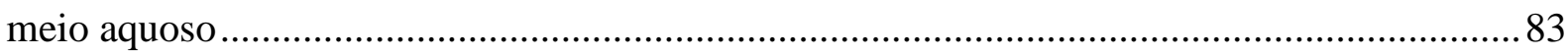

4.4.1 Caracterização do complexo trans- $\left[\mathrm{Ru}(\mathrm{CO})\left(\mathrm{NH}_{3}\right)_{4}\left(\mathrm{P}(\mathrm{O})(\mathrm{OH})_{2}\right)\right](\mathrm{X})$ em que

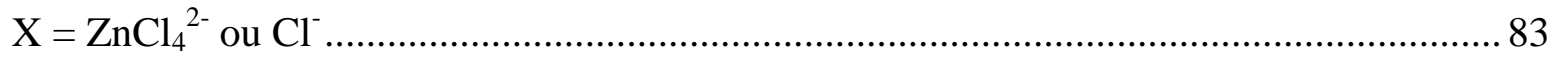

4.4.1.1 Difração de Raio-X: trans-[Ru(CO) $\left.\left(\mathrm{NH}_{3}\right)_{4}\left(\mathrm{P}(\mathrm{OH})_{3}\right)\right]\left(\mathrm{ZnCl}_{4}\right) \cdot 3 \mathrm{H}_{2} \mathrm{O}$................... 83

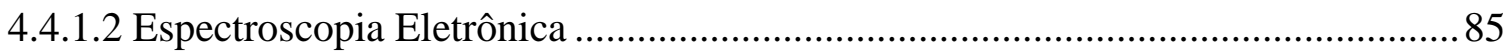

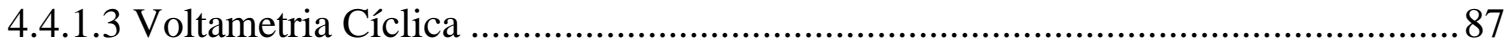

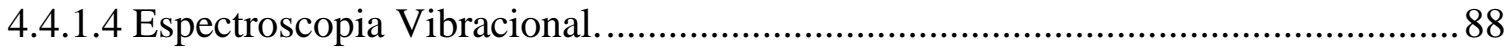

4.4.1.5 Ressonância Magnética Nuclear de ${ }^{31} \mathrm{P}$................................................................ 92

4.4.2 Estudo da estabilidade do complexo trans- $\left[\mathrm{Ru}(\mathrm{CO})\left(\mathrm{NH}_{3}\right)_{4}\left(\mathrm{P}(\mathrm{OH})_{3}\right)\right](\mathrm{Cl})_{3}$ em

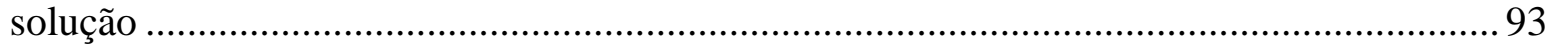

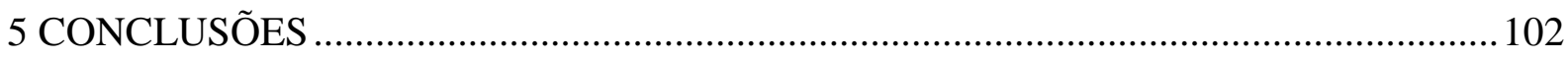

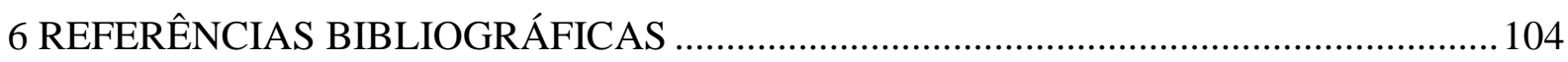




\section{INTRODUÇÃO}

\subsection{Moléculas sinalizadoras em meio biológico}

As moléculas de $\mathrm{NO}, \mathrm{CO}$ e $\mathrm{H}_{2} \mathrm{~S}$, antes conhecidas apenas por causarem preocupação devido a sua ação tóxica na indústria e no meio ambiente, nas últimas décadas tornaram-se alvo de crescente interesse por constituírem uma rede integrada de sinalizadores que regula diversos processos fisiológicos [1]. O termo "gasotransmitters" foi recentemente [2; 3] utilizado para referir-se a este grupo de moléculas. No entanto, tal termo é enganoso, pois estas moléculas são solúveis na concentração em que apresentam relevância biológica, não podendo, nestas situações, serem consideradas gases [1]. Estas moléculas apresentam características químicas em comum em meio biológico, sendo passíveis de coordenação a centros metálicos como o ferro em heme proteínas, e de participarem de processos de oxidoredução envolvendo tióis, como cisteína e glutationa, ou mesmo radicais livres tais como íon superóxido $\left(\mathrm{O}_{2}{ }^{-}\right)$e o radical hidroxila $\left(\mathrm{OH}^{*}\right)[1 ; 4 ; 5]$.

O estudo desta "biologia dos gases" teve início durante a década de 1980 quando Furchogott descobriu que as células endoteliais produziam uma determinada substância que ocasionava relaxação [6]. Posteriormente, Moncata [7] e Ignarro [8] provaram, de forma independente, que esta substância tratava-se do NO produzido endogenamente a partir da conversão da L-arginina em L-citrulina e NO [9; 10]. Desde então, inúmeros trabalhos tiveram o NO como tema central e, em 1992, a revista Science apresentou o NO como a molécula do ano. Ao longo dos anos, foi comprovado que o óxido nítrico apresenta uma variedade de funções fisiológicas, tais como o controle da pressão sanguínea [11], a neurotransmissão $[12 ; 13 ; 14]$, a resposta imunológica $[15 ; 16 ; 17]$, e a ação antioxidante [4; $18 ; 19]$. 


$$
\mathrm{NO}+\mathrm{e}^{-}+\mathrm{H}^{+} \longrightarrow \mathrm{HNO}
$$

O óxido nítrico pode ser reduzido por 1 elétron (reação 1) gerando íon nitroxila $\left(\mathrm{HNO}_{\mathrm{NO}}{ }^{-}\right)$em meio biológico. Esta molécula tem sido estudada com respeito a sua química e seus efeitos fisiológicos de forma comparativa ao NO [20; 21]. Apesar dos estudos com HNO serem recentes e ainda em número limitado, foi constatado o seu efeito em processos de vasorelaxação, sua ação protetora em enfartos do miocárdio e sua ação antioxidante $[1 ; 22$; 23; 24]. Diferentemente do NO, não há ainda comprovação da formação endógena do HNO como uma molécula sinalizadora, contudo há evidencias em reações bioquímicas relevantes que indicam a sua formação [22]. O NO apresenta tempo de meia vida em meio biológico que pode variar de 0,1 a $1 \mathrm{~s}$ [25], além de alta constante de difusão $\left(3,5 \times 10^{3} \mu \mathrm{m}^{2} \mathrm{~s}^{-1}\right)$ [26]. Por sua vez, o HNO possui tempo de meia vida de apenas 0,1 segundo, o que dificulta sua difusão, sendo este limitado ao local de sua formação [25].

Diferentemente do $\mathrm{NO}$ e do HNO, o monóxido de carbono (CO) apresenta-se relativamente inerte em meio biológico, mesmo em condições nas quais $\mathrm{NO}$ e HNO seriam facilmente degradados [1]. Por exemplo, o CO é menos sensível às condições celulares e à presença de oxidantes e redutores [1]. Apresentando efeitos biológicos similares ao NO [2], a interação entre $\mathrm{CO}$ e as metaloproteínas é a principal reação do $\mathrm{CO}$ em meio biológico, podendo até mesmo bloquear a ação das moléculas de $\mathrm{O}_{2}$ e $\mathrm{NO}$ [1]. A semelhança do $\mathrm{NO}$, o CO é uma molécula produzida endogenamente (a partir do catabolismo da heme oxigenase (HO) [2]) e o controle de sua concentração é crucial para se obter o efeito terapêutico desejado, isto em decorrência de sua toxicidade em altas concentrações. Para contornar este problema, o desenvolvimento de compostos exógenos capazes de liberar de forma eficiente, seletiva e controlada moléculas como $\mathrm{NO}, \mathrm{CO}$ e $\mathrm{H}_{2} \mathrm{~S}$ em meio biológico torna-se importante em intervenções terapêuticas. Os compostos transportadores de $\mathrm{NO}, \mathrm{CO}$ e $\mathrm{H}_{2} \mathrm{~S}$ 
podem ser orgânicos ou inorgânicos. No caso dos compostos inorgânicos, alguns são discriminados como "carregadores de fármacos", pois a molécula ativa em meio biológico normalmente encontra-se coordenada a um centro metálico sendo liberada após ativação térmica e/ou fotoquímica [27].

\subsection{Complexos metálicos como carregadores de fármacos}

Os complexos metálicos têm sido sistematicamente investigados quanto a sua atividade biológica e aplicação em doenças como câncer [28; 29; 30], deficiências cardiovasculares [31], artrite [32] e parasitoses [33], sendo que alguns destes complexos já se encontram disponíveis comercialmente. Dentre estes, destacam-se os compostos de platina descobertos na década de 1960 e utilizados no combate ao câncer, os quais, apesar de bastante empregados, apresentam problemas relacionados a efeitos colaterais e à resistência celular à droga [34]. Complexos de ferro também têm sido utilizados no tratamento de doenças cardiovasculares, anemia e câncer $[35 ; 36]$. Por exemplo, o $\mathrm{Na}_{2}\left[\mathrm{Fe}(\mathrm{NO})(\mathrm{CN})_{5}\right]$ é administrado clinicamente como vasodilatador, pois libera NO de forma rápida e eficiente in vivo [35; 36]. Entretanto, este é fotossensível e apresenta problemas de toxicidade associados à liberação de íons cianeto [31]. Outro centro metálico possível de ser utilizado como metalofarmacêutico é o ósmio, pois a composição de sua esfera de coordenação pode possibilitar a labilização da molécula de interesse com constante de velocidade próxima a dos processos celulares [37]. Todavia este metal apresenta alto custo, a síntese de seus compostos não é trivial e estes ainda apresentam elevada toxicidade [37].

Neste contexto, os complexos de rutênio apresentam perfil cinético similar ao da platina, mas sua toxicidade é consideravelmente menor [38], provavelmente devido a sua habilidade de mimetizar o ferro e ligar-se a biomoléculas. A atividade biológica de complexos 
de rutênio foi relatada pela primeira vez em 1950 e desde então tem merecido constante atenção [39; 40]. Como exemplo, o NAMI-A (trans-[Ru $\left.\left.{ }^{\mathrm{III}}(\mathrm{dmso}-S)(\mathrm{Im}) \mathrm{Cl}_{4}\right] \operatorname{ImH}\right)$ e o KP-1019 (trans-[Ru $\left.\left.{ }^{\text {III }}(\mathrm{Ind})_{2} \mathrm{Cl}_{4}\right] \mathrm{IndH}\right)$ são os complexos de $\mathrm{Ru}(\mathrm{III})$ mais promissores no tratamento do câncer $[41 ; 42]$.

As tetraaminas de rutênio(II) (Figura 1) constituem uma classe de compostos interessantes para aplicações em química medicinal, isto porque são solúveis em água, apresentam baixa citotoxicidade e facilidade de modulação [43; 44; 45]. As aminas em posição equatorial são inertes e assim as reações de substituição ocorrem apenas no eixo z, permitindo que esta classe de compostos seja um interessante modelo para o estudo da influência mútua entre os ligantes $\left(\mathrm{L}_{1} \mathrm{e} \mathrm{L}_{2}\right)$ e o centro metálico.

Figura 1 - Tetraaminas de rutênio.

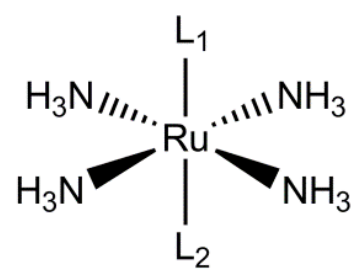

$\mathrm{NO}^{+}$e CO são isoeletrônicos $\left(\sigma_{\mathrm{s}}^{2}, \sigma_{\mathrm{s}}^{2}, \pi_{\mathrm{p}}^{4},{\sigma_{\mathrm{p}}}^{2}\right)$ e coordenam-se com facilidade às tetraaminas de rutênio(II) por meio da doação de um par de elétrons formando uma ligação $\sigma$ e uma ligação $\pi$ formada pela retrodoação de elétrons $4 \mathrm{~d} \pi$ do rutênio(II) para os orbitais p $\pi^{*}$ vazios destes ligantes. Sendo assim, ligantes insaturados coordenam-se ao rutênio(II) através de componentes $\sigma$ e $\pi$. O grau de retrodoação difere, sendo maior para o $\mathrm{NO}^{+}$do que para o $\mathrm{CO}$, devido à energia relativa dos orbitais $\mathrm{p} \pi^{*}[1]$.

Os nitrosilos complexos de rutênio foram extensivamente estudados. Observou-se que (Figura 1) ao se fixar o $\mathrm{L}_{1}$ como $\mathrm{NO}^{+}$, o ligante $\mathrm{L}_{2}$ torna-se capaz de modular o potencial de redução do ligante $\mathrm{NO}^{+}\left(\mathrm{E}_{\mathrm{NO}}^{+} / \mathrm{NO}{ }^{0}\right)$, a frequência de estiramento $\left(v_{\mathrm{NO}}^{+}\right)$, a constante de 
liberação de $\mathrm{NO}\left(\mathrm{k}_{-\mathrm{NO}}\right)$ e a reatividade química e fotoquímica do complexo como um todo [44; 45; 46; 47]. Esta capacidade de modulação está diretamente relacionada às características de $\mathrm{L}_{2}$ como seu grau de doação e recepção $\sigma$ e $\pi$, energia e simetria dos orbitais. Considerando-se que a ativação dos nitrosilos complexos para a doação de NO também pode ocorrer por via eletroquímica, quanto mais acessível for o $\mathrm{E}_{\mathrm{NO}}{ }^{+} \mathrm{NO}{ }^{0}$ aos redutores biológicos e quanto maior o valor numérico de k-NO, maior será a eficiência da doação de NO [46].

Neste aspecto, destaca-se o complexo trans-[Ru(NO)( $\left.\left.\mathrm{NH}_{3}\right)_{4} \mathrm{P}\left(\mathrm{OC}_{2} \mathrm{H}_{5}\right)_{3}\right]\left(\mathrm{PF}_{6}\right)_{3}$

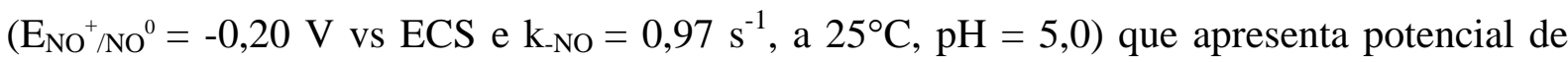
redução $\mathrm{NO}^{+} / \mathrm{NO}$ acessível a redutores biológicos tais como ascorbato, cisteína, glutationa, e NADH [48; 49; 50], além de rápida liberação do NO da esfera de coordenação do centro metálico. Tais características são conferidas pelo ligante de fósforo trivalente $\left(\mathrm{P}(\mathrm{OR})_{3}\right) \mathrm{em} \mathrm{L}_{2}$ que apresenta alto efeito e influência trans $[46 ; 51]$. Recentemente foi observado que além da redução por 1 elétron, o íon trans-[Ru(NO) $\left.\left(\mathrm{NH}_{3}\right)_{4} \mathrm{P}\left(\mathrm{OC}_{2} \mathrm{H}_{5}\right)_{3}\right]^{3+}$ também pode sofrer redução por 2 elétrons centrada no grupo nitrosônio $\left(\mathrm{E}_{\mathrm{NO} / \mathrm{NO}}=-0,70 \mathrm{~V}\right.$ vs $\left.\mathrm{ECS}\right)$, e assim liberar $\mathrm{HNO}$ em solução [52]. Dessa forma, este complexo poderia liberar seletivamente NO ou/e HNO em meio biológico em função do redutor químico, ou do potencial eletroquímico empregado [52].

Por outro lado, a química dos carbonilos de tetraaminas de rutênio ainda é pouco explorada com respeito a sua estabilidade química e sua capacidade de liberar $\mathrm{CO}$ em meio biológico [53]. Para o composto trans- $\left[\mathrm{Ru}(\mathrm{CO})\left(\mathrm{NH}_{3}\right)_{4} \mathrm{P}\left(\mathrm{OC}_{2} \mathrm{H}_{5}\right)_{3}\right]\left(\mathrm{PF}_{6}\right)_{2}$, foi observado que o monóxido de carbono é liberado em meio aquoso em uma ampla faixa de concentração hidrogeniônica e sem a necessidade de ativação fotoquímica ou eletroquímica (reação 2) [53]. Esta liberação espontânea do CO também se deve ao efeito trans labilizante do ligante trietil fosfito.

trans- $\left[\mathrm{Ru}(\mathrm{CO})\left(\mathrm{NH}_{3}\right)_{4}\left(\mathrm{P}\left(\mathrm{OC}_{2} \mathrm{H}_{5}\right)_{3}\right)\right]^{2+}+\mathrm{H}_{2} \mathrm{O} \underset{\mathrm{pH} ~ 3,0-12,0}{\stackrel{\mathrm{k} \sim 0^{-5} \mathrm{~s}^{-1}}{\longrightarrow}}$ trans- $\left[\mathrm{Ru}\left(\mathrm{H}_{2} \mathrm{O}\right)\left(\mathrm{NH}_{3}\right)_{4}\left(\mathrm{P}\left(\mathrm{OC}_{2} \mathrm{H}_{5}\right)_{3}\right)\right]^{2+}+\mathrm{CO}$ 


\subsection{Ligantes de fósforo(III): estabilidade, reatividade química e efeito e influência trans versus liberação de NO e CO}

Os fosfanos formam uma classe de compostos de fósforo(III) na qual estão inclusos os fosfitos $\left(\mathrm{P}(\mathrm{OR})_{3}\right)$ e as fosfinas $\left(\mathrm{P}(\mathrm{R})_{3}\right)$. Nestes compostos, o átomo de fósforo tem caráter bifílico, tendo em vista que apresenta um par de elétrons livre no orbital híbrido $\mathrm{sp}^{3}$ capaz de realizar uma ligação $\sigma$, e orbitais de simetria $3 \mathrm{~d} \pi$ vazios que possibilitam a retrodoação por meio de sua interação com os orbitais $4 \mathrm{~d} \pi$ preenchidos de centros metálicos como o rutênio(II). Dessa forma, os fosfanos agem como fortes recebedores $\pi$ e, de moderado a fortes, doadores $\sigma$. As fosfinas são bases $\sigma$ mais fortes e ácidos $\pi$ mais fracos que os correspondentes fosfitos e apresentam efeito trans superior ao dos fosfitos, mas influência trans inferior [46].

A característica bifílica dos fosfanos pode ser modulada em função dos grupos substituintes R. A seguinte ordem de acidez $\pi$ para fosfitos foi proposta com base em dados eletroquímicos para o sistema trans- $\left[\mathrm{Ru}\left(\mathrm{H}_{2} \mathrm{O}\right)\left(\mathrm{NH}_{3}\right)_{4} \mathrm{P}\left(\mathrm{OR}_{3}\right)_{3}\right]^{2+}: \mathrm{P}(\mathrm{OPh})_{3}>\mathrm{P}\left(\mathrm{OC}_{2} \mathrm{H}_{4} \mathrm{Cl}\right)_{3}>$ $\mathrm{P}\left(\mathrm{OCH}_{3}\right)_{3}>\mathrm{P}\left(\mathrm{OC}_{2} \mathrm{H}_{5}\right)_{3}>\mathrm{P}\left(\mathrm{O}^{\mathrm{i}} \mathrm{C}_{3} \mathrm{H}_{7}\right)_{3}>\mathrm{P}\left(\mathrm{OC}_{4} \mathrm{H}_{9}\right)_{3}>\mathrm{P}(\mathrm{OH})_{3}>\mathrm{P}(\mathrm{OH})\left(\mathrm{OC}_{2} \mathrm{H}_{5}\right)_{2}>\mathrm{P}(\mathrm{O})(\mathrm{OH})_{2}$ $>\mathrm{P}(\mathrm{O})\left(\mathrm{OC}_{2} \mathrm{H}_{5}\right)_{2}[46 ; 54]$.

Os fosfanos também são conhecidos por apresentarem elevado efeito e influência trans, ou seja, por labilizarem (efeito cinético) e enfraquecerem a ligação (efeito termodinâmico), respectivamente, do ligante em posição trans. Assim, no sistema de tetraaminas de rutênio (Figura 1), quando fixamos $\mathrm{L}_{1}=\mathrm{NO}^{+} / \mathrm{CO}$ e $\mathrm{L}_{2}=$ fosfano, podemos modular as propriedades termodinâmicas destas moléculas de interesse biológico e fazer com que as mesmas possam ser labilizadas com constantes de velocidade de interesse, de acordo com o alvo biológico. 
Dentre os fosfitos, há os trialquil fosfitos $\left(\mathrm{P}(\mathrm{OR})_{3}\right)$ que apresentam uma única geometria (piramidal), e os fosfitos do tipo $\mathrm{P}(\mathrm{OH})(\mathrm{OR})_{2}$, como os dialquil e monoalquil fosfitos e o ácido fosforoso, que podem apresentar as geometrias piramidal $\left(\mathrm{P}(\mathrm{OH})(\mathrm{OR})_{2}\right)$ e tetraédrica $\left(\mathrm{P}(\mathrm{O})(\mathrm{H})(\mathrm{OR})_{2}\right)$, reação 3. Estas duas formas geométricas participam de um equilíbrio tautomérico em que a constante de equilíbrio $(\mathrm{K})$ é da ordem de $10^{7}$ a $10^{10}$ a favor da forma tetraédrica [55]. Apesar disto, a coordenação a centros metálicos é capaz de estabilizar a forma piramidal dos $\mathrm{P}(\mathrm{OH})(\mathrm{OR})_{2}$ por meio da retrodoação $\left(4 \mathrm{~d} \pi\left(\mathrm{Ru}^{\mathrm{II}}\right) \rightarrow 3 \mathrm{~d} \pi(\mathrm{P})\right)$, sugerindo que a ligação $\pi \mathrm{Ru}(\mathrm{II})-\mathrm{P}(\mathrm{III})$ é mais forte que a ligação $\pi \mathrm{O}-\mathrm{P}(\mathrm{III})[56 ; 57 ; 58]$. As espécies $\mathrm{P}(\mathrm{OH})(\mathrm{OR})_{2}$ também apresentam um próton dissociável, o qual pode ter sua acidez alterada com a coordenação [56; 59] e desta forma modificando suas características como ligante.

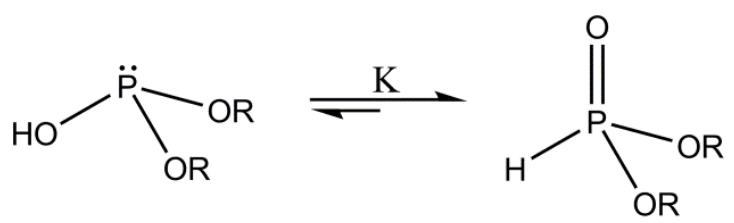

Os fosfitos são conhecidos por sua alta reatividade, uma vez que em solução são facilmente hidrolisados e oxidados [60]. Devido ao fato das reações de hidrólise ocorrerem muito rapidamente em meio aquoso, o mecanismo por meio do qual tais reações ocorrem não é totalmente conhecido e é foco de estudos devido à relevância de sua química e às possibilidades de aplicação dos fósforos na química de ácidos nucleicos e em processos industriais [60].

A hidrólise de ésteres de fósforos pode ocorrer por meio de diferentes mecanismos (Figura 2) [61]. A Figura 2a mostra a hidrólise via mecanismo de Michaelis-Arbusov [62], no qual ocorre inicialmente um ataque eletrofílico do hidrogênio da água sobre o átomo de fósforo, seguido de um ataque nucleofílico do oxigênio de uma segunda molécula de água 
sobre o carbono- $\alpha$ do fosfito e, consequente quebra da ligação O-C. Entretanto, Aksnes [63; 64] questionou este mecanismo com base em resultados de espectroscopia de infravermelho, nos quais foram analisadas as frequências de estiramento $\mathrm{P}=\mathrm{O}\left(\mathrm{P}={ }^{16} \mathrm{O}\right.$ em $1258 \mathrm{~cm}^{-1}$ e $\mathrm{P}={ }^{18} \mathrm{O}$ em $\left.1216 \mathrm{~cm}^{-1}\right)$ do produto da hidrólise utilizando oxigênio marcado $\left({ }^{18} \mathrm{O}\right)$. Tais experimentos indicaram que a reação de hidrólise em fosfitos se daria preferencialmente de forma similar a de ésteres orgânicos, ou seja, por meio do ataque nucleofílico do oxigênio da água no átomo de fósforo e consequente quebra da ligação P-OR (Figura 2b).

Figura 2 - Mecanismos de reação propostos para a hidrólise de fosfitos (a) mecanismo de Michaelis-Arbusov, (b) mecanismo proposto por Aksnes (similar a ésteres orgânicos) (c) catálise ácida via mecanismo de Michaelis-Arbusov e (d) catálise ácida via mecanismo proposto por Aksnes. $\mathrm{O}^{*}={ }^{18 / 17} \mathrm{O}$.

(a) $: \mathrm{P}(\mathrm{OR})_{3}+2 \mathrm{H}_{2} \mathrm{O} * \longrightarrow$

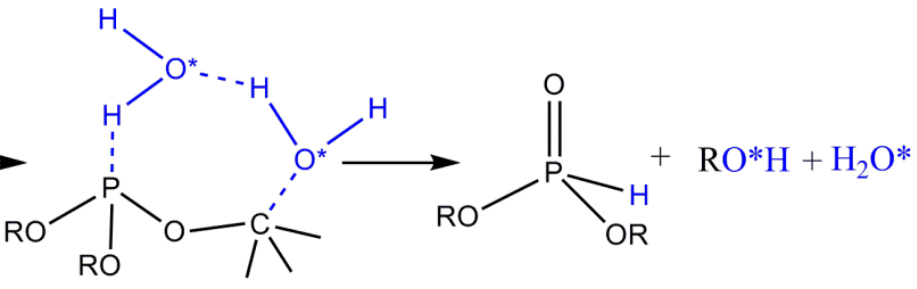

(b)

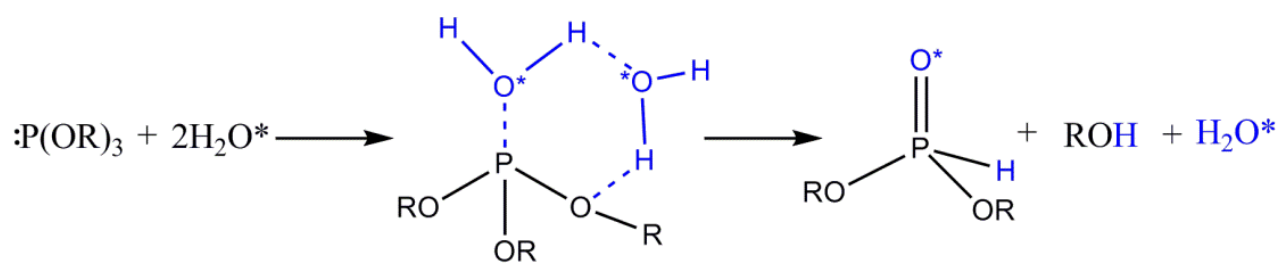

(c)

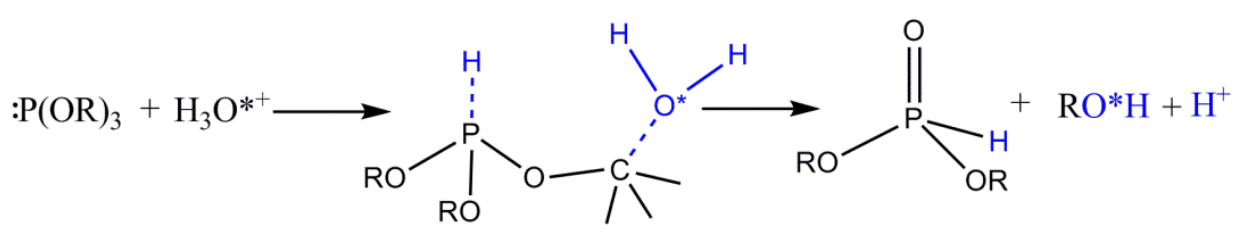

(d)

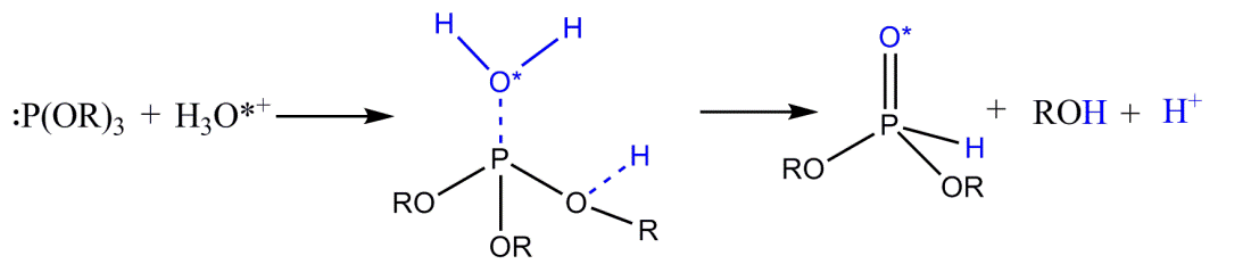

A hidrólise em fosfitos também pode ocorrer via catálise ácida (Figura 2 c e d). Segundo Michaelis-Arbusov [62] inicialmente ocorreria a protonação do par de elétrons livre 
do fosfito, seguida do pelo ataque nucleofílico no carbono- $\alpha$ do fosfito e, consequente quebra da ligação O-C. Entretanto, Aksnes [63] sugere a protonação do átomo de oxigênio, seguido pelo ataque nucleofílico sobre o átomo de fósforo.

Foram realizados experimentos utilizando ${ }^{17} \mathrm{O}$ e ${ }^{31} \mathrm{P}$ RMN com água marcada com ${ }^{17} \mathrm{O}$ e ${ }^{18} \mathrm{O}$, respectivamente $[61 ; 65]$. A análise dos produtos da hidrólise em solvente orgânico com frações controladas de água (sem controle de $\mathrm{pH}$ ), demonstraram formação do fosfito hidrolisado contendo o oxigênio marcado em sua estrutura, ou seja, a quebra da ligação P-OR em detrimento da quebra da ligação O-C (Figura 2b) [61; 65].

Apesar da alta reatividade dos fosfitos em meio aquoso, a existência de diversos complexos contendo moléculas de fosfitos coordenadas, como por exemplo: trans- $\left[\mathrm{Ru}\left(\mathrm{H}_{2} \mathrm{O}\right)\left(\mathrm{NH}_{3}\right)_{4} \mathrm{P}(\mathrm{OR})_{3}\right]\left(\mathrm{PF}_{6}\right)_{2}$ em que $\mathrm{P}(\mathrm{OR})_{3}=\mathrm{P}\left(\mathrm{OCH}_{3}\right)_{3}, \mathrm{P}\left(\mathrm{OC}_{2} \mathrm{H}_{5}\right), \mathrm{P}\left(\mathrm{O}^{\mathrm{i}} \mathrm{C}_{3} \mathrm{H}_{7}\right)_{3}$ e $\mathrm{P}\left(\mathrm{OC}_{4} \mathrm{H}_{9}\right)_{3}[66 ; 67]$; indica que a coordenação a centros metálicos, como o rutênio(II), pode vir a estabilizar tais moléculas, de forma que as reações de oxidação e hidrólise podem ter suas constantes de velocidade alteradas.

De fato, enquanto os $\mathrm{P}(\mathrm{OR})_{3}$ podem ser facilmente oxidados por $\mathrm{I}_{2}, \mathrm{Ce}^{4+}$ e $\mathrm{H}_{2} \mathrm{O}_{2}$ [60], quando estas moléculas estão coordenadas ao centro de $\mathrm{Ru}(\mathrm{II})$, como em trans- $\left[\mathrm{Ru}\left(\mathrm{H}_{2} \mathrm{O}\right)\left(\mathrm{NH}_{3}\right)_{4} \mathrm{P}(\mathrm{OR})_{3}\right]\left(\mathrm{PF}_{6}\right)_{2}$ e trans- $\left[\mathrm{Ru}\left(\mathrm{NH}_{3}\right)_{4}\left(\mathrm{P}(\mathrm{OR})_{3}\right)_{2}\right]\left(\mathrm{PF}_{6}\right)_{2}$, a adição de $\mathrm{Br}_{2}$, $\mathrm{Ce}^{4+}$ e $\mathrm{H}_{2} \mathrm{O}_{2}$ não leva a oxidação do fosfito [67; 68].

A hidrólise do trietil fosfito promovida pela umidade do ar no complexo trans-[Ru(NO)(NH$\left.)_{4} \mathrm{P}\left(\mathrm{OC}_{2} \mathrm{H}_{5}\right)_{3}\right]\left(\mathrm{PF}_{6}\right)_{3}$ em estado sólido foi acompanhada ao longo de 8 meses observando como produtos trans-[Ru(NO) $\left.\left(\mathrm{NH}_{3}\right)_{4} \mathrm{P}(\mathrm{OH})(\mathrm{OEt})_{2}\right]\left(\mathrm{PF}_{6}\right)_{3}$ e etanol [69]. Comportamento similar foi descrito para o $\left[\mathrm{Mo}(\mathrm{CO})_{5} \mathrm{P}(\mathrm{OH})(\mathrm{OEt})_{2}\right]$, o qual é convertido em estado sólido a $\left[\mathrm{Mo}(\mathrm{CO})_{5}\left(\mathrm{P}(\mathrm{OH})_{3}\right)\right][70]$. Entretanto, apesar da hidrólise de fosfito também ter sido evidenciada quando estes estão coordenados a centros metálicos, nenhum dado cinético ou mesmo elucidação do possível mecanismo foi reportado. 


\section{OBJETIVO}

A coordenação de fosfanos $\left(\mathrm{P}(\mathrm{OR})_{3}\right.$ e $\left.\left(\mathrm{PR}_{3}\right)\right)$ a centros metálicos induz mudanças substanciais nas características do centro metálico e nas propriedades desses ligantes em si. Isto permite que complexos metálicos sejam modelos para estudos das alterações nas reatividades, dos ligantes e dos centros metálicos, induzidas pela coordenação. Este trabalho tem por objetivo estudar a estabilidade dos ésteres de fósforo livres e coordenados a centros de rutênio(II). Assim como adquirir conhecimento a respeito do mútuo efeito e influência trans que existe entre os ésteres de fósforo(III) e os ligantes nitrosônio $\left(\mathrm{NO}^{+}\right)$e monóxido de carbono (CO) em complexos metálicos utilizando como modelo as tetraaminas de rutênio(II). 


\section{METODOLOGIA}

\subsection{Reagentes}

Todos os reagentes utilizados eram de grau analítico e foram utilizados como fornecidos. Os solventes em geral foram purificados por meio de destilação seguindo procedimentos descritos na literatura [71].

Os ésteres de fósforo foram previamente caracterizados por meio de Ressonância Magnética Nuclear (RMN) de próton $\left({ }^{1} \mathrm{H}\right)$ e Espectroscopia Vibracional (IV) para verificar a presença de fósforo oxidado e/ou hidrolisado. Em caso de necessidade de purificação, o éster de fósforo foi destilado em pressão reduzida e sob fluxo de argônio, conforme descrito na literatura [72]. O armazenamento dos trialquil e dialquil fosfitos foi realizado em ambiente fechado na presença de sílica e do ácido fosforoso em dessecador a vácuo.

As manipulações foram sempre conduzidas em atmosfera de argônio em sistemas fechados e as transferências realizadas via cânulas de teflon [73]. O argônio utilizado foi previamente tratado para retirada de traços de oxigênio e posterior secagem sendo conduzido por dois frascos lavadores com solução ácida de cromo(II) e, em seguida, por um frasco contendo ácido sulfúrico.

\subsection{Sínteses}

\subsubsection{Síntese do complexo $\left[\mathrm{Ru}\left(\mathrm{NH}_{3}\right)_{5} \mathrm{Cl}\right](\mathrm{Cl})_{2}[74]$}

2,00 g de $\mathrm{RuCl}_{3 \times} \mathrm{H}_{2} \mathrm{O}$ foram dissolvidos em $27 \mathrm{~mL}$ de água destilada. Em seguida, adicionou-se cuidadosamente $26 \mathrm{~mL}$ de monohidrato de hidrazina $\left(\mathrm{NH}_{2} \mathrm{NH}_{2} \cdot \mathrm{H}_{2} \mathrm{O}\right)$ sob 
agitação constante e banho de gelo. A mistura permaneceu reagindo por um período de 6 horas. A seguir, adicionou-se $50 \mathrm{~mL}$ de ácido clorídrico $(\mathrm{HCl})$ concentrado sob banho de gelo. A solução foi refluxada por 2 horas, ocorrendo a formação de precipitado amarelo. Após o resfriamento, o sólido foi coletado por filtração, em filtro de porosidade média, e lavado com solução gelada de $\mathrm{HCl} 1,5 \mathrm{~mol} \mathrm{~L}^{-1}$.

Recristalização: O sólido obtido foi adicionado a $200 \mathrm{~mL}$ de $\mathrm{HCl} 0,1 \mathrm{~mol} \mathrm{~L}^{-1}$ e aquecido (50$60^{\circ} \mathrm{C}$ ) até dissolução completa. Procedeu-se, então, filtração a quente da solução em funil de placa porosa. Ao filtrado, de coloração amarelo claro, foi adicionado excesso de $\mathrm{HCl}$ concentrado (cerca de $50 \mathrm{~mL}$ ) sob banho de gelo. O sólido obtido foi filtrado e seco a vácuo e estocado ao abrigo de luz. Rendimento: $55 \%$.

\subsubsection{Síntese do complexo $\left[\mathrm{Ru}\left(\mathrm{H}_{2} \mathrm{O}\right)\left(\mathrm{NH}_{3}\right)_{5}\right]\left(\mathrm{PF}_{6}\right)_{2}[75]$}

O íon aquapentaaminrutênio(II) foi preparado por meio da adição de $100 \mathrm{mg}$ de $\left[\mathrm{Ru}\left(\mathrm{NH}_{3}\right)_{5} \mathrm{Cl}\right](\mathrm{Cl})_{2}$ a $4 \mathrm{~mL}$ de ácido trifluoroacético $\left(\mathrm{CF}_{3} \mathrm{COOH}\right) 0,10 \mathrm{~mol} \mathrm{~L}^{-1}$ previamente desaerados. Em seguida, pastilhas de amalgama de zinco foram adicionadas. A redução dos íons $\mathrm{Ru}(\mathrm{III})$ a $\mathrm{Ru}(\mathrm{II})$ pode ser observada pela dissolução do complexo de partida e pela mudança da cor da solução de amarelo claro para amarelo intenso.

Quando se desejou isolar este complexo, $0,5 \mathrm{~g}$ de $\mathrm{NH}_{4} \mathrm{PF}_{6}$ foram adicionados. $\mathrm{O}$ precipitado, então, foi filtrado em glove bag, lavado com etanol previamente desaerado e seco em dessecador a vácuo por 20 minutos. Este composto é sensível à oxidação por oxigênio, portanto é sempre utilizado imediatamente após sua preparação. Rendimento: $83 \%$. 


\subsubsection{Síntese do complexo trans- $\left[\mathrm{Ru}\left(\mathrm{NH}_{3}\right)_{4}\left(\mathrm{P}(\mathrm{OR})_{3}\right)_{2}\right]\left(\mathrm{PF}_{6}\right)_{2}[66]$}

$200 \mathrm{mg}$ de $\left[\mathrm{Ru}\left(\mathrm{H}_{2} \mathrm{O}\right)\left(\mathrm{NH}_{3}\right)_{5}\right]\left(\mathrm{PF}_{6}\right)_{2}$ recentemente preparados foram adicionados a 10 $\mathrm{mL}$ de acetona desaerada. Em seguida, acrescentou-se $1 \mathrm{~mL}$ do trialquil fosfito desejado $\left(\mathrm{P}(\mathrm{OR})_{3}=\mathrm{P}\left(\mathrm{OCH}_{3}\right)_{3}, \mathrm{P}\left(\mathrm{OC}_{2} \mathrm{H}_{5}\right), \mathrm{P}\left(\mathrm{O}^{\mathrm{i}} \mathrm{C}_{3} \mathrm{H}_{7}\right)_{3}\right.$ e $\left.\mathrm{P}\left(\mathrm{OC}_{4} \mathrm{H}_{9}\right)_{3}\right)$ e a mistura foi deixada reagir por 1 hora sob fluxo de argônio. O excesso de acetona foi evaporado e o trans-[Ru(NH$\left.)_{4}\left(\mathrm{P}(\mathrm{OR})_{3}\right)_{2}\right]\left(\mathrm{PF}_{6}\right)_{2}$ foi precipitado pela adição de éter:etanol na proporção 2:1. O sólido amarelo foi filtrado e lavado com a mistura éter:etanol, seco e estocado a vácuo e ao abrigo de luz. Rendimento: 65-80\%.

\subsubsection{Síntese dos complexos trans- $\left[\mathrm{Ru}\left(\mathrm{H}_{2} \mathrm{O}\right)\left(\mathrm{NH}_{3}\right)_{4} \mathrm{P}(\mathrm{OR})_{3}\right]\left(\mathrm{PF}_{6}\right)_{2} \quad$ e trans- $\left[\mathrm{Ru}(\mathrm{NO})\left(\mathrm{NH}_{3}\right)_{4} \mathbf{P}(\mathrm{OR})_{3}\right]\left(\mathrm{PF}_{6}\right)_{3}, \quad \mathbf{P}(\mathrm{OR})_{3}=\mathbf{P}\left(\mathrm{OCH}_{3}\right)_{3}, \quad \mathbf{P}\left(\mathrm{OC}_{2} \mathrm{H}_{5}\right), \quad \mathbf{P}\left(\mathrm{O}^{\mathrm{i}} \mathrm{C}_{3} \mathrm{H}_{7}\right)_{3}$ $\mathrm{P}\left(\mathrm{OC}_{4} \mathrm{H}_{9}\right)_{3}[51 ; 76]$}

$200 \mathrm{mg}$ de trans- $\left[\mathrm{Ru}\left(\mathrm{NH}_{3}\right)_{4}\left(\mathrm{P}(\mathrm{OR})_{3}\right)_{2}\right]\left(\mathrm{PF}_{6}\right)_{2}$ foram adicionados a $200 \mathrm{~mL}$ de solução $\mathrm{CF}_{3} \mathrm{COOH} \quad 0,001 \mathrm{~mol} \mathrm{~L}^{-1}$ previamente desaerados. Nos casos em que o complexo não solubilizou neste meio $\left(\mathrm{P}(\mathrm{OR})_{3}=\mathrm{P}\left(\mathrm{O}^{\mathrm{i}} \mathrm{C}_{3} \mathrm{H}_{7}\right)_{3}\right.$ e $\left.\mathrm{P}\left(\mathrm{OC}_{4} \mathrm{H}_{9}\right)_{3}\right)$, foi adicionado etanol desaerado até solubilização. A reação foi mantida a $40{ }^{\circ} \mathrm{C}$ e sob fluxo de argônio por 6 horas, sendo acompanhada a formação da banda em $316 \mathrm{~nm}$ por Espectroscopia Eletrônica (UV-vis). Em seguida, o solvente foi evaporado em evaporador rotativo até volume próximo a $3 \mathrm{~mL}$.

Quando se objetivou obter o sólido trans- $\left[\mathrm{Ru}\left(\mathrm{H}_{2} \mathrm{O}\right)\left(\mathrm{NH}_{3}\right)_{4} \mathrm{P}(\mathrm{OR})_{3}\right]\left(\mathrm{PF}_{6}\right)_{2}$ acrescentouse éter:etanol (2:1) obtendo-se um sólido amarelo claro, o qual foi coletado por filtração, seco e armazenado a vácuo e ao abrigo de luz.

Para a síntese do sólido trans-[Ru(NO)(NH$\left.)_{4} \mathrm{P}(\mathrm{OR})_{3}\right]\left(\mathrm{PF}_{6}\right)_{3}$, a solução de $3 \mathrm{~mL}$ foi colocada sob fluxo de argônio e adicionou-se $2 \mathrm{~mL}$ de uma solução 2,0 mol L ${ }^{-1}$ de $\mathrm{CF}_{3} \mathrm{COOH}$ 
previamente desaerada. A essa mistura foram adicionados $200 \mathrm{mg}$ de $\mathrm{NaNO}_{2}$ e $300 \mathrm{mg}$ de $\mathrm{NH}_{4} \mathrm{PF}_{6}$. Após a refrigeração da solução houve formação de um sólido cristalino rosa, o qual foi coletado por filtração, lavado com éter etílico, seco e estocado a vácuo ao abrigo de luz. Rendimento: $30-50 \%$.

Todos os compostos sintetizados foram sempre identificados por espectroscopia eletrônica e de infravermelho, RMN de ${ }^{31} \mathrm{P},{ }^{1} \mathrm{H}$ e ${ }^{13} \mathrm{C}$ e voltametria cíclica, sendo os dados obtidos comparados aos descritos na literatura.

\subsection{Sínteses dos novos complexos}

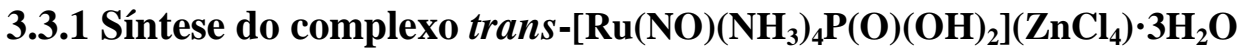

Neste caso, o íon $\left[\mathrm{Ru}\left(\mathrm{H}_{2} \mathrm{O}\right)\left(\mathrm{NH}_{3}\right)_{5}\right]^{2+}$ não foi isolado previamente ao seu uso. Sendo assim, após a redução por amalgama de zinco de $100 \mathrm{mg}$ do complexo $\left[\mathrm{Ru}\left(\mathrm{NH}_{3}\right)_{5}(\mathrm{Cl})\right] \mathrm{Cl}_{2} \mathrm{em}$ $4 \mathrm{~mL}$ de solução $0,10 \mathrm{~mol} \mathrm{~L}^{-1} \mathrm{CF}_{3} \mathrm{COOH}$ (item 3.2.2) foram acrescentados $280 \mathrm{mg}$ de ácido fosforoso (excesso molar de 10 vezes o número de mols de rutênio). Após uma hora de reação iniciou-se o borbulhamento de $\mathrm{NO}_{(\mathrm{g})}$, o qual foi mantido por 5 horas obtendo-se então uma solução vermelho intenso. Em seguida, acrescentou-se $0,5 \mathrm{~mL}$ de $\mathrm{HCl}$ 6,0 mol L ${ }^{-1}$ e a solução teve seu volume reduzido a 1-2 mL por evaporação. Após refrigeração da solução por cerca de 12 horas, um sólido vermelho cristalino foi obtido e então filtrado, lavado com acetona, seco e estocado em dessecador a vácuo na ausência de luz. Rendimento: 80\% 


\subsubsection{Síntese do complexo trans- $\left[\mathrm{Ru}(\mathrm{CO})\left(\mathrm{NH}_{3}\right)_{4} \mathrm{P}(\mathrm{O})(\mathrm{OH})_{2}\right](\mathrm{X})$ em que $\mathrm{X}=\mathrm{ZnCl}_{4}{ }^{2-}$} ou $\mathrm{Cl}^{-}$

Esta síntese foi realizada segundo procedimento descrito no item 3.3.1, mas utilizando-se o $\mathrm{CO}(\mathrm{g})$ para borbulhamento. Após 5 horas de reação a solução apresentou coloração amarelo claro. Após refrigeração, observou-se a formação de agulhas de um sólido incolor de aparência cristalina. Quando analisado por Raio-X foi identificado o complexo trans- $\left[\mathrm{Ru}(\mathrm{CO})\left(\mathrm{NH}_{3}\right)_{4} \mathrm{P}(\mathrm{O})(\mathrm{OH})_{2}\right]\left(\mathrm{ZnCl}_{4}\right)$. Ao acrescentar cerca de $30 \mathrm{~mL}$ de éter:etanol $(3: 1)$ à solução ocorreu a formação de um sólido branco, o qual foi filtrado, seco e estocado em dessecador a vácuo na ausência de luz. Este último corresponde ao complexo supracitado contendo o íon $\mathrm{Cl}^{-}$como contra íons (trans- $\left.\left[\mathrm{Ru}(\mathrm{CO})\left(\mathrm{NH}_{3}\right)_{4} \mathrm{P}(\mathrm{O})(\mathrm{OH})_{2}\right] \mathrm{Cl}\right)$. Rendimento: $82 \%$

\subsubsection{Síntese do complexo trans $-\left[\mathrm{Ru}\left(\mathrm{H}_{2} \mathrm{O}\right)\left(\mathrm{NH}_{3}\right)_{4} \mathrm{P}(\mathrm{OH})\left(\mathrm{OCH}_{2} \mathrm{CH}_{3}\right)_{2}\right]\left(\mathrm{PF}_{6}\right)_{2}$}

$3,4 \times 10^{-4}$ mols de trans- $\left[\mathrm{Ru}\left(\mathrm{H}_{2} \mathrm{O}\right)\left(\mathrm{NH}_{3}\right)_{5}\right]\left(\mathrm{PF}_{6}\right)_{2}(169 \mathrm{mg})$ e $3,9 \times 10^{-4}$ mols de dietil fosfito $(0,5 \mathrm{~mL})$ foram adicionados em $15 \mathrm{~mL}$ de acetona desaerada e mantidos em atmosfera de argônio por 1 hora. Esta solução foi evaporada até volume de 1-2 mL e, em seguida, acrescentou-se $20 \mathrm{~mL}$ de éter:etanol (10:1) obtendo-se um óleo amarelo, o qual foi seco a vácuo. Inúmeras tentativas foram realizadas no sentido de obter um sólido, porém não se obteve sucesso. 


\subsubsection{Síntese do complexo trans- $\left[\mathrm{Ru}(\mathrm{NO})\left(\mathrm{NH}_{3}\right)_{4} \mathrm{P}(\mathrm{O})\left(\mathrm{OCH}_{2} \mathrm{CH}_{3}\right)_{2}\right]\left(\mathrm{PF}_{6}\right)_{2}$}

$3,4 \times 10^{-4}$ mols de trans- $\left[\mathrm{Ru}\left(\mathrm{H}_{2} \mathrm{O}\right)\left(\mathrm{NH}_{3}\right)_{5}\right]\left(\mathrm{PF}_{6}\right)_{2}(169 \mathrm{mg})$ e $3,9 \times 10^{-4}$ mols de dietil fosfito $(0,5 \mathrm{~mL})$ foram adicionados em $15 \mathrm{~mL}$ de acetona sob atmosfera de argônio e deixados reagir por 1 hora. Em seguida, borbulhou-se $\mathrm{NO}_{(\mathrm{g})}$ nesta solução por 4 horas. A acetona foi então evaporada e adicionou-se pela ordem $100 \mathrm{mg}$ de $\mathrm{NH}_{4} \mathrm{PF}_{6}$ e $50 \mathrm{~mL}$ de éter:etanol (10:1). O sólido obtido foi filtrado, seco e estocado a vácuo e na ausência de luz. Rendimento: 34\%.

\subsection{Caracterização}

\subsubsection{Análise Elementar}

As Análises Elementar de carbono, nitrogênio e hidrogênio foram realizadas no equipamento da Perkin-Elmer CNH 2400 alocado no Laboratório de Análise Elementar do Instituto de Química da USP, São Paulo.

\subsubsection{Espectroscopia Eletrônica}

Os espectros eletrônicos foram obtidos em um espectrofotômetro Hitachi, modelo U3501, utilizando celas de quartzo (caminho óptico de 1,0 cm ou 5,0 cm). As análises foram conduzidas em meio aquoso com força iônica e concentração hidrogeniônicas préestabelecidas, sendo a temperatura controlada por banho termostático. As soluções dos complexos sempre foram previamente desaeradas e transferidas em atmosfera inerte. 


\subsubsection{Espectroscopia Vibracional}

Os espectros na região do infravermelho foram obtidos em espectrofotômetro BOMEM FTIR-102. Em estado sólido, os espectros dos compostos foram realizados em $\mathrm{KBr}$ na região de 400 a $4000 \mathrm{~cm}^{-1}$ com resolução de $\pm 2 \mathrm{~cm}^{-1}$. Os espectros em solução foram conduzidos em janela de silício, com espaçador de teflon de $0,10 \mathrm{~mm}$, concentração de complexo de $5,0 \times 10^{-2} \mathrm{~mol} \mathrm{~L}^{-1}$ e região de 2100 a $1750 \mathrm{~cm}^{-1}$ com resolução de $\pm 2 \mathrm{~cm}^{-1}$.

\subsubsection{Experimentos eletroquímicos (VC e VDP)}

As medidas eletroquímicas foram efetuadas em um potenciostato da PAR (Princeton Applied Research), modelo 264A, utilizando célula eletroquímica constituída de um sistema de três eletrodos: um eletrodo de trabalho de carbono vítreo, um contra-eletrodo de platina e um eletrodo de referência de calomelano saturado (ECS). Os experimentos foram conduzidos a temperatura controlada por banho termostático, em eletrólito de força iônica e $\mathrm{pH}$ controlados.

\subsubsection{Calculo de $\mathbf{k}_{\text {-NO }}$ via eletroquímica: método de Nicholson e Shain [77]}

A redução do ligante nitrosônio nos íons trans- $\left[\mathrm{Ru}(\mathrm{NO})\left(\mathrm{NH}_{3}\right)_{4} \mathrm{~L}\right]^{3+}$ e a consequente liberação de óxido nítrico trata-se de uma transferência de carga seguida de uma reação química irreversível (reações 4 e 5). Quando $\mathrm{k}_{\mathrm{f}}$ é muito pequeno, ou seja, quando a reação química é desprezível, o processo eletroquímico (reação 4) observado é semelhante a um processo reversível. Entretanto, quando o valor de $\mathrm{k}_{\mathrm{f}}$ é suficientemente alto, o processo eletroquímico observado possui aparência de processo irreversível, de forma que a corrente de 
pico anódico $\left(\mathrm{i}_{\mathrm{pa}}\right)$ e a corrente de pico catódico $\left(\mathrm{i}_{\mathrm{pc}}\right)$ passam a relacionar-se com o parâmetro cinético.

$$
\begin{aligned}
& \text { trans } \left.\left.-\left[\mathrm{Ru}(\mathrm{NO})\left(\mathrm{NH}_{3}\right)_{4} \mathrm{~L}\right)\right]^{3+}+1 \mathrm{e}^{-} \rightleftharpoons \operatorname{trans}-\left[\mathrm{Ru}(\mathrm{NO})\left(\mathrm{NH}_{3}\right)_{4} \mathrm{~L}\right)\right]^{2+} \\
& \text { trans } \left.\left.-\left[\mathrm{Ru}(\mathrm{NO})\left(\mathrm{NH}_{3}\right)_{4} \mathrm{~L}\right)\right]^{2+}+\mathrm{H}_{2} \mathrm{O} \stackrel{\mathrm{k}_{\mathrm{f}}}{\longrightarrow} \operatorname{trans}-\left[\mathrm{Ru}\left(\mathrm{H}_{2} \mathrm{O}\right)\left(\mathrm{NH}_{3}\right)_{4} \mathrm{~L}\right)\right]^{2+}+\mathrm{NO}
\end{aligned}
$$

Com base nestas observações, Nicholson e Shain [77] desenvolveram um método de medida cinética $\left(\mathrm{k}_{\mathrm{f}}\right)$ utilizando uma única varredura de onda cíclica, no qual a razão $\mathrm{i}_{\mathrm{pa}} / \mathrm{i}_{\mathrm{pc}}$ é dependente do potencial de inversão $\left(\mathrm{E}_{\lambda}\right)$ utilizado. Assim, foi construída uma curva de trabalho que correlaciona $i_{p a} / i_{p c}$ e $k_{f} \cdot \tau$, em que $\tau$ é o tempo em segundos entre $E_{1 / 2}$ e $E_{\lambda}$.

De acordo com a reação $5, \mathrm{k}_{\mathrm{f}}=\mathrm{k}_{-\mathrm{NO}}$, dessa forma a constante de liberação de NO foi calculada em $\mathrm{pH} 2,0, \mu=0,1 \mathrm{~mol} \mathrm{~L}^{-1}$ e $25 \pm 0,1^{\circ} \mathrm{C}$ por meio de uma única varredura de onda cíclica. Foram utilizadas velocidades de varreduras entre $20 \mathrm{mV} \mathrm{s}^{-1}$ a $2 \mathrm{~V} \mathrm{~s}^{-1} \mathrm{e}$, ao menos dois potenciais de inversão para cada complexo. Após obter o valor de $\mathrm{k}_{\mathrm{f}} \cdot \tau$, foi construído o gráfico de $\mathrm{k}_{\mathrm{f}}$. $\tau$ versus $\tau$ obtendo-se por meio do coeficiente angular o valor de $\mathrm{k}_{\text {-NO }}$.

Este método tem como vantagem o fato da razão entre as correntes de picos não depender de parâmetros experimentais como área do eletrodo e coeficientes de difusão das espécies eletroativas.

\subsubsection{Ressonância Magnética Nuclear}

Os espectros de Ressonância Magnética Nuclear (RMN) foram obtidos em espectrofotômetros BRUKER AC-200 (Central de Análises Químicas/IQSC) e Agilent 400/54 Premium Shielded. Foram utilizados óxido de deutério $\left(\mathrm{D}_{2} \mathrm{O}\right)$ como solvente, ácido 3(trimetilsilil)-2,2',3,3'-tetradeuteropropionico (TMSP-D4, $\left.\delta_{1 \mathrm{H}, 13 \mathrm{C}}=0 \mathrm{ppm}\right)$ e hexafluorfosfato 
de amônio $\left(\mathrm{NH}_{4} \mathrm{PF}_{6}, \delta_{31 \mathrm{P}}=-144 \mathrm{ppm}\right)$ como padrões internos para experimentos de ${ }^{1} \mathrm{H} /{ }^{13} \mathrm{C}$ e ${ }^{31} \mathrm{P}$, respectivamente.

\subsubsection{Difração de raios-X e determinação de estrutura}

As análises estruturais dos complexos trans-[Ru(NO) $\left.\left(\mathrm{NH}_{3}\right)_{4} \mathrm{P}(\mathrm{O})(\mathrm{OH})_{2}\right]\left(\mathrm{ZnCl}_{4}\right)$ e trans- $\left[\mathrm{Ru}(\mathrm{CO})\left(\mathrm{NH}_{3}\right)_{4}\left(\mathrm{P}(\mathrm{OH})_{3}\right)\right]\left(\mathrm{ZnCl}_{4}\right) \cdot \mathrm{H}_{2} \mathrm{O}$ foram realizadas no Instituto de Física de São Carlos pelo Prof. Dr. Eduardo Ernesto Castellano.

Os cristais foram selecionados e, então, montados em um difratômetro Enraf-Nonius Kappa-CCD com radiação MoKa monocromatizada por cristal de grafite. Os dados foram coletados a temperatura ambiente utilizando até $52^{\circ}$ em $2 \theta$ com redundância de aproximadamente 4 e parâmetros de cela unitária final baseados em todas as reflexões.

As coletas dos dados foram realizadas utilizando-se o programa COLLECT [78] a integração e o dimensionamento das reflexões foram efetuados com o sistema de programas HKL Denzo-Scalepack [79]. As correções de absorção foram feitas por meio do método de Gauss [80]. As estruturas foram resolvidas utilizando-se métodos diretos do programa SHELXS-97 [81]. Os modelos foram refinados por matriz de mínimos quadrados $\mathrm{F}^{2}$ usando SHELXL-97 [82]. Todos os átomos de hidrogênio foram posicionados estereoquimicamente e refinados com o modelo ridging. As Figuras 3 e 25 foram preparadas usando ORTEP-3 para Windows [83] com elipsoides de deslocamentos com probabilidades de 40-50\%. A listagem dos átomos coordenados e parâmetros de deslocamento isotópico equivalente, distâncias de ligação intramolecular e ângulos e parâmetros de anisotropia térmica foram disponibilizados por meio de depósito no Cambridge Crystallographic Data Centre (números de referência: CCDC 893741 e CCDC 841553). 


\subsubsection{Detalhes Computacionais}

Os cálculos foram realizados empregando-se o pacote do Gaussian03 [84]. As geometrias moleculares iniciais foram obtidas a partir de estruturas similares de trabalhos anteriores [51; 59] e otimizadas por meio da Teoria do Funcional da Densidade (DFT) de Kohn-Sham [85] com uso dos três parâmetros híbridos de troca-correlação funcional conhecidos como B3LYP (Becke, 3-parameter, Lee-Yang-Parr) [86; 87]. O conjunto de bases DGAUSS utilizado nos cálculos foi: DGDZVP (Degauss Duplo Zeta Valência Polarizada) para o rutênio, DGDZVP2 (Degauss Duplo Zeta Valência Polarizada 2) para o fósforo e DGTZVP (Degauss Triplo Zeta Valência Polarizada) para os demais átomos (C, H, N e O). Não foram impostas condições de simetria [88]. Todos os cálculos foram realizados utilizando o método de solvatação PCM (do inglês Polarizable Continuum Model) e água como solvente $[89 ; 90 ; 91]$.

Todos os mínimos de energia e os estados de transição (TS) foram caracterizados por cálculo de frequências, os quais apresentaram frequências reais para os mínimos de energia e uma frequência negativa para as estruturas do estado de transição. Para determinar o estado de transição para cada etapa da reação foi realizada uma varredura da superfície de energia potencial na coordenada de reação. A energia otimizada para os reagentes e produtos na superfície de energia potencial máxima foi tida como ponto de sela. Após obter as geometrias dos reagentes, produtos e o ponto de cela, foram realizados os cálculos de QST3 a fim de se ter a estrutura do estado de transição otimizada. A população dos orbitais foi examinada por meio da palavra chave pop $=$ full e os espectros eletrônicos foram obtidos utilizando-se cálculos de TD-DFT implementados no pacote Gaussian03. 
3.5 Hidrólise dos $\mathrm{P}(\mathrm{OR})_{3}=\mathbf{P}\left(\mathrm{OCH}_{3}\right)_{3}, \quad \mathbf{P}\left(\mathrm{OC}_{2} \mathrm{H}_{5}\right)_{3}, \quad \mathbf{P}\left(\mathrm{O}^{\mathrm{i}} \mathrm{C}_{3} \mathrm{H}_{7}\right)_{3}, \quad \mathbf{P}\left(\mathrm{OC}_{4} \mathrm{H}_{9}\right)_{3} \quad$ e $\mathrm{P}(\mathrm{OH})\left(\mathrm{OC}_{2} \mathrm{H}_{5}\right)_{2}$ livres e coordenados aos fragmentos trans- $\left[\mathrm{Ru}\left(\mathrm{H}_{2} \mathrm{O}\right)\left(\mathrm{NH}_{3}\right)_{4} \mathrm{~L}\right]^{2+}$ e trans $-\left[\mathrm{Ru}(\mathrm{NO})\left(\mathrm{NH}_{3}\right)_{4} \mathrm{~L}\right]^{3+}$

Os experimentos cinéticos envolvendo $\mathrm{RMN}\left({ }^{1} \mathrm{H}\right.$ ou $\left.{ }^{31} \mathrm{P}\right)$ foram realizados em sonda de $5 \mathrm{~mm}$ utilizando número de scans igual para todos os espectros e referência interna.

Os ésteres de fósforo livres e coordenados ao fragmento trans- $\left[\mathrm{Ru}\left(\mathrm{H}_{2} \mathrm{O}\right)\left(\mathrm{NH}_{3}\right)_{4} \mathrm{~L}\right]^{2+}$ foram analisados por meio de ${ }^{1} \mathrm{H}$ RMN devido à facilidade de aquisição e interpretação do espectro. Em adição, os sinais obtidos não apresentaram sobreposição, o que permitiu o cálculo da área em função do tempo.

Para os nitrosilos complexos foi utilizada a ${ }^{31} \mathrm{P}$ RMN em função do tempo a fim de evitar sobreposição de sinais, o que poderia prejudicar o cálculo de área dos mesmos.

O processamento de todos os espectros e os cálculos de área foram realizados com o programa KnowItAll(R) Informatic System 8.0.

As soluções $\mathrm{pH}$ 1,0, 3,0 e 7,5 foram preparadas em $\mathrm{D}_{2} \mathrm{O}$ utilizando ácido trifluoracético deuterado, ácido acético deuterado e tampão TRIS, respectivamente. A temperatura foi mantida a $25 \pm 0,5^{\circ} \mathrm{C}$.

3.6 Avaliação da estabilidade dos novos íons complexos trans- $\left[\mathrm{Ru}(\mathrm{NO})\left(\mathrm{NH}_{3}\right)_{4} \mathrm{P}(\mathrm{O})\left(\mathrm{OCH}_{2} \mathrm{CH}_{3}\right)_{2}\right]^{2+}$ e trans $-\left[\mathrm{Ru}(\mathrm{CO})\left(\mathrm{NH}_{3}\right)_{4} \mathrm{P}(\mathrm{OH})_{3}\right]^{2+}$

Os íns complexos trans- $\left[\mathrm{Ru}(\mathrm{NO})\left(\mathrm{NH}_{3}\right)_{4} \mathrm{P}(\mathrm{O})\left(\mathrm{OCH}_{2} \mathrm{CH}_{3}\right)_{2}\right]^{2+} \quad \mathrm{e}$ trans- $\left[\mathrm{Ru}(\mathrm{CO})\left(\mathrm{NH}_{3}\right)_{4} \mathrm{P}(\mathrm{OH})_{3}\right]^{2+}$ foram monitorados em função do tempo por meio das técnicas de ${ }^{31} \mathrm{P}$ RMN, voltamentria cíclica, espectroscopia vibracional e eletrônica em meio aquoso $\mathrm{pH} 3,0, \mathrm{pH} 7,5$ e $25^{\circ} \mathrm{C}$. 


\subsection{Determinação do $\mathrm{pK}_{\mathrm{a}}$ do éster de fosforo em trans- $\left[\mathrm{Ru}(\mathrm{CO})\left(\mathrm{NH}_{3}\right)_{4} \mathrm{P}(\mathrm{OH})_{3}\right]^{2+}$}

$\mathrm{O} \mathrm{pK}_{\mathrm{a}}$ do ácido fosforoso coordenado ao fragmento trans- $\left[\mathrm{Ru}(\mathrm{CO})\left(\mathrm{NH}_{3}\right)_{4}\right]^{2+}$ foi calculado por meio de espectroscopia vibracional em solução aquosa. Para tanto, foram efetuadas deconvoluções das $v_{\mathrm{CO}}$ considerando espectros obtidos na forma de absorbância. Para a aquisição dos espectros foi utilizado um espectrofotômetro BOMEM FTIR-102 e janelas de silício com espaçador de teflon de $0,10 \mathrm{~mm}$ e número de scans igual a 16 . Foram preparadas soluções tampão na faixa de $\mathrm{pH}$ de 1,60 a 4,50 com força tamponante de 200 mmol L $\mathrm{L}^{-1}$, uma vez que a concentração de complexo foi da ordem de $5 \times 10^{-2} \mathrm{~mol} \mathrm{~L}^{-1}$.

Para as deconvoluções espectrais foi utilizado como software o ORIGIN 7.5, de forma que os ajustes de curva não linear, utilizado para a deconvolução, apresentaram fator de correlação $\left(\mathrm{R}^{2}\right)$ de pelo menos 0,98 . A partir das deconvoluções foram obtidas as áreas sob a curva para cada uma das bandas nas diferentes concentrações hidrogeniônicas.

Dessa forma, foi calculada a porcentagem de cada uma das bandas, considerando a área da banda referente ao CO trans-posicionado ao ligante de ácido fosforoso protonado, $\mathrm{P}(\mathrm{OH})_{3}$, somada a área da banda referente ao $\mathrm{CO}$ trans-posicionado ao ácido fosforoso desprotonado, $\mathrm{P}(\mathrm{O})(\mathrm{OH})_{2}$, como área total. Em seguida, foi plotado o gráfico da porcentagem de área para cada banda pelo $\mathrm{pH}$ e o $\mathrm{pK}_{\mathrm{a}}$ obtido por meio da derivada primeira do mesmo. 


\section{RESULTADOS E DISCUSSÃO}

Esta seção foi dividida em quatro partes principais listadas a seguir:

- 4.1 Dissociação do ligante ácido fosforoso em trans- $\left[\mathrm{Ru}(\mathrm{NO})\left(\mathrm{NH}_{3}\right)_{4}\left(\mathrm{P}(\mathrm{OH})_{3}\right)\right]^{3+}$.

- 4.2 trans- $\left[\mathrm{Ru}(\mathrm{NO})\left(\mathrm{NH}_{3}\right)_{4}\left(\mathrm{P}(\mathrm{O})\left(\mathrm{OCH}_{2} \mathrm{CH}_{3}\right)_{2}\right)\right]\left(\mathrm{PF}_{6}\right)_{2}$ : síntese, caracterização e estabilidade em meio aquoso.

- 4.3 Estabilidade de ésteres de fósforo livres e coordenados a rutênio(II) em meio aquoso.

- 4.4 trans- $\left[\mathrm{Ru}(\mathrm{CO})\left(\mathrm{NH}_{3}\right)_{4}\left(\mathrm{P}(\mathrm{OH})_{3}\right)\right]^{2+}$ : síntese, caracterização e estabilidade em meio aquoso. 


\subsection{Dissociação do ligante de ácido fosforoso em trans- $\left[\mathrm{Ru}(\mathrm{NO})\left(\mathrm{NH}_{3}\right)_{4}\left(\mathrm{P}(\mathrm{OH})_{3}\right)\right]^{3+}$}

O ácido fosforoso é o modelo mais simples de éster de fósforo(III) e devido a isto foi utilizado coordenado ao fragmento trans- $\left[\mathrm{Ru}(\mathrm{NO})\left(\mathrm{NH}_{3}\right)_{4}\right]^{3+}$ a fim de se observar a reatividade deste sistema em meio aquoso. Em trabalho anterior [92], o complexo trans-[Ru(NO) $\left.\left(\mathrm{NH}_{3}\right)_{4}\left(\mathrm{P}(\mathrm{O})(\mathrm{OH})_{2}\right)\right] \mathrm{Cl}_{2}$ foi isolado e sua estabilidade em meio aquoso avaliada. No presente trabalho descreve-se uma nova rota de síntese que conduz ao sólido cristalino trans-[Ru(NO) $\left.\left(\mathrm{NH}_{3}\right)_{4}\left(\mathrm{P}(\mathrm{O})(\mathrm{OH})_{2}\right)\right]\left(\mathrm{ZnCl}_{4}\right)$ e o mecanismo para a dissociação do ácido fosforoso a partir do fragmento trans- $\left[\mathrm{Ru}(\mathrm{NO})\left(\mathrm{NH}_{3}\right)_{4}\right]^{3+}$.

\subsubsection{Difração de Raio-X: trans- $\left[\mathrm{Ru}(\mathrm{NO})\left(\mathrm{NH}_{3}\right)_{4}\left(\mathrm{P}(\mathrm{O})(\mathrm{OH})_{2}\right)\right]\left(\mathrm{ZnCl}_{4}\right) \cdot 3 \mathrm{H}_{2} \mathrm{O}$}

A estrutura cristalina do complexo é composta pelo cátion octaédrico trans- $\left[\mathrm{Ru}(\mathrm{NO})\left(\mathrm{NH}_{3}\right)_{4}\left(\mathrm{P}(\mathrm{O})(\mathrm{OH})_{2}\right)\right]^{2+}$ e pelo ânion $\left[\mathrm{ZnCl}_{4}\right]^{2^{-}}$(Figura 3). No cátion os quatro fragmentos $\mathrm{NH}_{3}$ ocupam posição equatorial e os grupos $\left[\mathrm{H}_{2} \mathrm{PO}_{3}\right]^{-}$e $\mathrm{NO}^{+}$estão trans-posicionados na direção axial. As principais distâncias e ângulos de ligação são apresentados na Tabela 2 .

Comparando-se os valores de distâncias e ângulos de ligação para o nitrosilo complexo de ácido fosforoso em questão e estes valores para o complexo trans- $\left[\mathrm{Ru}(\mathrm{NO})\left(\mathrm{NH}_{3}\right)_{4}\left(\mathrm{P}\left(\mathrm{OCH}_{2} \mathrm{CH}_{3}\right)_{3}\right)\right]^{3+}$ descritos na literatura [51], observa-se que, apenas são modificados o ângulo da ligação Ru-N-O de $175,1^{\circ}$ para $172.8^{\circ}$ e a distância de ligação Ru-NO de 1,774 para 1,793, do trans-[Ru(NO) $\left.\left.\left(\mathrm{NH}_{3}\right)_{4}\left(\mathrm{P}_{(\mathrm{OCH}} \mathrm{CH}_{3}\right)_{3}\right)\right]^{3+}$ para o trans-[Ru(NO) $\left.\left(\mathrm{NH}_{3}\right)_{4}\left(\mathrm{P}(\mathrm{O})(\mathrm{OH})_{2}\right)\right]^{2+}$, respectivamente. Esta alteração provavelmente deve-se ao fato do cristal do complexo de ácido fosforoso apresentar-se em sua forma desprotonada, o que favorece a retrodoação $\mathrm{Ru} \rightarrow \mathrm{NO}$, diminuindo a ordem de ligação $\mathrm{Ru}-\mathrm{NO}$ e deixando o $\mathrm{NO}^{+}$com mais características de $\mathrm{NO}^{0}$. 
Figura 3 - Representação ORTEP-3 da estrutura trans-[Ru(NO) $\left.\left(\mathrm{NH}_{3}\right)_{4}\left(\mathrm{P}(\mathrm{O})(\mathrm{OH})_{2}\right)\right] \mathrm{ZnCl}_{4}$ com elipsoides de deslocamentos com probabilidades de $50 \%$.
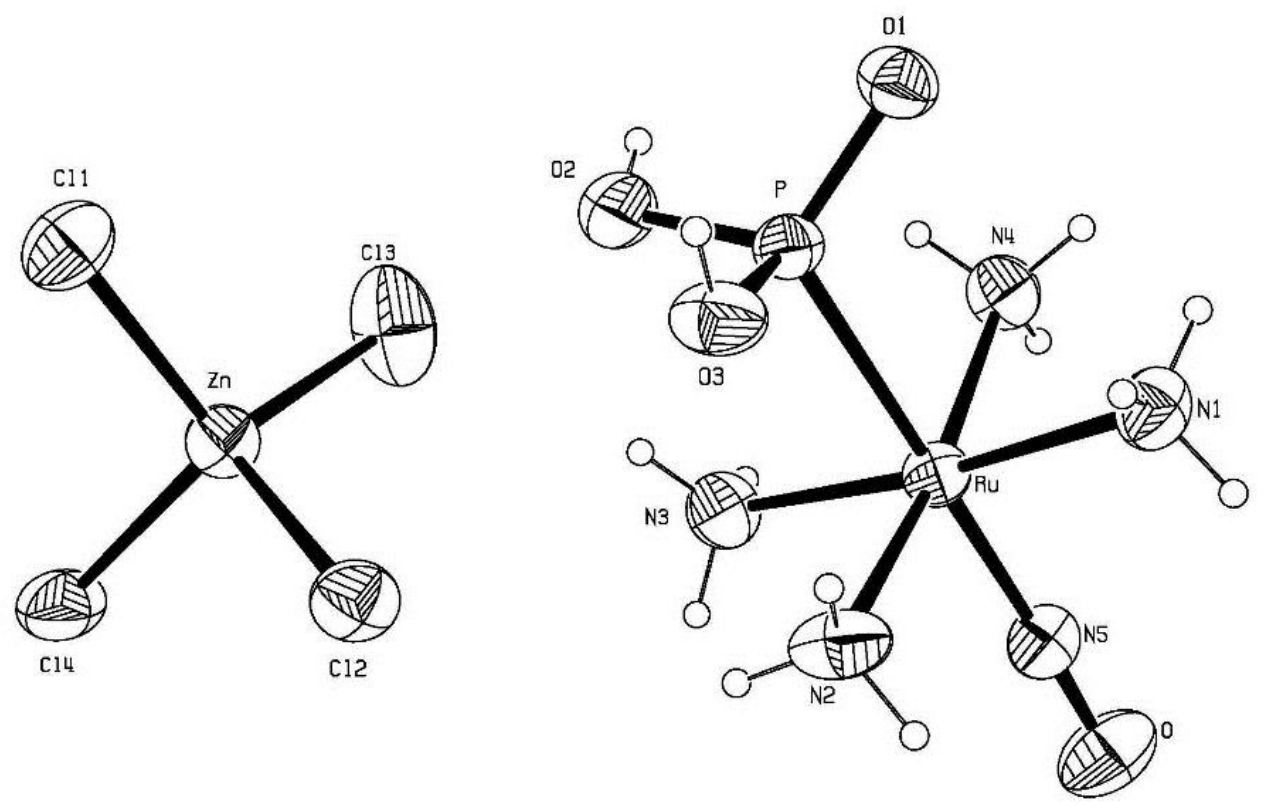

Fonte: Truzzi, D. R.; Ferreira, A. G.; Silva, S. C.; Castellano, E. E.; Lima , F. C. A.; Franco D. W. Nitrosyl induces phosphorous-acid dissociation in ruthenium(II). Dalton Transactions, v. 40, p. 12917-12925, 2011.

Tabela 1 - Dados cristalográficos para o trans-[Ru(NO)(NH$\left.)_{4}\left(\mathrm{P}(\mathrm{O})(\mathrm{OH})_{2}\right)\right] \mathrm{ZnCl}_{4}$

\begin{tabular}{ll}
\hline Fórmula & trans $-\left[\mathrm{Ru}(\mathrm{NO})\left(\mathrm{NH}_{3}\right)_{4}\left(\mathrm{P}(\mathrm{O})(\mathrm{OH})_{2}\right)\right] \mathrm{ZnCl}_{4}$ \\
\hline Grupo espacial & Monoclínico, $\mathrm{P}_{1} / \mathrm{n}$ \\
$a(\AA)$ & $7,0270(1)$ \\
$b(\AA)$ & $22,1460(8)$ \\
$c(\AA)$ & $9,2010(3)$ \\
$\beta($ deg $)$ & $91,499(2)$ \\
$Z$ & 4 \\
$V\left(\AA^{3}\right)$ & $1431,37(7)$ \\
$\lambda(\mathrm{Mo} \mathrm{K \alpha})(\AA)$ & 0,71073 \\
$\rho\left(\mathrm{mg} \mathrm{m}^{-3}\right)$ & 2,262 \\
$\mathrm{R} \mathrm{índices}$ & $\mathrm{R}_{1}=0,0493 ; \mathrm{R}_{2}=0,0992$ \\
\hline
\end{tabular}

Fonte: Truzzi, D. R.; Ferreira, A. G.; Silva, S. C.; Castellano, E. E.; Lima , F. C. A.; Franco D. W. Nitrosyl induces phosphorous-acid dissociation in ruthenium(II). Dalton Transactions, v. 40, p. 12917-12925, 2011. 
Tabela 2 - Distâncias e ângulos de ligação para $\mathrm{o}$ complexo trans- $\left[\mathrm{Ru}(\mathrm{NO})\left(\mathrm{NH}_{3}\right)_{4}\left(\mathrm{P}(\mathrm{O})(\mathrm{OH})_{2}\right)\right] \mathrm{ZnCl}_{4}$

\begin{tabular}{cccc}
\hline \multicolumn{4}{c}{ Distâncias de ligação $(\AA)$} \\
Ru-N(5) & $1,793(3)$ & $\mathrm{P}-\mathrm{O}(3)$ & $1,574(3)$ \\
$\mathrm{P}-\mathrm{O}(1)$ & $2,4136(10)$ & $\mathrm{P}-\mathrm{O}(2)$ & $1,579(3)$ \\
\multicolumn{4}{c}{ Ângulos de ligação $\left(^{\circ}\right)$} \\
\hline $\mathrm{N}(5)-\mathrm{Ru}-\mathrm{N}(3)$ & $93,78(14)$ & $\mathrm{N}(1)-\mathrm{Ru}-\mathrm{N}(4)$ & $90,25(14)$ \\
$\mathrm{N}(5)-\mathrm{Ru}-\mathrm{N}(2)$ & $90,55(14)$ & $\mathrm{N}(5)-\mathrm{Ru}-\mathrm{P}$ & $175,11(11)$ \\
$\mathrm{N}(3)-\mathrm{Ru}-\mathrm{N}(2)$ & $90,74(15)$ & $\mathrm{O}(1)-\mathrm{P}-\mathrm{O}(3)$ & $110,98(16)$ \\
$\mathrm{N}(5)-\mathrm{Ru}-\mathrm{N}(1)$ & $92,79(14)$ & $\mathrm{O}(1)-\mathrm{P}-\mathrm{O}(2)$ & $111,83(16)$ \\
$\mathrm{N}(3)-\mathrm{Ru}-\mathrm{N}(1)$ & $173,38(13)$ & $\mathrm{O}(3)-\mathrm{P}-\mathrm{O}(2)$ & $102,73(17)$ \\
$\mathrm{N}(2)-\mathrm{Ru}-\mathrm{N}(1)$ & $89,99(14)$ & $\mathrm{O}(1)-\mathrm{P}-\mathrm{Ru}$ & $113,71(11)$ \\
$\mathrm{N}(5)-\mathrm{Ru}-\mathrm{N}(4)$ & $97,64(14)$ & $\mathrm{O}(3)-\mathrm{P}-\mathrm{Ru}$ & $107,54(11)$ \\
$\mathrm{N}(3)-\mathrm{Ru}-\mathrm{N}(4)$ & $88,09(13)$ & $\mathrm{O}(2)-\mathrm{P}-\mathrm{Ru}$ & $109,41(13)$ \\
$\mathrm{N}(2)-\mathrm{Ru}-\mathrm{N}(4)$ & $171,79(13)$ & $\mathrm{O}-\mathrm{N}(5)-\mathrm{Ru}$ & $172,8(3)$ \\
\hline
\end{tabular}

Fonte: Truzzi, D. R.; Ferreira, A. G.; Silva, S. C.; Castellano, E. E.; Lima , F. C. A.; Franco D. W. Nitrosyl induces phosphorous-acid dissociation in ruthenium(II). Dalton Transactions, v. 40, p. 12917-12925, 2011.

\subsubsection{Mecanismo de dissociação do ácido fosforoso em} trans $-\left[\operatorname{Ru}(\mathrm{NO})\left(\mathrm{NH}_{3}\right)_{4}\left(\mathbf{P}(\mathrm{OH})_{3}\right)\right]^{3+}$

A estabilidade em meio aquoso do íon trans- $\left[\mathrm{Ru}(\mathrm{NO})\left(\mathrm{NH}_{3}\right)_{4}\left(\mathrm{P}(\mathrm{OH})_{3}\right)\right]^{3+} \quad\left(\delta_{31 \mathrm{P}}=68\right.$ ppm; $\left.v_{\mathrm{NO}+}=1892 \mathrm{~cm}^{-1}, \mathrm{pK}_{\mathrm{a}}=0,74\right)$ foi foco de estudo anterior [92] sendo observado experimentalmente que em $\mathrm{pH}=3,0$ sua forma desprotonada (trans $\left.-\left[\mathrm{Ru}(\mathrm{NO})\left(\mathrm{NH}_{3}\right)_{4}\left(\mathrm{P}(\mathrm{O})(\mathrm{OH})_{2}\right)\right]^{2+} ; \delta_{31 \mathrm{P}}=60 \mathrm{ppm} ; v_{\mathrm{NO}+}=1879 \mathrm{~cm}^{-1}\right)$ dá origem a duas novas espécies intermediárias $\left(\delta_{31 \mathrm{P}}=42\right.$ e $48 \mathrm{ppm}$ e $\left.v_{\mathrm{NO}+}=1874 \mathrm{~cm}^{-1}\right)$. Após a formação destas espécies foi observada a formação dos produtos trans- $\left[\mathrm{Ru}(\mathrm{NO})\left(\mathrm{NH}_{3}\right)_{4}\left(\mathrm{H}_{2} \mathrm{O}\right)\right]^{3+} \mathrm{e}$ $\mathrm{P}(\mathrm{OH})_{3}$ livre [92].

A dissociação do ácido fosforoso apresentou constantes de velocidade dependentes da concentração hidrogeniônica do meio $\left(\mathrm{k}=3,90 \times 10^{-7} \mathrm{em} \mathrm{pH} 0,6 ; \mathrm{k}=1,00 \times 10^{-6} \mathrm{em} \mathrm{pH} \mathrm{1,0;} \mathrm{k}\right.$ 
$=3,20 \times 10^{-5} \mathrm{em} \mathrm{pH} 2,0 ; \mathrm{k}=2,10 \times 10^{-4} \mathrm{em} \mathrm{pH} \mathrm{3,0)} \mathrm{[59;92].}$

Inicialmente as estruturas das duas espécies formadas após a desprotonação foram atribuídas com base no grau de blindagem do átomo de fósforo e na frequência de estiramento do $\mathrm{NO}^{+}$(Figura 4) [92]. Assim, foi proposto que a primeira espécie seria um complexo heptacoordenado (Figura 4 c), em que ambos os átomos, fósforo e oxigênio, estariam ligados ao centro metálico. Enquanto a segunda espécie seria um complexo hexa-coordenado no qual o éster de fósforo trivalente estaria coordenado apenas pelo átomo de oxigênio (Figura 4 d).

No entanto, os cálculos teóricos sugerem que a espécie hepta-coordenada trata-se de uma estrutura presente no estado de transição (Figura 5). Isto porque a varredura da superfície de energia potencial da reação de isomerização do ácido fosforoso coordenado pelo átomo de fósforo para o coordenado pelo átomo de oxigênio mostrou apenas um estado de transição. Este estado de transição apresenta justamente uma estrutura similar ao complexo hexacoordenado, para o qual se observa uma única frequência negativa referente à aproximação e ao afastamento dos átomos de fósforo e oxigênio da esfera de coordenação do $\mathrm{Ru}(\mathrm{II})$.

De acordo com os cálculos DFT, esta reação de isomerização se dá com uma energia de ativação $\left(\mathrm{E}_{\mathrm{a}}\right)$ de $31,1 \mathrm{kcal} \mathrm{mol}^{-1}$ (Figura 5), este valor está em concordância com uma reação que ocorre espontaneamente a temperatura ambiente. 
Figura 4 - Esquema proposto inicialmente para da dissociação do ligante de ácido fosforoso do fragmento trans-[Ru(NO) $\left.\left(\mathrm{NH}_{3}\right)_{4}\right]^{3+}[92]$

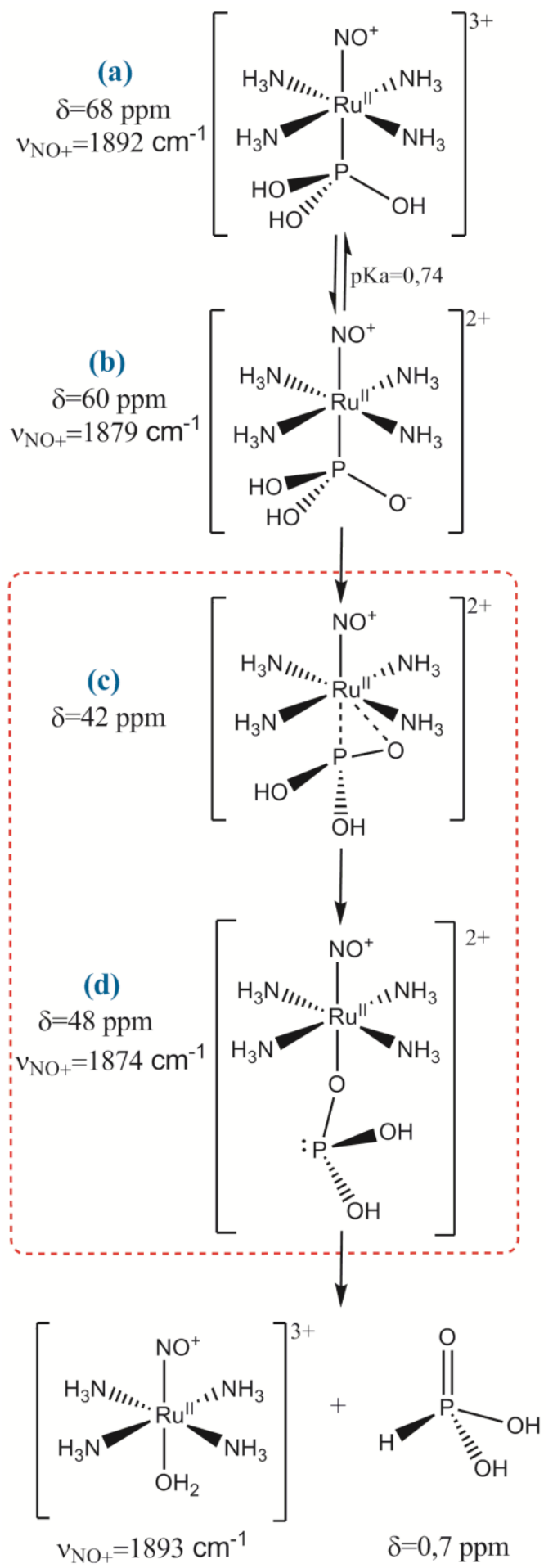


Figura 5 - Gráfico de energia $\left(\mathrm{kcal} \mathrm{mol}^{-1}\right)$ por coordenada intrínseca de reação (IRC) para a reação de isomerização do íon trans- $\left[\mathrm{Ru}(\mathrm{NO})\left(\mathrm{NH}_{3}\right)_{4}\left(\mathrm{P}(\mathrm{O})(\mathrm{OH})_{2}\right)\right]^{2+}$

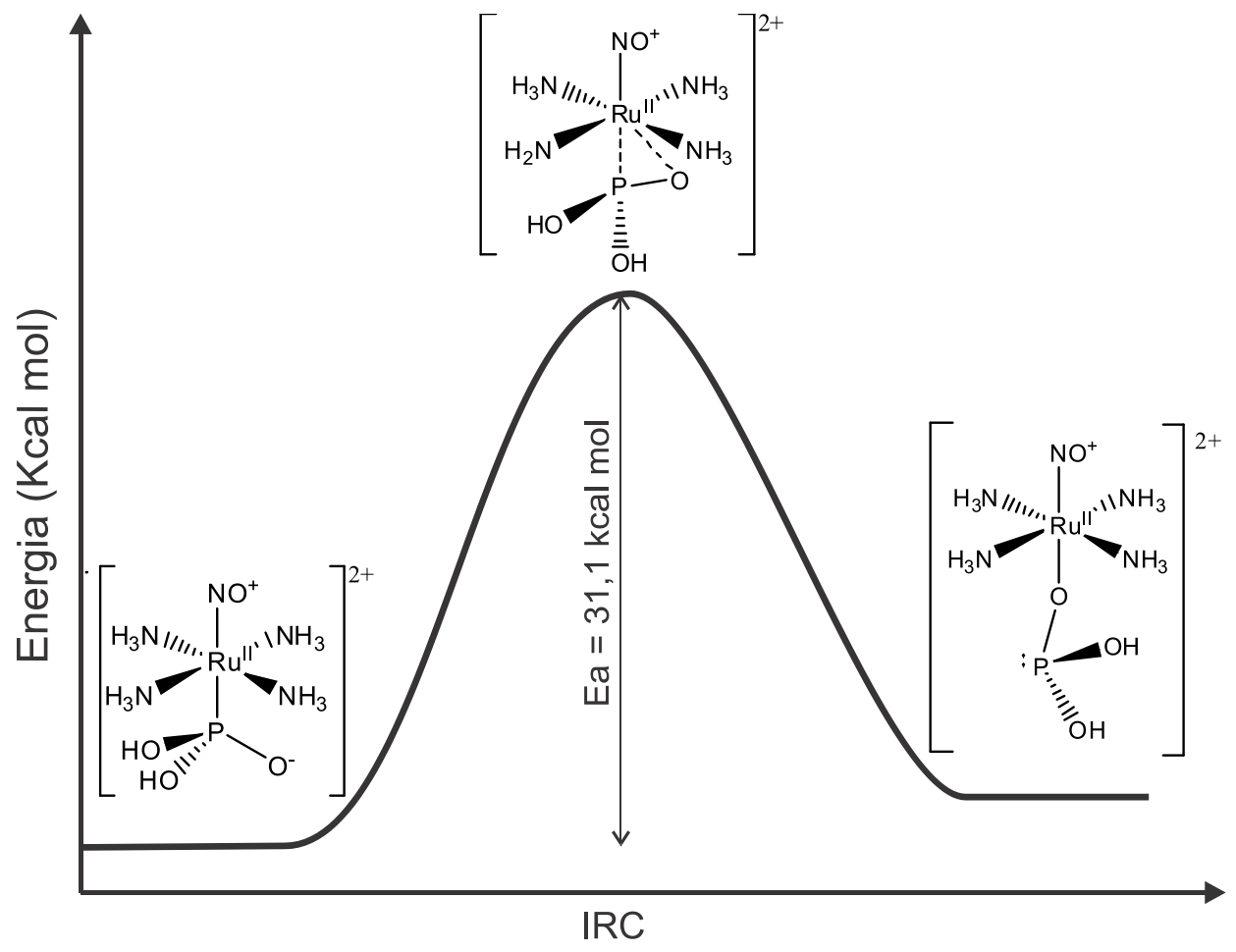

Dessa forma, sendo a espécie hepta-coordenada (Figura 4 c) uma estrutura do estado de transição e não um intermediário de reação, um novo mecanismo (Figura 6) foi proposto para a dissociação do ácido fosforoso com base nos dados experimentais e nos cálculos teóricos, o qual é descrito a seguir: Em solução o íon trans- $\left[\mathrm{Ru}(\mathrm{NO})\left(\mathrm{NH}_{3}\right)_{4}\left(\mathrm{P}(\mathrm{OH})_{3}\right)\right]^{3+}$ estabelece um rápido equilíbrio entre suas formas protonada e desprotonada (Figura 6 a e b). Estas formas são responsáveis pelos picos em 68 e 60 ppm, respectivamente. A partir do íon trans- $\left[\mathrm{Ru}(\mathrm{NO})\left(\mathrm{NH}_{3}\right)_{4}\left(\mathrm{P}(\mathrm{O})(\mathrm{OH})_{2}\right)\right]^{2+}$ (Figura 6 b) é formado um isômero de ligação, no qual o ácido fosforoso está ligado ao rutênio(II) pelo átomo de oxigênio desprotonado (trans- $\left[\mathrm{Ru}(\mathrm{NO})\left(\mathrm{NH}_{3}\right)_{4}\left((\mathrm{O}) \mathrm{P}(\mathrm{OH})_{2}\right)\right]^{2+}$, Figura $\left.6 \mathrm{c}\right)$. Nesta estrutura, o fósforo trivalente tem um par de elétrons livres que o blinda e faz com que o deslocamento químico do ${ }^{31} \mathrm{P}$ ocorra em 42 ppm. Neste isômero, o fósforo trivalente (Figura 6 c) não tem mais a estabilidade conferida pela retrodoação $\mathrm{Ru}(\mathrm{II}) \rightarrow \mathrm{P}(\mathrm{III})$, somando-se a isto o fato de que a forma mais estável do ligante de ácido fosforoso é a forma tetravalente (reação 3), ocorre a formação do 
íon trans- $\left[\mathrm{Ru}(\mathrm{NO})\left(\mathrm{NH}_{3}\right)_{4}\left((\mathrm{O}) \mathrm{P}(\mathrm{H})(\mathrm{OH})_{2}\right)\right]^{3+}$ (Figura 6 d), responsável pelo pico em 48 ppm. Por fim, o ácido fosforoso dissocia-se, observando-se a formação de ácido fosforoso livre $\left(\delta_{31 \mathrm{P}}=0,7 \mathrm{ppm}\right)$ e do íon trans $-\left[\mathrm{Ru}(\mathrm{NO})\left(\mathrm{NH}_{3}\right)_{4}\left(\mathrm{H}_{2} \mathrm{O}\right)\right]^{3+}\left(\mathrm{E}_{\mathrm{NO}}+/ \mathrm{NO} 0=-0,40 \mathrm{~V} ; v_{\mathrm{NO}}+=1893 \mathrm{~cm}^{-1}\right)$.

Figura 6 - Dissociação do ligante de ácido fosforoso a partir do complexo trans- $\left[\mathrm{Ru}(\mathrm{NO})\left(\mathrm{NH}_{3}\right)_{4}\left(\mathrm{P}(\mathrm{OH})_{3}\right)\right]^{3+}$
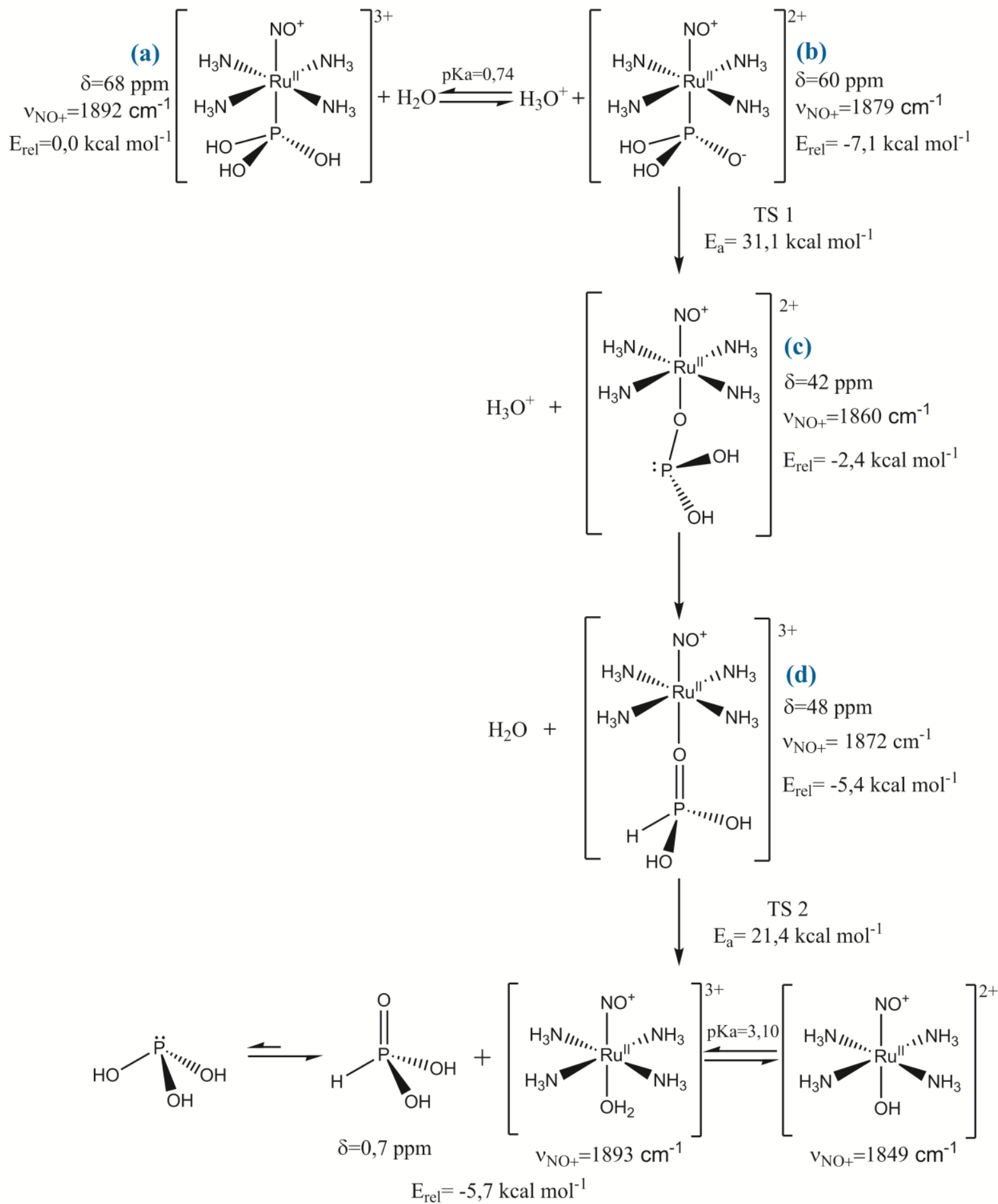
De acordo com os cálculos DFT, todas as espécies de nitrosilos complexo mostrados na Figura 6 exibem pequena variação em suas energias relativas $\left(\mathrm{E}_{\mathrm{rel}}\right)$. $\mathrm{O}$ estado de transição 1 (TS1) indicado na Figura 5, como descrito anteriormente, apresentou $\mathrm{E}_{\mathrm{a}}=31,1 \mathrm{kcal} \mathrm{mol}^{-1}$, enquanto o estado de transição 2 (TS2) que ocorre durante a dissociação do ácido fosforoso e a consequente coordenação da água apresentou $\mathrm{E}_{\mathrm{a}}=21,4 \mathrm{kcal} \mathrm{mol}^{-1}$. A partir da sequência de reações consecutivas proposta na Figura 6, é difícil estabelecer a etapa lenta da reação tendo como base apenas os dados de constante de velocidade [92]. Entretanto, as energias de ativação obtidas nos cálculos DFT sugerem que a etapa lenta da reação seja a isomerização do $\mathrm{Ru}(\mathrm{II})-\mathrm{P}(\mathrm{III})$ para o Ru(II)-O (Figura 6 b e c).

O cálculo das cargas de Mulliken (Tabela 3) mostra que a desprotonação do ácido fosforoso causa um decréscimo da carga positiva sobre o átomo de fósforo (de 1,296 para 1,282), a qual é consistente com os picos em 68 e 60 ppm no espectro de ${ }^{31} \mathrm{P}$ RMN. A estrutura (c) apresenta a carga do fósforo menos positiva do que a da estrutura (d), o que também está de acordo com as atribuições de ${ }^{31} \mathrm{P}$ RMN.

Tabela 3 - Cargas atômicas de Mulliken calculadas para os complexos de ácido fosforoso

\begin{tabular}{ccccc}
\hline \multirow{2}{*}{ Átomos } & \multicolumn{4}{c}{ Estruturas } \\
& (a) & (b) & (c) & (d) \\
\hline Ru & 0,185 & 0,205 & 0,741 & 0,699 \\
O (nitrosônio) & 0,122 & 0,118 & 0,082 & 0,103 \\
N (nitrosônio) & 0,153 & 0,141 & 0,123 & 0,143 \\
P & 1,296 & 1,282 & 0,858 & 1,218 \\
O (protonado) & $-0,590$ & $-0,625$ & $-0,655$ & $-0,674$ \\
O (desprotonado) & & $-0,736$ & $-0,692$ & $-0,615$ \\
\hline
\end{tabular}

Fonte: Truzzi, D. R.; Ferreira, A. G.; Silva, S. C.; Castellano, E. E.; Lima , F. C. A.; Franco D. W. Nitrosyl induces phosphorous-acid dissociation in ruthenium(II). Dalton Transactions, v. 40, p. 12917-12925, 2011.

Os valores de $v_{\mathrm{NO}^{+}}$de 1969,1961 e $1963 \mathrm{~cm}^{-1}$ foram calculados teoricamente para as estruturas (a), (b) e (d) da Figura 6, respectivamente, e estão de acordo com a sequência 
obtida experimentalmente $\left(1892,1879\right.$ e $\left.1872 \mathrm{~cm}^{-1}\right)$. O valor de $v_{\mathrm{NO}^{+}}$para a estrutura (c) da Figura 6 foi calculada como igual a $1947 \mathrm{~cm}^{-1}$. O $v_{\mathrm{NO}}+$ para esta última estrutura não foi nitidamente observado experimentalmente devido à sobreposição de bandas. Entretanto, uma análise mais cuidadosa do espectro da Figura 7 (linha cheia) permite observar a presença de um ombro em $1860 \mathrm{~cm}^{-1}$.

Figura 7 - Espectro de infravermelho do íon trans- $\left[\mathrm{Ru}(\mathrm{NO})\left(\mathrm{NH}_{3}\right)_{4}\left(\mathrm{P}(\mathrm{OH})_{3}\right)\right]^{3+}$ em solução $\mathrm{pH}=3,0, \mu=0,10 \mathrm{~mol} \mathrm{~L}{ }^{-1}, \mathrm{C}_{\mathrm{Ru}}=5,0 \times 10^{-2} \mathrm{~mol} \mathrm{~L}^{-1}$ e espaçador de $0,10 \mathrm{~mm}$. Linha pontilhada: $\mathrm{t}=5 \mathrm{~min}$. Linha cheia: $\mathrm{t}=1 \mathrm{~h}$

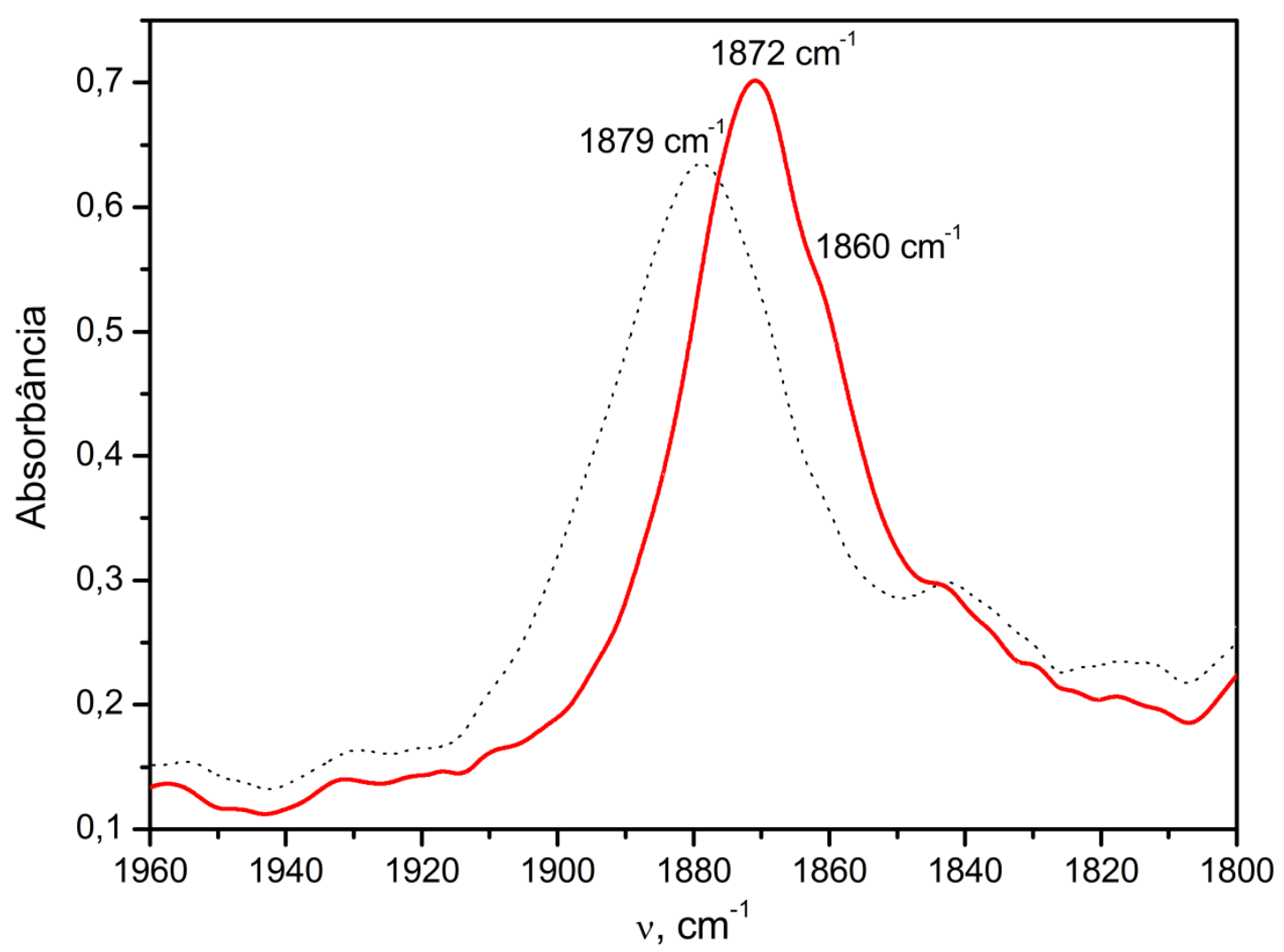

O cálculo de TD-DFT (Teoria do Funcional de Densidade Dependente do Tempo) para a sequência de reações da Figura 6 ajuda a explicar as mudanças observadas no espectro eletrônico do íon trans-[Ru(NO)( $\left.\left(\mathrm{NH}_{3}\right)_{4}\left(\mathrm{P}(\mathrm{OH})_{3}\right)\right]^{3+}$ em função do tempo em meio aquoso (Figura 8 a). 
Figura 8 - (a) Espectro eletrônico experimental do íon trans- $\left[\mathrm{Ru}(\mathrm{NO})\left(\mathrm{NH}_{3}\right)_{4}\left(\mathrm{P}(\mathrm{OH})_{3}\right)\right]^{3+}$ em solução $\mathrm{pH}=3,0, \mu=0,10 \mathrm{~mol} \mathrm{~L}{ }^{-1}, 25 \pm 0,1^{\circ} \mathrm{C}$ e $\mathrm{C}_{\mathrm{Ru}}=3,70 \times 10^{-4} \mathrm{~mol} \mathrm{~L}^{-1}$ com intervalo de tempo de 5 a 20 minutos (Espectro inserido: 20 a 30 minutos); (b) Espectro eletrônico teórico para cada um dos nitrosilos complexos de ácido fosforoso.
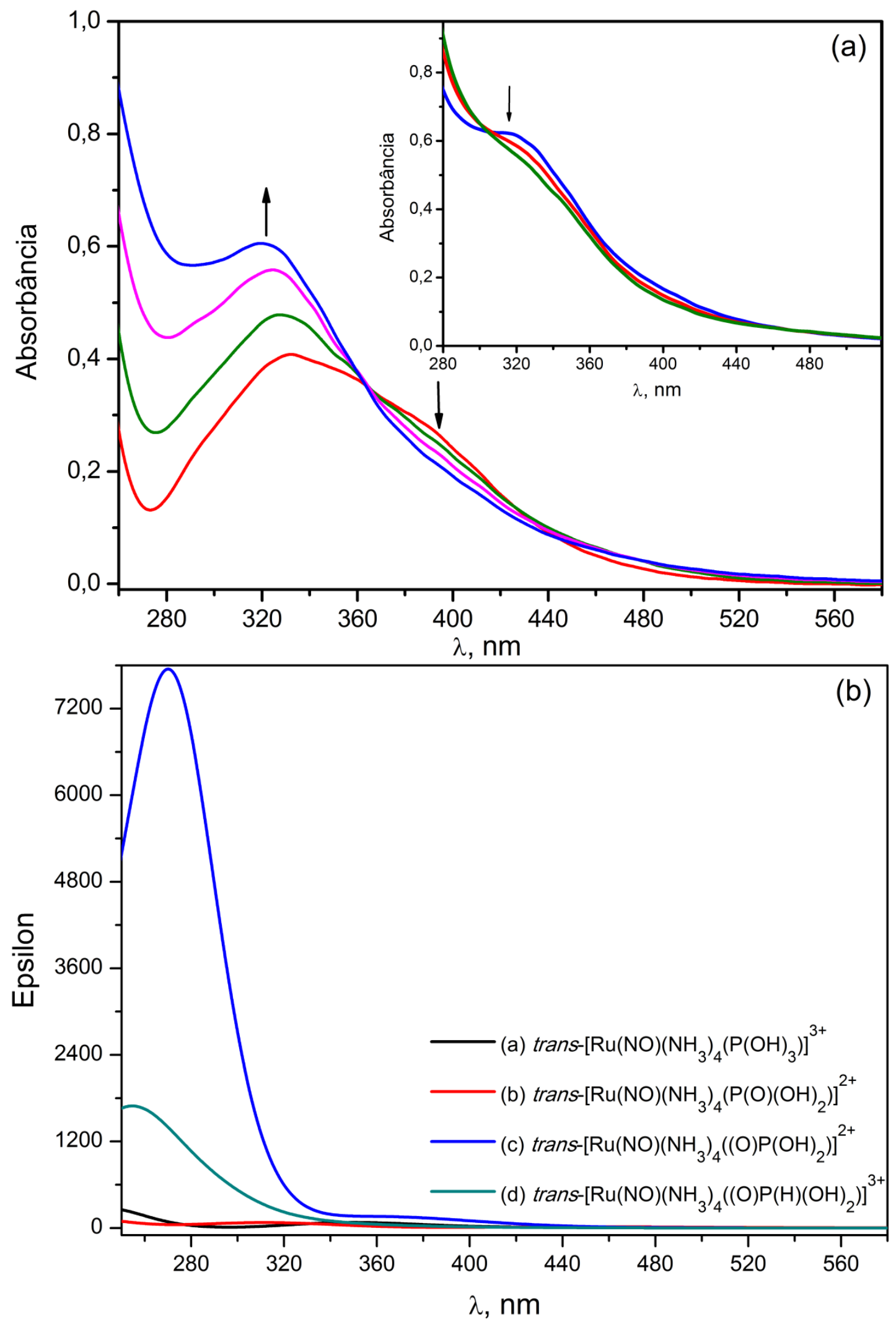
As espécies trans- $\left[\mathrm{Ru}(\mathrm{NO})\left(\mathrm{NH}_{3}\right)_{4}\left(\mathrm{P}(\mathrm{OH})_{3}\right)\right]^{3+}$ e trans- $\left[\mathrm{Ru}(\mathrm{NO})\left(\mathrm{NH}_{3}\right)_{4}\left(\mathrm{P}(\mathrm{O})(\mathrm{OH})_{2}\right)\right]^{2+}$ apresentam pequenas diferenças em seus espectros eletrônicos simulados (Figura 8 b). No entanto, o espectro do trans-[Ru(NO) $\left.\left(\mathrm{NH}_{3}\right)_{4}\left((\mathrm{O}) \mathrm{P}(\mathrm{OH})_{2}\right)\right]^{2+}$, no qual o oxigênio com carga negativa está diretamente ligado ao metal, apresenta um aumento considerável da intensidade na região de $300 \mathrm{~nm}$, o qual é principalmente atribuído às transições do tipo TCLL (transferência de carga ligante-ligante) e TCLM (transferência de carga ligante-metal), as quais são esperadas quando o átomo de oxigênio está ligado diretamente ao metal. A intensidade desta banda diminui quando o íon trans- $\left[\mathrm{Ru}(\mathrm{NO})\left(\mathrm{NH}_{3}\right)_{4}\left((\mathrm{O}) \mathrm{P}(\mathrm{H})(\mathrm{OH})_{2}\right)\right]^{3+}$ é formado. Essas modificações nos valores de absorbância calculadas estão de acordo com o observado experimentalmente em função do tempo.

Basallote et al [93] sugerem com base em cálculos teóricos a formação dos isômeros de ácido fosforoso coordenado pelo átomo de oxigênio desprotonado tanto na forma trivalente quanto na forma tetravalente em clusters de molibdênio/paládio e molibdênio/níquel. No entanto, nenhuma das espécies foi observada experimentalmente.

Em tetraaminas de rutênio, as propriedades cinéticas e termodinâmicas do fragmento $\left[\mathrm{RuNO}^{3+}\right.$ assemelham-se às do centro de Ru(III) [94; 95]. Taube et al [96] demonstraram que para o íon $\left[\mathrm{Ru}\left(\mathrm{NH}_{3}\right)_{5}\left(\mathrm{Me}_{2} \mathrm{SO}\right)\right]^{\mathrm{n}+}$ o complexo ligado pelo oxigênio é mais estável em $\mathrm{Ru}(\mathrm{III})$, enquanto que o ligado pelo enxofre é mais estáveis em $\mathrm{Ru}(\mathrm{II})$. Dessa forma, a isomerização de ligação do ácido fosforoso desprotonado do P(III) para o O é concebível. De forma similar ao observado para o trans-[Ru(NO) $\left.\left(\mathrm{NH}_{3}\right)_{4}\left(\mathrm{H}_{2} \mathrm{O}\right)\right]^{3+}$ [95], o ligante nitrosônio pode induzir a doação de elétrons $\mathrm{p}$ do oxigênio para $\mathrm{o} \quad \mathrm{Ru}(\mathrm{II}) \mathrm{d} \pi \quad \operatorname{nos}$ íons trans- $\left[\mathrm{Ru}(\mathrm{NO})\left(\mathrm{NH}_{3}\right)_{4}\left((\mathrm{O}) \mathrm{P}(\mathrm{OH})_{2}\right)\right]^{2+}$ e trans- $\left[\mathrm{Ru}(\mathrm{NO})\left(\mathrm{NH}_{3}\right)_{4}\left((\mathrm{O}) \mathrm{P}(\mathrm{H})(\mathrm{OH})_{2}\right)\right]^{2+}$.

Esta proposta de isomerização explica a dependência entre a constante de dissociação do ácido fosforoso e o $\mathrm{pH}$, pois a formação de $\operatorname{trans}-\left[\mathrm{Ru}(\mathrm{NO})\left(\mathrm{NH}_{3}\right)_{4}\left((\mathrm{O}) \mathrm{P}(\mathrm{OH})_{2}\right)\right]^{2+}$ é dependente da concentração da espécie trans- $\left[\mathrm{Ru}(\mathrm{NO})\left(\mathrm{NH}_{3}\right)_{4}\left(\mathrm{P}(\mathrm{O})(\mathrm{OH})_{2}\right)\right]^{2+}\left(\mathrm{pK}_{\mathrm{a}}=0,74\right)$. 
O íon trans- $\left[\mathrm{Ru}(\mathrm{NO})\left(\mathrm{NH}_{3}\right)_{4}\left((\mathrm{O}) \mathrm{P}(\mathrm{OH})_{2}\right)\right]^{2+}$ (Figura 6 c) poderia ser útil como modelo para estudos de transferência de fósforo, pois nesta estrutura o átomo de fósforo disponibiliza um par de elétrons que poderia promover modificações químicas.[97; 98]. Esse tipo de reação é relevante na química medicinal para obter derivados fosforados com propriedades fisiológicas $[97 ; 98]$ 


\section{2 trans $-\left[\mathrm{Ru}(\mathrm{NO})\left(\mathrm{NH}_{3}\right)_{4}\left(\mathrm{P}(\mathrm{O})\left(\mathrm{OCH}_{2} \mathrm{CH}_{3}\right)_{2}\right)\right]\left(\mathrm{PF}_{6}\right)_{2}: \quad$ síntese, caracterização} estabilidade em meio aquoso.

\subsubsection{Caracterização do sólido trans- $\left[\mathrm{Ru}(\mathrm{NO})\left(\mathrm{NH}_{3}\right)_{4}\left(\mathrm{P}(\mathrm{O})\left(\mathrm{OCH}_{2} \mathrm{CH}_{3}\right)_{2}\right)\right]\left(\mathrm{PF}_{6}\right)_{2}$}

A síntese do nitrosilo complexo de dietil fosfito deu origem a um sólido de cor rosa. Os resultados de Análise Elementar de carbono, nitrogênio e hidrogênio encontram-se na Tabela 4 e concordam com as porcentagens teóricas calculadas para este complexo.

Tabela 4 - Dados de Análise Elementar

\begin{tabular}{cccc}
\hline & Teórico (\%) & Experimental (\%) & Erro (\%) \\
\hline trans- $\left[\mathrm{Ru}(\mathrm{NO})\left(\mathrm{NH}_{3}\right)_{4}\left(\mathrm{P}(\mathrm{O})\left(\mathrm{OCH}_{2} \mathrm{CH}_{3}\right)_{2}\right)\right]\left(\mathrm{PF}_{6}\right)_{2} \cdot$ & $1 / 2 \mathrm{CH}_{3} \mathrm{CH}_{2} \mathrm{OH}$ \\
$\mathbf{C}$ & 9,25 & 9,23 & 0,2 \\
$\mathbf{H}$ & 3,88 & 3,71 & 4,3 \\
$\mathbf{N}$ & 11,09 & 11,05 & 0,3 \\
\hline
\end{tabular}

Considerando que no fragmento $[\mathrm{Ru}(\mathrm{NO})]^{3+}$, o centro metálico exibe caráter de $\mathrm{Ru}(\mathrm{III}), \mathrm{o} \mathrm{pK}_{\mathrm{a}}$ do dietil fosfito coordenado a este fragmento pode ser estimado com valor de ordem de grandeza similar ao apresentado pelo dietil fosfito coordenado ao fragmento $\left[\mathrm{Ru}\left(\mathrm{H}_{2} \mathrm{O}\right)\left(\mathrm{NH}_{3}\right)_{4}\right]^{3+}\left(\mathrm{pK}_{\mathrm{a}}=1,5\right)$ [56]. Este comportamento foi previamente observado para os nitrosilo complexos trans-[Ru(NO) $\left.\left(\mathrm{NH}_{3}\right)_{4}\left(\mathrm{H}_{2} \mathrm{O}\right)\right]^{3+}[95]$ e trans- $\left[\mathrm{Ru}(\mathrm{NO})\left(\mathrm{NH}_{3}\right)_{4}\left(\mathrm{P}(\mathrm{OH})_{3}\right)\right]^{3+}$ [59] e seus respectivos aquo-complexos de rutênio(III). Dessa forma, como padronização, sempre que o nitrosilo complexo de dietil fosfito for mencionado em meio aquoso em $\mathrm{pH}>$ 1,5 este ligante será representado em sua forma desprotonada. 


\subsubsection{Espectroscopia Eletrônica.}

$\mathrm{O}$ espectro eletrônico do íon complexo trans- $\left[\mathrm{Ru}(\mathrm{NO})\left(\mathrm{NH}_{3}\right)_{4}\left(\mathrm{P}(\mathrm{OH})\left(\mathrm{OCH}_{2} \mathrm{CH}_{3}\right)_{2}\right)\right]^{3+}$ em solução $\mathrm{pH} 1,0, \mu=0,1 \mathrm{~mol} \mathrm{~L}^{-1}$ (Figura 9) apresenta bandas em $\lambda \sim 530 \mathrm{~nm}$ $\left(\varepsilon \sim 20 \mathrm{~L} \mathrm{~mol}^{-1} \mathrm{~cm}^{-1}\right), 310 \mathrm{~nm}\left(\varepsilon=1003 \mathrm{~L} \mathrm{~mol}^{-1} \mathrm{~cm}^{-1}\right)$ e $242 \mathrm{~nm}\left(\varepsilon=6250 \mathrm{~L} \mathrm{~mol}^{-1} \mathrm{~cm}^{-1}\right) . \mathrm{O}$ espectro em linhas gerais assemelha-se ao observado para os outros compostos da série trans-[Ru(NO) $\left.\left(\mathrm{NH}_{3}\right)_{4} \mathrm{~L}\right] \mathrm{X}_{\mathrm{n}}[99 ; 100]$.

Figura 9 - Espectro eletrônico do íon trans- $\left[\mathrm{Ru}(\mathrm{NO})\left(\mathrm{NH}_{3}\right)_{4}\left(\mathrm{P}(\mathrm{OH})\left(\mathrm{OCH}_{2} \mathrm{CH}_{3}\right)_{2}\right)\right]^{3+}$ em solução pH 1,0, $\mu=0,10 \mathrm{~mol} \mathrm{~L}{ }^{-1} \mathrm{CF}_{3} \mathrm{COONa}, 25 \pm 0,1^{\circ} \mathrm{C}$ (a) $\mathrm{C}_{\mathrm{Ru}}=2,08 \times 10^{-4} \mathrm{~mol} \mathrm{~L}^{-1} \mathrm{e}$ cubeta de $1,0 \mathrm{~cm}$ de caminho ótico, (b) $\mathrm{C}_{\mathrm{Ru}}=1,28 \times 10^{-3} \mathrm{~mol} \mathrm{~L}^{-1}$ e cubeta de $5,0 \mathrm{~cm}$ de caminho ótico.

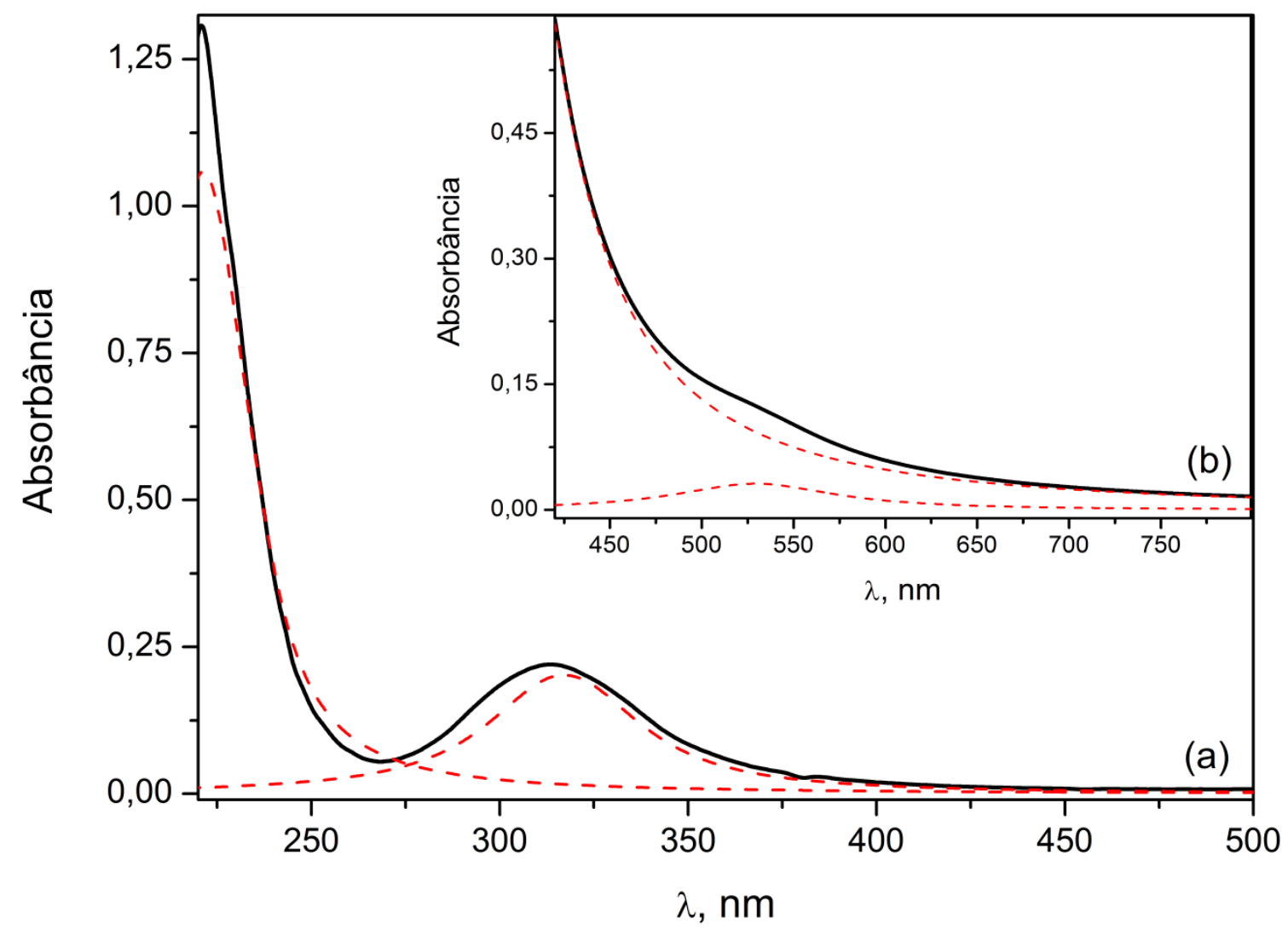

O cálculo teórico da população dos orbitais moleculares deste complexo mostram que o HOMO é composto de $100 \% \mathrm{Ru}\left(\mathrm{d}_{\mathrm{xy}}\right)$, enquanto que o LUMO e LUMO+1 são compostos 
por $71 \%$ NO $\left(p_{x}\right.$ e $\left.p_{y}, \pi^{*}\right)$ e $26 \% R u\left(d_{x z}\right.$ e $\left.d_{y z}\right)$. De acordo com os cálculos (TD-DFT), a banda em $530 \mathrm{~nm}$ resulta da transição $\operatorname{HOMO}\left(\mathrm{d}_{\mathrm{xy}} \mathrm{Ru}\right) \rightarrow \mathrm{LUMO}+0,1\left(\pi^{*} \mathrm{NO}\right)$, ou seja, de uma TCML (transferência de carga metal-ligante). A absorção em $310 \mathrm{~nm}$ é atribuída a transição d-d do tipo $\operatorname{HOMO}\left(\mathrm{d}_{\mathrm{xy}} \mathrm{Ru}\right) \rightarrow \mathrm{LUMO}+2\left(\mathrm{~d}_{\mathrm{z}} 2 \mathrm{Ru}\right)$ e a transferência de carga HOMO-3,4 $\left(\mathrm{d}_{\mathrm{xz}}, \mathrm{d}_{\mathrm{yz}}\right.$ $\mathrm{Ru} \rightarrow \mathrm{LUMO}+0,1\left(\pi^{*} \mathrm{NO}\right)$. Ao passo que a absorção em $242 \mathrm{~nm}$ apresenta uma maior composição de TCML (HOMO-1,4( $\left.\left.\mathrm{d}_{\mathrm{z}^{2}}, \mathrm{~d}_{\mathrm{xy}} \mathrm{Ru}\right) \rightarrow \mathrm{LUMO}+0,1(\pi * \mathrm{NO})\right)$ e uma contribuição menor do tipo TCLL (HOMO-2(pP(OH) $\left.\left.\left(\mathrm{OCH}_{2} \mathrm{CH}_{3}\right)_{2}\right) \rightarrow \mathrm{LUMO}+1(\pi * \mathrm{NO})\right)$.

\subsubsection{Voltametria Cíclica}

O voltamograma para a solução contendo o íon trans- $\left[\mathrm{Ru}(\mathrm{NO})\left(\mathrm{NH}_{3}\right)_{4}\left(\mathrm{P}(\mathrm{O})\left(\mathrm{OCH}_{2} \mathrm{CH}_{3}\right)_{2}\right)\right]^{2+}$ é mostrado na Figura 10 a.

$\mathrm{O}$ ligante nitrosônio $\left(\mathrm{NO}^{+}\right)$é reduzido em $\mathrm{E}_{\mathrm{pc}}=-0,50 \mathrm{~V}$ vs $\mathrm{ECS}$ (reação 6) e o ligante óxido nítrico $(\mathrm{NO})$ é reduzido em $\mathrm{E}_{\mathrm{pc}}=-0,80 \mathrm{~V}$ vs ECS (reação 7). Os correspondentes picos de oxidação são apenas parcialmente observados em $\mathrm{E}_{\mathrm{pa}}=-0,42 \mathrm{~V}$ e $\mathrm{E}_{\mathrm{pa}}=-0,63 \mathrm{~V}$ vs $\mathrm{ECS}$, respectivamente. Isto devido ao elevado efeito trans que o ligante de fósforo apresenta, o que conduz a uma rápida liberação dos grupos $\mathrm{NO}$ e $\mathrm{NO}^{-}$gerados por redução. O par reversível observado em 0,29 V vs ECS é atribuído ao processo de óxido-redução dos centros de $\mathrm{Ru}^{\mathrm{III}} / \mathrm{Ru}^{\mathrm{II}}$ do aquo-complexo (reação 8).

trans- $\left[\mathrm{Ru}\left(\mathrm{NO}^{+}\right)\left(\mathrm{NH}_{3}\right)_{4} \mathrm{P}(\mathrm{OH})\left(\mathrm{OCH}_{2} \mathrm{CH}_{3}\right)_{2}\right]^{3+} \underset{-\mathrm{e}^{-}}{\stackrel{+\mathrm{e}^{-}}{-}}$trans- $\left[\mathrm{Ru}(\mathrm{NO})\left(\mathrm{NH}_{3}\right)_{4} \mathrm{P}(\mathrm{OH})\left(\mathrm{OCH}_{2} \mathrm{CH}_{3}\right)_{2}\right]^{2+}$

trans- $\left[\mathrm{Ru}(\mathrm{NO})\left(\mathrm{NH}_{3}\right)_{4} \mathrm{P}(\mathrm{OH})\left(\mathrm{OCH}_{2} \mathrm{CH}_{3}\right)_{2}\right]^{2+} \stackrel{+\mathrm{e}^{-}}{\underset{-\mathrm{e}^{-}}{-}}$trans- $\left[\mathrm{Ru}\left(\mathrm{NO}^{-}\right)\left(\mathrm{NH}_{3}\right)_{4} \mathrm{P}(\mathrm{OH})\left(\mathrm{OCH}_{2} \mathrm{CH}_{3}\right)_{2}\right]^{+}$

trans- $\left[\mathrm{Ru}\left(\mathrm{H}_{2} \mathrm{O}\right)\left(\mathrm{NH}_{3}\right)_{4} \mathrm{P}(\mathrm{OH})\left(\mathrm{OCH}_{2} \mathrm{CH}_{3}\right)_{2}\right]^{3+} \stackrel{+\mathrm{e}^{-}}{\underset{-\mathrm{e}^{-}}{\rightleftharpoons}}$ trans- $\left[\mathrm{Ru}\left(\mathrm{H}_{2} \mathrm{O}\right)\left(\mathrm{NH}_{3}\right)_{4} \mathrm{P}(\mathrm{OH})\left(\mathrm{OCH}_{2} \mathrm{CH}_{3}\right)_{2}\right]^{2+}$ 
A Figura 10 b mostra um voltamograma de pulso diferencial do trans- $\left[\mathrm{Ru}(\mathrm{NO})\left(\mathrm{NH}_{3}\right)_{4}\left(\mathrm{P}(\mathrm{O})\left(\mathrm{OCH}_{2} \mathrm{CH}_{3}\right)_{2}\right)\right]^{2+}$, no qual os processos de redução aparecem de forma mais definida devido a maior sensibilidade e resolução conferida por esta técnica.

Figura 10 - (a) Voltamograma cíclico para o íon trans- $\left[\mathrm{Ru}(\mathrm{NO})\left(\mathrm{NH}_{3}\right)_{4}\left(\mathrm{P}(\mathrm{OH})\left(\mathrm{OCH}_{2} \mathrm{CH}_{3}\right)_{2}\right)\right]^{3+}$ em meio aquoso $\mathrm{pH} 1,0 ; \mu=0,1 \mathrm{~mol} \mathrm{~L}^{-1} ; \mathrm{C}_{\mathrm{Ru}}=1,5 \times 10^{-3} \mathrm{~mol} \mathrm{~L}^{-1} ; \mathrm{T}=5 \pm 0,1^{\circ} \mathrm{C}$; velocidade $=$ $100 \mathrm{mV} \mathrm{s}^{-1}$. (b) Voltamograma de pulso diferencial realizado a velocidade de $20 \mathrm{mV} \mathrm{s}^{-1}$.

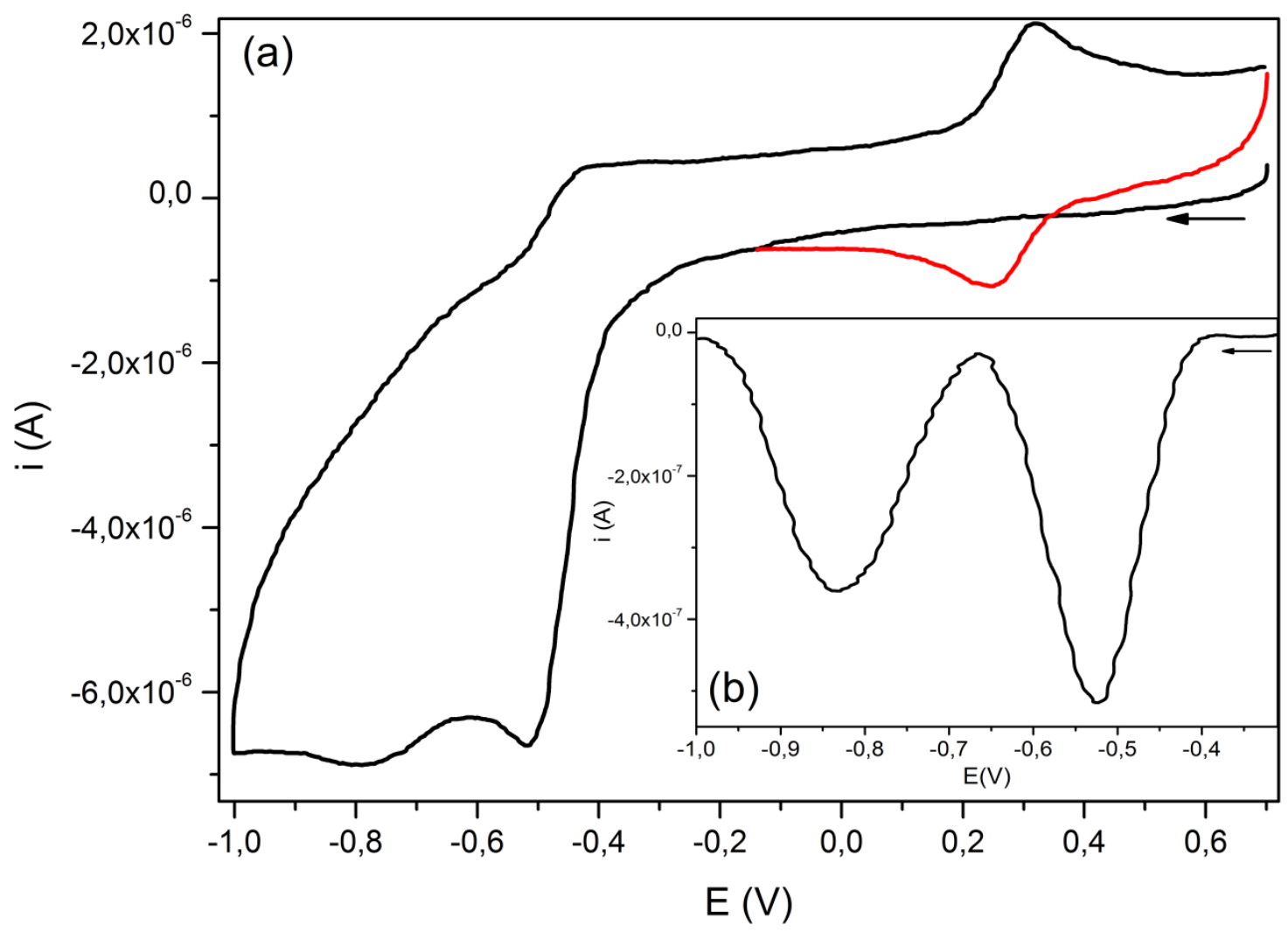

O método eletroquímico de Nicholson e Shain [77] foi utilizado para o cálculo da constante de velocidade de liberação específica de $\mathrm{NO}$ ( $\left.\mathrm{k}_{-\mathrm{NO}}\right)$ do íon trans- $\left[\mathrm{Ru}(\mathrm{NO})\left(\mathrm{NH}_{3}\right)_{4}\left(\mathrm{P}(\mathrm{O})\left(\mathrm{OCH}_{2} \mathrm{CH}_{3}\right)_{2}\right)\right]^{2+}$ em pH 2,0 e $25 \pm 0,1^{\circ} \mathrm{C}$, conforme descrito na Parte Experimental.

A Figura 11 mostra o gráfico de $\mathrm{k} . \tau$ versus $\tau$ para dois valores de potencial de inversão $\left(\mathrm{E}_{\lambda}\right)$ diferentes. Assim, para $\mathrm{o}$ íon 
trans-[Ru(NO) $\left.\left(\mathrm{NH}_{3}\right)_{4} \mathrm{P}(\mathrm{O})\left(\mathrm{OCH}_{2} \mathrm{CH}_{3}\right)_{2}\right]^{2+}$, o valor de $\mathrm{k}_{-\mathrm{NO}}$ é igual a $0,24 \pm 0,01 \mathrm{~s}^{-1}$. Este valor é da mesma ordem de grandeza que o calculado para o trans- $\left[\mathrm{Ru}(\mathrm{NO})\left(\mathrm{NH}_{3}\right)_{4} \mathrm{P}\left(\mathrm{OCH}_{2} \mathrm{CH}_{3}\right)_{3}\right]^{3+}$ $\left(\mathrm{k}_{-\mathrm{NO}}=0,97 \mathrm{~s}^{-1}[51]\right)$, e pode ser considerado um alto valor de velocidade de liberação de NO, principalmente quando comparado ao dos complexos de tetraaminas em que o ligante transposicionado ao grupo NO são N-heterocíclicos $\left(\mathrm{k}_{-\mathrm{NO}}=0,025-0,16 \mathrm{~s}^{-1}[27]\right)$.

A constante de velocidade de liberação específica de $\mathrm{NO}^{-}$(íon nitroxila), k-NO${ }^{-}$, não pode ser calculada por meio do método de Nicholson e Shain [77], pois mesmo utilizado temperatura de $5^{\circ} \mathrm{C}$ e velocidades de varredura de até $2 \mathrm{~V}^{-1}$ o processo de oxidação $\mathrm{NO}^{-} / \mathrm{NO}$ não foi claramente observado. Isto sugere que para o íon trans- $\left[\mathrm{Ru}(\mathrm{NO})\left(\mathrm{NH}_{3}\right)_{4} \mathrm{P}(\mathrm{O})\left(\mathrm{OCH}_{2} \mathrm{CH}_{3}\right)_{2}\right]^{2+} \mathrm{k}_{-\mathrm{NO}}{ }^{-}>\mathrm{k}_{-\mathrm{NO}}$.

Figura 11 - Gráfico de k. $\tau$ versus $\tau$ para $\mathrm{o}$ íon complexo trans- $\left[\mathrm{Ru}(\mathrm{NO})\left(\mathrm{NH}_{3}\right)_{4} \mathrm{P}(\mathrm{O})\left(\mathrm{OCH}_{2} \mathrm{CH}_{3}\right)_{2}\right]^{2+} \mathrm{em} \mathrm{pH} 2,0 ; \mu=0,1 \mathrm{~mol} \mathrm{~L}^{-1} ; \mathrm{C}_{\mathrm{Ru}}=1,5 \times 10^{-3} \mathrm{~mol} \mathrm{~L}^{-}$ ${ }^{1}$ e T $=25 \pm 0,1^{\circ} \mathrm{C}$ (a) Potencial de inversão $\left(\mathrm{E}_{\lambda}\right)=-0,60 \mathrm{~V}$ vs $\mathrm{ECS}$; (b) $\mathrm{E}_{\lambda}=-0,62 \mathrm{~V}$ vs ECS

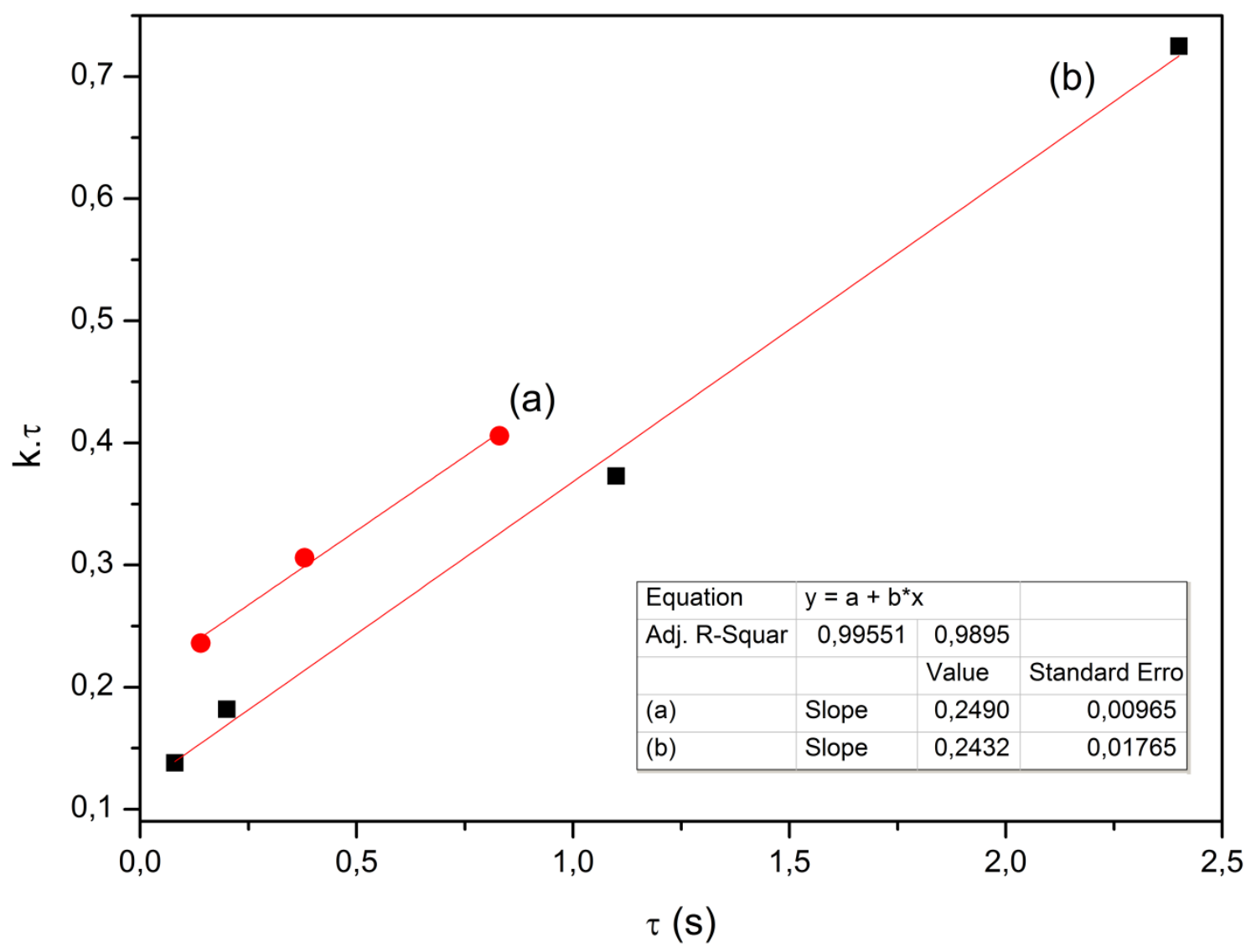




\subsubsection{Espectroscopia Vibracional}

Na Figura 12 é apresentado o espectro vibracional para o complexo trans- $\left[\mathrm{Ru}(\mathrm{NO})\left(\mathrm{NH}_{3}\right)_{4}\left(\mathrm{P}(\mathrm{O})\left(\mathrm{OCH}_{2} \mathrm{CH}_{3}\right)_{2}\right)\right]\left(\mathrm{PF}_{6}\right)_{2} \cdot$ em pastilha de $\mathrm{KBr}$. As principais bandas e respectivas atribuições estão apresentadas na Tabela 5.

Apesar do ligante de dietil fosfito apresentar um próton dissociável, tanto em estado sólido (Figura 12) quanto em solução aquosa (utilizando-se uma variação de concentração hidrogeniônica de 2,0 a $10^{-8} \mathrm{~mol} \mathrm{~L}^{-1}$ ), apenas uma frequência de estiramento para o grupo $\mathrm{NO}^{+}$foi observado em $1887 \mathrm{~cm}^{-1}$. Este comportamento é diferente do observado para os complexos trans- $\left[\mathrm{Ru}(\mathrm{NO})\left(\mathrm{NH}_{3}\right)_{4}\left(\mathrm{P}(\mathrm{OH})_{3}\right)\right]^{3+} /$ trans- $\left[\mathrm{Ru}(\mathrm{NO})\left(\mathrm{NH}_{3}\right)_{4}\left(\mathrm{P}(\mathrm{O})(\mathrm{OH})_{2}\right)\right]^{2+}$ $\left(v \mathrm{NO}^{+}=1892\right.$ e $\left.1879 \mathrm{~cm}^{-1}\right) \quad[59]$ e trans $-\left[\mathrm{Ru}(\mathrm{NO})\left(\mathrm{NH}_{3}\right)_{4}\left(\mathrm{H}_{2} \mathrm{O}\right)\right]^{3+} /$ trans- $\left[\mathrm{Ru}(\mathrm{NO})\left(\mathrm{NH}_{3}\right)_{4}(\mathrm{OH})\right]^{2+}\left(\mathrm{vNO}^{+}=1893\right.$ e $\left.1849 \mathrm{~cm}^{-1}\right)[95]$.

Figura 12 - Espectro na região do infravermelho do composto trans- $\left[\mathrm{Ru}(\mathrm{NO})\left(\mathrm{NH}_{3}\right)_{4}\left(\mathrm{P}(\mathrm{O})\left(\mathrm{OCH}_{2} \mathrm{CH}_{3}\right)_{2}\right)\right]\left(\mathrm{PF}_{6}\right)_{2}$ em pastilha de $\mathrm{KBr}$

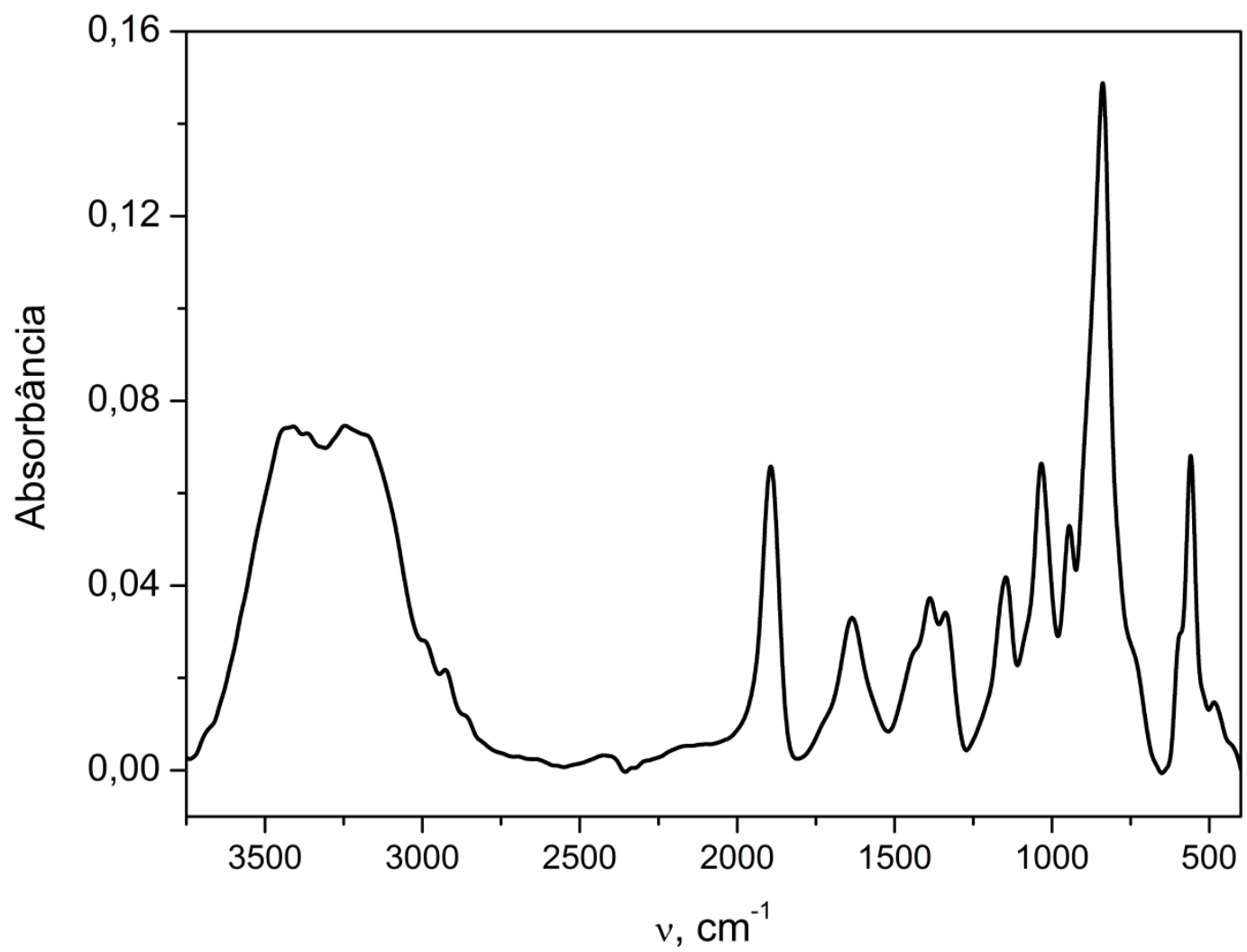


Tabela 5 - Principais bandas e as atribuições propostas

\begin{tabular}{cc}
\hline Número de onda $\left(\mathbf{c m}^{-1}\right)^{*}$ & Atribuições \\
\hline $3428 \mathrm{~m}, 3212 \mathrm{~m}$ & $v_{\mathrm{NH}}, v_{\mathrm{OH}}$ \\
$1887 \mathrm{~F}$ & $v_{\mathrm{NO}}$ \\
$945 \mathrm{~m}$ & $v_{\mathrm{P}-\mathrm{OH}}$ \\
$1153 \mathrm{~m}$ & $v_{\mathrm{P}-\mathrm{O}-\mathrm{H}}$ \\
$1440 \mathrm{~m}$ & $\delta \mathrm{CH}_{3}$ \\
$1390 \mathrm{~m}$ & $\delta_{\mathrm{OCH}_{2}}$ \\
$1631 \mathrm{~m}$ & $\delta_{\mathrm{dNH}}$ \\
$1336 \mathrm{~m}$ & $\delta_{\mathrm{sNH}}$ \\
$554 \mathrm{f}$ & $\delta_{\mathrm{Ru}-\mathrm{NO}}$ \\
$833 \mathrm{~F}$ & $v_{\mathrm{P}(\mathrm{V})-\mathrm{F}}$ \\
* Pastilha de KBr; resolução de $\pm 2 \mathrm{~cm}^{-1} ;$ Intensidades: $\mathrm{F}=$ forte, $\mathrm{m}=$ médio e f $\mathrm{f}=$ fraca.
\end{tabular}

Figura 13 - Espectro de infravermelho: (a) Experimental para o íon trans- $\left[\mathrm{Ru}(\mathrm{NO})\left(\mathrm{NH}_{3}\right)_{4}\left(\mathrm{P}(\mathrm{OH})\left(\mathrm{OCH}_{2} \mathrm{CH}_{3}\right)_{2}\right)\right]^{3+}$ em solução $\mathrm{pH}$ 1,5; (b) Teórico para o íon trans- $\left[\mathrm{Ru}(\mathrm{NO})\left(\mathrm{NH}_{3}\right)_{4}\left(\mathrm{P}(\mathrm{OH})\left(\mathrm{OCH}_{2} \mathrm{CH}_{3}\right)_{2}\right)\right]^{3+}$ e (c) Teórico para 0 íon trans- $\left[\mathrm{Ru}(\mathrm{NO})\left(\mathrm{NH}_{3}\right)_{4}\left(\mathrm{P}(\mathrm{O})\left(\mathrm{OCH}_{2} \mathrm{CH}_{3}\right)_{2}\right)\right]^{2+}$

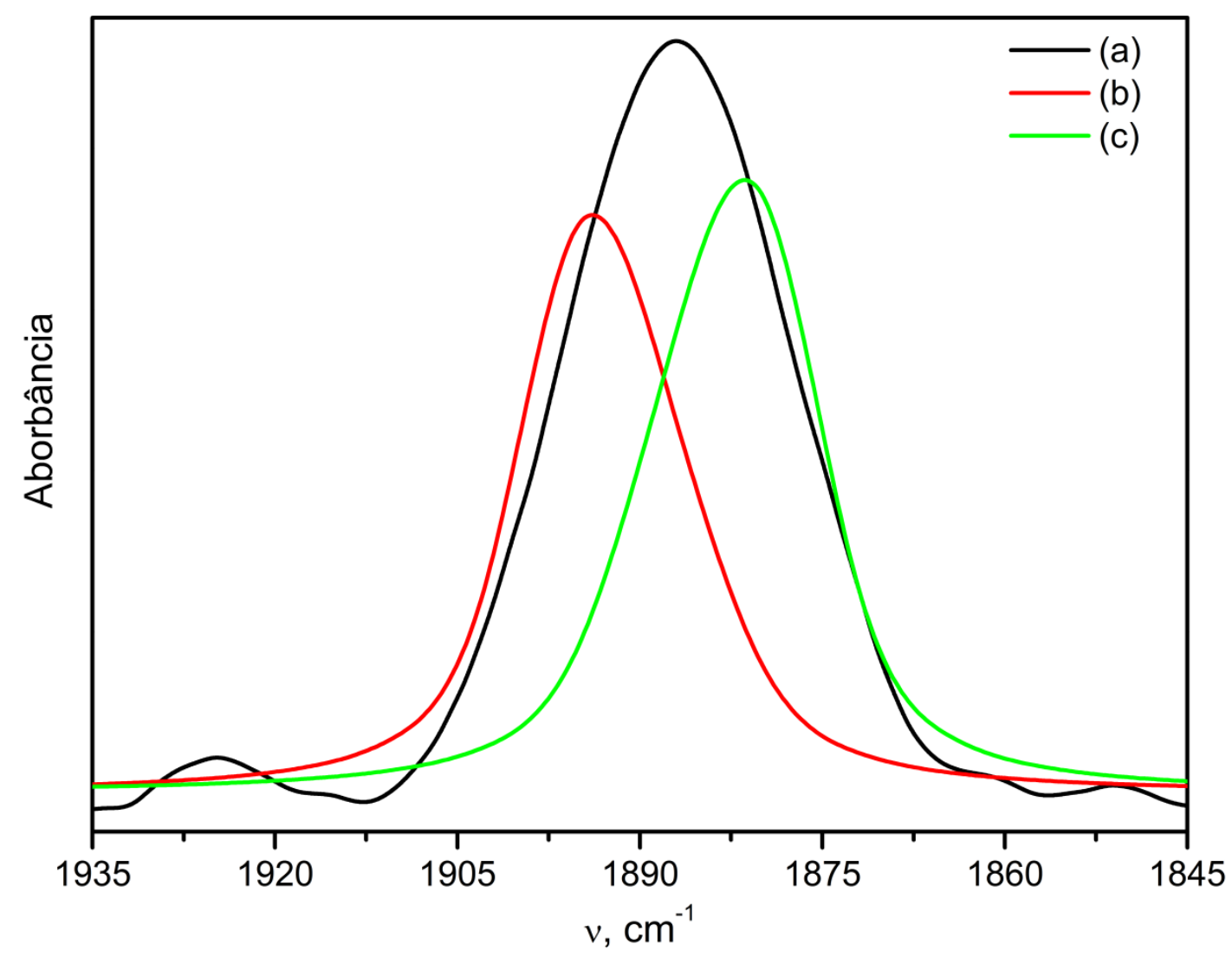




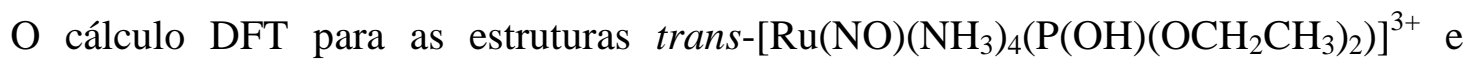
trans- $\left[\mathrm{Ru}(\mathrm{NO})\left(\mathrm{NH}_{3}\right)_{4}\left(\mathrm{P}(\mathrm{O})\left(\mathrm{OCH}_{2} \mathrm{CH}_{3}\right)_{2}\right)\right]^{2+}$ apresentou valores de frequência de estiramento $\mathrm{NO}^{+}$próximos ente si $\left(v_{\mathrm{NO}+}=1893\right.$ e $1883 \mathrm{~cm}^{-1}$, respectivamente $)$, indicando a possibilidade de sobreposição de bandas. A Figura 13 mostra o espectro de infravermelho obtido experimentalmente em $\mathrm{pH}$ 1,5 e os espectros teóricos calculados para as estruturas protonada e desprotonada. É possível observar que o espectro experimental apresenta largura de banda à meia altura $\left(\mathrm{w}_{1 / 2}=22 \mathrm{~cm}^{-1}\right)$ suficiente para conter ambas as frequências de estiramento, o que dificultaria a distinção das mesmas.

\subsubsection{Ressonância Magnética Nuclear}

$\mathrm{O}$ íon trans- $\left[\mathrm{Ru}(\mathrm{NO})\left(\mathrm{NH}_{3}\right)_{4}\left(\mathrm{P}(\mathrm{O})\left(\mathrm{OCH}_{2} \mathrm{CH}_{3}\right)_{2}\right)\right]^{2+}$ foi caracterizado em $\mathrm{D}_{2} \mathrm{O}$ utilizando-se Ressonância Magnética Nuclear de próton $\left({ }^{1} \mathrm{H}\right)$, carbono $\left({ }^{13} \mathrm{C}\right)$ e fósforo $\left({ }^{31} \mathrm{P}\right)$ e os deslocamentos químicos observados encontram-se na Tabela 6.

O espectro de ${ }^{31} \mathrm{P}$ RMN para o íon trans- $\left[\mathrm{Ru}(\mathrm{NO})\left(\mathrm{NH}_{3}\right)_{4}\left(\mathrm{P}(\mathrm{O})\left(\mathrm{OCH}_{2} \mathrm{CH}_{3}\right)_{2}\right)\right]^{2+}$ após 20 minutos em solução pH 1,0 e 7,5 apresentou um único singleto em 68 ppm (Tabela 6). Este comportamento também é diferente do observado para o ligante de ácido fosforoso coordenado ao fragmento $\left[\mathrm{Ru}(\mathrm{NO})\left(\mathrm{NH}_{3}\right)_{4}\right]^{3+}$ que apresenta um singleto em 68 ppm para a sua forma protonada e um singleto em 60 ppm para sua forma desprotonada [59].

Tabela 6 - Deslocamentos químico para o íon trans- $\left[\mathrm{Ru}(\mathrm{NO})\left(\mathrm{NH}_{3}\right)_{4}\left(\mathrm{P}(\mathrm{O})\left(\mathrm{OCH}_{2} \mathrm{CH}_{3}\right)_{2}\right)\right]^{2+}$ em $\mathrm{D}_{2} \mathrm{O}$ (pH 3,0, $\mathrm{CD}_{3} \mathrm{COOD}$ ) utilizando como referências internas o TMSP-D4 e $\mathrm{NH}_{4} \mathrm{PF}_{6}$

\begin{tabular}{ccc}
\hline Núcleo & & $\begin{array}{c}\text { Deslocamento químico } \\
\text { (ppm) }\end{array}$ \\
\hline${ }^{31} \mathrm{P}$ & & 68 \\
${ }^{13} \mathrm{C}$ & $\mathrm{CH}_{2}$ & 64 \\
& $\mathrm{CH}_{3}$ & 18 \\
\multirow{2}{*}{$\mathrm{H}$} & $\mathrm{CH}_{2}$ & 4,14 \\
& $\mathrm{CH}_{3}$ & 1,30 \\
\hline
\end{tabular}


O cálculo das cargas de Mulliken para as estruturas trans- $\left[\mathrm{Ru}(\mathrm{NO})\left(\mathrm{NH}_{3}\right)_{4}\left(\mathrm{P}(\mathrm{OH})\left(\mathrm{OCH}_{2} \mathrm{CH}_{3}\right)_{2}\right)\right]^{3+} \quad \mathrm{e} \quad$ trans- $\left[\mathrm{Ru}(\mathrm{NO})\left(\mathrm{NH}_{3}\right)_{4}\left(\mathrm{P}(\mathrm{O})\left(\mathrm{OCH}_{2} \mathrm{CH}_{3}\right)_{2}\right)\right]^{2+}$ apresentou respectivamente os seguintes valores para a carga sobre o átomo de fósforo: 1,352 e 1,328. Um segundo método para o cálculo da carga atômica (NBO, Natural Bond Order) também foi utilizado, por se tratar de um método mais acurado, apresentando os valores de 2,150 e 2,158 para o átomo de fósforo nas estruturas trans- $\left[\mathrm{Ru}(\mathrm{NO})\left(\mathrm{NH}_{3}\right)_{4}\left(\mathrm{P}(\mathrm{OH})\left(\mathrm{OCH}_{2} \mathrm{CH}_{3}\right)_{2}\right)\right]^{3+} \quad \mathrm{e} \quad$ trans- $\left[\mathrm{Ru}(\mathrm{NO})\left(\mathrm{NH}_{3}\right)_{4}\left(\mathrm{P}(\mathrm{O})\left(\mathrm{OCH}_{2} \mathrm{CH}_{3}\right)_{2}\right)\right]^{2+}$ respectivamente. Ambos os métodos mostram que a alteração na carga dos átomos de fósforo é pequena, portanto os deslocamentos químicos do ${ }^{31} \mathrm{P}$ para as espécies protonada $\mathrm{e}$ desprotonada poderiam apresentar valores muito próximos (dentro da resolução da técnica de $\left.{ }^{31} \mathrm{P} \mathrm{RMN}\right)$. Isto resultaria experimentalmente no aparecimento de apenas um deslocamento químico de ${ }^{31} \mathrm{P}$ em uma ampla faixa de concentração hidrogeniônica.

\subsubsection{Estabilidade do íon trans-[Ru(NO) $\left.\left(\mathrm{NH}_{3}\right)_{4}\left(\mathrm{P}(\mathrm{O})\left(\mathrm{OCH}_{2} \mathrm{CH}_{3}\right)_{2}\right)\right]^{2+}$ em meio} aquoso

O íon trans- $\left[\mathrm{Ru}(\mathrm{NO})\left(\mathrm{NH}_{3}\right)_{4}\left(\mathrm{P}(\mathrm{O})\left(\mathrm{OCH}_{2} \mathrm{CH}_{3}\right)_{2}\right)\right]^{2+}$ foi monitorado em solução aquosa pH 3,0 e $25 \pm 0,5^{\circ} \mathrm{C}$ em função do tempo utilizando ${ }^{31} \mathrm{P}$ RMN. O espectro inicial apresentou apenas um pico em 68 ppm, o qual decai com $\mathrm{k}=8,9 \times 10^{-7} \mathrm{~s}^{-1}\left(\mathrm{t}_{1 / 2}=9\right.$ dias $)$ gerando como produto principal dietil fosfito livre e seus produtos de hidrólise $(15-0 \mathrm{ppm})$ com $\mathrm{k}=9,4 \times 10^{-7} \mathrm{~s}^{-1}\left(\mathrm{t}_{1 / 2}=8,6\right.$ dias $)$, reação 9.

$$
\begin{aligned}
\text { trans }-\left[\mathrm{Ru}(\mathrm{NO})\left(\mathrm{NH}_{3}\right)_{4} \mathrm{P}(\mathrm{O})\left(\mathrm{OCH}_{2} \mathrm{CH}_{3}\right)_{2}\right]^{2+} \underset{\mathrm{pH} 3,0}{\longrightarrow} \text { trans- }\left[\mathrm{Ru}(\mathrm{NO})\left(\mathrm{NH}_{3}\right)_{4}\left(\mathrm{OH}_{2}\right)\right]^{3+}+\mathrm{P}(\mathrm{O})\left(\mathrm{OCH}_{2} \mathrm{CH}_{3}\right)_{2} \\
\delta_{31 \mathrm{P}}=68 \mathrm{ppm}
\end{aligned}
$$

Dois picos de baixa intensidade em 66 e 62 ppm também foram observados e, provavelmente, correspondem às espécies trans- $\left[\mathrm{Ru}(\mathrm{NO})\left(\mathrm{NH}_{3}\right)_{4}\left(\mathrm{P}(\mathrm{OH})_{2}\left(\mathrm{OCH}_{2} \mathrm{CH}_{3}\right)\right)\right]^{3+} \mathrm{e}$ 
trans-[Ru(NO) $\left.\left(\mathrm{NH}_{3}\right)_{4}\left(\mathrm{P}(\mathrm{O})(\mathrm{OH})_{2}\right)\right]^{2+}$, respectivamente. Nestas, as formas hidrolisadas do dietil fosfito (monoetil fosfito e ácido fosforoso) estão coordenadas ao centro metálico. Entretanto, é importante enfatizar que, com base na distribuição de produtos e nos valores calculados de constantes de velocidade, a principal reação do íon trans- $\left[\mathrm{Ru}(\mathrm{NO})\left(\mathrm{NH}_{3}\right)_{4}\left(\mathrm{P}(\mathrm{O})\left(\mathrm{OCH}_{2} \mathrm{CH}_{3}\right)_{2}\right)\right]^{2+}$ em pH 3,0 é a dissociação direta do ligante dietil fosfito (reação 9) e não a sua hidrólise enquanto ligante.

A partir dos dados cinéticos, é possível observar que em $\mathrm{pH} 3,0$ e $25 \pm 0,5^{\circ} \mathrm{C}$ o íon trans- $\left[\mathrm{Ru}(\mathrm{NO})\left(\mathrm{NH}_{3}\right)_{4}\left(\mathrm{P}(\mathrm{O})\left(\mathrm{OCH}_{2} \mathrm{CH}_{3}\right)_{2}\right)\right]^{2+}\left(\mathrm{t}_{1 / 2}=9\right.$ dias $)$ é cerca de 3 vezes mais estável que o trans- $\left[\mathrm{Ru}(\mathrm{NO})\left(\mathrm{NH}_{3}\right)_{4}\left(\mathrm{P}\left(\mathrm{OCH}_{2} \mathrm{CH}_{3}\right)_{3}\right)\right]^{3+}\left(\mathrm{t}_{1 / 2}=2,8\right.$ dias, seção 4.3) e 269 vezes mais estável que o trans- $\left[\mathrm{Ru}(\mathrm{NO})\left(\mathrm{NH}_{3}\right)_{4}\left(\mathrm{P}(\mathrm{O})(\mathrm{OH})_{2}\right)\right]^{2+}\left(\mathrm{t}_{1 / 2}=47,7\right.$ min, seção 4.1) [59].

Entretanto, é importante ressaltar que o comportamento em meio aquoso do íon trans- $\left[\mathrm{Ru}(\mathrm{NO})\left(\mathrm{NH}_{3}\right)_{4}\left(\mathrm{P}(\mathrm{O})\left(\mathrm{OCH}_{2} \mathrm{CH}_{3}\right)_{2}\right)\right]^{2+}$ não é semelhante ao observado para os correspondentes nitrosilo complexos de trietil fosfito e ácido fosforoso [59] nas mesmas condições experimentais. Enquanto o íon trans- $\left[\mathrm{Ru}(\mathrm{NO})\left(\mathrm{NH}_{3}\right)_{4}\left(\mathrm{P}\left(\mathrm{OCH}_{2} \mathrm{CH}_{3}\right)_{3}\right)\right]^{3+}$ sofre hidrólise no ligante de fósforo coordenado e o trans- $\left[\mathrm{Ru}(\mathrm{NO})\left(\mathrm{NH}_{3}\right)_{4}\left(\mathrm{P}(\mathrm{O})(\mathrm{OH})_{2}\right)\right]^{2+}$ isomeriza para o composto coordenado pelo átomo de oxigênio $\left(\right.$ trans $\left.-\left[\mathrm{Ru}(\mathrm{NO})\left(\mathrm{NH}_{3}\right)_{4}\left((\mathrm{O}) \mathrm{P}(\mathrm{OH})_{2}\right)\right]^{2+}\right)$ e, apenas após esta isomerização, o ligante de fósforo é dissociado [59], o dietil fosfito no trans- $\left[\mathrm{Ru}(\mathrm{NO})\left(\mathrm{NH}_{3}\right)_{4}\left(\mathrm{P}(\mathrm{O})\left(\mathrm{OCH}_{2} \mathrm{CH}_{3}\right)_{2}\right)\right]^{2+}$, apesar de apresentar grupamentos etilas passíveis de hidrólise, não é hidrolisado. Ao mesmo tempo, mesmo apresentando um próton ácido $\left(\mathrm{pK}_{\mathrm{a}} \sim 1,5\right)$, não se observa isomerização do dietil fosfito coordenado pelo átomo de fosforo para o coordenado pelo átomo de oxigênio. Entretanto, é observada dissociação direta da molécula de dietil fosfito, sem prévia hidrólise e isomerização. Isto provavelmente ocorre devido ao efeito $\sigma$-indutivo promovido pelos grupos etilas (ausentes no ácido fosforoso) combinado à presença do próton dissociável do grupo hidroxila (ausente no trietil fosfito) que resultam em uma carga negativa adicional sobre o ligante de fósforo. 
Considerando que o trans- $\left[\mathrm{Ru}(\mathrm{NO})\left(\mathrm{NH}_{3}\right)_{4}\left(\mathrm{P}(\mathrm{O})\left(\mathrm{OCH}_{2} \mathrm{CH}_{3}\right)_{2}\right)\right]^{2+}$ é um potencial candidato a doador de $\mathrm{NO} / \mathrm{HNO}$ em meio biológico, a estabilidade deste complexo foi monitorada em função do tempo em pH 7,5 (tampão TRIS, $\mu=0,1 \mathrm{~mol} \mathrm{~L}^{-1}$ ) a $25{ }^{\circ} \mathrm{C}$ por meio de ${ }^{31}$ P RMN, espectroscopia de infravermelho, voltametria cíclica e espectroscopia eletrônica.

A Figura 14 mostra a evolução temporal das áreas dos sinais de ${ }^{31} \mathrm{P}$ RMN para o complexo trans- $\left[\mathrm{Ru}(\mathrm{NO})\left(\mathrm{NH}_{3}\right)_{4}\left(\mathrm{P}(\mathrm{O})\left(\mathrm{OCH}_{2} \mathrm{CH}_{3}\right)_{2}\right)\right]^{2+}$ em pH 7,5 e $25 \pm 0,5^{\circ} \mathrm{C}$. O perfil mostrado na Figura 14 não é trivial, de forma que, possivelmente, reações competitivas e/ou consecutivas estejam ocorrendo.

Figura 14 - Distribuição dos produtos em função do tempo obtido a partir dos dados cinéticos de ${ }^{31} \mathrm{P}$ RMN do íon trans- $\left[\mathrm{Ru}(\mathrm{NO})\left(\mathrm{NH}_{3}\right)_{4}\left(\mathrm{P}(\mathrm{O})\left(\mathrm{OCH}_{2} \mathrm{CH}_{3}\right)_{2}\right)\right]^{2+}$ em pH 7,5 e $25 \pm 0,5^{\circ} \mathrm{C}$;

(a) $\delta_{31 \mathrm{P}}=68 \mathrm{ppm}$; trans $-\left[\mathrm{Ru}(\mathrm{NO})\left(\mathrm{NH}_{3}\right)_{4}\left(\mathrm{P}(\mathrm{O})\left(\mathrm{OCH}_{2} \mathrm{CH}_{3}\right)_{2}\right)\right]^{2+}$;

(b) $\delta_{31 \mathrm{P}}=138 \mathrm{ppm}$; trans- $\left[\mathrm{Ru}\left(\mathrm{H}_{2} \mathrm{O}\right)\left(\mathrm{NH}_{3}\right)_{4}\left(\mathrm{P}(\mathrm{O})\left(\mathrm{OCH}_{2} \mathrm{CH}_{3}\right)_{2}\right)\right]^{+}$;

(c) $\delta_{31 \mathrm{P}}=136$ ppm; trans- $\left[\mathrm{Ru}\left(\mathrm{H}_{2} \mathrm{O}\right)\left(\mathrm{NH}_{3}\right)_{4}\left(\mathrm{P}(\mathrm{O})(\mathrm{OH})\left(\mathrm{OCH}_{2} \mathrm{CH}_{3}\right)\right)\right]^{+}$;

(d) $\delta_{31 \mathrm{P}}=0-15 \mathrm{ppm} ; \mathrm{P}(\mathrm{OH})\left(\mathrm{OCH}_{2} \mathrm{CH}_{3}\right)_{2}$ e espécies hidrolisadas

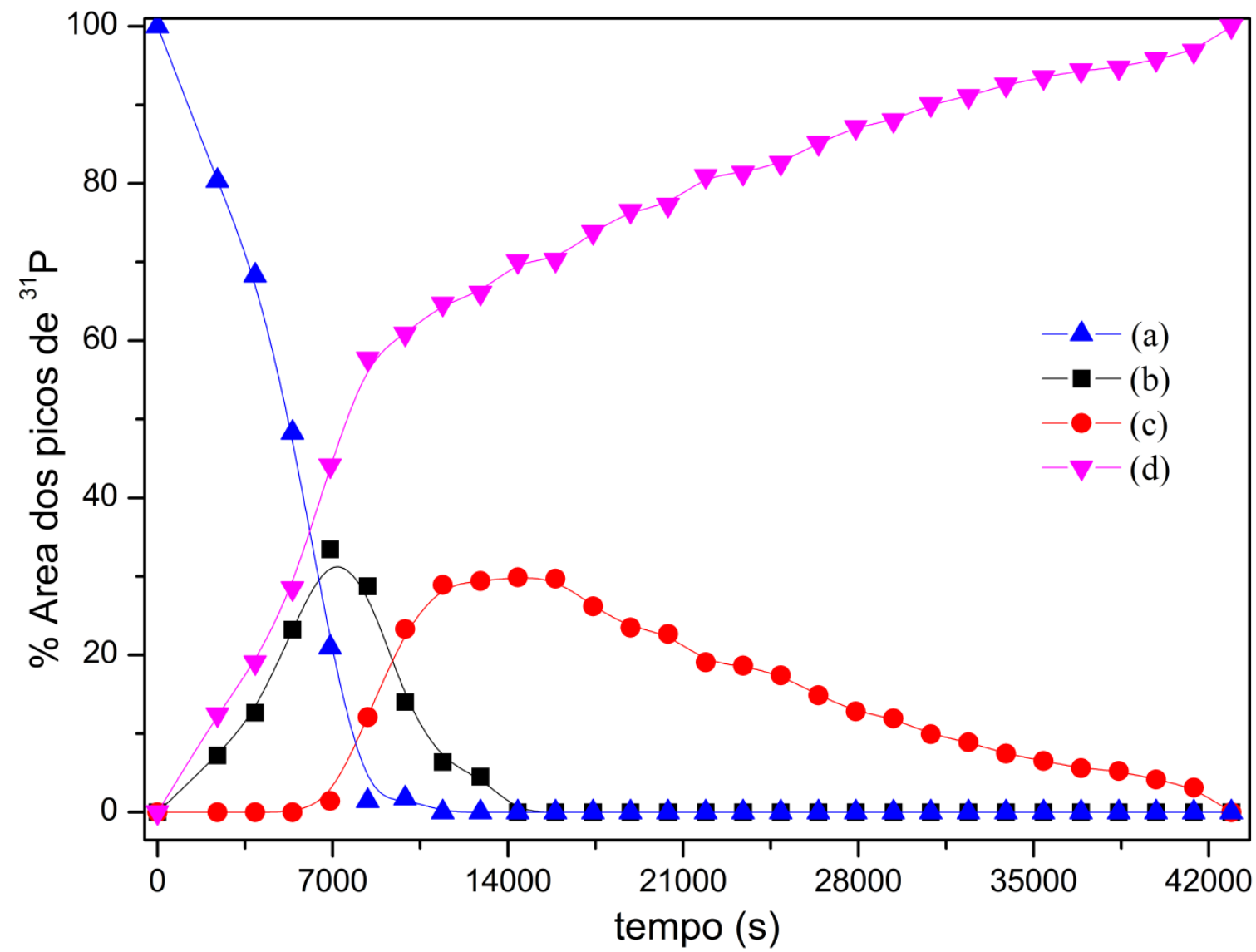


A fim de entender o comportamento químico em solução aquosa $\mathrm{pH} 7,5$ do íon trans- $\left[\mathrm{Ru}(\mathrm{NO})\left(\mathrm{NH}_{3}\right)_{4} \mathrm{P}(\mathrm{O})\left(\mathrm{OCH}_{2} \mathrm{CH}_{3}\right)_{2}\right]^{2+}$, a formação e o decaimento dos picos de ${ }^{31} \mathrm{P}$ foram tratados admitindo um comportamento cinético de primeira ordem, e obtendo-se a constante de velocidade observada $(k)$ por meio da curva de $\ln \left(A-A_{t}\right)$ por tempo.

Na Figura 14 é possível observar que, no intervalo de tempo de 0-6.000 segundos, o pico em $68 \mathrm{ppm}$ decresce originando picos de fósforo livre $\left(\mathrm{k}_{\mathrm{f}(15-0 \mathrm{ppm}) \text { total }}=1,14 \times 10^{-4} \mathrm{~s}^{-1}\right) \mathrm{e}$ um pico em $138 \mathrm{ppm}\left(\mathrm{k}_{\mathrm{f}(138 \mathrm{ppm})}=7,50 \times 10^{-5} \mathrm{~s}^{-1}\right)$.

Após 6.000 segundos o pico em 138 ppm decai formando quantitativamente o pico em $136 \mathrm{ppm}\left(\mathrm{k}_{\mathrm{f}(136 \mathrm{ppm})}=7,45 \times 10^{-5} \mathrm{~s}^{-1}\right)$, o qual, decai em seguida formando quantitativamente fósforo livre $\left(\mathrm{k}_{\mathrm{d}(136 \mathrm{ppm})}=\mathrm{k}_{\mathrm{f}(15-0 \mathrm{ppm})}=6,25 \times 10^{-5} \mathrm{~s}^{-1}\right)$.

O fósforo livre originado em solução tem como uma de suas fontes a espécie em 136 ppm. Como a formação de fósforo livre no período de 0-6.000 segundos ocorre com $\mathrm{k}_{\mathrm{f}(15-0 \mathrm{ppm}) t \mathrm{tatal}}=1,14 \times 10^{-4} \mathrm{~s}^{-1}$ e a espécie cujo sinal ocorre em $136 \mathrm{ppm}$ gera fósforo livre com $\mathrm{k}_{\mathrm{f}(15-0 \mathrm{ppm}) 1}=6,25 \times 10^{-5} \mathrm{~s}^{-1}$, é possível uma segunda reação que origine fósforo livre em solução com $\mathrm{k}_{\mathrm{f}(15-0 \mathrm{ppm}) 2}=5,15 \times 10^{-5} \mathrm{~s}^{-1}\left(\mathrm{k}_{\mathrm{f}(15-0 \mathrm{ppm}) 2}=\mathrm{k}_{\mathrm{f}(15-0 \mathrm{ppm}) \text { total }}-\mathrm{k}_{\mathrm{f}(15-0 \mathrm{ppm}) 1}\right)$. Isto sugere que também possa ocorrer a dissociação direta do ligante dietil fosfito a partir do nitrosilo complexo (reação 10) em pH 7,5, a semelhança do observado em pH 3,0. Assim, o pico em 68 ppm decairia formando como produtos o pico em 138 ppm e também os picos de fósforo livre entre $15-0 \mathrm{ppm}$.

A fim de identificar a espécies que estão sendo formadas, e admitindo a possibilidade da formação da espécie trans- $\left[\mathrm{Ru}\left(\mathrm{H}_{2} \mathrm{O}\right)\left(\mathrm{NH}_{3}\right)_{4}\left(\mathrm{P}(\mathrm{O})\left(\mathrm{OCH}_{2} \mathrm{CH}_{3}\right)_{2}\right)\right]^{+}, \quad$ o complexo trans- $\left[\mathrm{Ru}\left(\mathrm{H}_{2} \mathrm{O}\right)\left(\mathrm{NH}_{3}\right)_{4}\left(\mathrm{P}(\mathrm{O})\left(\mathrm{OCH}_{2} \mathrm{CH}_{3}\right)_{2}\right)\right]\left(\mathrm{PF}_{6}\right)$ foi sintetizado. O espectro de ${ }^{31} \mathrm{P} \mathrm{RMN}$ deste íon em pH 7,5 mostrou um pico em 138 ppm, que apresenta decréscimo de sua área em função do tempo, formando um pico em 136 ppm. Assim, o pico em 138 ppm foi atribuído a espécie trans- $\left[\mathrm{Ru}\left(\mathrm{H}_{2} \mathrm{O}\right)\left(\mathrm{NH}_{3}\right)_{4}\left(\mathrm{P}(\mathrm{O})\left(\mathrm{OCH}_{2} \mathrm{CH}_{3}\right)_{2}\right)\right]^{+}$e o pico em $136 \mathrm{ppm}$ ao produto da 
hidrólise do complexo de dietil fosfito: trans-[Ru( $\left.\left.\mathrm{H}_{2} \mathrm{O}\right)\left(\mathrm{NH}_{3}\right)_{4}\left(\mathrm{P}(\mathrm{O})(\mathrm{OH})\left(\mathrm{OCH}_{2} \mathrm{CH}_{3}\right)\right)\right]^{+}$, reação 13. Após a formação do trans- $\left[\mathrm{Ru}\left(\mathrm{H}_{2} \mathrm{O}\right)\left(\mathrm{NH}_{3}\right)_{4}\left(\mathrm{P}(\mathrm{O})(\mathrm{OH})\left(\mathrm{OCH}_{2} \mathrm{CH}_{3}\right)\right)\right]^{+}$, o monoetil fosfito é dissociado ocorrendo a formação do íon trans- $\left[\mathrm{Ru}\left(\mathrm{OH}_{2}\right)_{2}\left(\mathrm{NH}_{3}\right)_{4}\right]^{2+}$ e fósforo livre em solução (reação 14).

Dessa forma, uma sequência destas reações pode ser esquematizada da seguinte forma:

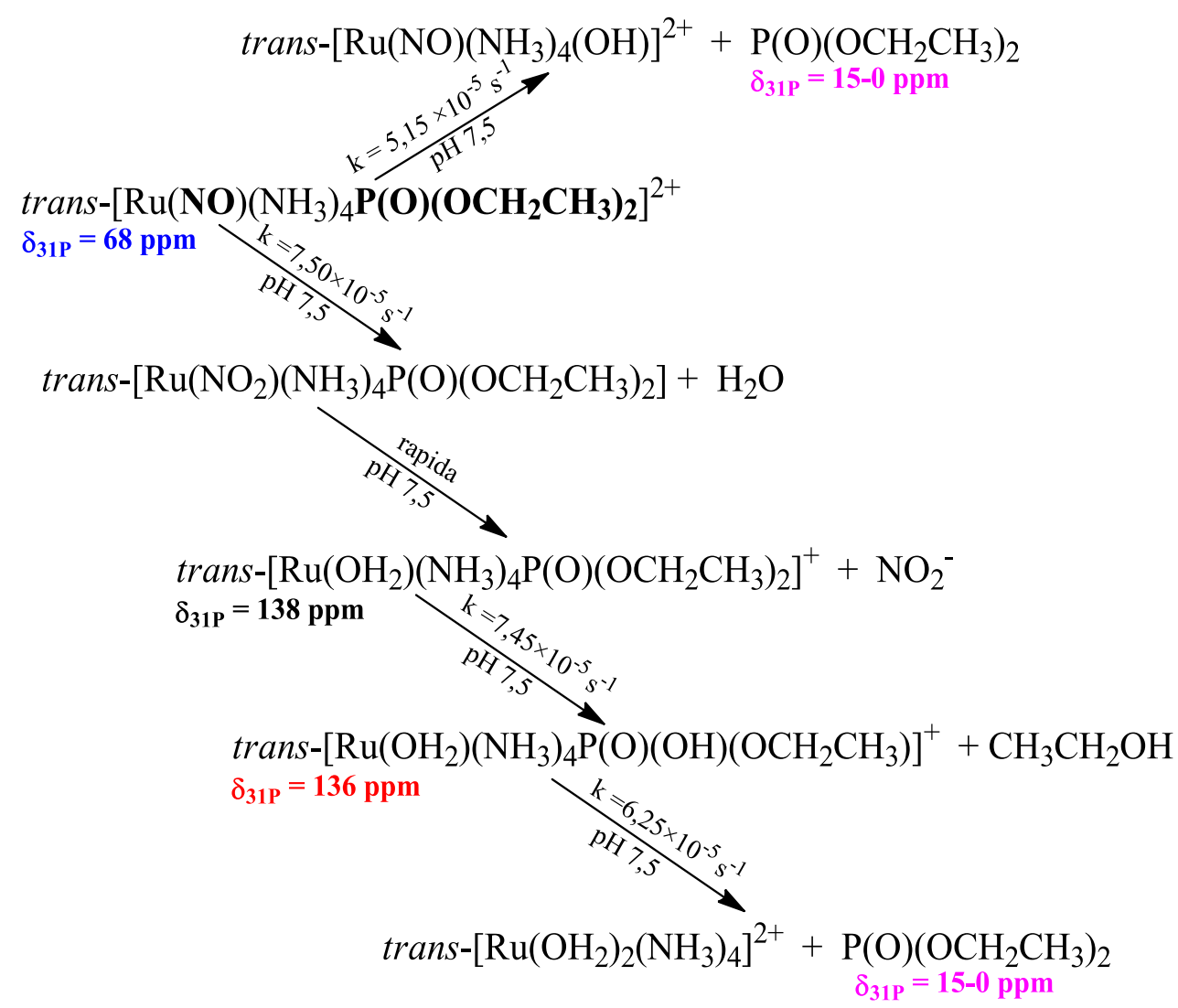

Para a formação da espécie trans- $\left[\mathrm{Ru}\left(\mathrm{H}_{2} \mathrm{O}\right)\left(\mathrm{NH}_{3}\right)_{4} \mathrm{P}(\mathrm{O})\left(\mathrm{OCH}_{2} \mathrm{CH}_{3}\right)_{2}\right]^{+}$em 138 ppm a partir do trans-[Ru(NO) $\left.\left(\mathrm{NH}_{3}\right)_{4} \mathrm{P}(\mathrm{O})\left(\mathrm{OCH}_{2} \mathrm{CH}_{3}\right)_{2}\right]^{2+}$ é necessário que ocorra uma reação centrada no ligante nitrosônio. Segundo a literatura [51; 101], nitrosilos complexos sofrem ataque nucleofílico no grupo $\mathrm{NO}^{+} \mathrm{em} \mathrm{pH} \geq 5$ dando origem a nitrito complexos. A formação de nitrito complexos do tipo trans- $\left[\mathrm{Ru}\left(\mathrm{NO}_{2}\right)\left(\mathrm{NH}_{3}\right)_{4} \mathrm{P}(\mathrm{III})\right]^{\mathrm{n}}$ pode ser identificada de forma 
indireta, por espectrofotometria eletrônica, utilizando pirazina como ligante auxiliar, de acordo com as reações 15 e 16 [101].

$$
\begin{aligned}
& \text { trans- }\left[\mathrm{Ru}\left(\mathrm{NO}_{2}\right)\left(\mathrm{NH}_{3}\right)_{4} \mathrm{P}(\mathrm{III})\right]^{\mathrm{n}}+\mathrm{H}_{2} \mathrm{O} \stackrel{\text { lenta }}{\rightleftharpoons} \text { trans- }\left[\mathrm{Ru}\left(\mathrm{H}_{2} \mathrm{O}\right)\left(\mathrm{NH}_{3}\right)_{4} \mathrm{P}(\mathrm{III})_{2}\right]^{\mathrm{n}+1}+\mathrm{NO}_{2}^{-} \\
& \text {trans }-\left[\mathrm{Ru}\left(\mathrm{H}_{2} \mathrm{O}\right)\left(\mathrm{NH}_{3}\right)_{4} \mathrm{P}(\mathrm{III})\right]^{\mathrm{n}+1}+\mathrm{pz} \stackrel{\text { rápida }}{\rightleftharpoons} \text { trans- }\left[\mathrm{Ru}(\mathrm{pz})\left(\mathrm{NH}_{3}\right)_{4} \mathrm{P}(\mathrm{III})_{2}\right]^{\mathrm{n}+1}+\mathrm{H}_{2} \mathrm{O}
\end{aligned}
$$

Admitindo esta possibilidade, uma solução $\mathrm{pH} \quad 7,5$ contendo trans- $\left[\mathrm{Ru}(\mathrm{NO})\left(\mathrm{NH}_{3}\right)_{4}\left(\mathrm{P}(\mathrm{O})\left(\mathrm{OCH}_{2} \mathrm{CH}_{3}\right)_{2}\right)\right]^{2+}$ e excesso de pirazina a $25 \pm 0,1^{\circ} \mathrm{C}$ foi monitorada por espectroscopia eletrônica observando-se a formação do complexo de pirazina $(\lambda=400 \mathrm{~nm}$ [56]). A Figura 15 mostra a formação da banda em $400 \mathrm{~nm}$ com uma constante de velocidade observada para a aquação do ligante $\mathrm{NO}_{2}^{-}$no complexo trans- $\left[\mathrm{Ru}(\mathrm{NO})\left(\mathrm{NH}_{3}\right)_{4}\left(\mathrm{P}(\mathrm{O})\left(\mathrm{OCH}_{2} \mathrm{CH}_{3}\right)_{2}\right)\right]^{2+}$ igual a $1,19 \times 10^{-4} \mathrm{~s}^{-1}$.

A solução $\mathrm{pH} 7,5$ contendo trans- $\left[\mathrm{Ru}(\mathrm{NO})\left(\mathrm{NH}_{3}\right)_{4}\left(\mathrm{P}(\mathrm{O})\left(\mathrm{OCH}_{2} \mathrm{CH}_{3}\right)_{2}\right)\right]^{2+}$ e excesso de pirazina a $25 \pm 0,1^{\circ} \mathrm{C}$ também foi monitorada por ${ }^{31} \mathrm{P}$ RMN. Neste caso, o nitrosilo complexo de dietil fosfito $\left(\delta_{31 \mathrm{P}}=68 \mathrm{ppm}\right)$ decai formando fósforo livre e um pico em $132 \mathrm{ppm}$ referente ao íon trans- $\left[\mathrm{Ru}(\mathrm{pz})\left(\mathrm{NH}_{3}\right)_{4}\left(\mathrm{P}(\mathrm{O})\left(\mathrm{OCH}_{2} \mathrm{CH}_{3}\right)_{2}\right)\right]^{+}$. Esta última espécie foi identificada por meio de ${ }^{31} \mathrm{P}$ RMN da solução $\mathrm{pH} 7,5$ contendo $1 \mathrm{M}$ de pirazina e o íon complexo trans- $\left[\mathrm{Ru}\left(\mathrm{H}_{2} \mathrm{O}\right)\left(\mathrm{NH}_{3}\right)_{4}\left(\mathrm{P}(\mathrm{O})\left(\mathrm{OCH}_{2} \mathrm{CH}_{3}\right)_{2}\right)\right]^{+}$. A formação do pico em 132 ppm ocorreu com $\mathrm{k}_{\mathrm{f}(132 \mathrm{ppm})}=1,18 \times 10^{-4} \mathrm{~s}^{-1}$, este valor coincide com o valor calculado utilizando espectroscopia eletrônica $\left(\mathrm{k}=1,19 \times 10^{-4} \mathrm{~s}^{-1}\right)$.

Dessa forma, é razoável propor que a velocidade de aquação dos íons $\mathrm{NO}_{2}^{-}$no trans-[Ru(NO $\left.\left.\mathrm{NO}_{2}\right)\left(\mathrm{NH}_{3}\right)_{4}\left(\mathrm{P}(\mathrm{O})\left(\mathrm{OCH}_{2} \mathrm{CH}_{3}\right)_{2}\right)\right]$ é superior a velocidade de ataque nucleofílico sobre o grupo $\mathrm{NO}^{+}$no íon complexo trans-[Ru(NO)( $\left.\left.\mathrm{NH}_{3}\right)_{4}\left(\mathrm{P}(\mathrm{O})\left(\mathrm{OCH}_{2} \mathrm{CH}_{3}\right)_{2}\right)\right]^{2+}$, o que impede o acúmulo da espécie trans-[Ru( $\left.\left(\mathrm{NO}_{2}\right)\left(\mathrm{NH}_{3}\right)_{4}\left(\mathrm{P}(\mathrm{O})\left(\mathrm{OCH}_{2} \mathrm{CH}_{3}\right)_{2}\right)\right]$ em solução e impossibilita a visualização de seu deslocamento químico de ${ }^{31} \mathrm{P}$, reações 11 e 12 . 
Figura 15 - Espectro de UV-vis para o íon trans- $\left[\mathrm{Ru}(\mathrm{NO})\left(\mathrm{NH}_{3}\right)_{4}\left(\mathrm{P}(\mathrm{O})\left(\mathrm{OCH}_{2} \mathrm{CH}_{3}\right)_{2}\right)\right]^{2+}$ na presença de excesso de pirazina em $\mathrm{pH} 7,5$ e $25 \pm 0,1^{\circ} \mathrm{C}$. Inserção: Curva de $\ln \left(\mathrm{A}-\mathrm{A}_{\mathrm{t}}\right)$ versus tempo (s)

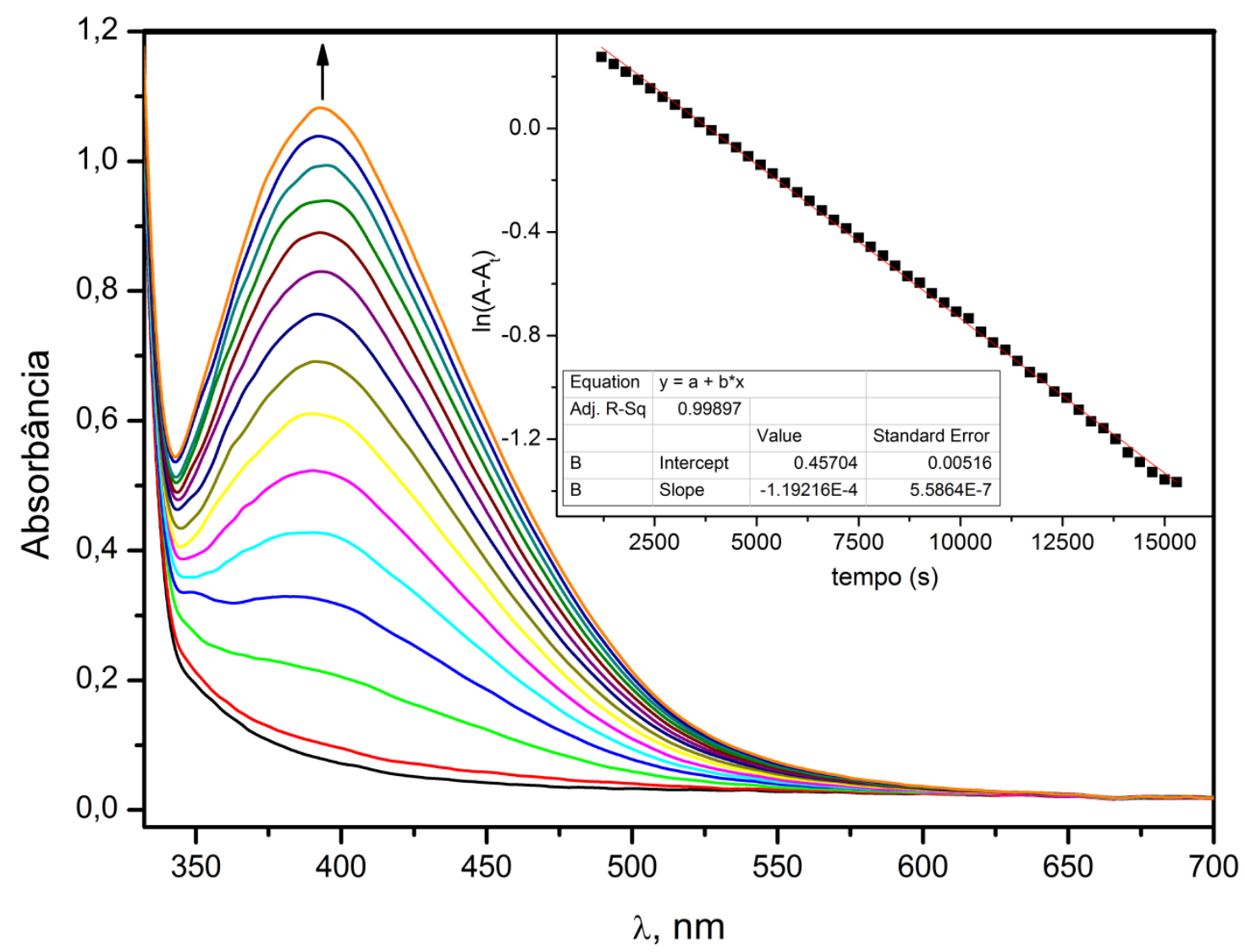

A variação do espectro de infravermelho da solução $\mathrm{pH}$ 7,5 contendo o íon trans- $\left[\mathrm{Ru}(\mathrm{NO})\left(\mathrm{NH}_{3}\right)_{4}\left(\mathrm{P}(\mathrm{O})\left(\mathrm{OCH}_{2} \mathrm{CH}_{3}\right)_{2}\right)\right]^{2+} \quad($ Figura 16$)$ mostra um decréscimo da $v_{\mathrm{NO}+}=1887 \mathrm{~cm}^{-1}$ em função do tempo, o que é consistente com o consumo do nitrosilo complexo de dietil fosfito neste meio. Entretanto, não se observa a formação da $v_{\mathrm{NO}+}=1850$ $\mathrm{cm}^{-1}$ referente ao íon trans-[Ru(NO)(NH$\left.)_{4}(\mathrm{OH})\right]^{2+}$ o que, provavelmente, ocorre devido à baixa sensibilidade desta técnica em solução aquosa. 
Figura 16 - Espectro de infravermelho para o trans- $\left[\mathrm{Ru}(\mathrm{NO})\left(\mathrm{NH}_{3}\right)_{4}\left(\mathrm{P}(\mathrm{O})\left(\mathrm{OCH}_{2} \mathrm{CH}_{3}\right)_{2}\right)\right]^{2+} \mathrm{em}$ pH 7,5 (tampão TRIS na concentração de $0,20 \mathrm{~mol} \mathrm{~L}^{-1}$ ), $\mathrm{C}_{\mathrm{Ru}}=7,8 \times 10^{-2} \mathrm{~mol} \mathrm{~L}^{-1}$ e espaçador de $0,05 \mathrm{~mm}$

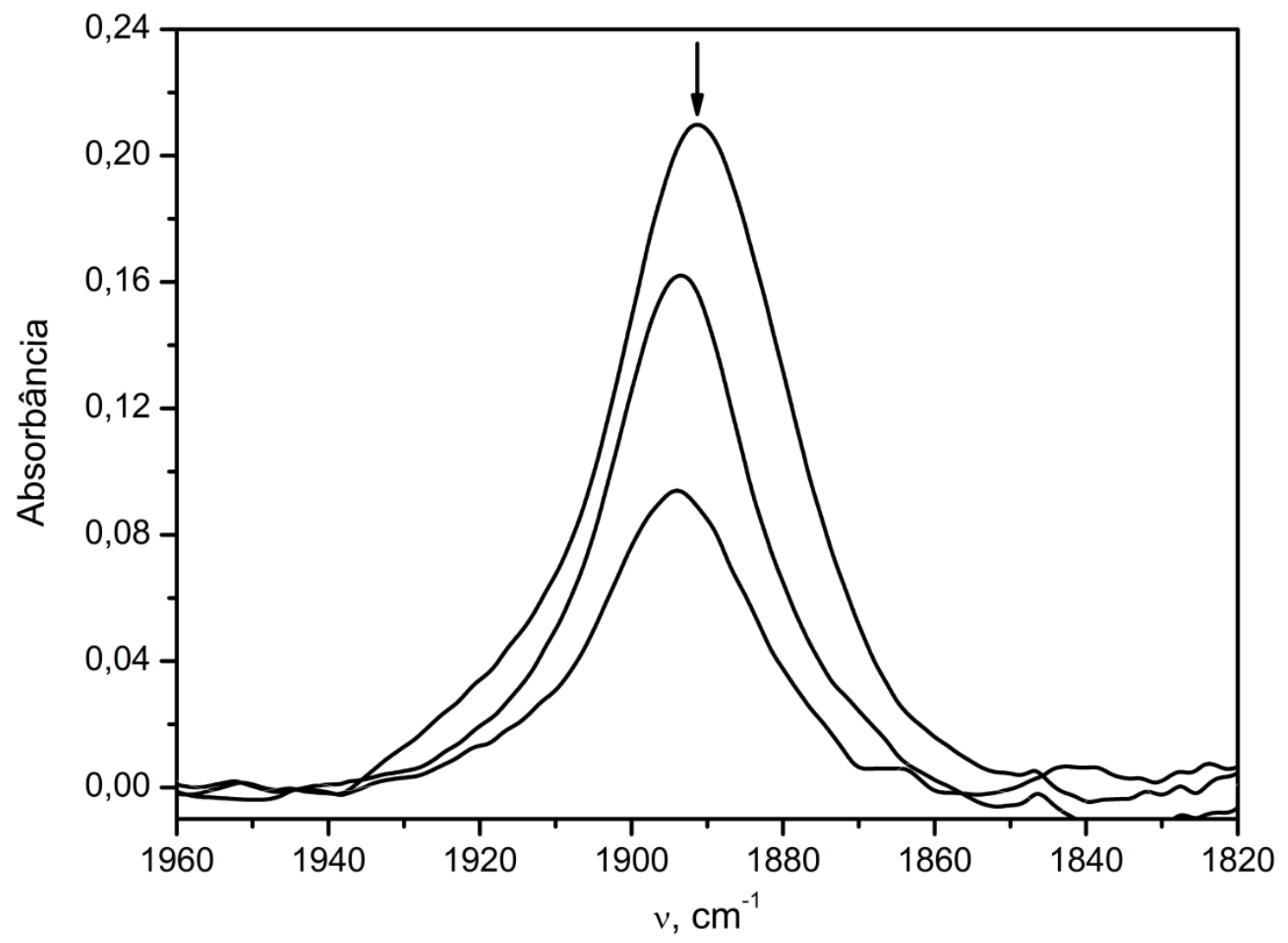

O voltamograma cíclico do trans- $\left[\mathrm{Ru}(\mathrm{NO})\left(\mathrm{NH}_{3}\right)_{4}\left(\mathrm{P}(\mathrm{O})\left(\mathrm{OCH}_{2} \mathrm{CH}_{3}\right)_{2}\right)\right]^{2+}$ em pH 7,5 e $25 \pm 0,1^{\circ} \mathrm{C}$ apresentou um processo reversível referente ao par $\mathrm{Ru}(\mathrm{III}) / \mathrm{Ru}(\mathrm{II})$ em $0,15 \mathrm{~V}$ e os processos de redução dos fragmentos $\mathrm{NO}^{+} / \mathrm{NO}$ e $\mathrm{NO} / \mathrm{NO}^{-}(\mathrm{E}=-0,50$ e $-0,80 \mathrm{~V}$ vs ECS $)$, o que mostra que este nitrosilo complexo é passível de ser reduzido por redutores biológicos em pH 7,5 (Figura 17). Em função do tempo, a corrente destes processos diminui indicando o consumo do nitrosilo complexo.

É interessante notar que o par $\mathrm{Ru}(\mathrm{III}) / \mathrm{Ru}(\mathrm{II})$ nas espécies trans- $\left[\mathrm{Ru}\left(\mathrm{H}_{2} \mathrm{O}\right)\left(\mathrm{NH}_{3}\right)_{4}\left(\mathrm{P}(\mathrm{O})\left(\mathrm{OCH}_{2} \mathrm{CH}_{3}\right)_{2}\right)\right]^{2+} \quad / \quad$ trans- $\left[\mathrm{Ru}\left(\mathrm{H}_{2} \mathrm{O}\right)\left(\mathrm{NH}_{3}\right)_{4}\left(\mathrm{P}(\mathrm{O})\left(\mathrm{OCH}_{2} \mathrm{CH}_{3}\right)_{2}\right)\right]^{+}$ sofre um deslocamento de $130 \mathrm{mV}$ quando os experimentos são realizados a pH 1,0 e pH 7,5, provavelmente devido à desprotonação do ligante dietil fosfito. 
Figura 17 - (a) Voltamograma cíclico para o íon trans- $\left[\mathrm{Ru}(\mathrm{NO})\left(\mathrm{NH}_{3}\right)_{4}\left(\mathrm{P}(\mathrm{O})\left(\mathrm{OCH}_{2} \mathrm{CH}_{3}\right)_{2}\right)\right]^{2+}$ em solução pH 7,5, $\mu=0,1 \mathrm{~mol} \mathrm{~L}^{-1}, \mathrm{C}_{\mathrm{Ru}}=1,5 \times 10^{-3} \mathrm{~mol} \mathrm{~L}^{-1}, \mathrm{~T}=25 \pm 0,1{ }^{\circ} \mathrm{C}$, velocidade $=$ $100 \mathrm{mV} \mathrm{s}^{-1}$; (b) Voltamograma de pulso diferencial realizado em velocidade de $20 \mathrm{mV} \mathrm{s}^{-1} \mathrm{e} \mathrm{T}$ $=5 \pm 0,1^{\circ} \mathrm{C}$

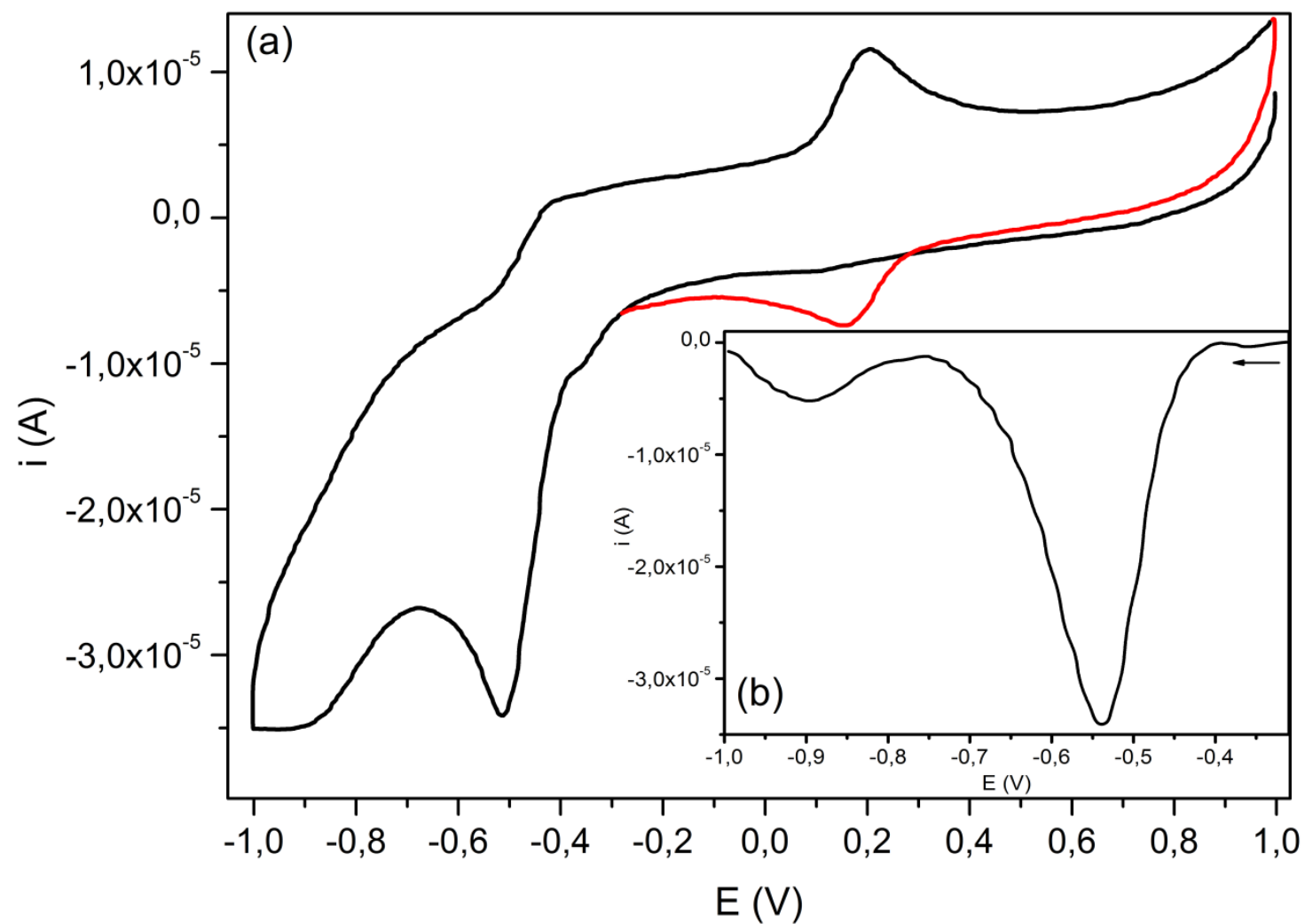

Os dados apresentados sugerem que o íon trans- $\left[\mathrm{Ru}(\mathrm{NO})\left(\mathrm{NH}_{3}\right)_{4}\left(\mathrm{P}(\mathrm{O})\left(\mathrm{OCH}_{2} \mathrm{CH}_{3}\right)_{2}\right)\right]^{2+}$ é mais estável com relação ao ataque nucleofílico tanto no fosfito quanto no nitrosônio do que os outros nitrosilos complexos da série [59; 101]. Em solução pH 7,5 e $25^{\circ} \mathrm{C}$ o íon trans- $\left[\mathrm{Ru}(\mathrm{NO})\left(\mathrm{NH}_{3}\right)_{4}\left(\mathrm{P}(\mathrm{O})\left(\mathrm{OCH}_{2} \mathrm{CH}_{3}\right)_{2}\right)\right]^{2+}$ apresenta $\mathrm{t}_{1 / 2}=1,5 \mathrm{~h}$, sendo consumido de forma competitiva por meio da dissociação do dietil fosfito e do ataque nucleofílico sobre o grupamento nitrosônio.

A formação do produto trans- $\left[\mathrm{Ru}(\mathrm{pz})\left(\mathrm{NH}_{3}\right)_{4}\left(\mathrm{P}(\mathrm{O})\left(\mathrm{OCH}_{2} \mathrm{CH}_{3}\right)_{2}\right)\right]^{+}$a partir do trans-[Ru(NO) $\left.\left(\mathrm{NH}_{3}\right)_{4}\left(\mathrm{P}(\mathrm{O})\left(\mathrm{OCH}_{2} \mathrm{CH}_{3}\right)_{2}\right)\right]^{2+}$ mostra que a aquação do ligante nitrito no complexo trans-[Ru(NO 2$\left.)\left(\mathrm{NH}_{3}\right)_{4}\left(\mathrm{P}(\mathrm{O})\left(\mathrm{OCH}_{2} \mathrm{CH}_{3}\right)_{2}\right)\right]$ em pH 7,5 $\left(1,19 \times 10^{-4} \mathrm{~s}^{-1}\right)$ é cerca de 100 vezes mais lenta do que no íon complexo trans- $\left[\mathrm{Ru}\left(\mathrm{NO}_{2}\right)\left(\mathrm{NH}_{3}\right)_{4}\left(\mathrm{P}\left(\mathrm{OCH}_{2} \mathrm{CH}_{3}\right)_{3}\right)\right]^{+}$ 
$\left(1,30 \times 10^{-2} \mathrm{~s}^{-1}\right)$ [76]. Este ganho de estabilidade do nitrito complexo, em relação à aquação dos íons nitrito, favorece o ciclo catalítico in vivo (Figura 18), no qual o nitrito complexo se converte em nitrosilo complexo, reação que ocorre de forma quantitativa dependendo do pH do meio [101], e reconstitui o doador de NO/HNO em meio fisiológico. Isto torna o íon trans-[Ru(NO) $\left.\left(\mathrm{NH}_{3}\right)_{4}\left(\mathrm{P}(\mathrm{O})\left(\mathrm{OCH}_{2} \mathrm{CH}_{3}\right)_{2}\right)\right]^{2+}$ um importante candidato a testes biológicos.

Figura 18 - Ciclo catalítico para a conversão de nitrito a óxido nítrico por meio de complexos de rutênio

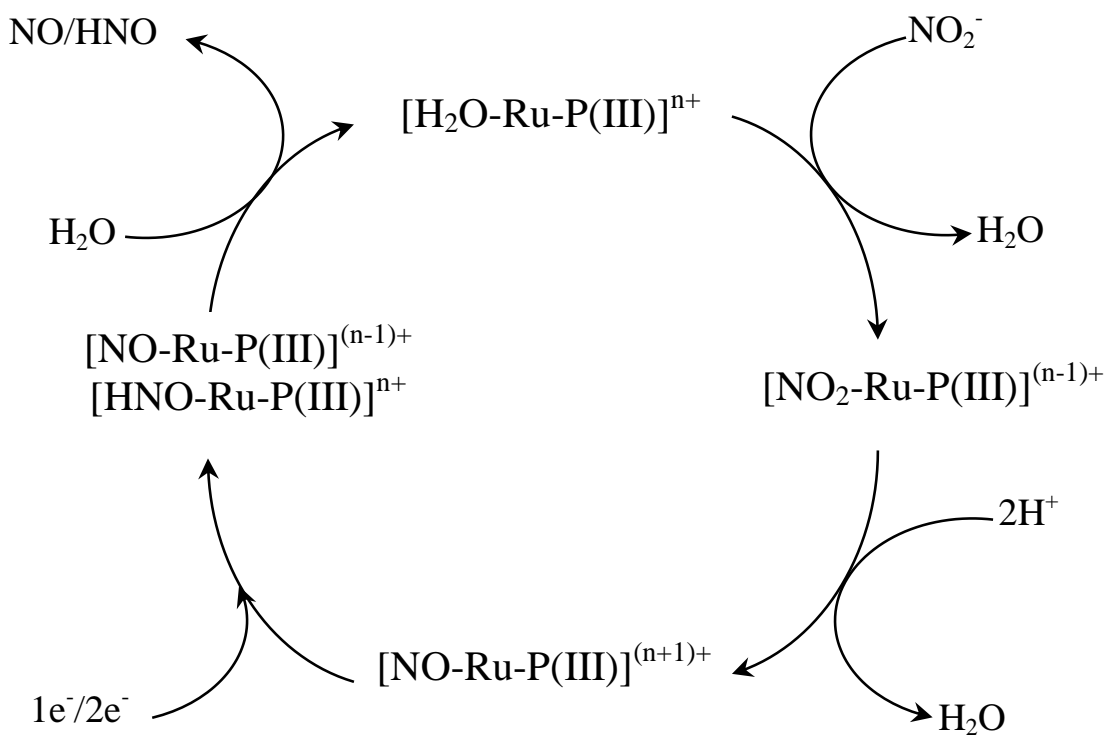

Testes in vitro e in vivo com o trans- $\left[\mathrm{Ru}(\mathrm{NO})\left(\mathrm{NH}_{3}\right)_{4}\left(\mathrm{P}(\mathrm{O})\left(\mathrm{OCH}_{2} \mathrm{CH}_{3}\right)_{2}\right)\right]^{2+}$ estão sendo realizados. Resultados preliminares relacionados a efeitos anti-inflamatórios indicam que a dose de $100 \mu$ mol $\mathrm{kg}^{-1}$ reduziu consideravelmente a intensidade de percepção de dor (hipernocicepção) em camundongos swiss. 


\subsection{Estabilidade de ésteres de fósforo livres e coordenados a rutênio(II) em meio aquoso.}

\subsection{1 $\mathrm{P}(\mathrm{OR})_{3}$ e $\mathrm{P}(\mathrm{OH})(\mathrm{OR})_{2}$ em meio aquoso}

O tri-isopropil $\left(\mathrm{P}\left(\mathrm{OC}_{3} \mathrm{H}_{7}\right)_{3}\right)$, tributil $\left(\mathrm{P}\left(\mathrm{OC}_{4} \mathrm{H}_{9}\right)_{3}\right)$, trietil $\left(\mathrm{P}\left(\mathrm{OC}_{2} \mathrm{H}_{5}\right)_{3}\right)$, e trimetil $\left(\mathrm{P}\left(\mathrm{OCH}_{3}\right)_{3}\right)$ fosfitos foram monitorados separadamente por meio de ${ }^{1} \mathrm{H}$ RMN em função do tempo em meio aquoso pH 3,0 e $25 \pm 0,5{ }^{\circ} \mathrm{C}$. Entretanto, já no primeiro espectro destas

soluções apenas os deslocamentos químicos dos correspondentes dialquil fosfito $\left(\mathrm{P}(\mathrm{OH})(\mathrm{OR})_{2}\right)$ e álcool $(\mathrm{ROH})$ foram observados (reação 17). Isto inviabilizou seguir reação de hidrólise dos trialquil fosfitos nestas condições experimentais. Considerando que o primeiro espectro foi adquirido 20 minutos após a mistura do trialquil fosfito em solução aquosa, o limite superior de meia vida destes fosfitos pode ser estimado como $1,2 \times 10^{2} \operatorname{seg}\left(\mathrm{k} \sim 6 \times 10^{-3} \mathrm{~s}^{-1}\right)$.

$$
\mathrm{P}(\mathrm{OR})_{3}+\mathrm{H}_{2} \mathrm{O} \longrightarrow \mathrm{P}(\mathrm{OH})(\mathrm{OR})_{2}+\mathrm{ROH}
$$

A hidrólise do dietil fosfito $\left(\mathrm{P}(\mathrm{OH})\left(\mathrm{OC}_{2} \mathrm{H}_{5}\right)_{2}\right)$ também foi acompanhada em meio aquoso ( $\mathrm{pH} 1,0$ e 3,$0 ; 25 \pm 0,5^{\circ} \mathrm{C}$ ) por meio de ${ }^{1} \mathrm{H}$ RMN. Na Figura 19 são observados um quintupleto em 4,20 ppm e um tripleto em 1,35 ppm, os quais correspondem aos grupos $\mathrm{CH}_{2}$ e $\mathrm{CH}_{3}$ da molécula de dietil fosfito, respectivamente. O quintupleto é observado ao invés de um quadrupleto devido ao acoplamento ${ }^{3} \mathrm{~J}$ entre ${ }^{1} \mathrm{H} \mathrm{e}{ }^{31} \mathrm{P}$, que gera um duplo quarteto que, por sobreposição, resulta no quintupleto. Também é observado um dubleto em 5,17 e 8,77 ppm cuja constante de acoplamento ${ }^{1} \mathrm{~J}$, resultante do acoplamento entre os núcleos de ${ }^{1} \mathrm{H}$ e ${ }^{31} \mathrm{P}$, é igual a $720 \mathrm{~Hz}$. Este acoplamento ${ }^{1} \mathrm{~J}$ é característico da molécula de dietil fosfito na forma de fosfonato (reação 3 ) $[55 ; 102 ; 103]$. 
Figura 19 - Espectro de ${ }^{1} \mathrm{H}$ RMN para o dietil fosfito em $\mathrm{D}_{2} \mathrm{O}$ e $\mathrm{pH}=3,0\left(\mathrm{CD}_{3} \mathrm{COOD}\right)$ após 20 horas em solução, $\mathrm{C}_{\mathrm{P}(\mathrm{OH})(\mathrm{OEt})_{2}}=2,0 \times 10^{-4} \mathrm{~mol} \mathrm{~L}^{-1}$ e $25 \pm 0,5{ }^{\circ} \mathrm{C}$; (a) dietil fosfito, (b) monoetil fosfito e (c) etanol

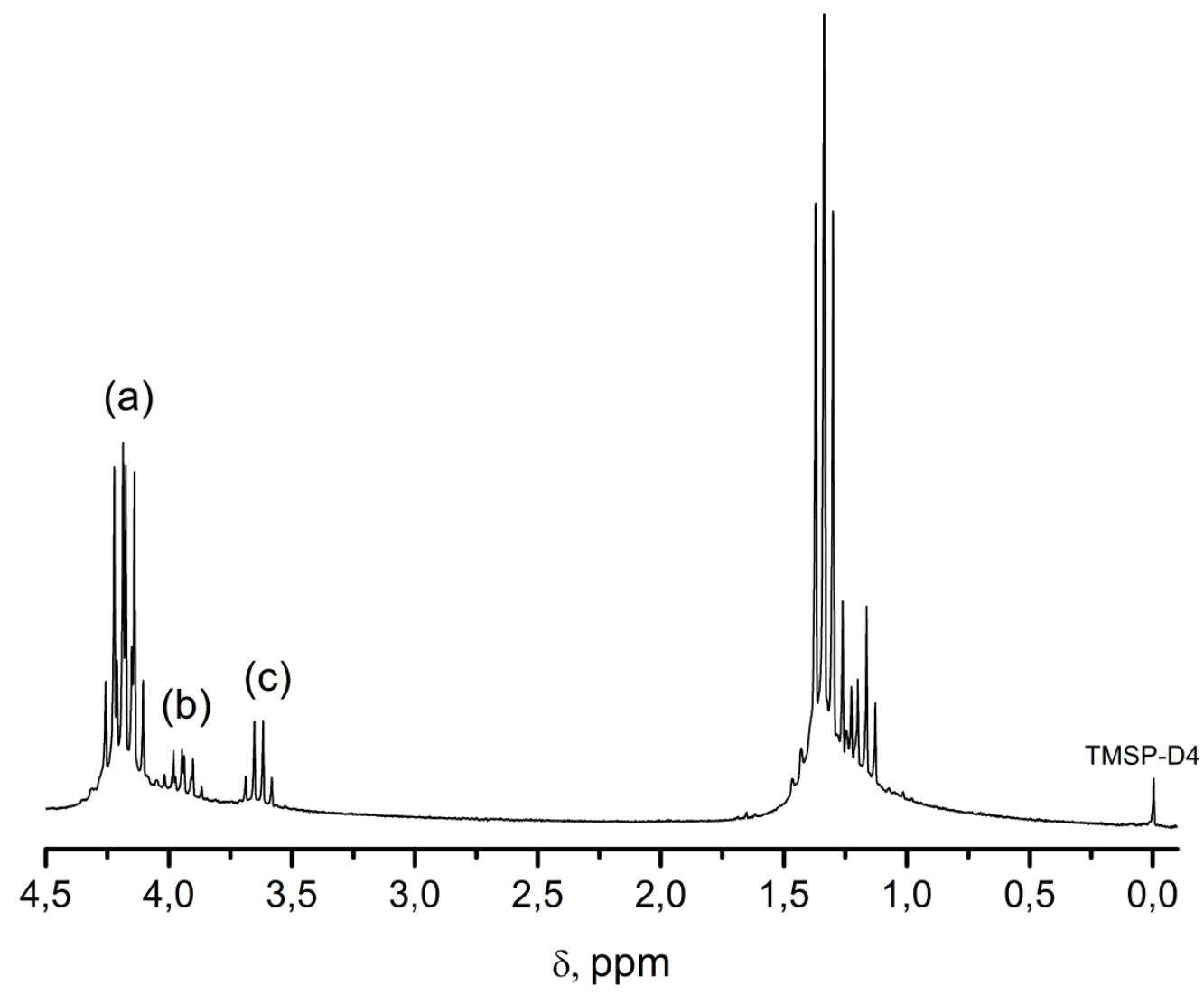

Em ambas as concentrações hidrogeniônicas (pH 1,0 e 3,0), além dos sinais referentes a molécula de dietil fosfito, o espectro apresentou em função do tempo um quadrupleto em 3,64 ppm e um tripleto em 1,17 ppm referentes aos grupos $\mathrm{CH}_{2}$ e $\mathrm{CH}_{3}$ da molécula de etanol (Figura 19 c). Esta atribuição foi confirmada por adição de etanol na amostra. Os picos de etanol tiveram suas intensidades aumentadas em função do tempo, enquanto que os picos correspondentes à molécula de dietil fosfito tiveram suas intensidades diminuídas, o que indica a hidrólise do dietil fosfito neste meio. Um quintupleto em 3,94 ppm e um tripleto em 1,26 ppm também foram observados devido a conversão do dietil fosfito em monoetil fosfito $\left(\mathrm{P}(\mathrm{OH})_{2}\left(\mathrm{OCH}_{2} \mathrm{CH}_{3}\right)\right)$, Figura 19 b, o qual, em seguida se converte em ácido fosforoso $\left(\mathrm{P}(\mathrm{OH})_{3}\right)[70]$ 
A partir dos dados cinéticos de RMN (decaimento da área do quintupleto em 4,20 ppm), as constantes de velocidade (k) para o decaimento da molécula de dietil fosfito (reação 18) em $\mathrm{pH} \quad 1,0$ e $3,0 \quad\left(25 \quad \pm \quad 0,5^{\circ} \mathrm{C}\right)$ foram calculadas como $1,8 \times 10^{-4} \mathrm{~s}^{-1}$ $\left(\mathrm{t}_{1 / 2} \sim 1,1 \mathrm{~h}\right)$ e $7,1 \times 10^{-6} \mathrm{~s}^{-1}\left(\mathrm{t}_{1 / 2} \sim 27,0 \mathrm{~h}\right)$, respectivamente. A velocidade de decaimento da molécula de dietil fosfito é mais alta em $\mathrm{pH} 1,0$ do que em $\mathrm{pH} 3,0$, o que está em concordância com a proposta da hidrólise ser promovida por catálise ácida (Figura 2c/d) [61; 104].

$$
\mathrm{P}(\mathrm{OH})\left(\mathrm{OCH}_{2} \mathrm{CH}_{3}\right)_{2}+\mathrm{H}_{2} \mathrm{O} \longrightarrow \mathrm{P}(\mathrm{OH})_{2}\left(\mathrm{OCH}_{2} \mathrm{CH}_{3}\right)+\mathrm{CH}_{3} \mathrm{CH}_{2} \mathrm{OH}
$$

De acordo com cálculos teóricos realizados por Guthrie [55], a energia livre $\left(\Delta \mathrm{G}^{0}\right)$ para a hidrólise de ésteres de fósforo(III) aumenta da seguinte forma: $\mathrm{P}\left(\mathrm{OCH}_{2} \mathrm{CH}_{3}\right)_{3}<$ $\mathrm{P}(\mathrm{OH})\left(\mathrm{OCH}_{2} \mathrm{CH}_{3}\right)_{2}<\mathrm{P}(\mathrm{OH})_{2}\left(\mathrm{OCH}_{2} \mathrm{CH}_{3}\right)$ [55]. De fato, comparando-se a hidrólise do trietil fosfito com a do dietil fosfito, observa-se uma diferença considerável na escala de tempo de reação (de segundos para horas).

Neste estudo dos fosfitos livres, a ressonância magnética nuclear de ${ }^{1} \mathrm{H}$ foi utilizada ao invés da ${ }^{31} \mathrm{P}$ RMN, uma vez que o espectro é prontamente adquirido, os deslocamentos químicos são facilmente identificados e suficientemente separados, o que permite o cálculo da área de cada sinal de forma eficiente.

\subsection{2 $\mathrm{P}(\mathrm{OR})_{3}$ e $\mathrm{P}(\mathrm{OH})(\mathrm{OR})_{2}$ coordenados aos fragmentos trans $-\left[\mathrm{Ru}\left(\mathrm{H}_{2} \mathrm{O}\right)\left(\mathrm{NH}_{3}\right)_{4}\right]^{2+}$ e trans- $\left[\mathrm{Ru}\left(\mathrm{NO}^{+}\right)\left(\mathrm{NH}_{3}\right)_{4}\right]^{3+}$ em meio aquoso}

Dados espectrofotométricos indicam que o íon trans- $\left[\mathrm{Ru}\left(\mathrm{H}_{2} \mathrm{O}\right)\left(\mathrm{NH}_{3}\right)_{4}\left(\mathrm{P}\left(\mathrm{OC}_{2} \mathrm{H}_{5}\right)_{3}\right)\right]^{2+}$ é estável por 2 semanas $\left(\mathrm{k}>5 \times 10^{-7} \mathrm{em}\right.$ pH 3,0 e $\left.25 \pm 0,1^{\circ} \mathrm{C}\right)$ [67]. No íon 
trans- $\left[\mathrm{Ru}\left(\mathrm{NH}_{3}\right)_{4}\left(\mathrm{P}\left(\mathrm{OC}_{2} \mathrm{H}_{5}\right)_{3}\right)_{2}\right]^{2+}$ a hidrólise do trietil fosfito coordenado não é observada,

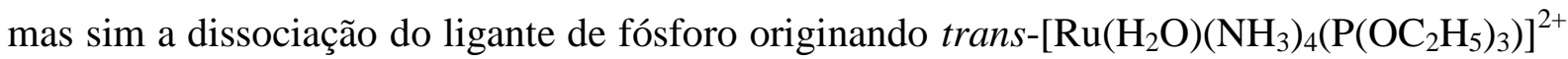
$\left(\mathrm{k}=2,6 \times 10^{-5} \mathrm{~s}^{-1}\right.$ em pH 3,0 e $\left.25 \pm 0,1^{\circ} \mathrm{C}[67]\right)$.

Neste trabalho os íons complexos trans- $\left[\mathrm{Ru}\left(\mathrm{H}_{2} \mathrm{O}\right)\left(\mathrm{NH}_{3}\right)_{4}\left(\mathrm{P}\left(\mathrm{OC}_{3} \mathrm{H}_{7}\right)_{3}\right)\right]^{2+}$, trans- $\left[\mathrm{Ru}\left(\mathrm{H}_{2} \mathrm{O}\right)\left(\mathrm{NH}_{3}\right)_{4}\left(\mathrm{P}\left(\mathrm{OC}_{4} \mathrm{H}_{9}\right)_{3}\right)\right]^{2+}$ e trans- $\left[\mathrm{Ru}\left(\mathrm{H}_{2} \mathrm{O}\right)\left(\mathrm{NH}_{3}\right)_{4}\left(\mathrm{P}(\mathrm{OH})\left(\mathrm{OCH}_{2} \mathrm{CH}_{3}\right)_{2}\right)\right]^{2+}$ foram investigados com respeito à hidrólise sob as mesmas condições experimentais que as moléculas de fosfito livres ( $\mathrm{pH} 3,0$ e $\left.25,0 \pm 0,5^{\circ} \mathrm{C}\right)$.

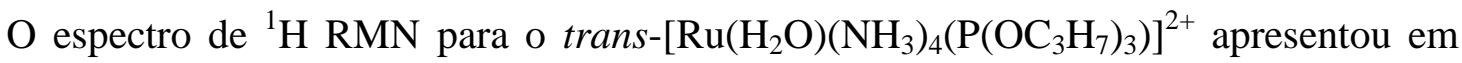
função do tempo um decréscimo da área do dubleto em 1,30 ppm, correspondente aos grupos $\mathrm{CH}_{3}$ do tri-isopropil fosfito coordenado, e um aumento da área do dubleto em 1,16 ppm dos grupos $\mathrm{CH}_{3}$ do isopropanol. Assim, a constante de hidrólise do tri-isopropil coordenado ao aqua complexo (reação 19) foi calculada como $\mathrm{k}=4,45 \times 10^{-7} \mathrm{~s}^{-1}$ $\left(\mathrm{t}_{1 / 2} \sim 18\right.$ dias $)$.

trans- $\left[\mathrm{Ru}\left(\mathrm{H}_{2} \mathrm{O}\right)\left(\mathrm{NH}_{3}\right)_{4} \mathrm{P}(\mathrm{OR})_{3}\right]^{2+}+\mathrm{H}_{2} \mathrm{O} \longrightarrow$ trans $-\left[\mathrm{Ru}\left(\mathrm{H}_{2} \mathrm{O}\right)\left(\mathrm{NH}_{3}\right)_{4} \mathrm{P}(\mathrm{OH})(\mathrm{OR})_{2}\right]^{2+}+\mathrm{ROH}$

De forma similar, para o trans- $\left[\mathrm{Ru}\left(\mathrm{H}_{2} \mathrm{O}\right)\left(\mathrm{NH}_{3}\right)_{4}\left(\mathrm{P}\left(\mathrm{OC}_{4} \mathrm{H}_{9}\right)_{3}\right)\right]^{2+}$ a área do quadrupleto (ao invés de um tripleto devido ao acoplamento ${ }^{3} \mathrm{~J}$ entre ${ }^{1} \mathrm{H}$ e ${ }^{31} \mathrm{P}$ ) em 4,03 ppm, correspondente ao grupo $\alpha-\mathrm{CH}_{2}$ do tributil fosfito coordenado, decresce em função do tempo, enquanto a área do tripleto $(3,49 \mathrm{ppm})$ do grupo $\alpha-\mathrm{CH}_{2}$ do butanol aumenta. A constante de

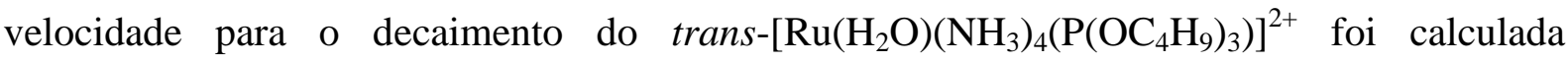
como $\mathrm{k}=7,92 \times 10^{-7} \mathrm{~s}^{-1}\left(\mathrm{t}_{1 / 2} \sim 10\right.$ dias $)$.

A análise do espectro de ${ }^{1} \mathrm{H}$ RMN do trans- $\left[\mathrm{Ru}\left(\mathrm{H}_{2} \mathrm{O}\right)\left(\mathrm{NH}_{3}\right)_{4}\left(\mathrm{P}(\mathrm{OH})\left(\mathrm{OCH}_{2} \mathrm{CH}_{3}\right)_{2}\right)\right]^{2+}$ também permite observar que, em função do tempo, os picos do dietil fosfito (4,20 e 1,35 ppm) diminuem e, concomitantemente, ocorre a formação dos picos do monoetil 
fosfito (3,94 e 1,26 ppm) e etanol (3,64 e 1,17 ppm). Apesar dos deslocamentos químicos para estas espécies serem similares aos observados para a molécula de dietil fosfito livre, neste caso nenhum acoplamento ${ }^{1} \mathbf{J}$ entre ${ }^{1} \mathrm{H}$ e ${ }^{31} \mathrm{P}$ foi observado. Isto é consistente com a coordenação do dietil fosfito em sua forma piramidal (fósforo trivalente) [55; 70]. De acordo com os dados de ${ }^{1} \mathrm{H}$ RMN, a hidrólise do dietil fosfito coordenado ao complexo trans- $\left[\mathrm{Ru}\left(\mathrm{H}_{2} \mathrm{O}\right)\left(\mathrm{NH}_{3}\right)_{4}\left(\mathrm{P}(\mathrm{OH})\left(\mathrm{OCH}_{2} \mathrm{CH}_{3}\right)_{2}\right)\right]^{2+} \quad($ reação 20$)$ ocorre com constante de velocidade de $4,8 \times 10^{-7} \mathrm{~s}^{-1}\left(\mathrm{t}_{1 / 2} \sim 17\right.$ dias $)$ em $\mathrm{pH} 3,0$ e $25 \pm 0,5^{\circ} \mathrm{C}$.

$$
\begin{aligned}
\text { trans }-\left[\mathrm{Ru}\left(\mathrm{H}_{2} \mathrm{O}\right)\left(\mathrm{NH}_{3}\right)_{4} \mathrm{P}(\mathrm{OH})\left(\mathrm{OCH}_{2} \mathrm{CH}_{3}\right)_{2}\right]^{2+}+\mathrm{H}_{2} \mathrm{O} \\
\longrightarrow \text { trans }-\left[\mathrm{Ru}\left(\mathrm{H}_{2} \mathrm{O}\right)\left(\mathrm{NH}_{3}\right)_{4} \mathrm{P}(\mathrm{OH})_{2}\left(\mathrm{OCH}_{2} \mathrm{CH}_{3}\right)\right]^{2+}+\mathrm{CH}_{3} \mathrm{CH}_{2} \mathrm{OH}
\end{aligned}
$$

O espectro de ${ }^{31} \mathrm{P}$ RMN do trans- $\left[\mathrm{Ru}\left(\mathrm{H}_{2} \mathrm{O}\right)\left(\mathrm{NH}_{3}\right)_{4} \mathrm{P}(\mathrm{OH})\left(\mathrm{OCH}_{2} \mathrm{CH}_{3}\right)_{2}\right]^{2+}$ em pH 3,0 apresentou dois picos de fósforo(III) coordenado em 150,1 e 142,0 ppm. Estes picos podem ser explicados com base na presença de um próton ácido (grupo hidroxila) no ligante de fósforo. Assim, o pico em 150,1 ppm corresponderia ao trans- $\left[\mathrm{Ru}\left(\mathrm{H}_{2} \mathrm{O}\right)\left(\mathrm{NH}_{3}\right)_{4} \mathrm{P}(\mathrm{OH})\left(\mathrm{OCH}_{2} \mathrm{CH}_{3}\right)_{2}\right]^{2+} \quad$ e $\quad$ o $\quad$ pico $\quad$ em $142,0 \quad$ ppm ao trans-[Ru( $\left.\left.\mathrm{H}_{2} \mathrm{O}\right)\left(\mathrm{NH}_{3}\right)_{4} \mathrm{P}(\mathrm{O})\left(\mathrm{OCH}_{2} \mathrm{CH}_{3}\right)_{2}\right]^{+}$. $\mathrm{O}$ cálculo da área destes picos permite estimar o pK $_{\text {a }}$ para a reação 21 como igual a 3,6. Este valor está em concordância com o valor de 3,7 calculado previamente por meio de voltametria cíclica [56].

$$
\begin{gathered}
\text { trans- }\left[\mathrm{Ru}\left(\mathrm{H}_{2} \mathrm{O}\right)\left(\mathrm{NH}_{3}\right)_{4} \mathrm{P}(\mathrm{OH})\left(\mathrm{OCH}_{2} \mathrm{CH}_{3}\right)_{2}\right]^{2+}+\mathrm{H}_{2} \mathrm{O} \\
\rightleftharpoons \text { trans }-\left[\mathrm{Ru}\left(\mathrm{H}_{2} \mathrm{O}\right)\left(\mathrm{NH}_{3}\right)_{4} \mathrm{P}(\mathrm{O})\left(\mathrm{OCH}_{2} \mathrm{CH}_{3}\right)_{2}\right]^{+}+\mathrm{H}_{3} \mathrm{O}^{+}
\end{gathered}
$$

A estabilidade do tri-isopropil $\left(\mathrm{P}\left(\mathrm{OC}_{3} \mathrm{H}_{7}\right)_{3}\right)$, tributil $\left(\mathrm{P}\left(\mathrm{OC}_{4} \mathrm{H}_{9}\right)_{3}\right)$, trietil $\left(\mathrm{P}\left(\mathrm{OC}_{2} \mathrm{H}_{5}\right)_{3}\right)$, e trimetil $\left(\mathrm{P}\left(\mathrm{OCH}_{3}\right)_{3}\right)$ fosfitos coordenados ao fragmento trans- $\left[\mathrm{Ru}(\mathrm{NO})\left(\mathrm{NH}_{3}\right)_{4}\right]^{3+}$ também foi 
avaliada em meio aquoso. Ao contrário do observado para as moléculas livres, os deslocamentos químicos de ${ }^{1} \mathrm{H}$ RMN para estes nitrosilos complexos e seus produtos de hidrólise são próximos, o que dificulta a identificação e o cálculo da área. Dessa forma, a ${ }^{31} \mathrm{P}$ RMN foi utilizada para acompanhar as reações nestes complexos.

$\mathrm{O}$ íon trans- $\left[\mathrm{Ru}(\mathrm{NO})\left(\mathrm{NH}_{3}\right)_{4} \mathrm{P}\left(\mathrm{OCH}_{3}\right)_{3}\right]^{3+}$, em solução $\mathrm{pH} 3,0\left(25 \pm 0,5^{\circ} \mathrm{C}\right)$, apresentou em seu espectro de ${ }^{31} \mathrm{P}$ RMN apenas um pico em $85 \mathrm{ppm}$, o qual decai com $\mathrm{k}=3,01 \times 10^{-5} \mathrm{~s}^{-1}$ $\left(\mathrm{t}_{1 / 2}=6,4 \mathrm{~h}\right)$. Concomitantemente, um pico em 71 ppm relativo à espécie trans- $\left[\mathrm{Ru}(\mathrm{NO})\left(\mathrm{NH}_{3}\right)_{4} \mathrm{P}(\mathrm{OH})\left(\mathrm{OCH}_{3}\right)_{2}\right]^{3+}$ é formado com a mesma constante de velocidade $\left(\mathrm{k}=3,03 \times 10^{-5} \mathrm{~s}^{-1}\right)$, Figura 20.

Figura 20 - trans- $\left[\mathrm{Ru}(\mathrm{NO})\left(\mathrm{NH}_{3}\right)_{4}\left(\mathrm{P}\left(\mathrm{OCH}_{3}\right)_{3}\right)\right]^{3+}$ em $\mathrm{D}_{2} \mathrm{O}, \mathrm{pH}=3,0\left(\mathrm{CD}_{3} \mathrm{COOD}\right)$ e $25 \pm 0,5$ ${ }^{\circ} \mathrm{C}$ (a) Espectro de ${ }^{31} \mathrm{P} \mathrm{RMN}\left(\mathrm{NH}_{4} \mathrm{PF}_{6}\right.$ como referência interna); (b).Variação da área dos picos de ${ }^{31} \mathrm{P}$ RMN em função do tempo.

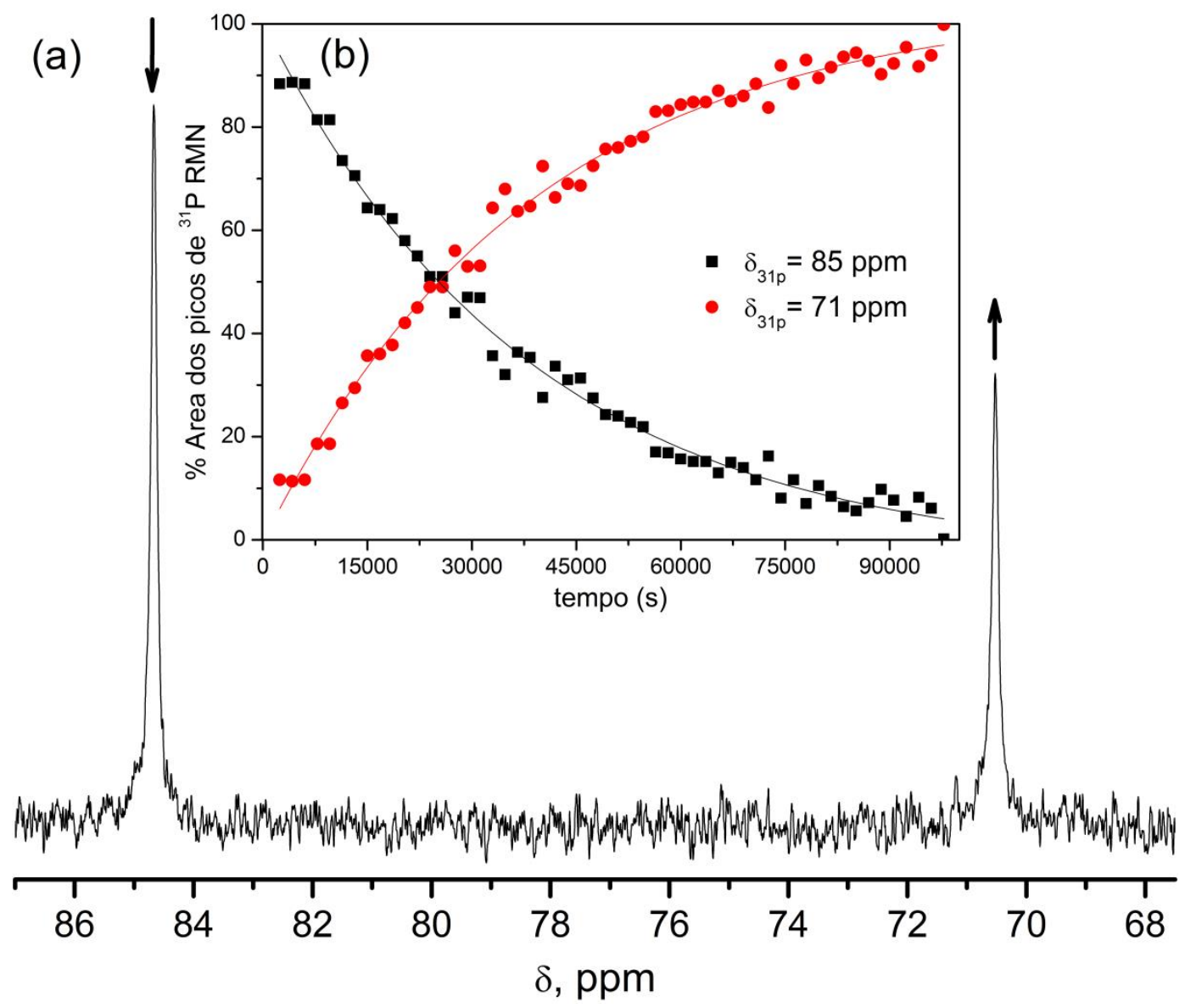


$\mathrm{O}$ decaimento da espécie trans- $\left[\mathrm{Ru}(\mathrm{NO})\left(\mathrm{NH}_{3}\right)_{4}\left(\mathrm{P}\left(\mathrm{OCH}_{3}\right)_{3}\right)\right]^{3+}$ também foi monitorado por meio de espectroscopia de infravermelho em solução aquosa pH 3,0. Na Figura 21 é possível observar que a frequência de estiramento $\mathrm{NO}^{+}$em $1922 \mathrm{~cm}^{-1}$ diminui em função do tempo originando concomitantemente uma nova $v_{\mathrm{NO}^{+}}$em $1891 \mathrm{~cm}^{-1}$. O ponto isosbéstico na Figura 21 sugere que o $\mathrm{P}\left(\mathrm{OCH}_{3}\right)_{3}$ coordenado ao fragmento $\left[\mathrm{Ru}(\mathrm{NO})\left(\mathrm{NH}_{3}\right)_{4}\right]^{3+}$ sofre hidrólise gerando quantitativamente trans- $\left[\mathrm{Ru}(\mathrm{NO})\left(\mathrm{NH}_{3}\right)_{4}\left(\mathrm{P}(\mathrm{OH})\left(\mathrm{OCH}_{3}\right)_{2}\right)\right]^{3+}$ (reação 22).

trans $-\left[\mathrm{Ru}(\mathrm{NO})\left(\mathrm{NH}_{3}\right)_{4} \mathrm{P}(\mathrm{OR})_{3}\right]^{3+}+\mathrm{H}_{2} \mathrm{O} \longrightarrow$ trans $-\left[\mathrm{Ru}(\mathrm{NO})\left(\mathrm{NH}_{3}\right)_{4} \mathrm{P}(\mathrm{OH})(\mathrm{OR})_{2}\right]^{3+}+\mathrm{ROH}$

Figura 21 - Espectro de infravermelho para o trans- $\left[\mathrm{Ru}(\mathrm{NO})\left(\mathrm{NH}_{3}\right)_{4}\left(\mathrm{P}\left(\mathrm{OCH}_{3}\right)_{3}\right)\right]^{3+}$ em pH 3,0, $\mu=0,1 \mathrm{~mol} \mathrm{~L}^{-1}, 25 \pm 1,0{ }^{\circ} \mathrm{C}$.

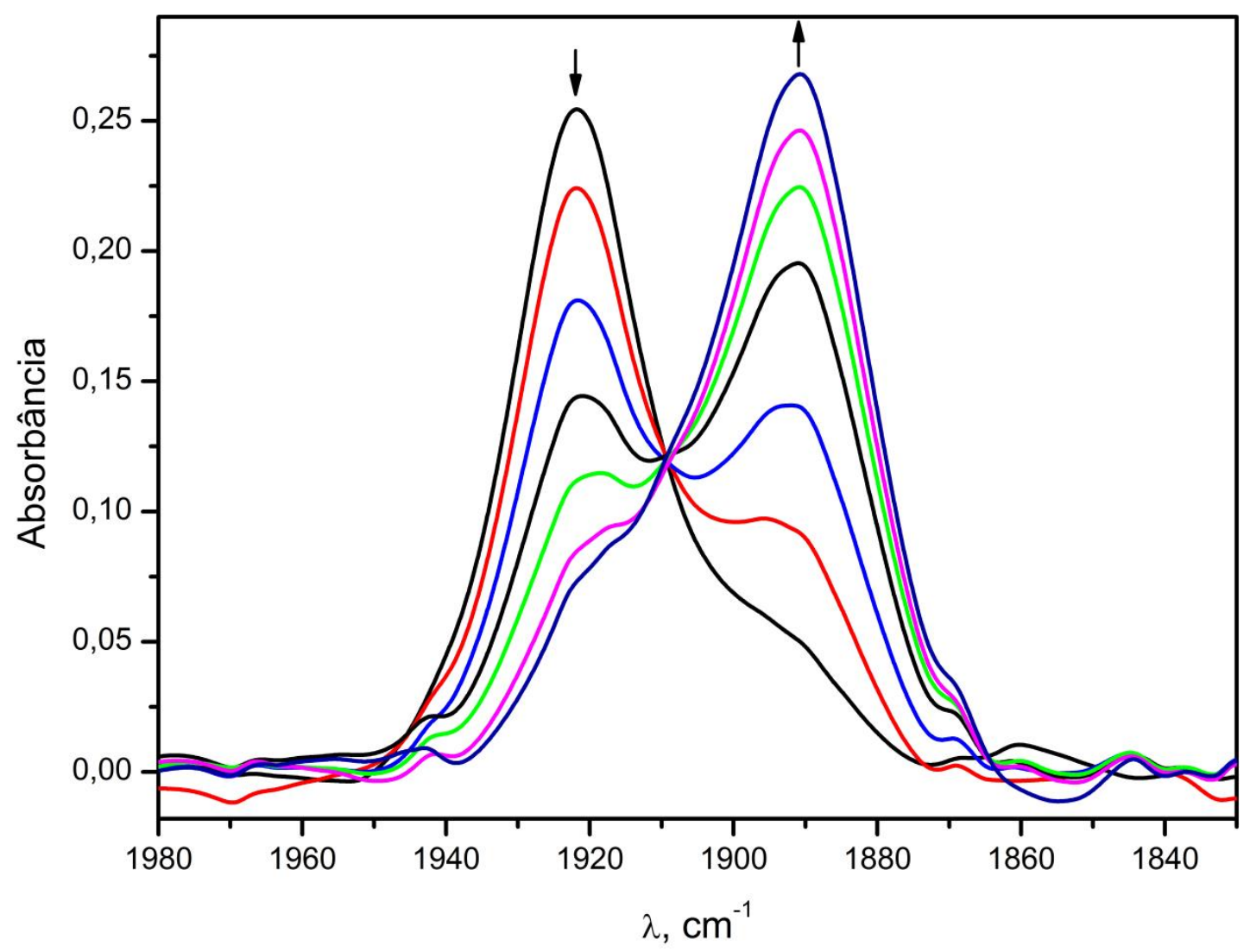


Este mesmo comportamento é observado para os nitrosilos complexos de trietil, triisopropil e tributil fosfito (reação 22). As constantes de velocidade de hidrólise para cada complexo em pH 3,0 e 25,0 $\pm 0,5^{\circ} \mathrm{C}$ são apresentadas na Tabela 7.

Todos os trialquil fosfitos e o dietil fosfito são substancialmente estabilizados com respeito à hidrólise quando coordenados ao centro metálico de $\mathrm{Ru}(\mathrm{II})$. $\mathrm{O}$ tri-isopropil, tributil e dietil fosfitos coordenados ao fragmento trans- $\left[\mathrm{Ru}\left(\mathrm{H}_{2} \mathrm{O}\right)\left(\mathrm{NH}_{3}\right)_{4}\right]^{2+}$ apresentaram constante de hidrólise da mesma ordem de magnitude $\left(4,4-7,9 \times 10^{-7} \mathrm{~s}^{-1}\right)$. Este aumento na estabilidade frente à hidrólise nas moléculas de fosfito coordenadas ao aqua complexo de rutênio pode ser explicada com base na retrodoação $\mathrm{Ru}(\mathrm{II}) \rightarrow \mathrm{P}(\mathrm{III})$, a qual aumenta a densidade eletrônica sobre o ligante de fósforo e, portanto, o desativa quanto ao ataque nucleofílico e consequente hidrólise.

Tabela 7 - Constante de velocidade de hidrólise para trialquil fosfitos coordenados ao fragmento trans- $\left[\mathrm{Ru}(\mathrm{NO})\left(\mathrm{NH}_{3}\right)_{4}\right]^{3+}$ em $\mathrm{pH} 3,0$ e $25,0 \pm 0,5^{\circ} \mathrm{C}$

\begin{tabular}{lc}
\hline \multicolumn{1}{c}{ Complexo } & $\mathbf{k}\left(\mathrm{s}^{-1}\right)$ \\
\hline trans $-\left[\mathrm{Ru}(\mathrm{NO})\left(\mathrm{NH}_{3}\right)_{4}\left(\mathrm{P}\left(\mathrm{OCH}_{3}\right)_{3}\right)\right]^{3+}$ & $3,01 \times 10^{-5}$ \\
trans $-\left[\mathrm{Ru}(\mathrm{NO})\left(\mathrm{NH}_{3}\right)_{4}\left(\mathrm{P}\left(\mathrm{OC}_{2} \mathrm{H}_{5}\right)_{3}\right)\right]^{3+}$ & $2,90 \times 10^{-6}$ \\
trans $-\left[\mathrm{Ru}(\mathrm{NO})\left(\mathrm{NH}_{3}\right)_{4}\left(\mathrm{P}\left(\mathrm{OC}_{3} \mathrm{H}_{7}\right)_{3}\right)\right]^{3+}$ & $1,35 \times 10^{-5}$ \\
trans $-\left[\mathrm{Ru}(\mathrm{NO})\left(\mathrm{NH}_{3}\right)_{4}\left(\mathrm{P}\left(\mathrm{OC}_{4} \mathrm{H}_{9}\right)_{3}\right)\right]^{3+}$ & $6,04 \times 10^{-6}$ \\
trans $-\left[\mathrm{Ru}(\mathrm{NO})\left(\mathrm{NH}_{3}\right)_{4}\left(\mathrm{P}(\mathrm{O})(\mathrm{OH})_{2}\right)\right]^{2+}$ & $2,10 \times 10^{-4 *}[59]$ \\
trans $-\left[\mathrm{Ru}(\mathrm{NO})\left(\mathrm{NH}_{3}\right)_{4}\left(\mathrm{P}(\mathrm{O})\left(\mathrm{OC}_{2} \mathrm{H}_{5}\right)_{2}\right)\right]^{2+}$ & $8,90 \times 10^{-7} * *$
\end{tabular}

Constante de velocidade calculada para o decaimento do nitrosilo complexo em consequência da isomerização* e da dissociação** do ligante de fósforo.

A extensão da retrodoação $\mathrm{Ru}(\mathrm{II}) \rightarrow \mathrm{P}(\mathrm{III})$ nos compostos trans-[Ru(L) $\left.\left(\mathrm{NH}_{3}\right)_{4} \mathrm{P}(\mathrm{III})\right]^{\mathrm{n}+}$ é dependente do ligante (L) em posição trans ao éster de fósforo. Em nitrosilos complexos esta retrodoação tende a ser menor do que nos aquo complexos devido ao forte caráter $\pi$-aceptor do ligante nitrosônio que ocasiona uma competição pelos elétrons $4 \mathrm{~d} \pi$ do $\mathrm{Ru}(\mathrm{II})$ diminuindo a densidade eletrônica sobre o fosfito. Esta diferença na extensão de retrodoação 
$\mathrm{Ru}(\mathrm{II}) \rightarrow \mathrm{P}(\mathrm{III})$ suporta $\mathrm{o}$ fato da hidrólise ocorrer de forma mais rápido nos íons trans- $\left[\mathrm{Ru}(\mathrm{NO})\left(\mathrm{NH}_{3}\right)_{4} \mathrm{P}(\mathrm{III})\right]^{3+}$ do que nos trans-[Ru($\left.\left(\mathrm{H}_{2} \mathrm{O}\right)\left(\mathrm{NH}_{3}\right)_{4} \mathrm{P}(\mathrm{III})\right]^{2+}$. Nota-se também que para os nitrosilos complexos uma faixa de constante de hidrólise mais ampla $\left(3,00-0,29 \times 10^{-6} \mathrm{~s}^{-1}\right)$ foi observada, indicando uma capacidade de modulação maior do que nos aquo complexos.

Embora os ligantes trialquil fosfitos nos íons trans- $\left[\mathrm{Ru}\left(\mathrm{H}_{2} \mathrm{O}\right)\left(\mathrm{NH}_{3}\right)_{4} \mathrm{P}(\mathrm{OR})_{3}\right]^{2+} \mathrm{e}$ trans-[Ru(NO) $\left.\left(\mathrm{NH}_{3}\right)_{4} \mathrm{P}(\mathrm{OR})_{3}\right]^{3+}$ hidrolisem dando origem aos respectivos dialquil fosfito complexos, no íon trans- $\left[\mathrm{Ru}\left(\mathrm{NH}_{3}\right)_{4}\left(\mathrm{P}(\mathrm{OR})_{3}\right)_{2}\right]^{2+}$ estes mesmos trialquil fosfitos não hidrolisam enquanto coordenados. Ao invés da hidrólise, a dissociação de um dos grupos trialquil fosfitos é observada $\left(\mathrm{k}=1,90-8,02 \times 10^{-5} \mathrm{~s}^{-1}\right.$ em $\mathrm{pH} 3,0$ e $25 \pm 0,5^{\circ} \mathrm{C}$ para trans- $\left[\mathrm{Ru}\left(\mathrm{NH}_{3}\right)_{4}\left(\mathrm{P}(\mathrm{OR})_{3}\right)_{2}\right]^{2+}$, em que $\mathrm{P}(\mathrm{OR})=\mathrm{P}\left(\mathrm{OC}_{3} \mathrm{H}_{7}\right)_{3}, \mathrm{P}\left(\mathrm{OC}_{4} \mathrm{H}_{9}\right)_{3}, \mathrm{P}\left(\mathrm{OC}_{2} \mathrm{H}_{5}\right)_{3}$ e $\mathrm{P}\left(\mathrm{OCH}_{3}\right)_{3}$ [66; 67]). Da mesma maneira, apesar da hidrólise ser observada no íon trans- $\left[\mathrm{Ru}\left(\mathrm{H}_{2} \mathrm{O}\right)\left(\mathrm{NH}_{3}\right)_{4}\left(\mathrm{P}(\mathrm{OH})\left(\mathrm{OCH}_{2} \mathrm{CH}_{3}\right)_{2}\right)\right]^{2+}$, no trans- $\left[\mathrm{Ru}(\mathrm{NO})\left(\mathrm{NH}_{3}\right)_{4}\left(\mathrm{P}(\mathrm{O})\left(\mathrm{OCH}_{2} \mathrm{CH}_{3}\right)_{2}\right)\right]^{2+} \mathrm{o}$ ligante dietil fosfito desprotonado também se dissocia sem prévia hidrólise, conforme discutido na seção 4.2 (Tabela 7).

Ambas as reações, hidrólise e dissociação, podem ocorrer nos ligantes ésteres de fósforo. Quando $k_{\text {diss }}>k_{\text {hid }}$ a reação de dissociação será observada e, quando $k_{\text {diss }}<k_{\text {hid }}$ a reação de hidrólise será observada. A reação mais favorável (hidrólise ou dissociação) em cada complexo provavelmente está relacionada a fatores intrínsecos do sistema como o grau de suscetibilidade ao ataque nucleofílico do ligante de fósforo e o efeito trans exercido pelo ligante trans posicionado ao fósforo.

Dessa forma, sabendo-se que a constante de dissociação do dietil fosfito no complexo trans- $\left[\mathrm{Ru}(\mathrm{NO})\left(\mathrm{NH}_{3}\right)_{4}\left(\mathrm{P}(\mathrm{O})\left(\mathrm{OCH}_{2} \mathrm{CH}_{3}\right)_{2}\right)\right]^{2+}$ é $\mathrm{k}_{\text {diss }}=8,90 \times 10^{-7} \mathrm{~s}^{-1}$, então pode-se admitir que a constante de velocidade de hidrólise para este complexo será $\mathrm{k}_{\text {hid }}<8,90 \times 10^{-7} \mathrm{~s}^{-1}$. Assim, a estabilidade com respeito a hidrólise nos nitrosilos complexo de ésteres de fósforo segue a 
sequência: $\mathrm{P}(\mathrm{O})\left(\mathrm{OC}_{2} \mathrm{H}_{5}\right)_{2}>\mathrm{P}\left(\mathrm{OC}_{2} \mathrm{H}_{5}\right)_{3}>\mathrm{P}\left(\mathrm{OC}_{4} \mathrm{H}_{9}\right)_{3}>\mathrm{P}\left(\mathrm{O}^{\mathrm{i}} \mathrm{C}_{3} \mathrm{H}_{7}\right)_{3}>\mathrm{P}\left(\mathrm{OCH}_{3}\right)_{3}$. Esta sequência de estabilidade não coincide com a sequência de deslocamentos químicos de ${ }^{31} \mathrm{P}$ NMR observada para estes complexos: 68, 80, 79, 75 e 85 ppm. No entanto, exceto pelo $\mathrm{P}\left(\mathrm{OCH}_{3}\right)_{3}\left(\delta_{13 \mathrm{C}}=61 \mathrm{ppm}\right)$, a sequencia de estabilidade se correlaciona com deslocamentos químicos dos átomos de carbonos $\alpha: \delta_{13 \mathrm{C}}=64,70,75,81 \mathrm{ppm}$ (Figura 22).

Figura 22 - Correlação entre os valores de $\mathrm{k}_{\mathrm{hid}}$ e $\delta_{13 \mathrm{C}}(\mathrm{ppm})$ em nitrosilos complexos de rutênio

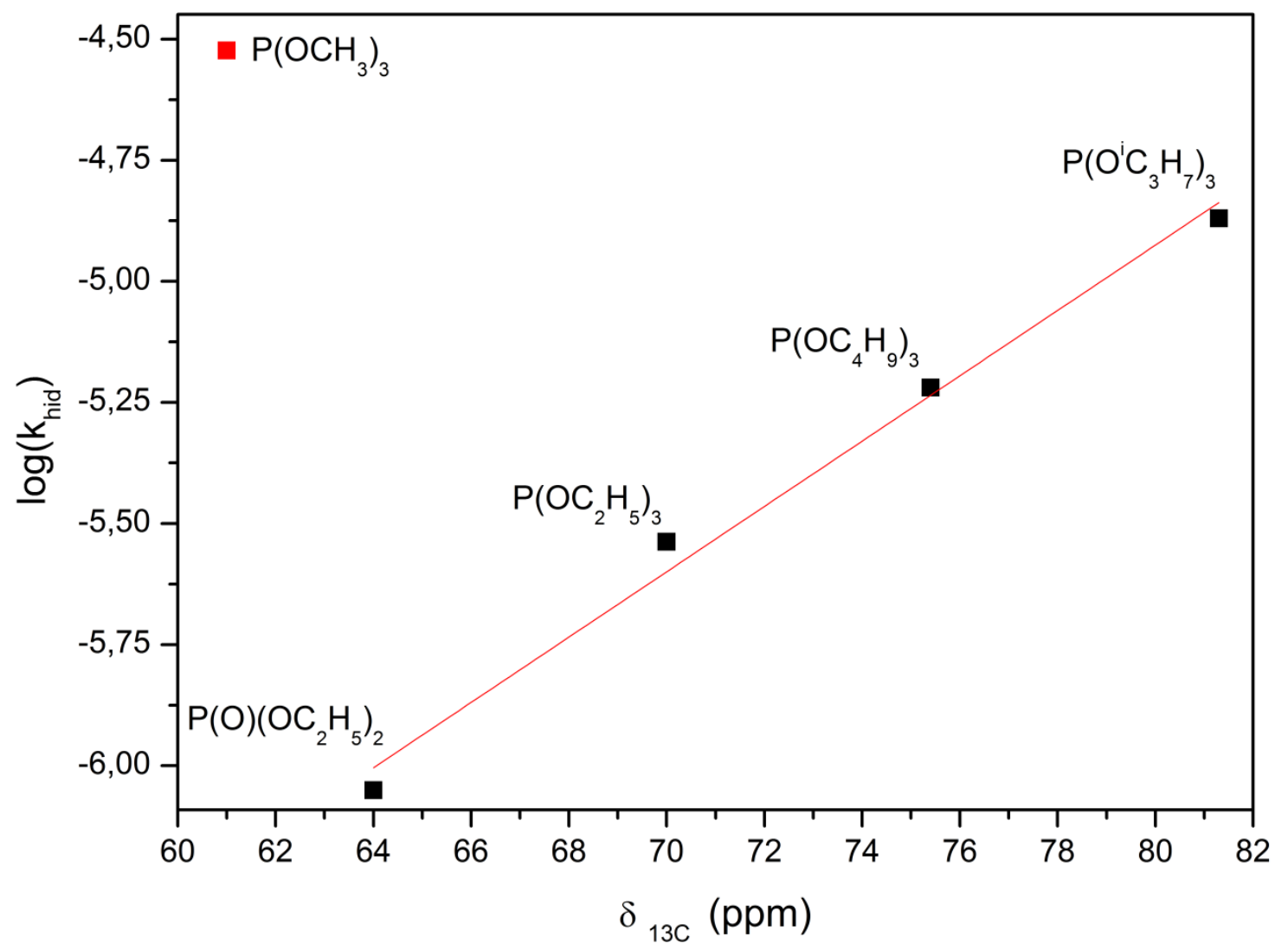

A correlação entre a constante de hidrólise e o $\delta_{13 \mathrm{C}}$ indica que nos íons trans-[Ru(NO)(NH$\left.)_{4} \mathrm{P}(\mathrm{III})\right]^{3+}$, nos quais o éster de fosforo já encontra-se ativado pela ligação Ru-P, o ataque nucleofílico promovido pela molécula de água é mais favorável no carbono $\alpha$ do que no átomo de fósforo do fosfito (Figura 23 a). Diferentemente, no íon 
trans- $\left[\mathrm{Ru}(\mathrm{NO})\left(\mathrm{NH}_{3}\right)_{4} \mathrm{P}\left(\mathrm{OCH}_{3}\right)_{3}\right]^{3+}$, que apresenta o átomo de fósforo mais positivo $\left(\delta_{31 \mathrm{P}}=84 \mathrm{ppm}\right)$ e o menor efeito estérico entre os composto estudados, provavelmente o ataque nucleofílico ocorra no átomo de fósforo conforme a Figura $23 \mathrm{~b}$.

Figura 23 - Mecanismo de hidrólise de fosfitos em nitrosilos complexos de rutênio (a) Quebra da ligação O-C (Mecanismo de Michaelis-Arbusov), (b) Quebra da ligação P-O (Mecanismo de Aksnes)

(a)

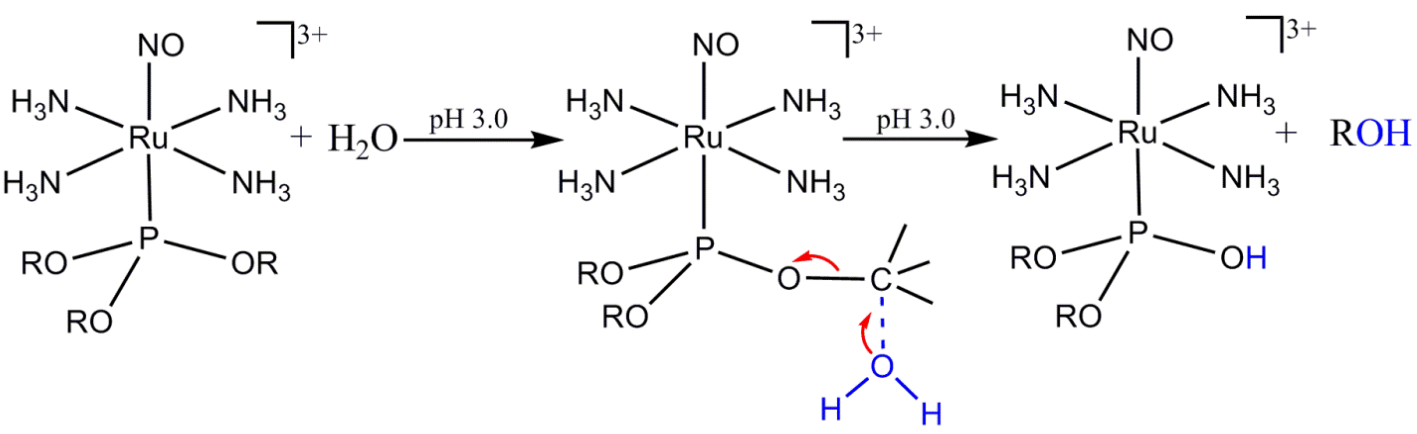

(b)

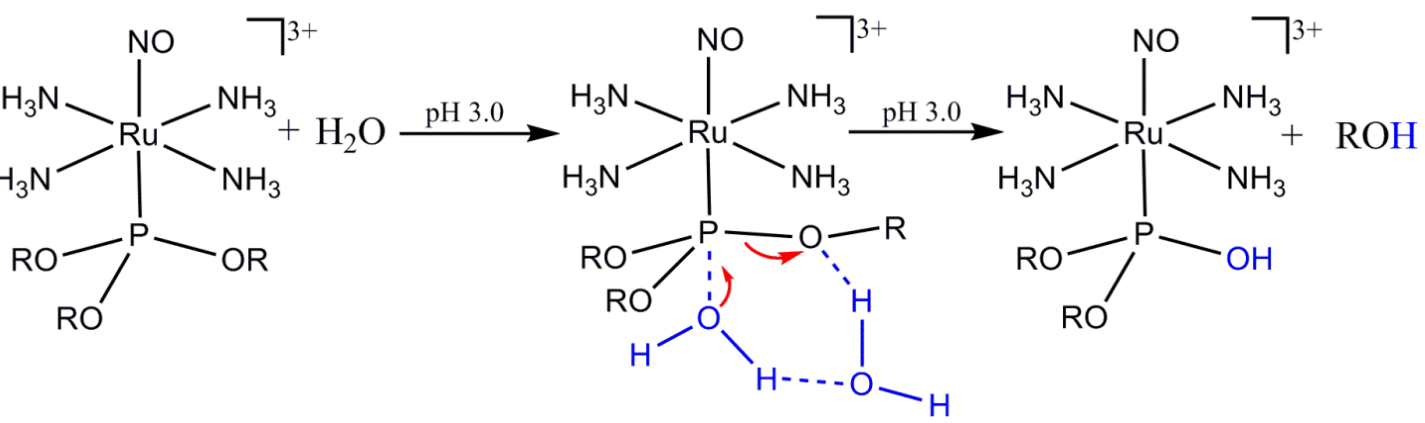

\subsubsection{Propriedades eletroquímicas e $\mathrm{k}_{\text {-NO }}$ de nitrosilos complexos de fósforo(III)}

A atividade biológica dos nitrosilos complexos de rutênio está diretamente relacionada à sua habilidade de liberar óxido nítrico e/ou nitroxila, após a redução por um (reação 23) e/ou dois elétrons (reação 24), respectivamente [27; 52]. Recentemente foi descrito que o complexo trans- $\left[\mathrm{Ru}(\mathrm{NO})\left(\mathrm{NH}_{3}\right)_{4} \mathrm{P}\left(\mathrm{OC}_{2} \mathrm{H}_{5}\right)_{3}\right]^{3+}$ pode gerar $\mathrm{NO}$ e/ou $\mathrm{HNO}$ dependendo das condições de redução do meio $\left(\mathrm{E}_{\mathrm{NO}+/ \mathrm{NO}}=-0,10 \mathrm{~V}\right.$ e $\mathrm{E}_{\mathrm{NO} / \mathrm{NO}-}=-0,70 \mathrm{~V}$ vs. $\left.\mathrm{ECS}\right)$ [52]. 


$$
\begin{aligned}
& \text { trans }-\left[\mathrm{Ru}(\mathrm{NO})\left(\mathrm{NH}_{3}\right)_{4} \mathrm{P}(\mathrm{OR})_{3}\right]^{3+} \frac{+\mathrm{e}^{-}}{-\mathrm{e}^{-}} \text {trans- }\left[\mathrm{Ru}(\mathrm{NO})\left(\mathrm{NH}_{3}\right)_{4} \mathrm{P}(\mathrm{OR})_{3}\right]^{2+} \\
& \text { trans }-\left[\mathrm{Ru}(\mathrm{NO})\left(\mathrm{NH}_{3}\right)_{4} \mathrm{P}(\mathrm{OR})_{3}\right]^{2+} \frac{+\mathrm{e}^{-}}{\stackrel{-\mathrm{e}^{-}}{\longrightarrow}} \text { trans }-\left[\mathrm{Ru}\left(\mathrm{NO}^{-}\right)\left(\mathrm{NH}_{3}\right)_{4} \mathrm{P}(\mathrm{OR})_{3}\right]^{+}
\end{aligned}
$$

Esta mesma habilidade foi investigada para íons trans- $\left[\mathrm{Ru}(\mathrm{NO})\left(\mathrm{NH}_{3}\right)_{4} \mathrm{P}(\mathrm{OR})_{3}\right]^{3+}$ aqui em estudo, sendo que todos apresentaram um perfil de voltamograma cíclico semelhante em meio aquoso. Como exemplo, a Figura 24 a mostra o voltamograma cíclico para o íon trans- $\left[\mathrm{Ru}(\mathrm{NO})\left(\mathrm{NH}_{3}\right)_{4}\left(\mathrm{P}\left(\mathrm{OCH}_{3}\right)_{3}\right)\right]^{3+}$. Na Figura 24 b, o voltamograma de pulso diferencial evidencia melhor os processos de redução $\left[\mathrm{Ru}\left(\mathrm{NO}^{+}\right)\right] /[\mathrm{Ru}(\mathrm{NO})]$ e $[\mathrm{Ru}(\mathrm{NO})] /\left[\mathrm{Ru}\left(\mathrm{NO}^{-}\right)\right]$. Os valores de potencial pico destes processos de redução são apresentados na Tabela 8.

Figura 24 - trans- $\left[\mathrm{Ru}(\mathrm{NO})\left(\mathrm{NH}_{3}\right)_{4}\left(\mathrm{P}\left(\mathrm{OCH}_{3}\right)_{3}\right)\right]^{3+}$ em meio aquoso $\mathrm{pH} 2,0, \mu=0,1 \mathrm{~mol} \mathrm{~L}^{-1}$, $\mathrm{C}_{\mathrm{Ru}}=1,5 \times 10^{-3} \mathrm{~mol} \mathrm{~L}^{-1}, \mathrm{~T}=5 \pm 0,1^{\circ} \mathrm{C}$ (a) Voltamograma cíclico, $\mathrm{v}=100 \mathrm{mV} \mathrm{s}^{-1}$; (b) Voltamograma de pulso diferencial, $\mathrm{v}=20 \mathrm{mV} \mathrm{s}^{-1}$.

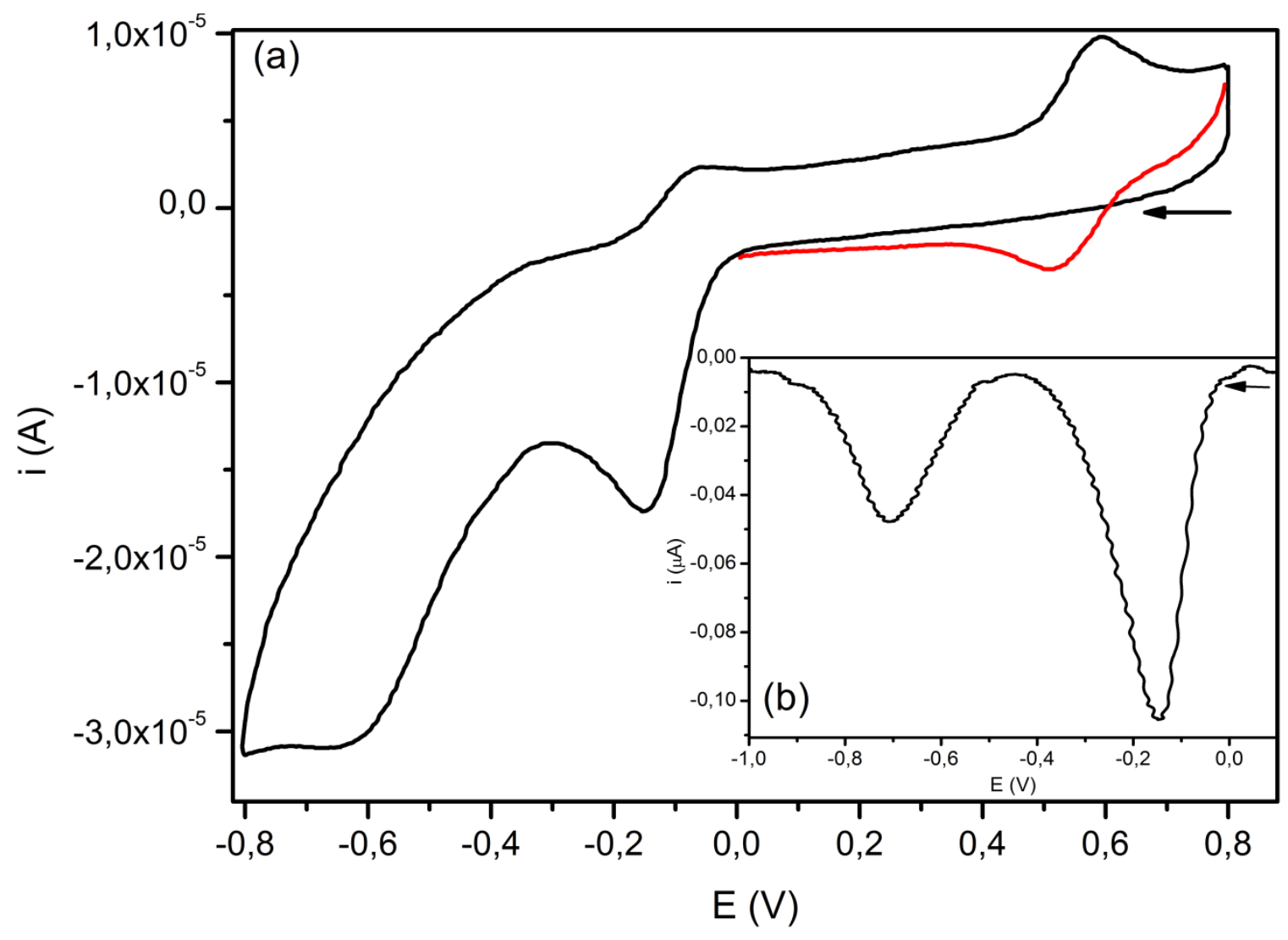


De acordo com as constantes de velocidade de liberação específica de NO ( $\left.\mathrm{k}_{-\mathrm{NO}}\right)$ calculadas para a série de complexos trans-[Ru(NO)(NH$\left.)_{4} \mathrm{~L}\right]^{\mathrm{n}+}[27 ; 46]$, o valor de $\mathrm{k}_{-\mathrm{NO}}$ está associado ao efeito trans apresentado pelo ligante em posição trans ao NO. Os ésteres de fósforo(III) são conhecidos por exibirem elevado efeito e influência trans [46]. Dessa forma, o k-NO (reação 25) foi calculado para a série trans- $\left[\mathrm{Ru}(\mathrm{NO})\left(\mathrm{NH}_{3}\right)_{4} \mathrm{P}(\mathrm{OR})_{3}\right]^{3+}$ a fim de observar como as modificações em $\mathrm{R}$ podem influenciar nos valores de $\mathrm{k}_{\text {-NO }}$ (Tabela 8).

$$
\text { trans- }\left[\mathrm{Ru}(\mathrm{NO})\left(\mathrm{NH}_{3}\right)_{4} \mathrm{P}(\mathrm{OR})_{3}\right]^{2+} \stackrel{\mathrm{k}_{-\mathrm{NO}}}{\longrightarrow} \text { trans- }\left[\mathrm{Ru}\left(\mathrm{H}_{2} \mathrm{O}\right)\left(\mathrm{NH}_{3}\right)_{4} \mathrm{P}(\mathrm{OR})_{3}\right]^{2+}+\mathrm{NO}
$$

Tabela 8 - Dados eletroquímicos e $\mathrm{k}_{-\mathrm{NO}}$ para íons complexos do tipo trans-[Ru(NO) $\left.\left(\mathrm{NH}_{3}\right)_{4} \mathrm{P}(\mathrm{III})\right]^{3+}$ em pH 2,0 e $25,0 \pm 0,1^{\circ} \mathrm{C}$

\begin{tabular}{lccc}
\hline \multicolumn{1}{c}{ Complexos } & $\mathbf{E}_{\mathrm{NO}+/ \mathbf{N O}^{*}}$ & $\mathbf{E}_{\mathrm{NO} / \mathrm{NO}^{-}}{ }^{*}$ & $\mathbf{k}_{-\mathbf{N O}}$ \\
\hline trans $-\left[\mathrm{Ru}(\mathrm{NO})\left(\mathrm{NH}_{3}\right)_{4}\left(\mathrm{P}\left(\mathrm{OCH}_{3}\right)_{3}\right)\right]^{3+}$ & $-0,12$ & $-0,68$ & 0,26 \\
trans $-\left[\mathrm{Ru}(\mathrm{NO})\left(\mathrm{NH}_{3}\right)_{4}\left(\mathrm{P}\left(\mathrm{OC}_{2} \mathrm{H}_{5}\right)_{3}\right)\right]^{3+}$ & $-0,10[51]$ & $-0,70[52]$ & $0,97[51]$ \\
trans $-\left[\mathrm{Ru}(\mathrm{NO})\left(\mathrm{NH}_{3}\right)_{4}\left(\mathrm{P}\left(\mathrm{O}^{\mathrm{i}} \mathrm{C}_{3} \mathrm{H}_{7}\right)_{3}\right)\right]^{3+}$ & $-0,19$ & $-0,39$ & 2,85 \\
trans $-\left[\mathrm{Ru}(\mathrm{NO})\left(\mathrm{NH}_{3}\right)_{4}\left(\mathrm{P}\left(\mathrm{OC}_{4} \mathrm{H}_{9}\right)_{3}\right)\right]^{3+}$ & $-0,10$ & $-0,47$ & 0,91 \\
trans- $\left[\mathrm{Ru}(\mathrm{NO})\left(\mathrm{NH}_{3}\right)_{4}\left(\mathrm{P}(\mathrm{O})(\mathrm{OH})_{2}\right)\right]^{2+}$ & $-0,52[59]$ & $-0,79$ & 0,22 \\
trans- $\left[\mathrm{Ru}(\mathrm{NO})\left(\mathrm{NH}_{3}\right)_{4}\left(\mathrm{P}(\mathrm{O})\left(\mathrm{OC}_{2} \mathrm{H}_{5}\right)_{2}\right)\right]^{2+}$ & $-0,50$ & $-0,80$ & 0,24 \\
\hline
\end{tabular}

* Valores $v s$. ECS

Apesar de o valor de k-No para o íon complexo trans-[Ru(NO) $\left.\left(\mathrm{NH}_{3}\right)_{4}\left(\mathrm{P}_{(\mathrm{OC}}\left(\mathrm{OC}_{5}\right)_{3}\right)\right]^{3+}$ ter sido anteriormente medido em $\mathrm{pH}$ 5,0, neste trabalho, para efeito de comparação, optou-se por realizar tal calculo em $\mathrm{pH}$ 2,0 em decorrência, principalmente, da baixa estabilidade do nitrosilo complexo de ácido fosforoso (conforme discutido na seção 4.1). Assim, o valor de $\mathrm{k}_{\text {-NO }}$ para o nitrosilo complexo de trietil fosfito foi reavaliado em $\mathrm{pH}$ 2,0 sendo observado que o valor $\mathrm{k}_{-\mathrm{NO}}$ não apresenta modificação em função do $\mathrm{pH}\left(\mathrm{k}_{-\mathrm{NO}}=0,97 \mathrm{~s}^{-1} \mathrm{em} \mathrm{pH} 2,0\right.$ $\mathrm{e}_{-\mathrm{NO}}=0,98 \mathrm{~s}^{-1} \mathrm{em} \mathrm{pH} \mathrm{5,0} \mathrm{[51]).}$ 
Os valores de constante de liberação específica de $\mathrm{NO}\left(\mathrm{k}_{-\mathrm{NO}}\right)$ em meio aquoso decresce na sequência: $\mathrm{P}\left(\mathrm{O}^{\mathrm{i}} \mathrm{C}_{3} \mathrm{H}_{7}\right)_{3}>\mathrm{P}\left(\mathrm{OC}_{2} \mathrm{H}_{5}\right)_{3}>\mathrm{P}\left(\mathrm{OC}_{4} \mathrm{H}_{9}\right)_{3}>\mathrm{P}\left(\mathrm{OCH}_{3}\right)_{3}>\mathrm{P}(\mathrm{O})\left(\mathrm{OC}_{2} \mathrm{H}_{5}\right)_{2}>$ $\mathrm{P}(\mathrm{O})(\mathrm{OH})_{2}$. Esta é exatamente a mesma sequência observada para a reação de aquação da pirazina (pz) nos íons trans- $\left[\mathrm{Ru}(\mathrm{pz})\left(\mathrm{NH}_{3}\right)_{4} \mathrm{P}(\mathrm{III})\right]^{\mathrm{n}+}[54 ; 105]$. Em termos gerais, pode-se afirmar que o aumento de grupos $\sigma$-indutores ligados ao carbono $\alpha$ do fosfito aumenta consideravelmente o efeito trans labilizante exercido pelo éster de fósforo. Ao mesmo tempo, a retirada de grupos $\sigma$-indutores ligados ao átomo de oxigênio da estrutura do fosfito, como ocorre no caso do dietil fosfito e do ácido fosforoso, reduz a capacidade trans labilizante dos mesmos.

Mesmo utilizando temperatura de $5^{\circ} \mathrm{C}$ e velocidades de varredura de até $2 \mathrm{~V} \mathrm{~s}^{-1}$, o processo de oxidação $\mathrm{NO}^{`} / \mathrm{NO}$ não foi claramente observado para os complexos em estudo neste trabalho. Assim cálculo de $\mathrm{k}_{-\mathrm{NO}}$ - por meio do método de Nicholson e Shain [77] não foi possível. A difícil observação do processo de oxidação NO/NO indica que, para esta série de nitrosilos complexos, $\mathrm{k}_{-\mathrm{NO}^{-}}>>\mathrm{k}_{-\mathrm{NO}}$. 


\subsection{Caracterização do íon complexo trans- $\left[\mathrm{Ru}(\mathrm{CO})\left(\mathrm{NH}_{3}\right)_{4}\left(\mathrm{P}(\mathrm{OH})_{3}\right)\right]^{2+}$ e sua estabilidade em meio aquoso}

\subsubsection{Caracterização do complexo trans $-\left[\mathrm{Ru}(\mathrm{CO})\left(\mathrm{NH}_{3}\right)_{4}\left(\mathrm{P}(\mathrm{O})(\mathrm{OH})_{2}\right)\right](\mathrm{X})$ em que} $\mathrm{X}=\mathrm{ZnCl}_{4}{ }^{2-}$ ou $\mathrm{Cl}^{-}$

A síntese do carbonilo complexo de ácido fosforoso deu origem a um sólido amarelo bem claro. Os resultados de Análise Elementar de carbono, nitrogênio e hidrogênio e de Espectrometria de Emissão por Plasma de rutênio para o sólido encontram-se na Tabela 9 e concordam com as porcentagens teóricas calculadas para este complexo.

Tabela 9 - Dados de Análise Elementar e Espectrometria de Emissão por Plasma para o trans- $\left[\mathrm{Ru}(\mathrm{CO})\left(\mathrm{NH}_{3}\right)_{4}\left(\mathrm{P}(\mathrm{O})(\mathrm{OH})_{2}\right)\right](\mathrm{Cl})$

\begin{tabular}{cccc}
\hline & Teórico $(\%)$ & Experimental $(\%)$ & Erro (\%) \\
\hline \multicolumn{4}{c}{ trans $-\left[\mathrm{Ru}(\mathrm{CO})\left(\mathrm{NH}_{3}\right)_{4}\left(\mathrm{P}(\mathrm{O})(\mathrm{OH})_{2}\right)\right](\mathrm{Cl})$} \\
\hline $\mathbf{C}$ & 3,82 & 3,84 & 0,5 \\
$\mathbf{H}$ & 4,81 & 4,70 & 2,2 \\
$\mathbf{N}$ & 17,81 & 17,56 & 1,4 \\
$\mathbf{R u}$ & 32,12 & 31,20 & 2,8
\end{tabular}

\subsubsection{Difração de Raio-X: trans-[Ru(CO) $\left.\left(\mathrm{NH}_{3}\right)_{4}\left(\mathrm{P}(\mathrm{OH})_{3}\right)\right]\left(\mathrm{ZnCl}_{4}\right) \cdot 3 \mathrm{H}_{2} \mathrm{O}$}

A estrutura cristalina deste complexo, a semelhança do descrito para o trans- $\left[\mathrm{Ru}(\mathrm{NO})\left(\mathrm{NH}_{3}\right)_{4}\left(\mathrm{P}(\mathrm{O})(\mathrm{OH})_{2}\right)\right] \mathrm{ZnCl}_{4}, \quad$ apresenta $\quad$ o cátion octaédrico trans- $\left[\mathrm{Ru}(\mathrm{CO})\left(\mathrm{NH}_{3}\right)_{4}\left(\mathrm{P}(\mathrm{OH})_{3}\right)\right]^{2+}$ e como contra íon o ânion $\left[\mathrm{ZnCl}_{4}\right]^{2^{-}}$(Figura 25). No cátion, os quatro fragmentos $\mathrm{NH}_{3}$ mantém posição equatorial e os grupos $\left[\mathrm{H}_{3} \mathrm{PO}_{3}\right]$ e $\mathrm{CO}$ estão trans-posicionados na direção axial. As distâncias e ângulos de ligação são apresentados na Tabela 11. 
Tabela 10 - Dados cristalográficos para o trans- $\left[\mathrm{Ru}(\mathrm{CO})\left(\mathrm{NH}_{3}\right)_{4}\left(\mathrm{P}(\mathrm{OH})_{3}\right)\right]\left(\mathrm{ZnCl}_{4}\right) \cdot \mathrm{H}_{2} \mathrm{O}$

\begin{tabular}{ll}
\hline Fórmula & trans- $\left[\mathrm{Ru}(\mathrm{CO})\left(\mathrm{NH}_{3}\right)_{4}\left(\mathrm{P}(\mathrm{OH})_{3}\right)\right]\left(\mathrm{ZnCl}_{4}\right) \cdot \mathrm{H}_{2} \mathrm{O}$ \\
\hline Grupo espacial & Monoclínico, $\mathrm{P}_{21} / \mathrm{c}$ \\
$a(\AA)$ & $8,8478(3)$ \\
$b(\AA)$ & $7,3811(2)$ \\
$c(\AA)$ & $25,0130(8)$ \\
$\beta($ deg $)$ & $93,998(2)$ \\
$Z$ & 4 \\
$V\left(\AA^{3}\right)$ & $1629,54(9)$ \\
$\lambda\left(\mathrm{Mo} \mathrm{K \alpha}^{-}(\AA)\right.$ & 0,71073 \\
$\rho\left(\mathrm{mg} \mathrm{m}^{-3}\right)$ & 2,056 \\
$\mathrm{R}$ índices & $\mathrm{R} 1=0,0432, \mathrm{wR} 2=0,0906$ \\
\hline
\end{tabular}

Figura 25 - Representação ORTEP-3 da estrutura trans- $\left[\mathrm{Ru}(\mathrm{CO})\left(\mathrm{NH}_{3}\right)_{4}\left(\mathrm{P}(\mathrm{OH})_{3}\right)\right]\left(\mathrm{ZnCl}_{4}\right) \cdot \mathrm{H}_{2} \mathrm{O}$ com elipsoides de deslocamentos que apresentam probabilidades de $40 \%$
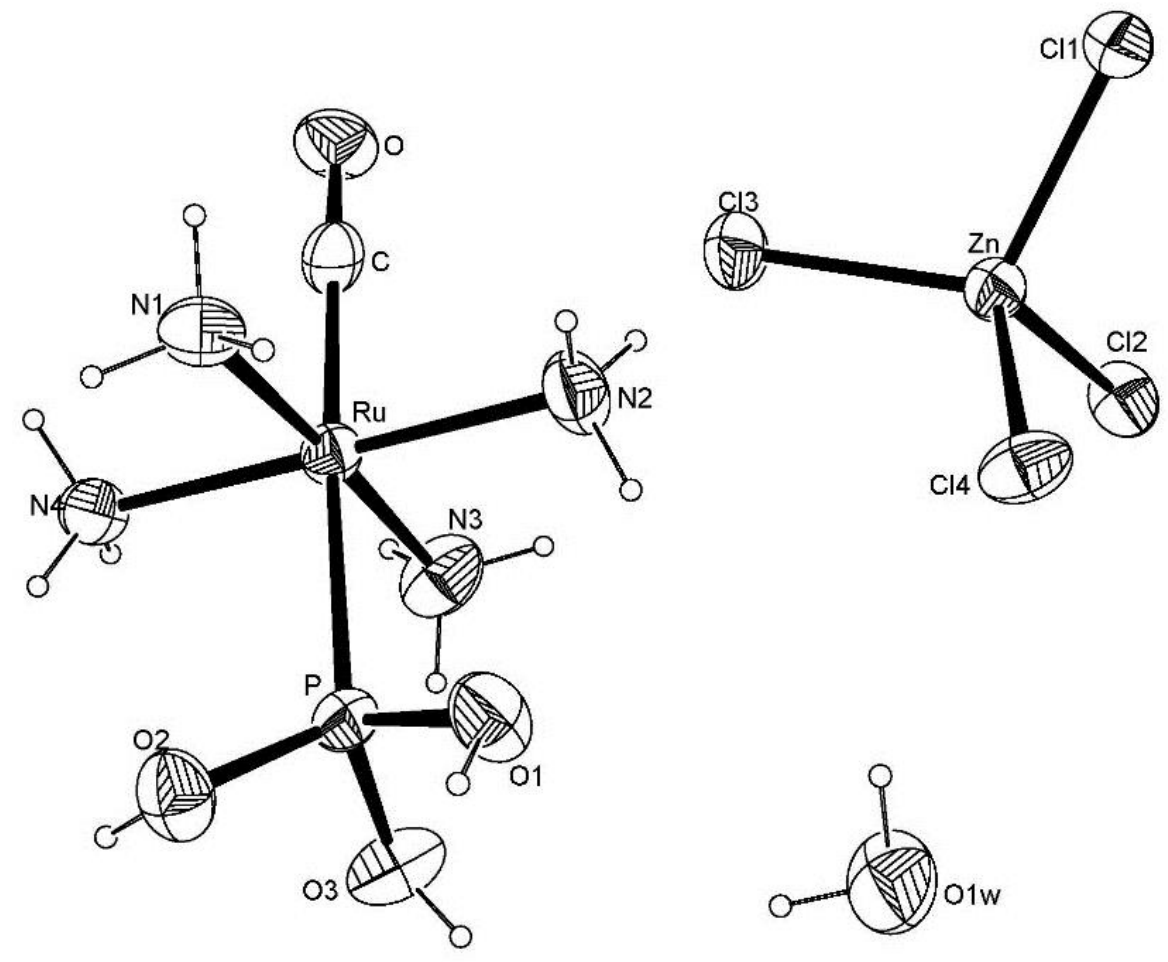
Tabela 11 - Distâncias e ângulos de ligação para o complexo trans- $\left[\mathrm{Ru}(\mathrm{CO})\left(\mathrm{NH}_{3}\right)_{4}\left(\mathrm{P}(\mathrm{OH})_{3}\right)\right]\left(\mathrm{ZnCl}_{4}\right) \cdot \mathrm{H}_{2} \mathrm{O}$

\begin{tabular}{cccc}
\hline \multicolumn{5}{c}{ Distâncias de ligação $(\AA)$} \\
Ru-C & $1,882(6)$ & $\mathrm{P}-\mathrm{O}(2)$ & $1,588(4)$ \\
$\mathrm{Ru}-\mathrm{P}$ & $2,3763(15)$ & $\mathrm{P}-\mathrm{O}(3)$ & $1,577(4)$ \\
$\mathrm{P}-\mathrm{O}(1)$ & $1,569(4)$ & $\mathrm{C}-\mathrm{O}$ & $1,150(6)$ \\
\hline \multicolumn{4}{c}{ Ângulos de ligação $\left(^{\circ}\right)$} \\
$\mathrm{C}-\mathrm{Ru}-\mathrm{N}(1)$ & $93,3(2)$ & $\mathrm{N}(3)-\mathrm{Ru}-\mathrm{N}(2)$ & $91,8(2)$ \\
$\mathrm{C}-\mathrm{Ru}-\mathrm{N}(2)$ & $91,1(2)$ & $\mathrm{C}-\mathrm{Ru}-\mathrm{P}$ & $176,94(18)$ \\
$\mathrm{C}-\mathrm{Ru}-\mathrm{N}(3)$ & $89,5(2)$ & $\mathrm{O}(1)-\mathrm{P}-\mathrm{O}(3)$ & $107,6(3)$ \\
$\mathrm{C}-\mathrm{Ru}-\mathrm{N}(4)$ & $89,6(2)$ & $\mathrm{O}(1)-\mathrm{P}-\mathrm{O}(2)$ & $99,6(2)$ \\
$\mathrm{N}(2)-\mathrm{Ru}-\mathrm{N}(1)$ & $89,7(2)$ & $\mathrm{O}(3)-\mathrm{P}-\mathrm{O}(2)$ & $105,7(2)$ \\
$\mathrm{N}(3)-\mathrm{Ru}-\mathrm{N}(1)$ & $176,77(19)$ & $\mathrm{O}(1)-\mathrm{P}-\mathrm{Ru}$ & $111,36(17)$ \\
$\mathrm{N}(4)-\mathrm{Ru}-\mathrm{N}(1)$ & $88,78(19)$ & $\mathrm{O}(2)-\mathrm{P}-\mathrm{Ru}$ & $120,60(16)$ \\
$\mathrm{N}(3)-\mathrm{Ru}-\mathrm{N}(4)$ & $89,65(19)$ & $\mathrm{O}(3)-\mathrm{P}-\mathrm{Ru}$ & $110,88(17)$ \\
$\mathrm{N}(2)-\mathrm{Ru}-\mathrm{N}(4)$ & $178,32(19)$ & $\mathrm{O}-\mathrm{C}-\mathrm{Ru}$ & $178,9(5)$ \\
\hline
\end{tabular}

Comparando os dados geométricos do trans- $\left[\mathrm{Ru}(\mathrm{NO})\left(\mathrm{NH}_{3}\right)_{4}\left(\mathrm{P}(\mathrm{O})(\mathrm{OH})_{2}\right)\right] \mathrm{ZnCl}_{4}$, com do trans- $\left[\mathrm{Ru}(\mathrm{CO})\left(\mathrm{NH}_{3}\right)_{4}\left(\mathrm{P}(\mathrm{OH})_{3}\right)\right]\left(\mathrm{ZnCl}_{4}\right) \cdot \mathrm{H}_{2} \mathrm{O}$ observa-se que a distância de ligação $\mathrm{Ru}-\mathrm{P}$ é reduzida de 2,4136 para 2,3763. Isto provavelmente se deva à menor competição pelos elétrons $\pi$ existente no eixo P-Ru-CO que no eixo P-Ru-NO, o que está de acordo com a maior acidez $\pi$ do ligante nitrosônio em relação ao carbonil. O ângulo Ru-NO observado é de 172,8 enquanto que o Ru-CO é de 178,9, esta maior proximidade da linearidade pode ser atribuída ao fato do ácido fosforoso estar protonado no carbonilo complexo, enquanto que no nitrosilo complexo este está desprotonado.

\subsubsection{Espectroscopia Eletrônica}

O espectro eletrônico do íon trans- $\left[\mathrm{Ru}(\mathrm{CO})\left(\mathrm{NH}_{3}\right)_{4}\left(\mathrm{P}(\mathrm{OH})_{3}\right)\right]^{2+}$ é apresentado na Figura 26 sendo composto por três bandas em: $320 \mathrm{~nm}\left(\varepsilon \sim 63 \mathrm{~L} \mathrm{~mol}^{-1} \mathrm{~cm}^{-1}\right), 280 \mathrm{~nm}$ 
$\left(\varepsilon=375 \mathrm{~L} \mathrm{~mol}^{-1} \mathrm{~cm}^{-1}\right)$ e $221 \mathrm{~nm}\left(\varepsilon=2522 \mathrm{~L} \mathrm{~mol}^{-1} \mathrm{~cm}^{-1}\right)$. Este espectro apresenta perfil similar ao observado para o complexo trans- $\left[\mathrm{Ru}(\mathrm{CO})\left(\mathrm{NH}_{3}\right)_{4}\left(\mathrm{P}\left(\mathrm{OCH}_{2} \mathrm{CH}_{3}\right)_{3}\right)\right]^{2+}[53 ; 106]$.

De acordo com os cálculos de TD-DFT, a banda em $320 \mathrm{~nm}$ trata-se essencialmente de uma do tipo transição d-d HOMO-1,2( $\left.\mathrm{d}_{\mathrm{xz}} \mathrm{d}_{\mathrm{yz}} \mathrm{Ru}\right) \rightarrow \mathrm{LUMO}+0,1\left(\mathrm{~d}_{\mathrm{x} 2} \mathrm{y}^{2} \mathrm{~d}_{\mathrm{z}} \mathrm{Ru}\right)$. A banda em 280 nm apresenta uma maior composição da transição do tipo d-d HOMO-1,2( $\left.d_{x z} d_{y z} R u\right) \rightarrow$ LUMO+0,1 $\left(d_{x} 2 y_{2} d_{z} 2 R u\right)$ e uma menor contribuição da TCML $\left(\mathrm{HOMO}\left(\mathrm{d}_{\mathrm{xy}} \mathrm{Ru}\right) \rightarrow \mathrm{LUMO}+2,3(\pi * \mathrm{CO})\right)$. A banda em $221 \mathrm{~nm}$ é atribuída a TCML $\left(\mathrm{HOMO}\left(\mathrm{d}_{\mathrm{xy}} \mathrm{Ru}\right) \rightarrow \mathrm{LUMO}+2,3(\pi * \mathrm{CO})\right)$.

Figura 26 - Espectro eletrônico do íon trans- $\left[\mathrm{Ru}(\mathrm{CO})\left(\mathrm{NH}_{3}\right)_{4}\left(\mathrm{P}(\mathrm{OH})_{3}\right)\right]^{2+}$ em solução pH 2,0, $\mu=0,1 \mathrm{~mol} \mathrm{~L}^{-1} \mathrm{CF}_{3} \mathrm{COONa}$, temperatura de $25 \pm 0,1^{\circ} \mathrm{C}$ e cubeta de $1,0 \mathrm{~cm}$ de caminho ótico, (a) $\mathrm{C}_{\mathrm{Ru}}=4,0 \times 10^{-4} \mathrm{~mol} \mathrm{~L}^{-1}$ e (b) $\mathrm{C}_{\mathrm{Ru}}=1,0 \times 10^{-3} \mathrm{~mol} \mathrm{~L}^{-1}$

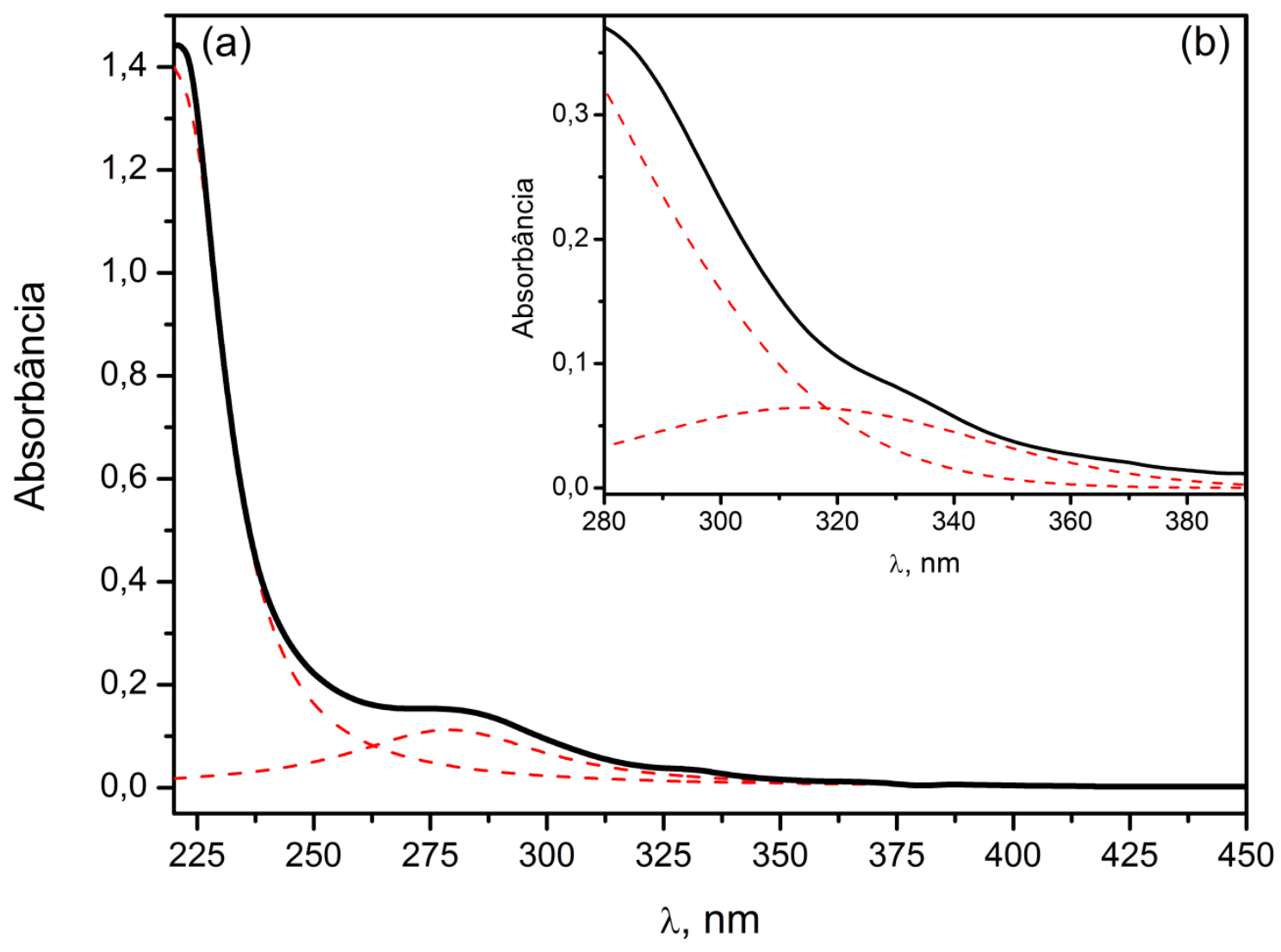




\subsubsection{Voltametria Cíclica}

$\mathrm{O}$ voltamograma cíclico da solução contendo o íon trans- $\left[\mathrm{Ru}(\mathrm{CO})\left(\mathrm{NH}_{3}\right)_{4}\left(\mathrm{P}(\mathrm{OH})_{3}\right)\right]^{2+}$ (Figura 27) apresenta um pico de potencial anódico $\left(\mathrm{E}_{\mathrm{pa}}\right)$ em $0,76 \mathrm{~V}$ vs ECS referente a oxidação do $\mathrm{CO}$ a $\mathrm{CO}_{2}$ (reação 26). Após essa oxidação o aquo complexo é formado e o par $\left[\mathrm{Ru}^{\mathrm{III}}\left(\mathrm{OH}_{2}\right)\right] /\left[\mathrm{Ru}^{\mathrm{II}}\left(\mathrm{OH}_{2}\right)\right]$ é observado em $0,30 \mathrm{~V}$ vs ECS (reação 27).

$$
\begin{aligned}
& \text { trans- }\left[\mathrm{Ru}(\mathrm{CO})\left(\mathrm{NH}_{3}\right)_{4} \mathrm{P}(\mathrm{OH})_{3}\right]^{2+}+\mathrm{H}_{2} \mathrm{O} \stackrel{-2 \mathrm{e}^{-}}{\longrightarrow} \text { trans- }\left[\mathrm{Ru}\left(\mathrm{H}_{2} \mathrm{O}\right)\left(\mathrm{NH}_{3}\right)_{4} \mathrm{P}(\mathrm{OH})_{3}\right]^{2+}+\mathrm{CO}_{2} \\
& \text { trans }-\left[\mathrm{Ru}\left(\mathrm{H}_{2} \mathrm{O}\right)\left(\mathrm{NH}_{3}\right)_{4} \mathrm{P}(\mathrm{OH})_{3}\right]^{3+} \stackrel{+\mathrm{e}^{-}}{\rightleftharpoons} \text { trans }-\left[\mathrm{Ru}\left(\mathrm{e}_{2} \mathrm{O}\right)\left(\mathrm{NH}_{3}\right)_{4} \mathrm{P}(\mathrm{OH})_{3}\right]^{2+}
\end{aligned}
$$

Figura 27 - Voltamograma cíclico do composto trans- $\left[\mathrm{Ru}(\mathrm{CO})\left(\mathrm{NH}_{3}\right)_{4}\left(\mathrm{P}(\mathrm{O})(\mathrm{OH})_{2}\right)\right](\mathrm{Cl})$ em solução pH 2,0, $\mu=0,1 \mathrm{molL}^{-1} \mathrm{CF}_{3} \mathrm{COONa}, \mathrm{v}=50 \mathrm{mVs}^{-1}$ e $\mathrm{C}_{\mathrm{Ru}}=1,0 \times 10^{-3} \mathrm{~mol} \mathrm{~L}^{-1}, 25 \pm$ $0,1^{\circ} \mathrm{C},(-)$ primeiro ciclo (-) segundo ciclo

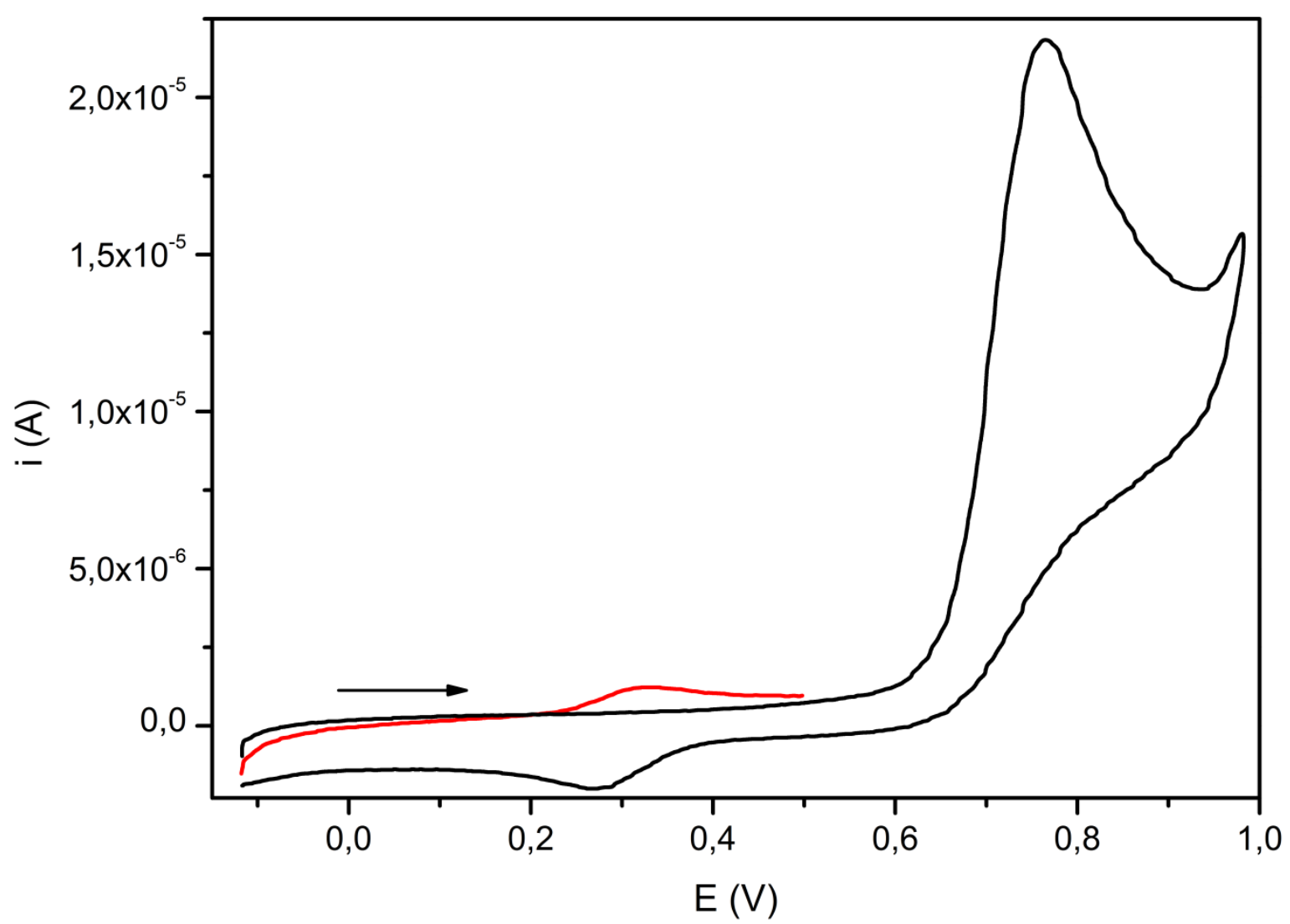


À solução contendo trans- $\left[\mathrm{Ru}(\mathrm{CO})\left(\mathrm{NH}_{3}\right)_{4}\left(\mathrm{P}(\mathrm{OH})_{3}\right)\right]^{2+}$ foi adicionado o complexo $\left[\mathrm{Ru}\left(\mathrm{NH}_{3}\right)_{6}\right](\mathrm{Cl})_{3}$, o que permitiu estimar o número de elétrons envolvido no processo de oxidação do monóxido de carbono como igual a 2,2. Isto corrobora para a oxidação do fragmento $\mathrm{CO}$ a $\mathrm{CO}_{2}$ (reação 26).

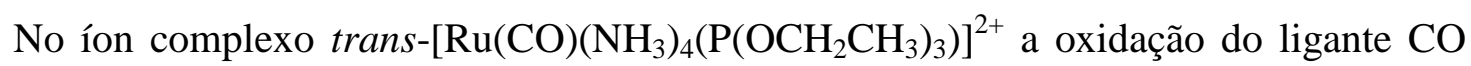
ocorre em $\mathrm{E}_{\mathrm{pa}}=1,11 \mathrm{~V} v s \mathrm{ECS}$, ou seja, um valor $0,35 \mathrm{~V}$ mais positivo em relação ao carbonilo de ácido fosforoso.

\subsubsection{Espectroscopia Vibracional.}

A Figura 28 ilustra o espectro vibracional em estado sólido do complexo trans- $\left[\mathrm{Ru}(\mathrm{CO})\left(\mathrm{NH}_{3}\right)_{4}\left(\mathrm{P}(\mathrm{O})(\mathrm{OH})_{2}\right)\right](\mathrm{Cl})$. As principais bandas e respectivas atribuições são mostradas na Tabela 12.

Figura 28 - Espectro na região do infravermelho do composto trans- $\left[\mathrm{Ru}(\mathrm{CO})\left(\mathrm{NH}_{3}\right)_{4}\left(\mathrm{P}(\mathrm{O})(\mathrm{OH})_{2}\right)\right](\mathrm{Cl})$ em pastilha de $\mathrm{KBr}$

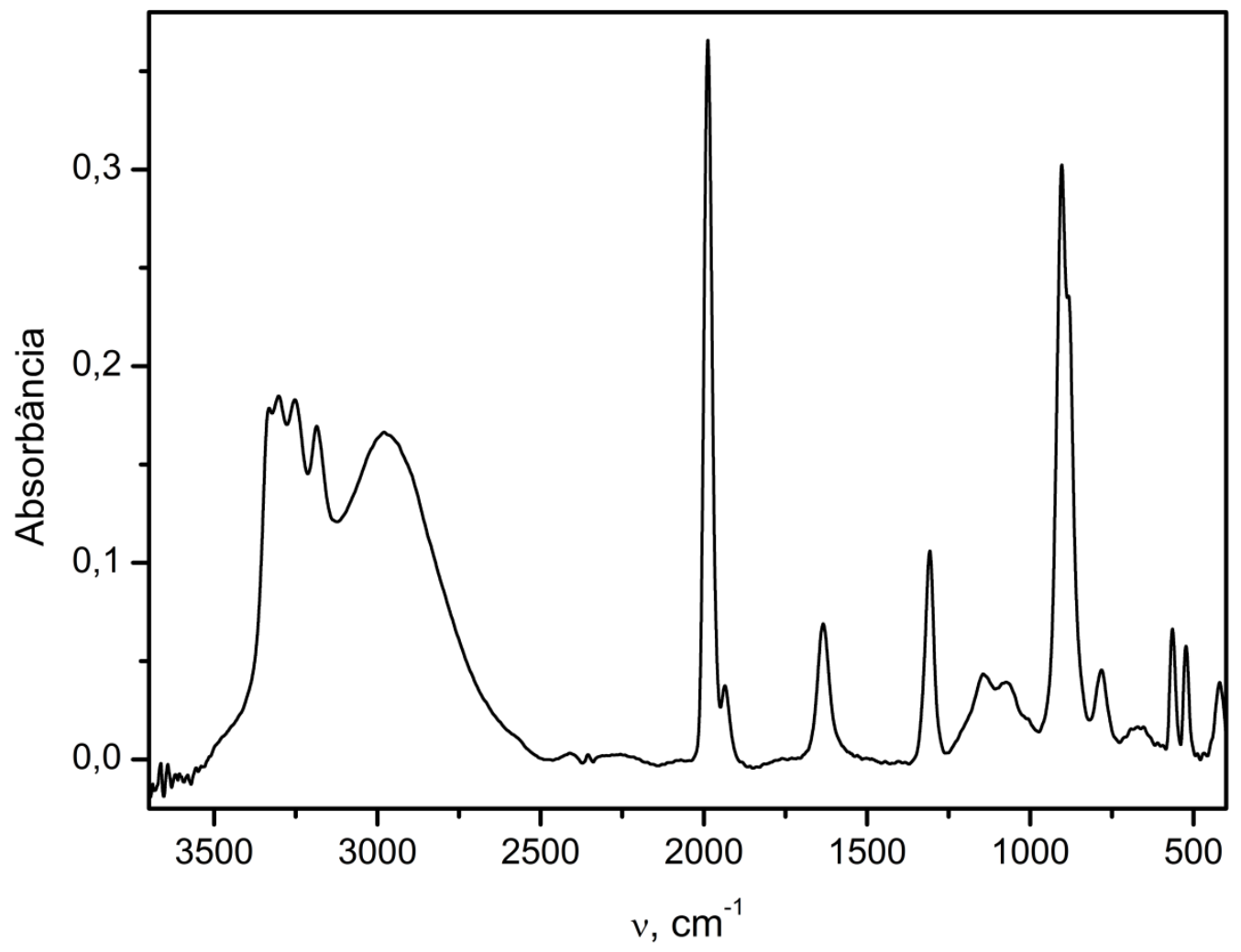


As duas frequências de estiramentos observadas para o grupo $\mathrm{CO}$ devem-se ao fato do ligante ácido fosforoso estar tanto em sua forma protonada quando desprotonada. Assim, a

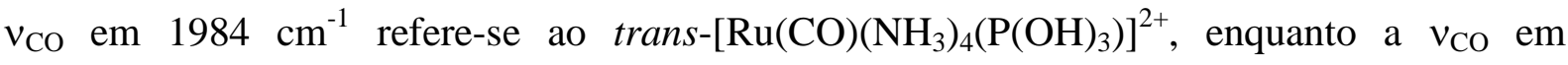
$1932 \mathrm{~cm}^{-1}$ refere-se ao trans- $\left[\mathrm{Ru}(\mathrm{CO})\left(\mathrm{NH}_{3}\right)_{4}\left(\mathrm{P}(\mathrm{O})(\mathrm{OH})_{2}\right)\right]^{+}$.

Tabela 12 - Principais bandas e as atribuições propostas

\begin{tabular}{cc}
\hline Número de onda $\left(\mathbf{c m}^{-1}\right)^{*}$ & Atribuições \\
\hline 3300,3252 e $3183 \mathrm{~m}$ & $v_{\mathrm{NH}}$ \\
$2974 \mathrm{~m}$ & $v_{\mathrm{OH}}$ \\
$1984 \mathrm{~F}$ e $1932 \mathrm{f}$ & $v_{\mathrm{CO}}$ \\
$902 \mathrm{~F}$ e $882 \mathrm{~m}$ & $v_{\mathrm{PO} \text { ou POH }}$ \\
$1103 \mathrm{~m}$ & $v_{\mathrm{P}-\mathrm{O}-\mathrm{H}}$ \\
$1630 \mathrm{~m}$ & $\delta_{\mathrm{asNH}}$ \\
$1307 \mathrm{~m}$ & $\delta_{\mathrm{sNH}}$ \\
560 e $520 \mathrm{f}$ & $\delta_{\mathrm{Ru}-\mathrm{CO}}$ \\
\hline
\end{tabular}

* Pastilha de $\mathrm{KBr}$; resolução de $\pm 4 \mathrm{~cm}^{-1}$; Intensidades: $\mathrm{F}=$ forte, $\mathrm{m}=$ médio e $\mathrm{f}=$ fraca.

A frequência de estiramento do grupo $\mathrm{CO}$ em complexos do tipo trans-[Ru(CO) $\left.\left(\mathrm{NH}_{3}\right)_{4} \mathrm{~L}\right]^{2+}$ pode ser associada com o grau de densidade eletrônica transferida ao orbital $\pi^{*}$ do CO. Assim, considerando-se a competição pelos elétrons $4 \mathrm{~d} \pi$ do centro metálico, a frequência de estiramento $v_{\mathrm{CO}}$ diminui conforme a capacidade recebedora $\pi$ do ligante L diminui. Dessa forma, como o $v_{\mathrm{CO}}$ para o trans- $\left[\mathrm{Ru}(\mathrm{CO})\left(\mathrm{NH}_{3}\right)_{4}\left(\mathrm{P}\left(\mathrm{OCH}_{2} \mathrm{CH}_{3}\right)_{3}\right)\right]^{2+}$ ocorre em $1995 \mathrm{~cm}^{-1}$, pode-se considerar que o ligante de ácido fosforoso é um recebedor $\pi$ mais fraco $\left(v_{\mathrm{CO}}=1984\right.$ e $\left.1932 \mathrm{~cm}^{-1}\right)$ do que o trietil fosfito. De forma análoga, a forma protonada do ácido fosforoso $\left(v_{\mathrm{CO}}=1984 \mathrm{~cm}^{-1}\right)$ é um recebedor $\pi$ mais forte do que sua forma desprotonada $\left(v_{\mathrm{CO}}=1932 \mathrm{~cm}^{-1}\right)$. Isto está de acordo com a ordem de acidez $\pi$ determinada anteriormente por meio eletroquímico [46].

Em solução, o íon trans- $\left[\mathrm{Ru}(\mathrm{CO})\left(\mathrm{NH}_{3}\right)_{4}\left(\mathrm{P}(\mathrm{OH})_{3}\right)\right]^{2+}$ também exibe duas frequências de estiramento para o grupo CO (1984 e $\left.1968 \mathrm{~cm}^{-1}\right)$, Figura 29. O mesmo é observado por 
meio de cálculo DFT, sendo os valores teóricos de frequência de estiramento CO para as estruturas protonada e desprotonada de 1992 e $1961 \mathrm{~cm}^{-1}$, respectivamente, os quais são bastante próximos aos valores experimentais.

Figura 29 - Deconvolução das bandas de $\mathrm{CO}$ referente aos complexos trans- $\left[\mathrm{Ru}(\mathrm{CO})\left(\mathrm{NH}_{3}\right)_{4}\left(\mathrm{P}(\mathrm{OH})_{3}\right)\right]^{2+} /$ trans $-\left[\mathrm{Ru}(\mathrm{CO})\left(\mathrm{NH}_{3}\right)_{4}\left(\mathrm{P}(\mathrm{O})(\mathrm{OH})_{2}\right)\right]^{+}$em pH 3,0

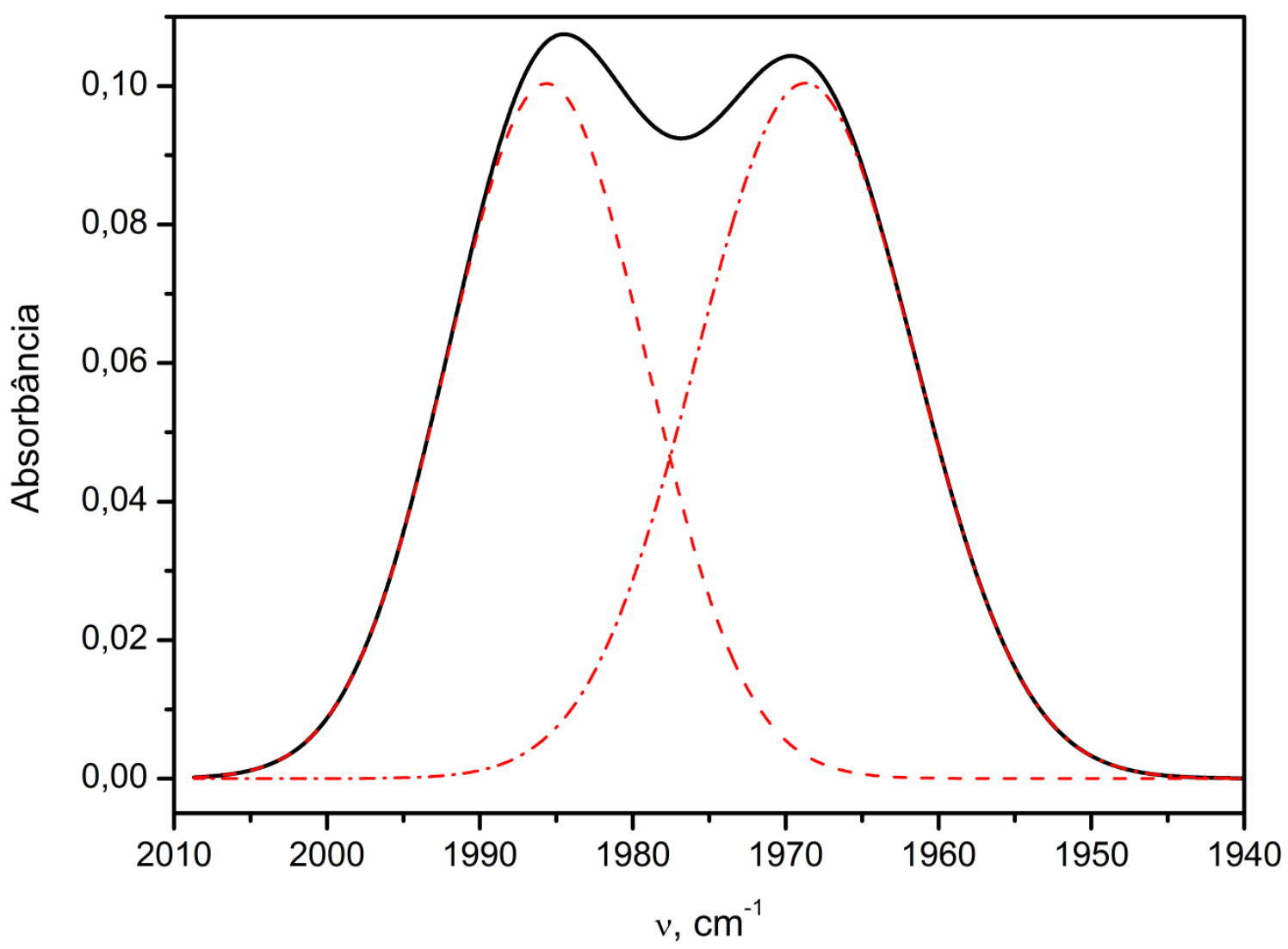

As áreas das bandas de $v_{\mathrm{CO}}$ para o complexo trans- $\left[\mathrm{Ru}(\mathrm{CO})\left(\mathrm{NH}_{3}\right)_{4}\left(\mathrm{P}(\mathrm{OH})_{3}\right)\right]^{2+}$ em solução aquosa são dependentes da concentração hidrogeniônica do meio, de forma que a área da banda em $1984 \mathrm{~cm}^{-1}$ diminui e a da banda em $1968 \mathrm{~cm}^{-1}$ aumenta conforme o valor do $\mathrm{pH}$ da solução aumentada.

A acidez de um ligante em um complexo de tetraaminas de rutênio é influenciada diretamente por dois fatores: o estado de oxidação do centro metálico e a natureza do ligante trans. Para exemplificar, no íon complexo trans-[Ru $\left.{ }^{\mathrm{II}}\left(\mathrm{H}_{2} \mathrm{O}\right)\left(\mathrm{NH}_{3}\right)_{5}\right]^{2+}$ a molécula de água 
apresenta $\mathrm{pK}_{\mathrm{a}}=13,1$, ao passo que no íon trans-[Ru $\left.{ }^{\mathrm{III}}\left(\mathrm{H}_{2} \mathrm{O}\right)\left(\mathrm{NH}_{3}\right)_{5}\right]^{3+}$ este valor é de 4,1 [95]. Ao alterar a natureza do ligante trans, substituindo-se o grupo $\mathrm{NH}_{3}$ por $\mathrm{NO}^{+}$, obtendo-se o íon complexo trans- $\left[\mathrm{Ru}(\mathrm{NO})\left(\mathrm{NH}_{3}\right)_{4}\left(\mathrm{H}_{2} \mathrm{O}\right)\right]^{3+}$, há um decréscimo no valor do $\mathrm{pK}_{\mathrm{a}}$ da água para 3,1 [95], o que demonstra que o ligante $\mathrm{NO}^{+}$torna o centro metálico um ácido de Lewis ainda mais forte que o $\mathrm{Ru}(\mathrm{III})$ no íon trans- $\left[\mathrm{Ru}^{\mathrm{III}}\left(\mathrm{H}_{2} \mathrm{O}\right)\left(\mathrm{NH}_{3}\right)_{5}\right]^{3+}$. Para o complexo trans- $\left[\mathrm{Ru}(\mathrm{CO})\left(\mathrm{NH}_{3}\right)_{4}\left(\mathrm{H}_{2} \mathrm{O}\right)\right]^{2+}$ o valor de $\mathrm{pK}_{\mathrm{a}}$ é de 7,7 [95], pois o CO é um recebedor $\pi$ mais fraco que o $\mathrm{NO}^{+}$. A acidez do ligante ácido fosforoso nos complexos do tipo trans- $\left[\mathrm{Ru}(\mathrm{L})\left(\mathrm{NH}_{3}\right)_{4}\left(\mathrm{P}(\mathrm{OH})_{3}\right)\right]^{\mathrm{n}}$ (reações 28-30) segue esta mesma tendência [56; 59].

$$
\begin{aligned}
& \text { trans }-\left[\mathrm{Ru}\left(\mathrm{H}_{2} \mathrm{O}\right)\left(\mathrm{NH}_{3}\right)_{4} \mathrm{P}(\mathrm{OH})_{3}\right]^{2+} \stackrel{\mathrm{H}_{2} \mathrm{O}}{\rightleftharpoons} \text { trans }-\left[\mathrm{Ru}\left(\mathrm{H}_{2} \mathrm{O}\right)\left(\mathrm{NH}_{3}\right)_{4} \mathrm{P}(\mathrm{O})(\mathrm{OH})_{2}\right]^{+} \quad \mathrm{pK}_{\mathrm{a}}=4,70 \\
& \text { trans }-\left[\mathrm{Ru}\left(\mathrm{H}_{2} \mathrm{O}\right)\left(\mathrm{NH}_{3}\right)_{4} \mathrm{P}(\mathrm{OH})_{3}\right]^{3+} \stackrel{\mathrm{H}_{2} \mathrm{O}}{\rightleftharpoons} \text { trans }-\left[\mathrm{Ru}\left(\mathrm{H}_{2} \mathrm{O}\right)\left(\mathrm{NH}_{3}\right)_{4} \mathrm{P}(\mathrm{O})(\mathrm{OH})_{2}\right]^{2+} \quad \mathrm{pK}_{\mathrm{a}}=1,00 \\
& \text { trans }-\left[\mathrm{Ru}(\mathrm{NO})\left(\mathrm{NH}_{3}\right)_{4} \mathrm{P}(\mathrm{OH})_{3}\right]^{3+} \stackrel{\mathrm{H}_{2} \mathrm{O}}{\rightleftharpoons} \text { trans }-\left[\mathrm{Ru}(\mathrm{NO})\left(\mathrm{NH}_{3}\right)_{4} \mathrm{P}(\mathrm{O})(\mathrm{OH})_{2}\right]^{2+} \quad \mathrm{pK}_{\mathrm{a}}=0,74
\end{aligned}
$$

Dessa forma, espera-se que o $\mathrm{pK}_{\mathrm{a}}$ do ácido fosforoso no complexo trans- $\left[\mathrm{Ru}(\mathrm{CO})\left(\mathrm{NH}_{3}\right)_{4}\left(\mathrm{P}(\mathrm{OH})_{3}\right)\right]^{2+}$ tenha valor superior a 0,74 e inferior a 4,7 (reações 30 e 28). A Figura 30 mostra a variação da área da banda em $1968 \mathrm{~cm}^{-1}$ em função do pH. A derivada primeira deste gráfico permite calcular o $\mathrm{pK}_{\mathrm{a}}$ para a reação 31 como sendo 3,3, conforme esperado.

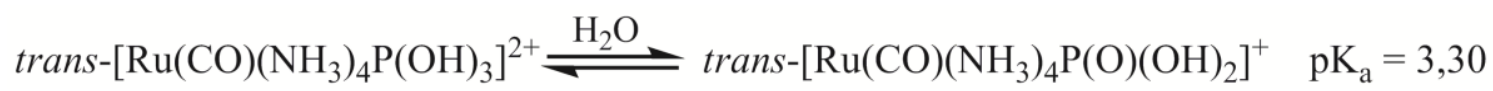


Figura 30 - Sigmoidal obtida por meio da área da banda de $\mathrm{CO}$ em $1868 \mathrm{~cm}^{-1}$, referente ao íon trans- $\left[\mathrm{Ru}(\mathrm{CO})\left(\mathrm{NH}_{3}\right)_{4}\left(\mathrm{P}(\mathrm{O})(\mathrm{OH})_{2}\right)\right]^{+}$, em diferentes concentrações hidrogeniônicas, $\mathrm{pK}_{\mathrm{a}}=3,30$

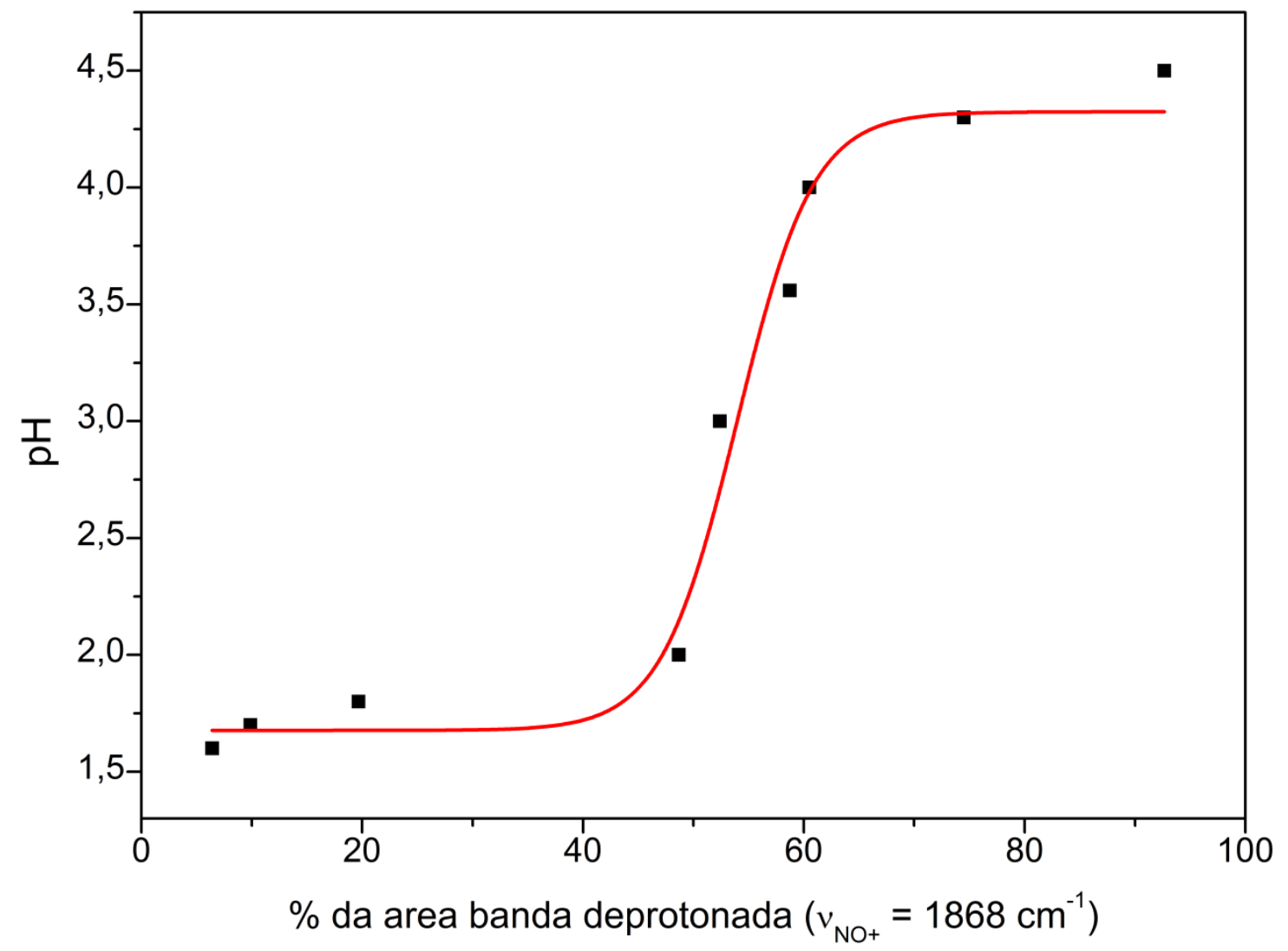

\subsubsection{Ressonância Magnética Nuclear $\mathrm{de}^{31} \mathrm{P}$}

O espectro de ${ }^{31} \mathrm{P}$ RMN do carbonilo complexo de ácido fosforoso em solução pH 7,5 apresentou apenas um deslocamento químico em 106 ppm (Figura 31 a). Ao acidificar esta solução até pH 1,0 este sinal desaparece havendo a formação de um sinal em 118 ppm (Figura 31 b). Considerando que o $\mathrm{pK}_{\mathrm{a}}$ deste complexo é 3,30, atribuiu-se o sinal em 118 ppm ao íon trans- $\left[\mathrm{Ru}(\mathrm{CO})\left(\mathrm{NH}_{3}\right)_{4}\left(\mathrm{P}(\mathrm{OH})_{3}\right)\right]^{2+}$, em que o átomo de fósforo encontra-se menos blindado, e o sinal em 106 ppm ao íon trans- $\left[\mathrm{Ru}(\mathrm{CO})\left(\mathrm{NH}_{3}\right)_{4}\left(\mathrm{P}(\mathrm{O})(\mathrm{OH})_{2}\right)\right]^{+}$em que o átomo de fósforo está mais blindado devido a deprotonação do oxigênio. Os cálculos teóricos realizados para estas estruturas estão de acordo tais atribuições de deslocamento químico de ${ }^{31} \mathrm{P}$, uma vez que 
as cargas de Mulliken para o átomo de fósforo são 1,207 e 1,223 para as espécies protonada e desprotonada, respectivamente.

Figura $31-{ }^{31} \mathrm{P}$ RMN do complexo trans- $\left[\mathrm{Ru}(\mathrm{CO})\left(\mathrm{NH}_{3}\right)_{4}\left(\mathrm{P}(\mathrm{O})(\mathrm{OH})_{2}\right)\right](\mathrm{Cl}), \mathrm{C}_{\mathrm{Ru}}=2,0 \times 10^{-2}$ mol L ${ }^{-1}, \mathrm{NH}_{4} \mathrm{PF}_{6}$ como padrão interno $\left(\delta=-144 \mathrm{ppm}\right.$ ), temperatura de $25 \pm 0,5^{\circ} \mathrm{C}$, (a) solução pH 7,5 (TRIS), (b) Após adição de $\mathrm{CF}_{3} \mathrm{COOH} \mathrm{6,0} \mathrm{mol} \mathrm{L}^{-1}, \mathrm{pH} 1,0$.

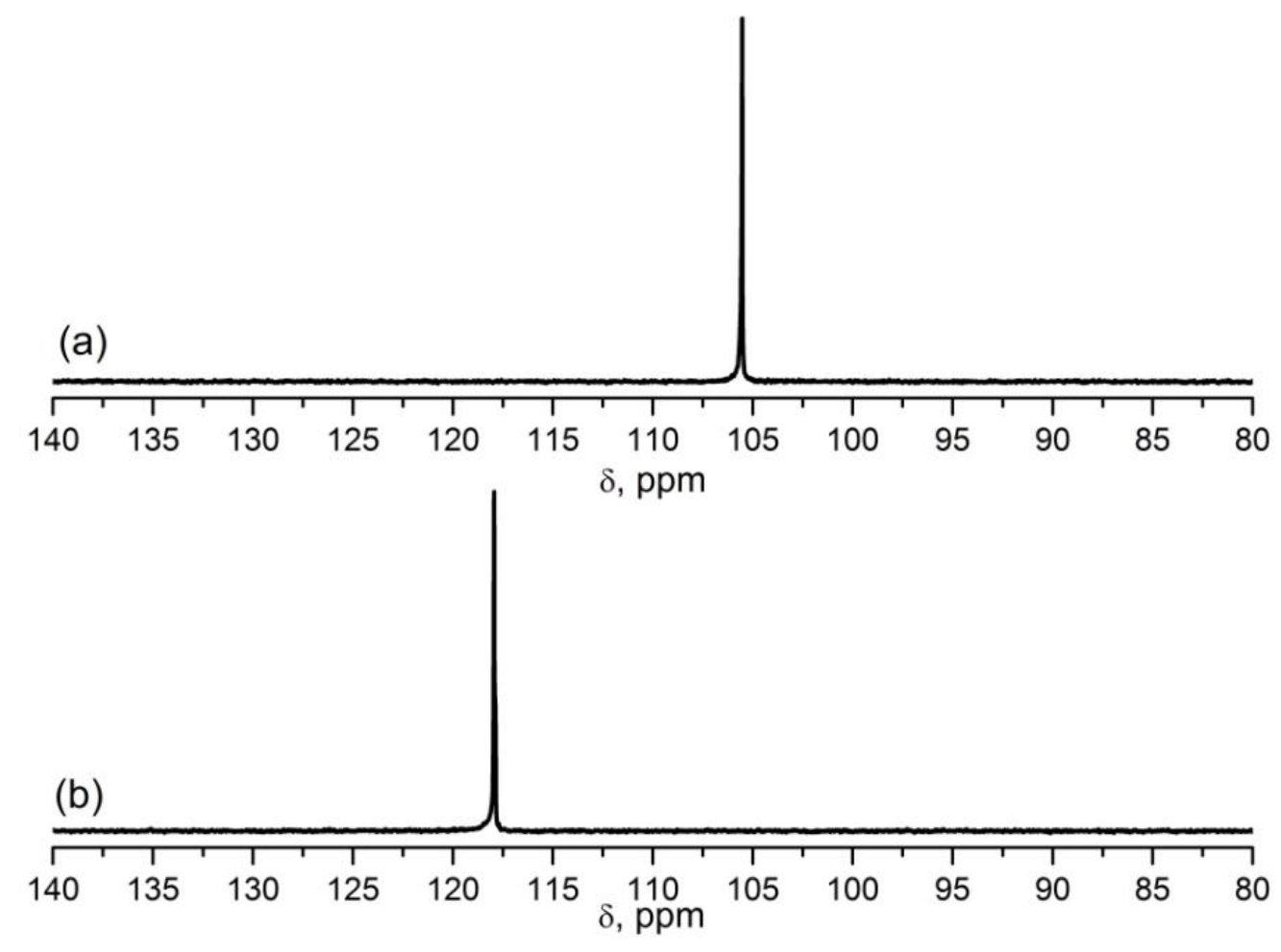

\subsubsection{Estudo da estabilidade do complexo trans- $\left[\mathrm{Ru}(\mathrm{CO})\left(\mathrm{NH}_{3}\right)_{4}\left(\mathrm{P}(\mathrm{OH})_{3}\right)\right](\mathrm{Cl})_{3} \mathrm{em}$}

\section{solução}

O íon trans- $\left[\mathrm{Ru}(\mathrm{CO})\left(\mathrm{NH}_{3}\right)_{4}\left(\mathrm{P}(\mathrm{OH})_{3}\right)\right]^{2+}$ foi monitorado em meio ácido $(\mathrm{pH} 3,0, \mu=0,1$ mol $\mathrm{L}^{-1}, 25 \pm 0,1^{\circ} \mathrm{C}$ ) por espectroscopia eletrônica e de infravermelho durante 48 horas e não apresentou modificações espectrais.

Contudo, íon trans- $\left[\mathrm{Ru}(\mathrm{CO})\left(\mathrm{NH}_{3}\right)_{4}\left(\mathrm{P}(\mathrm{O})(\mathrm{OH})_{2}\right)\right]^{+}$em solução $\mathrm{pH} 7,5$ foi monitorado por 72 horas por UV-vis e apresentou, em função do tempo, um decréscimo na intensidade da 
banda na região de $220 \mathrm{~nm}$ e um aumento na intensidade das bandas entre 270 e $400 \mathrm{~nm}$ (Figura 32). A constante de velocidade observada (k) para a formação da banda em $287 \mathrm{~nm}$ foi estimada como $1,10 \times 10^{-5} \mathrm{~s}^{-1}\left(\mathrm{t}_{1 / 2}=17,5 \mathrm{~h}\right)$.

Figura 32 - Espectros eletrônico do íon trans- $\left[\mathrm{Ru}(\mathrm{CO})\left(\mathrm{NH}_{3}\right)_{4}\left(\mathrm{P}(\mathrm{O})(\mathrm{OH})_{2}\right)\right]^{+}$em função do tempo em solução pH 7,5 (Tampão TRIS), $\mu=0,1 \mathrm{~mol} \mathrm{~L}^{-1} \mathrm{CF}_{3} \mathrm{COONa}, 25 \pm 0,1^{\circ} \mathrm{C}$, cubeta de $1,0 \mathrm{~cm}$ de caminho ótico e $\mathrm{C}_{\mathrm{Ru}}=4,0 \times 10^{-4} \mathrm{~mol} \mathrm{~L}^{-1}$

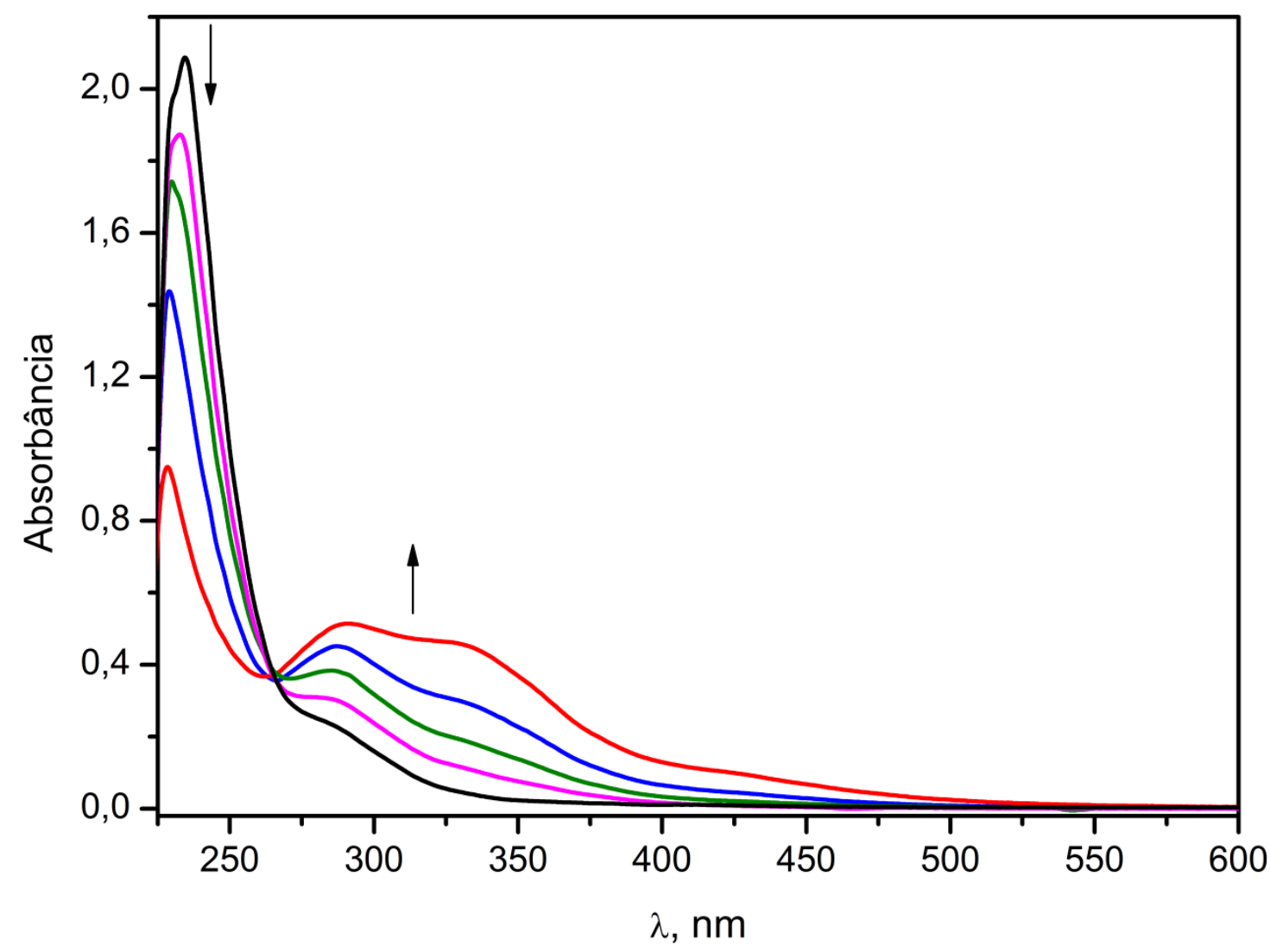

Soluções contendo o íon trans- $\left[\mathrm{Ru}(\mathrm{CO})\left(\mathrm{NH}_{3}\right)_{4}\left(\mathrm{P}(\mathrm{O})(\mathrm{OH})_{2}\right)\right]^{+}$também foram monitoradas por espectroscopia de infravermelho em pH 7,5 durante 72 horas. Neste caso, observou-se apenas um leve aumento da intensidade da banda em $1968 \mathrm{~cm}^{-1}$, não sendo observado o aparecimento de nenhuma nova $v_{\mathrm{CO}}$ (Figura 33).

Espectros de ${ }^{31} \mathrm{P}$ RMN do carbonilo trans- $\left[\mathrm{Ru}(\mathrm{CO})\left(\mathrm{NH}_{3}\right)_{4}\left(\mathrm{P}(\mathrm{O})(\mathrm{OH})_{2}\right)\right]^{+}$em solução pH 7,5 indicaram que após 72 horas, apenas uma baixa porcentagem (>5\%) do ácido 
fosforoso é descoordenado, sendo que o deslocamento químico do átomo de fósforo coordenado permaneceu em 106 ppm e não ocorreu a formação de nenhum outro sinal em região de fósforo coordenado. O acompanhamento desta solução por ${ }^{31} \mathrm{P}$ RMN pelo período de 65 dias mostra que o ácido fosforoso é lentamente dissociado com uma constante observada de $\mathrm{k}=1,75 \times 10^{-7} \mathrm{~s}^{-1}$.

Figura 33 - Espectro na região do infravermelho do íon trans- $\left[\mathrm{Ru}(\mathrm{CO})\left(\mathrm{NH}_{3}\right)_{4}\left(\mathrm{P}(\mathrm{O})(\mathrm{OH})_{2}\right)\right]^{+}$ em solução pH 7,5 após 1 minuto e após 72 horas, obtido em pastilhas de silício, com espaçador de teflon de $0,10 \mathrm{~mm} \mathrm{e} \mathrm{C}_{\mathrm{Ru}}=5,0 \times 10^{-2} \mathrm{~mol} \mathrm{~L}^{-1}$.

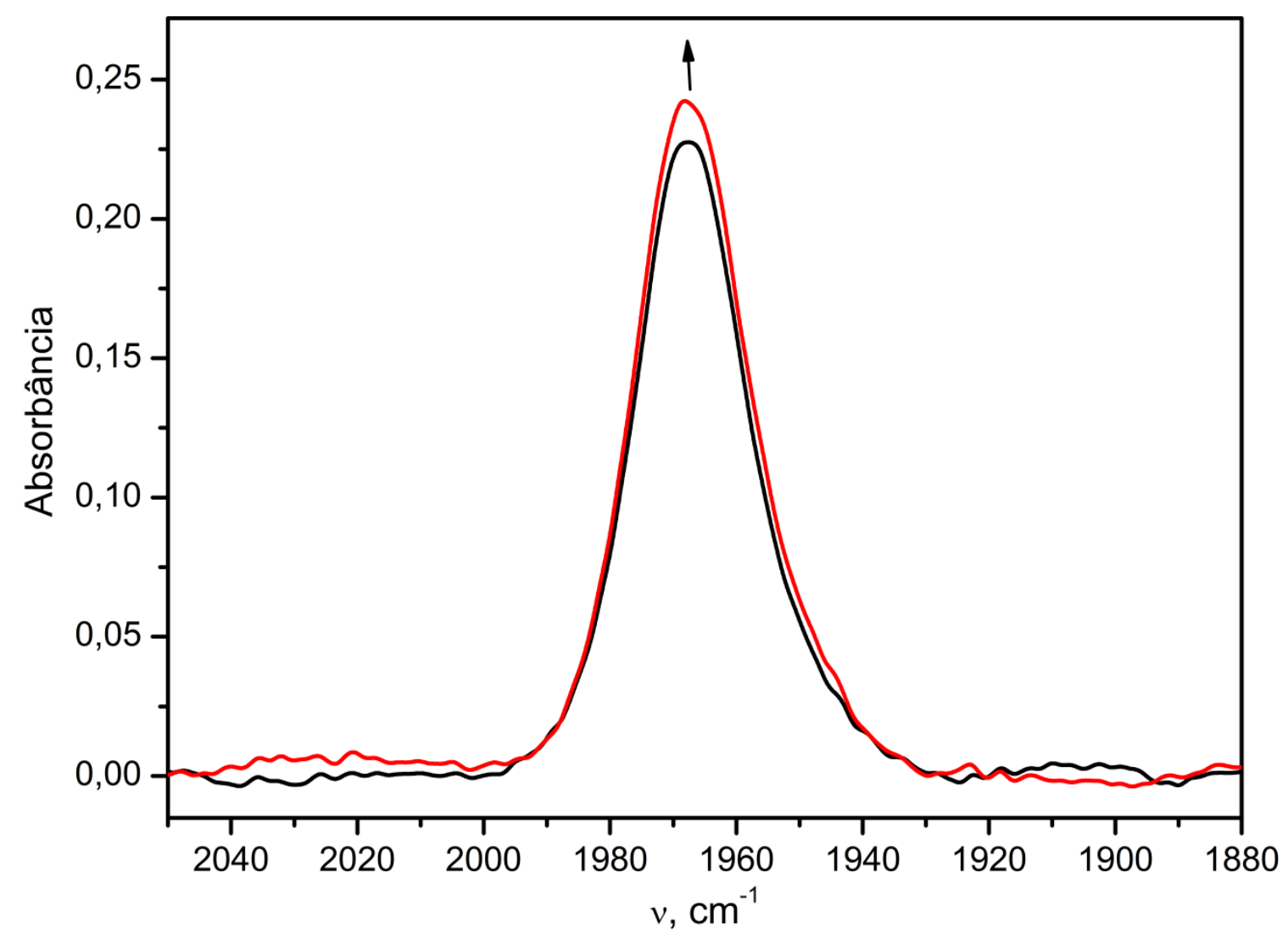

A princípio pode-se admitir que o comportamento do íon trans- $\left[\mathrm{Ru}(\mathrm{CO})\left(\mathrm{NH}_{3}\right)_{4}\left(\mathrm{P}(\mathrm{O})(\mathrm{OH})_{2}\right)\right]^{+}$em solução poderia ser explicado tentativamente com base na aquação do grupo $\mathrm{CO}$ e/ou na aquação do ácido fosforoso desprotonado (reações 32 e 33). 


$$
\begin{aligned}
& \text { trans }-\left[\mathrm{Ru}(\mathrm{CO})\left(\mathrm{NH}_{3}\right)_{4} \mathrm{P}(\mathrm{O})(\mathrm{OH})_{2}\right]^{+} \stackrel{\mathrm{H}_{2} \mathrm{O}}{\longrightarrow} \text { trans }-\left[\mathrm{Ru}\left(\mathrm{H}_{2} \mathrm{O}\right)\left(\mathrm{NH}_{3}\right)_{4} \mathrm{P}(\mathrm{O})(\mathrm{OH})_{2}\right]^{+}+\mathrm{CO} \\
& \text { trans }-\left[\mathrm{Ru}(\mathrm{CO})\left(\mathrm{NH}_{3}\right)_{4} \mathrm{P}(\mathrm{O})(\mathrm{OH})_{2}\right]^{+} \stackrel{\mathrm{H}_{2} \mathrm{O}}{\longrightarrow} \text { trans }-\left[\mathrm{Ru}(\mathrm{CO})\left(\mathrm{NH}_{3}\right)_{4}\left(\mathrm{H}_{2} \mathrm{O}\right)\right]^{2+}+\mathrm{P}(\mathrm{O})(\mathrm{OH})_{2}
\end{aligned}
$$

A deconvolução do último espectro da Figura 32 permitiu observar três bandas em 335 $\mathrm{nm}\left(\varepsilon=1052 \mathrm{~L} \mathrm{~mol}^{-1} \mathrm{~cm}^{-1}\right), 287 \mathrm{~nm}\left(\varepsilon=1185 \quad \mathrm{~L}^{-1} \mathrm{~cm}^{-1}\right)$ e $232 \mathrm{~nm}$ $\left(\varepsilon=2150 \mathrm{~L} \mathrm{~mol}^{-1} \mathrm{~cm}^{-1}\right)$. Entretanto, o íon trans- $\left[\mathrm{Ru}\left(\mathrm{H}_{2} \mathrm{O}\right)\left(\mathrm{NH}_{3}\right)_{4}\left(\mathrm{P}(\mathrm{O})(\mathrm{OH})_{2}\right)\right]^{+}$, produto da reação 32, apresenta uma banda característica em $316 \mathrm{~nm}\left(\varepsilon=530 \mathrm{~L} \mathrm{~mol}^{-1} \mathrm{~cm}^{-1}\right)$ [56], e o trans- $\left[\mathrm{Ru}(\mathrm{CO})\left(\mathrm{NH}_{3}\right)_{4}\left(\mathrm{H}_{2} \mathrm{O}\right)\right]^{2+}$ produto da reação 33, apresenta uma banda característica em $281 \mathrm{~nm}\left(\varepsilon=440 \mathrm{~L} \mathrm{~mol}^{-1} \mathrm{~cm}^{-1}\right)$ [107]. Estes dois possíveis produtos apresentam bandas com absortividade molar inferior às observadas na Figura 32. Em adição, o experimento de ${ }^{31} \mathrm{P}$ RMN em pH 7,5 em função do tempo mostra que não houve formação da espécie trans- $\left[\mathrm{Ru}\left(\mathrm{H}_{2} \mathrm{O}\right)\left(\mathrm{NH}_{3}\right)_{4}\left(\mathrm{P}(\mathrm{O})(\mathrm{OH})_{2}\right)\right]^{+}$, além da frequência de estiramento para o trans- $\left[\mathrm{Ru}(\mathrm{CO})\left(\mathrm{NH}_{3}\right)_{4}\left(\mathrm{H}_{2} \mathrm{O}\right)\right]^{2+}\left(v_{\mathrm{CO}}=1919 \mathrm{~cm}^{-1}\right)$ [107] não ter sido observada.

O aumento da intensidade das bandas na região entre 280 e $340 \mathrm{~nm}$ (Figura 32)e o aumento da intensidade do $v_{\mathrm{CO}}$ (Figura 33) compõem um comportamento químico semelhante ao observado para o íon trans- $\left[\mathrm{Ru}(\mathrm{NO})\left(\mathrm{NH}_{3}\right)_{4}\left(\mathrm{P}(\mathrm{O})(\mathrm{OH})_{2}\right)\right]^{2+}$, no qual o ligante $\mathrm{P}(\mathrm{O})(\mathrm{OH})_{2}$ sofre isomerização formando os complexos trans- $\left[\mathrm{Ru}(\mathrm{NO})\left(\mathrm{NH}_{3}\right)_{4}\left(\mathrm{OP}(\mathrm{OH})_{2}\right)\right]^{2+}$ e trans- $\left[\mathrm{Ru}(\mathrm{NO})\left(\mathrm{NH}_{3}\right)_{4}\left(\mathrm{OP}(\mathrm{H})(\mathrm{OH})_{2}\right)\right]^{3+}$ (seção 4.1) [59]. Todavia, no caso do nitrosilo complexo tanto o deslocamento químico do átomo de fósforo quanto a frequência de estiramento do grupo $\mathrm{NO}^{+}$sofrem alteração, o que não é observado no caso do carbonilo complexo de ácido fosforoso.

Considerando-se a possibilidade de formação das mesmas estruturas intermediárias observadas para o nitrosilo complexo de ácido fosforoso, foram realizados cálculos DFT para estas estruturas do carbonilo complexo de ácido fosforoso. A Figura 34 apresenta tais 
estruturas bem como os valores teóricos calculados de frequência de estiramento $\mathrm{CO}$ e de carga sobre o átomo de fósforo.

Figura 34 - Valores teóricos calculados para a frequência de estiramento $\mathrm{CO}$ e carga de Mulliken para o átomo de fósforo para as possíveis estruturas do carbonilo complexo de ácido fosforoso

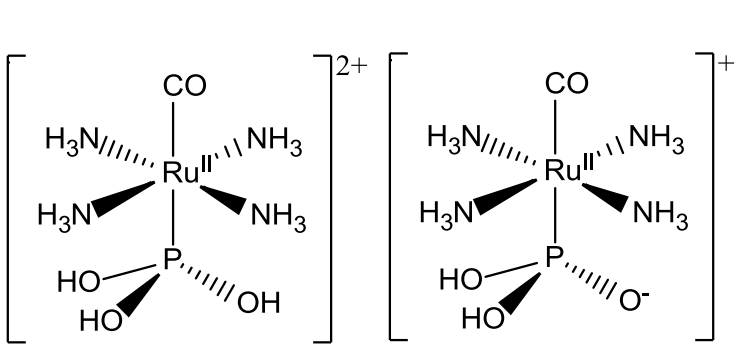

Carga $\mathrm{P}=1,257$

$v_{\mathrm{CO}}=1992 \mathrm{~cm}^{-1}$

$\mathrm{w}_{1 / 2}=15 \mathrm{~cm}^{-1}$
Carga $\mathrm{P}=1,223$

$v_{\mathrm{CO}}=1961 \mathrm{~cm}^{-1}$

$\mathrm{w}_{1 / 2}=15 \mathrm{~cm}^{-1}$


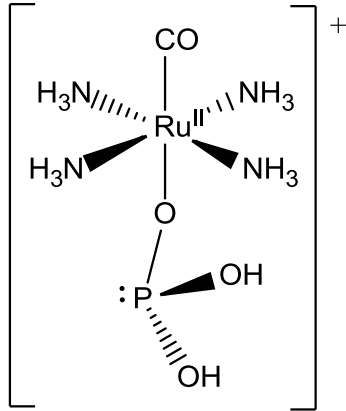

Carga $\mathrm{P}=0,752$

$v_{\mathrm{CO}}=1923 \mathrm{~cm}^{-1}$

$\mathrm{w}_{1 / 2}=17 \mathrm{~cm}^{-1}$

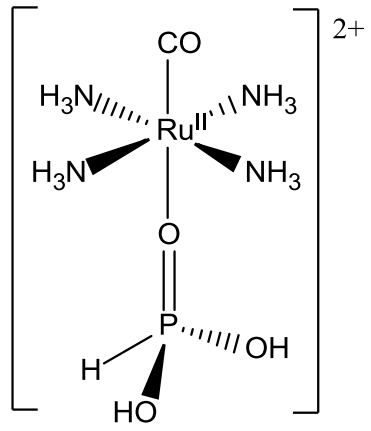

Carga $\mathrm{P}=1,212$

$$
\begin{aligned}
& v_{\mathrm{CO}}=1951 \mathrm{~cm}^{-1} \\
& \mathrm{w}_{1 / 2}=15,6 \mathrm{~cm}^{-1}
\end{aligned}
$$

A frequência de estiramento do grupo $\mathrm{CO}$ e a carga sobre o átomo de fósforo (Figura 34) seguem a mesma ordem observada experimentalmente para as estruturas trans $-\left[\mathrm{Ru}(\mathrm{CO})\left(\mathrm{NH}_{3}\right)_{4}\left(\mathrm{P}(\mathrm{OH})_{3}\right)\right]^{2+}\left(v_{\mathrm{CO}}=1984 \mathrm{~cm}^{-1}, \quad \delta_{31 \mathrm{P}}=118 \mathrm{ppm}\right) \mathrm{e}$ trans $-\left[\mathrm{Ru}(\mathrm{CO})\left(\mathrm{NH}_{3}\right)_{4}\left(\mathrm{P}(\mathrm{O})(\mathrm{OH})_{2}\right)\right]^{+}\left(v_{\mathrm{CO}}=1968 \mathrm{~cm}^{-1}, \delta_{31 \mathrm{P}}=106 \mathrm{ppm}\right)$. Entretanto, os resultados calculados para a estrutura em que o ácido fosforoso, ainda em sua forma piramidal, está coordenado ao centro metálico pelo átomo de oxigênio (trans- $\left.\left[\mathrm{Ru}(\mathrm{CO})\left(\mathrm{NH}_{3}\right)_{4}\left((\mathrm{O}) \mathrm{P}(\mathrm{OH})_{2}\right)\right]^{+}\right)$apresenta $v_{\mathrm{CO}}$ e carga sobre o átomo de fósforo consideravelmente diferentes das demais estruturas. Isto não foi observado experimentalmente nem por espectroscopia vibracional nem por ${ }^{31} \mathrm{P}$ RMN. Já a estrutura trans- $\left[\mathrm{Ru}(\mathrm{CO})\left(\mathrm{NH}_{3}\right)_{4}\left((\mathrm{O}) \mathrm{P}(\mathrm{H})(\mathrm{OH})_{2}\right)\right]^{2+}$ apresenta valores $v_{\mathrm{CO}}$ e carga sobre o átomo de fósforo próximos aos calculados para a estrutura trans- $\left[\mathrm{Ru}(\mathrm{CO})\left(\mathrm{NH}_{3}\right)_{4}\left(\mathrm{P}(\mathrm{O})(\mathrm{OH})_{2}\right)\right]^{+}$, 
havendo a possibilidade de que suas frequências de estiramento $\mathrm{CO}$ e deslocamentos químicos estejam sobrepostos.

Figura 35 - Espectro de infravermelho teórico para os carbonilos complexos de ácido fosforoso: trans- $\left[\mathrm{Ru}(\mathrm{CO})\left(\mathrm{NH}_{3}\right)_{4}\left(\mathrm{P}(\mathrm{O})(\mathrm{OH})_{3}\right)\right]^{+}$e trans $-\left[\mathrm{Ru}(\mathrm{CO})\left(\mathrm{NH}_{3}\right)_{4}\left((\mathrm{O}) \mathrm{P}(\mathrm{H})(\mathrm{OH})_{3}\right)\right]^{2+}$

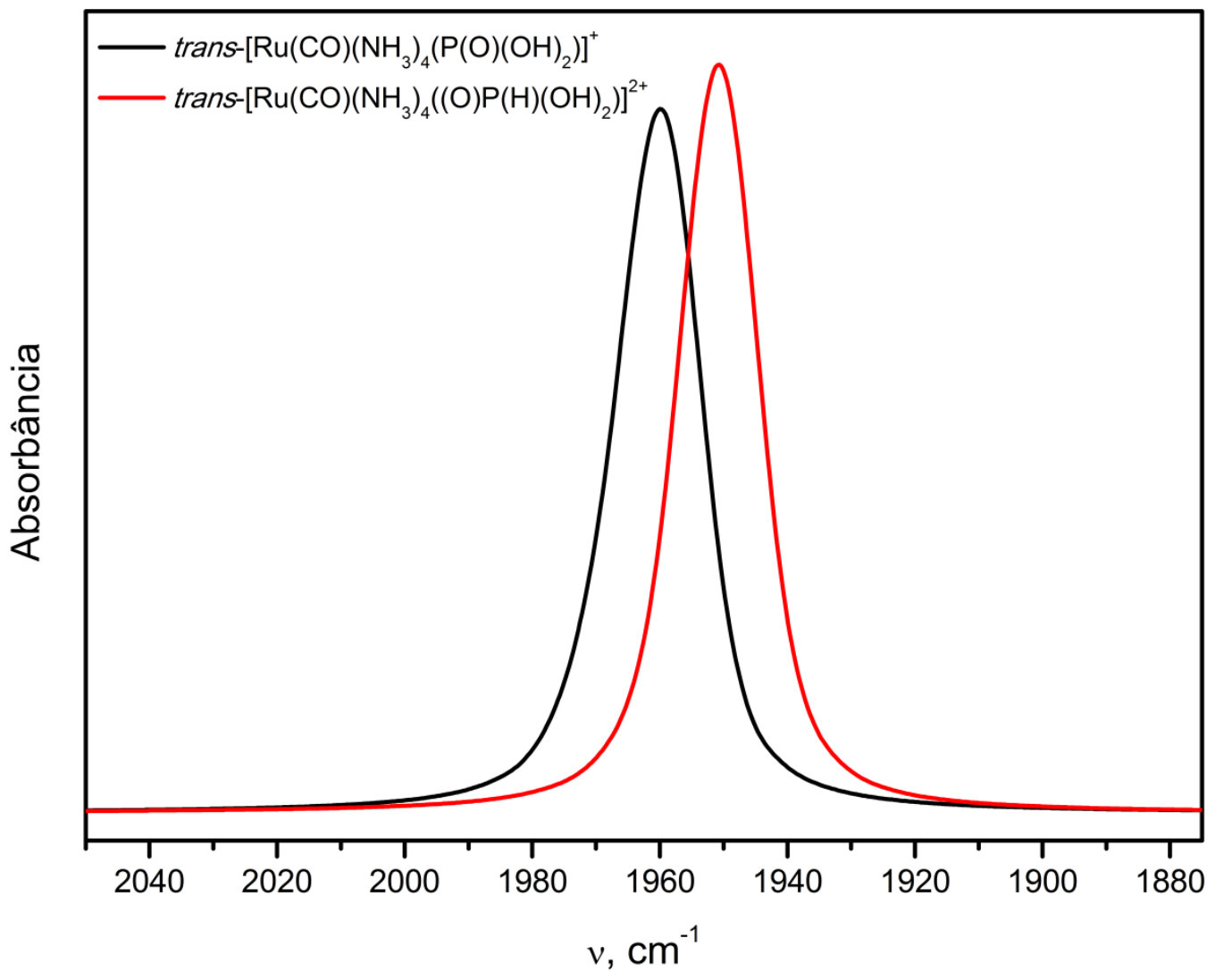

Os espectros de infravermelho experimentais obtidos para as espécies trans $-\left[\mathrm{Ru}(\mathrm{CO})\left(\mathrm{NH}_{3}\right)_{4}\left(\mathrm{P}(\mathrm{OH})_{3}\right)\right]^{2+}$ e trans- $\left[\mathrm{Ru}(\mathrm{CO})\left(\mathrm{NH}_{3}\right)_{4}\left(\mathrm{P}(\mathrm{O})(\mathrm{OH})_{2}\right)\right]^{+}$apresentaram banda com largura a meia altura $\left(\mathrm{w}_{1 / 2}\right)$ de $17 \mathrm{~cm}^{-1}$. Entretanto, o espectro de infravermelho obtido em pH 7,5 apresenta $\mathrm{w}_{1 / 2}=21 \mathrm{~cm}^{-1}$. Os espectros de infravermelho teórico para as mesmas espécies trans- $\left[\mathrm{Ru}(\mathrm{CO})\left(\mathrm{NH}_{3}\right)_{4}\left(\mathrm{P}(\mathrm{OH})_{3}\right)\right]^{2+}$ e trans- $\left[\mathrm{Ru}(\mathrm{CO})\left(\mathrm{NH}_{3}\right)_{4}\left(\mathrm{P}(\mathrm{O})(\mathrm{OH})_{2}\right)\right]^{+}$apresentam $\mathrm{w}_{1 / 2}=15 \mathrm{~cm}^{-1}$ (Figura 35), o que demonstra que o calculo teórico descreve de forma eficiente o sistema em estudo. Quando a $v_{\mathrm{CO}}$ teórica para as espécies trans- $\left[\mathrm{Ru}(\mathrm{CO})\left(\mathrm{NH}_{3}\right)_{4}\left(\mathrm{P}(\mathrm{O})(\mathrm{OH})_{2}\right)\right]^{+}$e trans- $\left[\mathrm{Ru}(\mathrm{CO})\left(\mathrm{NH}_{3}\right)_{4}\left((\mathrm{O}) \mathrm{P}(\mathrm{H})(\mathrm{OH})_{2}\right)\right]^{2+}$ são colocadas 
no mesmo gráfico (Figura 35) é possível observar uma considerável região de sobreposição e a $\mathrm{w}_{1 / 2}$ para ambas as $v_{\mathrm{CO}}$ sobrepostas passa a ser de $25 \mathrm{~cm}^{-1}$, o que poderia explicar o valor de $\mathrm{w}_{1 / 2}=21 \mathrm{~cm}^{-1}$ para $v_{\mathrm{CO}}$ obtida experimentalmente em pH 7,5. Em adição, observa-se que a absortividade molar da $v_{\mathrm{CO}}$ para a espécie trans- $\left[\mathrm{Ru}(\mathrm{CO})\left(\mathrm{NH}_{3}\right)_{4}\left((\mathrm{O}) \mathrm{P}(\mathrm{H})(\mathrm{OH})_{2}\right)\right]^{2+}$ é ligeiramente maior do que a calculada para a espécie trans- $\left[\mathrm{Ru}(\mathrm{CO})\left(\mathrm{NH}_{3}\right)_{4}\left(\mathrm{P}(\mathrm{O})(\mathrm{OH})_{2}\right)\right]^{+}$, o que também está de acordo com o observado experimentalmente (Figura 33).

Figura 36 - Espectro eletrônico teórico (TD-DFT) para os carbonilos complexos de ácido fosforoso: trans- $\left[\mathrm{Ru}(\mathrm{CO})\left(\mathrm{NH}_{3}\right)_{4}\left(\mathrm{P}(\mathrm{O})(\mathrm{OH})_{3}\right)\right]^{+}, \quad$ trans $-\left[\mathrm{Ru}(\mathrm{CO})\left(\mathrm{NH}_{3}\right)_{4}\left((\mathrm{O}) \mathrm{P}(\mathrm{OH})_{2}\right)\right]^{+} \quad \mathrm{e}$ trans- $\left[\mathrm{Ru}(\mathrm{CO})\left(\mathrm{NH}_{3}\right)_{4}\left((\mathrm{O}) \mathrm{P}(\mathrm{H})(\mathrm{OH})_{3}\right)\right]^{2+}$

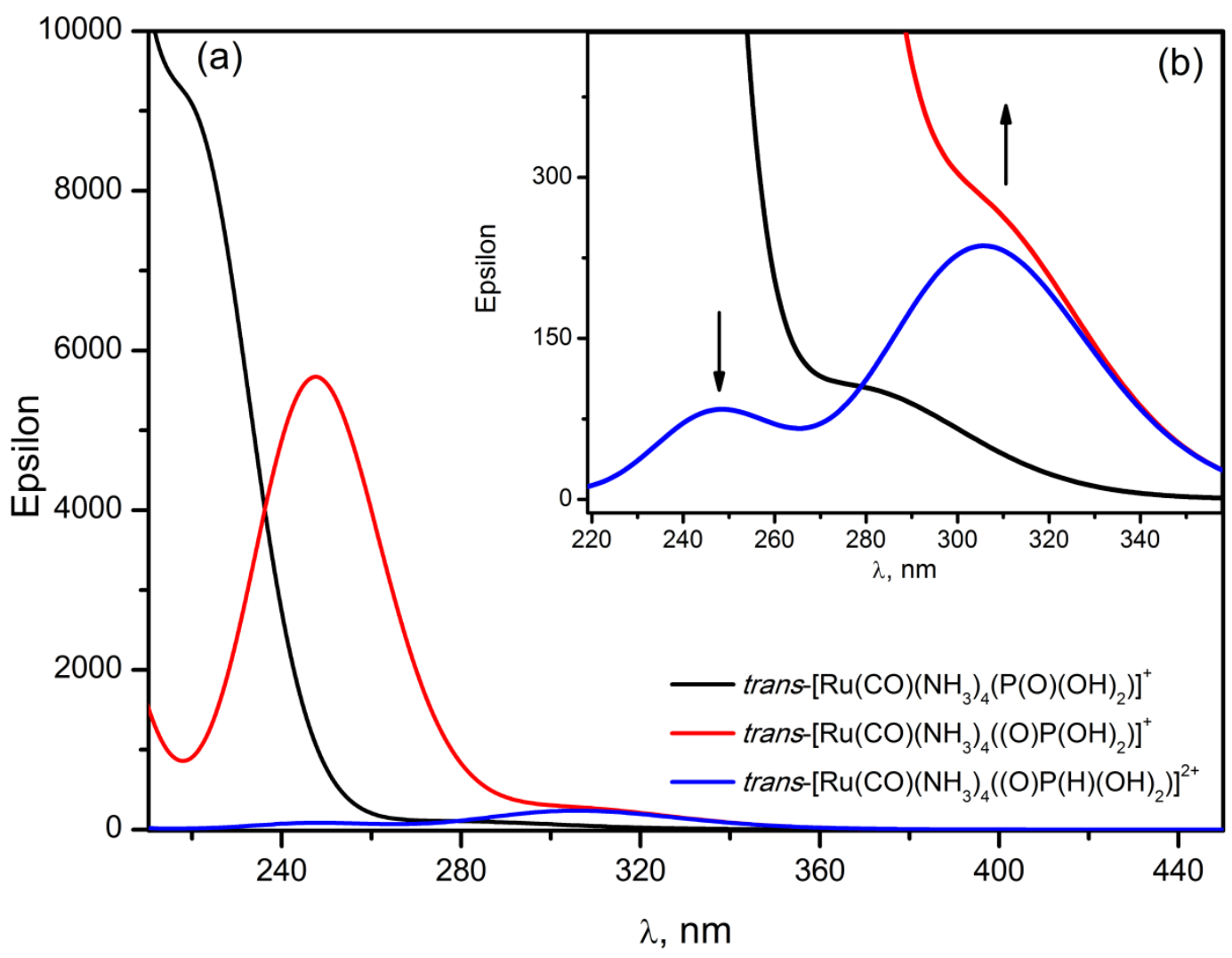

Os espectros eletrônicos dos carbonilos complexos de ácido fosforoso: trans- $\left[\mathrm{Ru}(\mathrm{CO})\left(\mathrm{NH}_{3}\right)_{4}\left(\mathrm{P}(\mathrm{O})(\mathrm{OH})_{3}\right)\right]^{+}, \quad$ trans $-\left[\mathrm{Ru}(\mathrm{CO})\left(\mathrm{NH}_{3}\right)_{4}\left((\mathrm{O}) \mathrm{P}(\mathrm{OH})_{2}\right)\right]^{+} \quad \mathrm{e}$ trans- $\left[\mathrm{Ru}(\mathrm{CO})\left(\mathrm{NH}_{3}\right)_{4}\left((\mathrm{O}) \mathrm{P}(\mathrm{H})(\mathrm{OH})_{3}\right)\right]^{2+}$ foram calculados utilizando TD-DFT (Figura 36). Os 
complexos em que o ácido fosforoso está coordenado pelo átomo de oxigênio apresentam bandas na região de 280 e $340 \mathrm{~nm}$ com maior absortividade molar (Figura 36 b) e bandas na região de maior energia $(\sim 250 \mathrm{~nm})$ com menor absortividade molar (Figura 36 a) do que o complexo de ácido fosforoso coordenado pelo átomo de fósforo. Isto é semelhante ao observado experimentalmente em pH 7,5 (Figura 32).

Figura 37 - Dissociação do ligante de ácido fosforoso a partir do complexo trans- $\left[\mathrm{Ru}(\mathrm{CO})\left(\mathrm{NH}_{3}\right)_{4}\left(\mathrm{P}(\mathrm{OH})_{3}\right)\right]^{2+}$

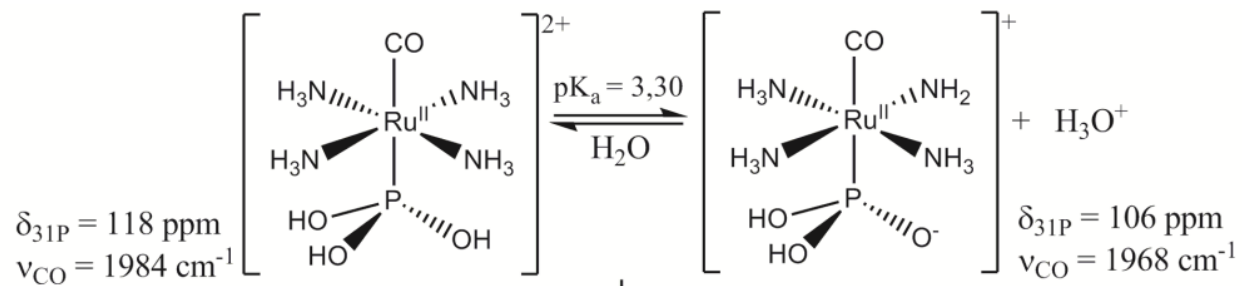

$$
\begin{aligned}
& \begin{array}{r|}
\mathrm{pH} 7,50 \\
25^{\circ} \mathrm{C}
\end{array} \mathrm{k}=1,10 \times 10^{-5} \mathrm{~s}^{-1} \\
& {\left[{ }_{3}\right.} \\
& \underset{25^{\circ} \mathrm{C}}{\mathrm{pH} 7,50} \mathrm{pK}_{\mathrm{a}}>7,5 \\
& {\left[\begin{array}{l}
\delta_{31 \mathrm{P}}=106 \mathrm{ppm}^{2+} \\
v_{\mathrm{CO}}=1968 \mathrm{~cm}^{-1}
\end{array}\right.} \\
& \left.\underset{25^{\circ} \mathrm{C}}{\mathrm{pH} 7,50}\right\rfloor \mathrm{k}=1,75 \times 10^{-7} \mathrm{~s}^{-1}
\end{aligned}
$$

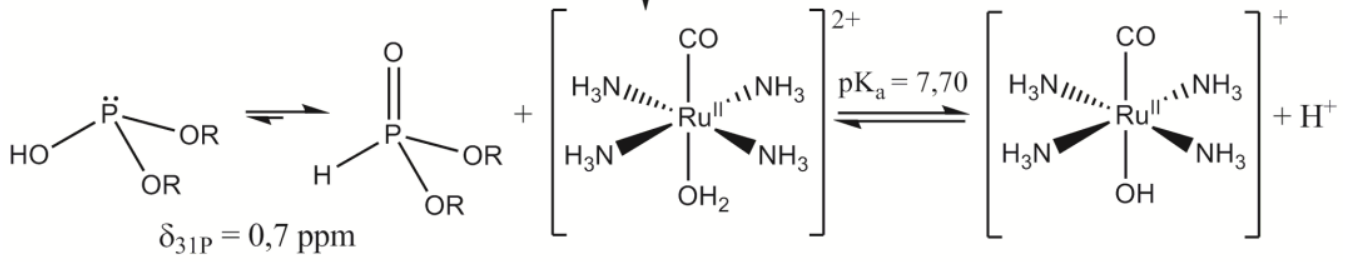


Assim, os dados experimentais e teóricos de espectroscopia eletrônica e vibracional indicam que o ácido fosforoso no íon trans- $\left[\mathrm{Ru}(\mathrm{CO})\left(\mathrm{NH}_{3}\right)_{4}\left(\mathrm{P}(\mathrm{O})(\mathrm{OH})_{2}\right)\right]^{+}$muda sua coordenação do átomo de fósforo para o átomo de oxigênio, o que, de acordo com a variação no espectro eletrônico (Figura 32), ocorreria com $\mathrm{k}=1,10 \times 10^{-5} \mathrm{~s}^{-1}$.

Todavia a estrutura intermediária trans-[Ru(CO) $\left.\left(\mathrm{NH}_{3}\right)_{4}\left((\mathrm{O}) \mathrm{P}(\mathrm{OH})_{2}\right)\right]^{+}$não foi observada experimentalmente por espectroscopia de infravermelho e ${ }^{31} \mathrm{P}$ RMN. Isto talvez deva-se ao fato da espécie trans- $\left[\mathrm{Ru}(\mathrm{CO})\left(\mathrm{NH}_{3}\right)_{4}\left((\mathrm{O}) \mathrm{P}(\mathrm{H})(\mathrm{OH})_{2}\right)\right]^{2+}$ possuir $\mathrm{pK}_{\mathrm{a}}>7,50$ (Figura 37). Assim, a espécie trans-[Ru(CO) $\left.\left(\mathrm{NH}_{3}\right)_{4}\left((\mathrm{O}) \mathrm{P}(\mathrm{OH})_{2}\right)\right]^{+}$seria rapidamente consumida, o que dificultaria sua observação em solução. Após a formação da espécie trans- $\left[\mathrm{Ru}(\mathrm{CO})\left(\mathrm{NH}_{3}\right)_{4}\left((\mathrm{O}) \mathrm{P}(\mathrm{H})(\mathrm{OH})_{2}\right)\right]^{2+}$, de acordo com dados de ${ }^{31} \mathrm{P} \mathrm{RMN}$ obtidos ao longo de 65 dias, esta decai liberando fósforo em solução com $\mathrm{k}=1,75 \times 10^{-7} \mathrm{~s}^{-1}$ (Figura 37).

$$
\text { Comparando os íons trans- }\left[\mathrm{Ru}(\mathrm{NO})\left(\mathrm{NH}_{3}\right)_{4}\left(\mathrm{P}(\mathrm{O})(\mathrm{OH})_{2}\right)\right]^{2+} \quad \text { e }
$$
trans- $\left[\mathrm{Ru}(\mathrm{CO})\left(\mathrm{NH}_{3}\right)_{4}\left(\mathrm{P}(\mathrm{O})(\mathrm{OH})_{2}\right)\right]^{+}$, o carbonilo complexo é consideravelmente mais estável em solução, uma vez que, mesmo em pH 7,5 apresenta constante de decaimento de $\mathrm{k}=1,10 \times 10^{-5} \mathrm{~s}^{-1}$, ao passo que no nitrosilo decai com $\mathrm{k}=2,40 \times 10^{-4} \mathrm{~s}^{-1} \mathrm{em} \mathrm{pH} \mathrm{3,0.} \mathrm{Em}$ adição, apesar do $\mathrm{CO}$ ser um aceptor $\pi$ mais fraco que o NO, este também se mostrou capaz de induzir a isomerização do ácido fosforoso coordenado. 


\section{CONCLUSÕES}

A coordenação ao centro metálico de rutênio(II) torna os ésteres de fósforo(III) mais estáveis com respeito às reação de hidrólise dos grupos alquilas e de oxidação em meio aquoso. Esta estabilização é proporcional à extensão da retrodoação $\mathrm{Ru}(\mathrm{II}) \rightarrow \mathrm{P}(\mathrm{III})$, ou seja, é maior nos aqua do que nos nitrosilos complexos.

Todos os nitrosilos complexos de ésteres de fósforo aqui relatados liberaram rapidamente óxido nítrico $\left(\mathrm{k}_{-\mathrm{NO}}=0,22-2,87 \mathrm{~s}^{-1}\right.$, para $\mathrm{P}(\mathrm{O})(\mathrm{OH})_{2}$ e $\mathrm{P}\left(\mathrm{O}^{\mathrm{i}} \mathrm{C}_{3} \mathrm{H}_{7}\right)_{3}$ respectivamente) e/ou nitroxila ( $\left.\mathrm{k}_{-\mathrm{NO}}{ }^{-} \gg \mathrm{k}_{-\mathrm{NO}}\right)$ após serem ativados por redução eletroquímica. As velocidades de liberação de NO estão diretamente relacionada à capacidade trans labilizante do fosfito.

Os nitrosilos complexos de trialquil fosfitos hidrolisam em solução aquosa $\mathrm{pH} 3,0$ dando origem aos respectivos complexos de dialquil fosfitos, com constantes de hidrólise na faixa de $\mathrm{k}=3,00-0,29 \times 10^{-6} \mathrm{~s}^{-1}$. A correlação entre as constantes de velocidade de hidrólise e os deslocamentos químico de carbono $\left({ }^{13} \mathrm{C} \mathrm{RMN}\right)$ indica que a hidrólise do éster de fósforo coordenado ao centro metálico de rutênio(II) ocorre preferencialmente via mecanismo de Michaelis Arbusov. Uma exceção é o nitrosilo complexo trans-[Ru(NO) $\left.\left(\mathrm{NH}_{3}\right)_{4} \mathrm{P}\left(\mathrm{OCH}_{3}\right)_{3}\right]^{3+}$ que provavelmente apresenta hidrólise via mecanismo de Asknes, isto devido ao fato deste apresentar o grupo alquila com menor efeito estérico em sua estrutura e o átomo de fósforo mais positivo dentre os compostos aqui estudados.

No complexo trans- $\left[\mathrm{Ru}(\mathrm{NO})\left(\mathrm{NH}_{3}\right)_{4}\left(\mathrm{P}(\mathrm{O})(\mathrm{OH})_{2}\right)\right]^{2+}$, o nitrosônio torna o centro metálico com características de rutênio(III) o que favorece a isomerização do ligante de ácido fosforoso formando as espécies trans- $\left[\mathrm{Ru}(\mathrm{NO})\left(\mathrm{NH}_{3}\right)_{4}\left((\mathrm{O}) \mathrm{P}(\mathrm{OH})_{2}\right)\right]^{2+} \quad \mathrm{e}$ trans- $\left[\mathrm{Ru}(\mathrm{NO})\left(\mathrm{NH}_{3}\right)_{4}\left((\mathrm{O}) \mathrm{P}(\mathrm{H})(\mathrm{OH})_{2}\right)\right]^{3+}$ e só então ocorre a dissociação do ácido fosforoso.

Dados de espectroscopia eletrônica e infravermelho, unidos aos cálculos teóricos, 
mostram que o $\mathrm{CO}$ no íon complexo trans- $\left[\mathrm{Ru}(\mathrm{CO})\left(\mathrm{NH}_{3}\right)_{4}\left(\mathrm{P}(\mathrm{O})(\mathrm{OH})_{2}\right)\right]^{2+}$ também induz a isomerização do ácido fosforoso coordenado. Entretanto, devido ao fato do $\mathrm{CO}$ ser um recebedor $\pi$ mais fraco do que o $\mathrm{NO}^{+}$, a velocidade de isomerização é consideravelmente menor que no nitrosilo complexo.

$\mathrm{O}$ complexo trans- $\left[\mathrm{Ru}(\mathrm{NO})\left(\mathrm{NH}_{3}\right)_{4}\left(\mathrm{P}(\mathrm{O})\left(\mathrm{OCH}_{2} \mathrm{CH}_{3}\right)_{2}\right)\right]^{2+}$, em que o éster de fósforo coordenado é um dialquil fosfito, demonstrou elevada estabilidade em meio aquoso $(\mathrm{k}=8,90$ $\times 10^{-7} \mathrm{~s}^{-1}, \mathrm{pH} 3,0$ e $25^{\circ} \mathrm{C}$ ), sendo o mais estável dentre os compostos aqui estudados. O estudo sistemático dos nitrosilos complexos de rutênio com diferentes tipos de ésteres de fósforo mostrou que quando grupos indutores $\sigma\left(\mathrm{CH}_{2} \mathrm{CH}_{3}\right)$ estão combinados a grupos $\mathrm{OH}$ ocorre proteção do éster de fósforo com relação às reações de hidrolise e de isomerização. Em adição, ocorre também uma proteção sobre o grupamento nitrosônio reduzindo a constante de formação de nitrito complexo. Seria interessante para a obtenção de doadores de NO estáveis em meio fisiológico, dessa forma, a síntese de compostos do tipo trans- $\left[\mathrm{Ru}(\mathrm{NO})\left(\mathrm{NH}_{3}\right)_{4} \mathrm{P}(\mathrm{III})\right]^{3+}$ em que $\mathrm{P}(\mathrm{III})=\mathrm{P}(\mathrm{OH})\left(\mathrm{O}^{\mathrm{i}} \mathrm{C}_{3} \mathrm{H}_{7}\right)_{2}$ e $\mathrm{P}(\mathrm{OH})\left(\mathrm{OC}_{4} \mathrm{H}_{9}\right)_{2}$.

Os resultados aqui reunidos mostram que a coordenação ao centro metálico de rutênio(II) promove alterações consideráveis na reatividade de ésteres de fósforo(III) tornando-os mais estáveis quanto à hidrólise e à oxidação. Em adição, permitem observar que, no sistema de tetraaminas de rutênio(II), quando trans-posicionados ésteres de fósforo $\left(\mathrm{P}(\mathrm{OR})_{3}\right)$ e ligantes $\pi$-aceptores $\left(\mathrm{NO}^{+}\right.$e $\left.\mathrm{CO}\right)$ exercem mútuo efeito e influência trans. Assim, as reações de hidrólise e de isomerização do éster de fósforo coordenado podem ser moduladas em função dos grupamentos $\mathrm{R}$ dos fosfitos e da capacidade $\pi$-aceptora do ligante em posição trans. Estas informações podem ser úteis no desenvolvimento de novos complexos para aplicações biológicas e/ou catálise, além de serem importantes para o conhecimento da química de fósforo(III) de per si. 


\section{REFERÊNCIAS BIBLIOGRÁFICAS}

[1] FUKUTO, J. M.; CARRINGTON, S. J.; TANTILLO, D. J.; HARRISON, J. G.; IGNARRO, L. J.; FREEMAN, B. A.; CHEN, A.; WINK, D. A. Small molecule signaling agents: The integrated chemistry and biochemistry of nitrogen oxides, oxides of carbon, dioxygen, hydrogen sulfide, and their derived species. Chemical Research in Toxicology, v. 25, p. 769-793, 2012.

[2] LI, L.; MOORE, P. K. An overview of the biological significance of endogenous gases: new roles for old molecules. Biochemical Society Transactions, v. 35, p. 1138-1141, 2007.

[3] MUSTAFA, A. K.; GADALLA, M. M.; SNYDER, S. H. Signaling by gasotransmitters. Science Signaling, v. 2, n. 68, re2, p.1-8, 2009.

[4] WINK, D. A.; MITCHELL, J. B. Nitric oxide and cancer: an introduction. Free Radical Biology \& Medicine, v. 34, p. 951-954, 2003.

[5] FORD, P. C.; WINK, D. A.; STANBURY, D. M. Autoxidation kinetics of aqueous Nitricoxide. Febs Letters, v. 326, p. 1-3, 1993.

[6] FURCHGOTT, R. F.; ZAWADZKI, J. V. The obligatory role of endothelial-cells in the relaxation of arterial smooth-muscle by acetylcholine. Nature, v. 288, p. 373-376, 1980.

[7] PALMER, R. M. J.; FERRIGE, A. G.; MONCADA, S. Nitric-oxide release accounts for the biological-activity of endothelium-derived relaxing factor. Nature, v. 327, p. 524-526, 1987.

[8] IGNARRO, L. J.; BUGA, G. M.; WOOD, K. S.; BYRNS, R. E.; CHAUDHURI, G. Endothelium-derived relaxing factor produced and released from artery and vein is nitricoxide. Proceedings of the National Academy of Sciences of the United States of America, v. 84, p. 9265-9269, 1987.

[9] PALMER, R. M.; ASHTON, D. S.; MONCADA, S. Vascular endothelial cells synthesize nitric oxide from L-arginine. Nature, v. 333, p. 664-666, 1988.

[10] YETIK-ANACAK, G.; CATRAVAS, J. D. Nitric oxide and the endothelium: history and impact on cardiovascular disease. Vascular Pharmacology, v. 45, p. 268-276, 2006.

[11] IGNARRO, L. J. Endothelium-derived nitric-oxide - Actions and properties. Faseb Journal, v. 3, p. 31-36, 1989. 
[12] BREDT, D. S.; HWANG, P. M.; SNYDER, S. H. Localization of nitric oxide synthase indicating a neural role for nitric oxide. Nature, v. 347, p. 768-70, 1990.

[13] GARTHWAITE, J. Glutamate, nitric oxide and cell-cell signalling in the nervous system. Trends in Neurosciences, v. 14, p. 60-67, 1991.

[14] GARTHWAITE, J. Neural nitric oxide signalling. Trends in Neurosciences, v. 18, p. 51-52, 1995.

[15] HIBBS JUNIOR, J. B.; TAINTOR, R. R.; VAVRIN, Z.; RACHLIN, E. M. Nitric oxide: a cytotoxic activated macrophage effector molecule. Biochemical and Biophysical Research Communications, v. 157, p. 87-94, 1988.

[16] MARLETTA, M. A.; YOON, P. S.; IYENGAR, R.; LEAF, C. D.; WISHNOK, J. S. Macrophage oxidation of L-arginine to nitrite and nitrate: nitric oxide is an intermediate. Biochemistry, v. 27, p. 8706-8711, 1988.

[17] SILVA, J. J.; OSAKABE, A. L.; PAVANELLI, W. R.; SILVA, J. S.; FRANCO, D. W. In vitro and in vivo antiproliferative and trypanocidal activities of ruthenium $\mathrm{NO}$ donors.

British Journal of Pharmacology, v. 152, p. 112-121, 2007.

[18] IGNARRO, L. J. Nitric oxide: biology and pathobiology. Boca Raton: Academic Press, $2000.1017 \mathrm{p}$.

[19] MCCLEVERTY, J. A. Chemistry of nitric oxide relevant to biology. Chemical Reviews, v. 104, p. 403-418, 2004.

[20] MIRANDA, K. M. The chemistry of nitroxyl (HNO) and implications in biology. Coordination Chemistry Reviews, v. 249, p. 433-455, 2005.

[21] MIRANDA, K. M.; RIDNOUR, L.; ESPREY, M.; CITRIN, D.; THOMAS, D.; MANCARDI, D.; DONZELLI, S.; WINK, D. A.; KATORI, T.; TOCCHETTI, C. G.; FERLITO, M.; PAOLOCCI, N.; FUKUTO, J. M. Comparison of the chemical biology of NO and HNO: An inorganic perspective. Progress in Inorganic Chemistry, v. 54, p. 349-384, 2005 .

[22] FUKUTO, J. M.; BIANCO, C. L.; CHAVEZ, T. A. Nitroxyl (HNO) signaling. Free Radical Biology and Medicine, v. 47, p. 1318-1324, 2009. 
[23] MILLER, T. W.; CHERNEY, M. M.; LEE, A. J.; FRANCOLEON, N. E.; FARMER, P. J.; KING, S. B.; HOBBS, A. J.; MIRANDA, K. M.; BURSTYN, J. N.; FUKUTO, J. M. The effects of nitroxyl (HNO) on soluble guanylate cyclase activity interactions at ferrous heme and cysteine thiols. Journal of Biological Chemistry, v. 284, p. 21788-21796, 2009.

[24] SWITZER, C. H.; FLORES-SANTANA, W.; MANCARDI, D.; DONZELLI, S.; BASUDHAR, D.; RIDNOUR, L. A.; MIRANDA, K. M.; FUKUTO, J. M.; PAOLOCCI, N.; WINK, D. A. The emergence of nitroxyl (HNO) as a pharmacological agent. Biochimica et Biophysica Acta-Bioenergetics, v. 1787, p. 835-840, 2009.

[25] FLORES-SANTANA, W.; SWITZER, C.; RIDNOUR, L. A.; BASUDHAR, D.; MANCARDI, D.; DONZELLI, S.; THOMAS, D. D.; MIRANDA, K. M.; FUKUTO, J. M.; WINK, D. A. Comparing the chemical biology of NO and HNO. Archives of Pharmacal Research, v. 32, p. 1139-1153, 2009.

[26] IGNARO, L. J. Biosynthesis and metabolism of endothelium-derived Nitric Oxide. Annual Review of Pharmacology and Toxicology, v. 30, p. 535-560, 1990.

[27] TFOUNI, E.; TRUZZI, D. R.; TAVARES, A.; GOMES, A. J.; FIGUEIREDO, L. E.; FRANCO, D. W. Biological activity of ruthenium nitrosyl complexes. Nitric Oxide, v. 26, p. 38-53, 2012.

[28] CLARKE, M. J.; ZHU, F.; FRASCA, D. R. Non-platinum chemotherapeutic metallopharmaceuticals. Chemical Reviews, v. 99, p. 2511-2534, 1999.

[29] DYSON, P. J.; SAVA, G. Metal-based antitumour drugs in the post genomic era. Dalton Transactions, p. 1929-1933, 2006.

[30] LEVINA, A.; MITRA, A.; LAY, P. A. Recent developments in ruthenium anticancer drugs. Metallomics, v. 1, p. 458-470, 2009.

[31] BATES, J. N.; BAKER, M. T.; GUERRA, R., JR.; HARRISON, D. G. Nitric oxide generation from nitroprusside by vascular tissue. Evidence that reduction of the nitroprusside anion and cyanide loss are required. Biochemical Pharmacology, v. 42, p. 157-165, 1991.

[32] FORESTIER, J. Rheumatoid arthritis and its treatment by gold salts - The results of six years experience. Journal of Laboratory and Clinical Medicine, v. 20, p. 827-840, 1935. 
[34] FRICKER, S. P. Metal based drugs: from serendipity to design. Dalton Transactions, p. 4903-4917, 2007.

[35] STOCHEL, G.; WANAT, A.; KULIS, E.; STASICKA, Z. Light and metal complexes in medicine. Coordination Chemistry Reviews, v. 171, p. 203-220, 1998.

[36] MONCADA, S.; PALMER, R. M. J.; HIGGS, E. A. Nitric-Oxide - physiology, pathophysiology, and pharmacology. Pharmacological Reviews, v. 43, p. 109-142, 1991.

[37] PEACOCK, A. F. A.; PARSONS, S.; SADLER, P. J. Tuning the hydrolytic aqueous chemistry of osmium arene complexes with N,O-chelating ligands to achieve cancer cell cytotoxicity. Journal of the American Chemical Society, v. 129, p. 3348-3357, 2007.

[38] SAVA, G.; CAPOZZI, I.; CLERICI, K.; GAGLIARDI, G.; ALESSIO, E.; MESTRONI, G. Pharmacological control of lung metastases of solid tumours by a novel ruthenium complex. Clinical \& Experimental Metastasis, v. 16, p. 371-379, 1998.

[39] DWYER, F. P.; GYARFAS, E. C.; ROGERS, W. P.; KOCH, J. H. Biological activity of complex ions. Nature, v. 170, p. 190-191, 1952.

[40] BERGAMO, A.; SAVA, G. Ruthenium complexes can target determinants of tumour malignancy. Dalton Transactions, p. 1267-1272, 2007.

[41] SAVA, G.; BERGAMO, A.; ZORZET, S.; GAVA, B.; CASARSA, C.; COCCHIETTO, M.; FURLANI, A.; SCARCIA, V.; SERLI, B.; IENGO, E.; ALESSIO, E.; MESTRONI, G. Influence of chemical stability on the activity of the antimetastasis ruthenium compound NAMI-A. European Journal of Cancer, v. 38, p. 427-435, 2002.

[42] HARTINGER, C. G.; JAKUPEC, M. A.; ZORBAS-SEIFRIED, S.; GROESSL, M.; EGGER, A.; BERGER, W.; ZORBAS, H.; DYSON, P. J.; KEPPLER, B. K. KP1019, a new redox-active anticancer agent - preclinical development and results of a clinical phase I study in tumor patients. Chemistry \& Biodiversity, v. 5, p. 2140-2155, 2008.

[43] OSTI, R. Z.; SERRANO, F. A.; PASCHOALIN, T.; MASSAOKA, M.; TRAVASSOS, L. R.; TRUZZI, D. R.; RODRIGUES, E. G.; FRANCO, D. W. The in vitro and in vivo antitumor activities of nitrosyl ruthenium am(m)ine complexes against murine and human tumor cell lines. Australian Journal of Chemistry, v. 65, p. 1333-1341, 2012. 
[44] TFOUNI, E.; DORO, F. G.; FIGUEIREDO, L. E.; PEREIRA, J. C. M.; METZKER, G.; FRANCO, D. W. Tailoring NO donors metallopharmaceuticals: Ruthenium nitrosyl ammines and aliphatic tetraazamacrocycles. Current Medicinal Chemistry, v. 17, p. 3643-3657, 2010 .

[45] TFOUNI, E.; KRIEGER, M.; MCGARVEY, B. R.; FRANCO, D. W. Structure, chemical and photochemical reactivity and biological activity of some ruthenium amine nitrosyl complexes. Coordination Chemistry Reviews, v. 236, p. 57-69, 2003.

[46] TOLEDO JUNIOR, J. C.; LIMA NETO, B. D. S. ; FRANCO, D. W. Mutual effects in the chemical properties of the ruthenium metal center and ancillary ligands upon coordination. Coordination Chemistry Reviews, v. 249, p. 419-431, 2005.

[47] TFOUNI, E.; DORO, F. G.; GOMES, A. J.; SILVA, R. S. D.; METZKER, G.; BENINI, P. G. Z.; FRANCO, D. W. Immobilized ruthenium complexes and aspects of their reactivity. Coordination Chemistry Reviews, v. 254, p. 355-371, 2010.

[48] JONES, D. P.; GO, Y. M.; ANDERSON, C. L.; ZIEGLER, T. R.; KINKADE, J. M.; KIRLIN, W. G. Cysteine/cystine couple is a newly recognized node in the circuitry for biologic redox signaling and control. Faseb Journal, v. 18, p. 1246-1248, 2004.

[49] MILLIS, K. K.; WEAVER, K. H.; RABENSTEIN, D. L. Oxidation-reduction potential of glutathione. Journal of Organic Chemistry, v. 58, p. 4144-4146, 1993.

[50] OPRIAN, D. D.; COON, M. J. Oxidation-reduction states of FMN and FAD in NADPHCytochrome-P-450 reductase during reduction by NADPH. Journal of Biological

Chemistry, v. 257, p. 8935-8944, 1982.

[51] LOPES, L. G. F.; CASTELLANO, E. E.; FERREIRA, A. G.; DAVANZO, C. U.; CLARKE, M. J.; FRANCO, D. W. Reactivity of trans- $\left[\mathrm{Ru}\left(\mathrm{NH}_{3}\right)_{4} \mathrm{P}(\mathrm{OEt})_{3} \mathrm{NO}\right] \mathrm{X}_{3}\left(\mathrm{X}=\mathrm{PF}_{6}^{-}\right.$, $\mathrm{CF}_{3} \mathrm{COO}^{-}$): modulation of the release of $\mathrm{NO}$ by the trans-effect. Inorganica Chimica Acta, v. 358, p. 2883-2890, 2005.

[52] METZKER, G.; STEFANELI, E. V.; PEREIRA, J. C. M.; LIMA, F. D. A.; DA SILVA, S. C.; FRANCO, D. W. Nitric oxide and nitroxyl formation in the reduction of transtetraamminenitrosyltriethylphosphiteruthenium(II) ion. Inorganica Chimica Acta, v. 394, p. 765-769, 2013.

[53] PLICAS, L. M. A.; FRANCO, D. W. On the Reaction of Carbon-Monoxide with the Complexes trans- $\left[\mathrm{Ru}\left(\mathrm{NH}_{3}\right)_{4} \mathrm{P}(\mathrm{OEt})_{3}\left(\mathrm{H}_{2} \mathrm{O}\right)\right]^{2+}$ and trans- $\left[\mathrm{Ru}\left(\mathrm{NH}_{3}\right)_{4} \mathrm{Pph}_{3}\left(\mathrm{H}_{2} \mathrm{O}\right)\right]^{2+}$. Inorganica Chimica Acta, v. 134, p. 265-268, 1987. 
[54] FILHO, J. C. D. N.; LIMA, J. B. D.; LIMA NETO, B. D. S.; FRANCO, D. W. Transeffect and trans-influence series for phosphanes in octahedral environment: a guide for design of new catalysts Journal of Molecular Catalysis, v. 90, p. 257-266, 1994.

[55] GUTHRIE, J. P. Tautomerization equilibria for phosphorus acid and its ethyl-esters, freeenergies of formation of phosphorus and phosphonic acids and their ethyl-esters, and $\mathrm{pKa}$ values for ionization of the P-H bond in phosphonic acid and phosphonic esters.

Canadian Journal of Chemistry, v. 57, p. 236-239, 1979.

[56] SERNAGLIA, R. L.; FRANCO, D. W. The ruthenium(II) center and the phosphite phosphonate tautomeric equilibrium. Inorganic Chemistry, v. 28, p. 3485-3489, 1989.

[57] AKBAYEVA, D. N.; DI VAIRA, M.; COSTANTINI, S. S.; PERUZZINI, M.;

STOPPIONI, P. Stabilization of the tautomers $\mathrm{HP}(\mathrm{OH})_{2}$ and $\mathrm{P}(\mathrm{OH})_{3}$ of hypophosphorous and phosphorous acids as ligands. Dalton Transactions, p. 389-395, 2006.

[58] DI VAIRA, M.; PERUZZINI, M.; STOPPIONI, P. $\mathrm{d}^{6}$ metal systems for white phosphorus activation. Comptes Rendus Chimie, v. 13, p. 935-942, 2010.

[59] TRUZZI, D. R.; FERREIRA, A. G.; DA SILVA, S. C.; CASTELLANO, E. E.; CHAGAS ALVES LIMA, F. D.; FRANCO, D. W. Nitrosyl induces phosphorous-acid dissociation in ruthenium(II). Dalton Transactions, v. 40, p. 12917-12925, 2011.

[60] KIRBY, A. J.; WARREN, S. G. The organic chemistry of phosphorus. Amsterdam: Elsevier , 1967. 404 p.

[61] MCINTYRE, S. K.; ALAM, T. M. O-17 NMR investigation of phosphite hydrolysis mechanisms. Magnetic Resonance in Chemistry, v. 45, p. 1022-1026, 2007.

[62] BHATTACHARYA, A. K.; THYAGARAJAN, G. The Michaelis-Arbuzov rearrangement. Chemical Reviews, v. 81, p. 415-430, 1981.

[63] AKSNES, G.; AKSNES, D. Kinetics mechanism of reaction of tripropyl phosphite with water in acetonitrile. Acta Chemica Scandinavica, v. 18, p. 1623-1628, 1964.

[64] AKSNES, G.; AKSNES, D. Kinetic study of Michaelis-Arbuzov reactions. Acta Chemica Scandinavica, v. 18, p. 38-46, 1964. 
[65] MITCHELL, M. C.; TAYLOR, R. J.; KEE, T. P. On the hydrolysis of dimethyl-Hphosphonate. An O-18-labelling and P-31-NMR study. Polyhedron, v. 17, p. 433-442, 1998.

[66] FRANCO, D. W. The aquation and trans-influence of phosphites in complexes of ruthenium(II). Inorganica Chimica Acta, v. 48, p. 1-7, 1981.

[67] FRANCO, D. W.; TAUBE, H. Triethyl phosphite as a ligand on ruthenium(II). Inorganic Chemistry, v. 17, p. 571-578, 1978.

[68] REZENDE, N. M. S.; MARTINS, S. D.; MARINHO, L. A.; DOSSANTOS, J. A. V.; TABAK, M.; PERUSSI, J. R.; FRANCO, D. W. Triethyl phosphite as a ligand on Ru(III) Synthesis, characterization and properties of trans- $\left[\mathrm{Ru}\left(\mathrm{NH}_{3}\right)_{4} \mathrm{P}(\mathrm{OEt})_{3}\left(\mathrm{H}_{2} \mathrm{O}\right)\right]\left(\mathrm{CF}_{3} \mathrm{SO}_{3}\right)_{3}$. Inorganica Chimica Acta, v. 182, p. 87-92, 1991.

[69] METZKER, G.; TOLEDO JUNIOR., J. C.; LIMA, F. C. A.; MAGALHÃES, A.; CARDOSO, D. R.; FRANCO, D. W. Nitric oxide as an activation agent for nucleophilic attack in trans-[Ru(NO) $\left.\left(\mathrm{NH}_{3}\right)_{4}\left\{\mathrm{P}(\mathrm{OEt})_{3}\right\}\right]\left(\mathrm{PF}_{6}\right)_{3}$. Journal of Brazilian Chemical Society, $\mathrm{v}$. 21, p. 1266-1273, 2010.

[70] XI, C. J.; LIU, Y. H.; LAI, C. B.; ZHOU, L. H. Synthesis of molybdenum complex with novel $\mathrm{P}(\mathrm{OH})_{3}$ ligand based on the one-pot reaction of $\mathrm{Mo}(\mathrm{CO})_{6}$ with $\mathrm{HP}(\mathrm{O})(\mathrm{OEt})_{2}$ and water. Inorganic Chemistry Communications, v. 7, p. 1202-1204, 2004.

[71] PERRIN, D. D.; ALMARGE, W., L.; PERRIN, D. P. Purification of laboratory chemicals. New York: Butterworth Heinemann, 1983. 529 p.

[72] BAILAR JUNIOR, J. C. Inorganic syntheses. New York: McGraw W-Hill, 1953. p. 5860 .

[73] SHRIVER, D. F. The manipulation of air-sensitive compounds. New York: McGrawHill, 1969. 299 p.

[74] ALLEN, A. D.; BOBMTEY, F.; HARRIS, R. D.; REINSTAU, U. P.; SENAFF, C. V. Inorganic syntheses. New York: McGraw-Hill, 1972. p. 2-4.

[75] CLARKE, M. J. Electrochemistry, synthesis, and spectra of pentaammineruthenium(III) complexes of cytidine, adenosine, and related ligands. Journal of the American Chemical Society, v. 100, p. 5068-5075, 1978. 
[76] TOLEDO JUNIOR, J. C. Aspectos da reatividade de complexos de rutênio contendo óxido nítrico como ligante. 2004, 119 f. Tese (Doutorado em Química Analítica) - Instituto de Química, Universidade de São Paulo, São Carlos, 2004.

[77] NICHOLSON, R. S.; SHAIN, I. Theory of stationary electrode polarography - Single scan and cyclic methods applied to reversible, irreversible, and kinetic systems. Analytical Chemistry, v. 36, p. 706-723, 1964.

[78] ENRAF-NONIUS; Collect, Nonius BV, The Netherlands, 2000. Programa de computador.

[79] OTWINOWSKI, Z.; MINOR, W.; DENZO, H. K. L. Methods in enzymology. New York: Elsevier, 1997. v. 278.

[80] COPPENS, P.; LEISERO, W. L.; RABINOV, I. D. Calculation of absorption corrections for camera and diffractometer data. Acta Crystallographica, v. 18, p. 1035-1038, 1965.

[81] SHELDRICK, G. M. Program for crystal structure refinement: SHELXS97. Gottingen, 1997. Programa de computador.

[82] SHELDRICK, G. M. Program for crystal structure analysis: SHELXS97. Gottingen, 1997. Programa de computador.

[83] FARRUGIA, L. J. ORTEP-3 for Windows - a version of ORTEP-III with a Graphical User Interface (GUI). Journal of Applyed Crystallography, v. 30, p. 565, 1997. 
[84] FRISCH, M. J.; TRUCKS, G. W.; SCHLEGEL, H. B.; SCUSERIA, G. E.; ROBB, M. A.; CHEESEMAN, J. R.; MONTGOMERY JR., J. A.; VREVEN, T.; KUDIN, K. N.; BURANT, J. C.; MILLAM, J. M.; IYENGAR, S. S.; TOMASI, J.; BARONE, V.; MENNUCCI, B.; COSSI, M.; SCALMANI, G.; REGA, N.; PETERSSON, G. A.; NAKATSUJI, H.; HADA, M.; EHARA, M.; TOYOTA, K.; FUKUDA, R.; HASEGAWA, J.; ISHIDA, M.; NAKAJIMA, T.; HONDA, Y.; KITAO, O.; NAKAI, H.; KLENE, M.; LI, X.; KNOX, J. E.; HRATCHIAN, H. P.; CROSS, J. B.; BAKKEN, V.; ADAMO, C.; JARAMILLO, J.; GOMPERTS, R.; STRATMANN, R. E.; YAZYEV, O.; AUSTIN, A. J.; CAMMI, R.; POMELLI, C.; OCHTERSKI, J. W.; AYALA, P. Y.; MOROKUMA, K.; VOTH, G. A.; SALVADOR, P.; DANNENBERG, J. J.; ZAKRZEWSKI, V. G.; DAPPRICH, S.; DANIELS, A. D.; STRAIN, M. C.; FARKAS, O.; MALICK, D. K.; RABUCK, A. D.; RAGHAVACHARI, K.; FORESMAN, J. B.; ORTIZ, J. V.; CUI, Q.; BABOUL, A. G.; CLIFFORD, S.; CIOSLOWSKI, J.; STEFANOV, B. B.; LIU, G.; LIASHENKO, A.; PISKORZ, P.; KOMAROMI, I.; MARTIN, R. L.; FOX, D. J.; KEITH, T.; AL-LAHAM, M. A.; PENG, C. Y.; NANAYAKKARA, A.; CHALLACOMBE, M.; GILL, P. M. W.; JOHNSON, B.; CHEN, W.; WONG, M. W.; GONZALEZ, C.; POPLE, J. A. Gaussian: version 09, Wallingford, 2004. Programa de computador.

[85] KOHN, W.; SHAM, L. J. Self-consistent equations including exchange and correlation effects. Physical Review, v. 140, p. 1133-1138, 1965.

[86] BECKE, A. D. A New mixing of Hartree-Fock and local Density-Functional Theories. Journal of Chemical Physics, v. 98, p. 1372-1377, 1993.

[87] LEE, C.; YANG, W.; PARR, R. G. Development of the colle-salvetti correlation-energy formula into a functional of the electron density. Physical Review B, v. 37, p. 785-789, 1988.

[88] GODBOUT, N.; SALAHUB, D. R.; ANDZELM, J.; WIMMER, E. Optimization of Gaussian-type basis-sets for local spin-density functional calculations. Boron through Neon, optimization technique and validation. Canadian Journal of Chemistry, v. 70, p. 560-571, 1992.

[89] CANCES, E.; MENNUCCI, B.; TOMASI, J. A new integral equation formalism for the polarizable continuum model: Theoretical background and applications to isotropic and anisotropic dielectrics. Journal of Chemical Physics, v. 107, p. 3032-3041, 1997.

[90] REED, A. E.; WEINHOLD, F. Natural localized molecular-orbitals. Journal of Chemical Physics, v. 83, p. 1736-1740, 1985.

[91] REED, A. E.; CURTISS, L. A.; WEINHOLD, F. Intermolecular interactions from a natural bond orbital, donor-acceptor viewpoint. Chemical Reviews, v. 88, p. 899-926, 1988. 
[92] TRUZZI, D. R. Comportamento em solução de $\mathbf{P}(\mathbf{O H})(\mathrm{OEt})_{2} \mathbf{e} \mathbf{P}(\mathbf{O H})_{3}$ coordenados a tetraaminas de Ru(II). 2010. 94 f. Dissertação (Mestrado em Química Analítica) - Instituto de Química de São Carlos, Universidade de São Paulo, São Carlos, 2010.

[93] ALGARRA, A. G.; FERNANDEZ-TRUJILLO, M. J.; HERNANDEZ-MOLINA, R.; BASALLOTE, M. G. Kinetic, DFT and TD-DFT studies on the mechanism of stabilization of pyramidal $\mathrm{H}(3) \mathrm{PO}(3)$ at the $\left[\mathrm{Mo}(3) \mathrm{M}^{\prime} \mathrm{S}(4)(\mathrm{H}(2) \mathrm{O})(10)\right](4+)$ clusters $\left(\mathrm{M}^{\prime}=\mathrm{Pd}\right.$, Ni). Dalton Transaction, v. 40, p. 8589-8597, 2011.

[94] SILVA, W. C.; CASTELLANO, E. E.; FRANCO, D. W. Structural and reactivity changes in ruthenium ammines induced by the NO ligand. Polyhedron, v. 23, p. 1063-1067, 2004.

[95] BEZERRA, C. W. B.; DA SILVA, S. C.; GAMBARDELLA, M. T. P.; SANTOS, R. H. A.; PLICAS, L. M. A.; TFOUNI, E.; FRANCO, D. W. Water pi-donation in trans-tetraammineruthenium(II): Effect on coordinated-water properties induced by a trans NO ligand. Inorganic Chemistry, v. 38, p. 5660-5667, 1999.

[96] YEH, A.; SCOTT, N.; TAUBE, H. S to O and O to S linkage isomerization in sulfoxide complexes of pentaammine-ruthenium. Inorganic Chemistry, v. 21, p. 2542-2545, 1982.

[97] JIANG, M. L.; DALGARNO, S.; KILNER, C. A.; HALCROW, M. A.; KEE, T. P. Chiral bis(oxazoline) complexes. Synthesis, structure and applications in catalytic phosphotransfer. Polyhedron, v. 20, p. 2151-2162, 2001.

[98] DAVIES, S. R.; MITCHELL, M. C.; CAIN, C. P.; DEVITT, P. G.; TAYLOR, R. J.; KEE, T. P. Phospho-transfer catalysis - On the asymmetric hydrophosphonylation of aldehydes. Journal of Organometallic Chemistry, v. 550, p. 29-57, 1998.

[99] CARLOS, R. M.; FERRO, A. A.; SILVA, H. A. S.; GOMES, M. G.; BORGES, S. S. S.; FORD, P. C.; TFOUNI, E.; FRANCO, D. W. Photochemical reactions of trans- $\left[\mathrm{Ru}\left(\mathrm{NH}_{3}\right)_{4} \mathrm{~L}(\mathrm{NO})\right]^{3+}$ complexes. Inorganica Chimica Acta, v. 357, p. 1381-1388, 2004.

[100] GORELSKY, S. I.; DA SILVA, S. C.; LEVER, A. B. P.; FRANCO, D. W. Electronic spectra of trans- $\left[\mathrm{Ru}\left(\mathrm{NH}_{3}\right)_{4}(\mathrm{~L}) \mathrm{NO}\right]^{3+/ 2+}$ complexes. Inorganica Chimica Acta, v. 300, p. 698$708,2000$.

[101] DE OSTI, R. Z.; FRANCO, D. W. Aspects of nitrite association with trans-[Ru(NH$\left.)_{4} \mathrm{P}(\mathrm{OEt})_{3} \mathrm{H}_{2} \mathrm{O}\right]^{2+}$. Polyhedron, v. 26, p. 4746-4750, 2007. 
[102] HAMMOND, P. R. Ionisation of P-H bond - deuterium-exchange studies with diethyl and ethyl hydrogen phosphonate. Journal of the Chemical Society, p. 1365-1369, 1962.

[103] STOPPIONI, P.; AKBAYEVA, D. N.; DI VAIRA, M.; COSTANTINI, S. S.;

PERUZZINI, M. Stabilization of the tautomers $\mathrm{HP}(\mathrm{OH})(2)$ and $\mathrm{P}(\mathrm{OH})(3)$ of hypophosphorous and phosphorous acids as ligands. Dalton Transactions, p. 389-395, 2006.

[104] BRILL, T. B.; LANDON, S. J. Arbuzov-like dealkylation reactions of transition-metal phosphite complexes. Chemical Reviews, v. 84, p. 577-585, 1984.

[105] FRANCO, D. W. Ruthenium ammine complexes of group Vb donor ligands.

Coordination Chemistry Reviews, v. 119, p. 199-225, 1992.

[106] MAZZETTO, S. E.; PLICAS, L. M. D.; TFOUNI, E.; FRANCO, D. W. Photochemical behavior of trans- $\left[\mathrm{Ru}\left(\mathrm{NH}_{3}\right)_{4} \mathrm{P}(\mathrm{OEt})_{3} \mathrm{~L}\right]^{2+}$ complex-ions $\left(\mathrm{L}=\mathrm{P}(\mathrm{OEt})_{3}, \mathrm{CO}, \mathrm{H}_{2} \mathrm{O}\right)$. Inorganic Chemistry, v. 31, p. 516-519, 1992.

[107] ISIED, S. S.; TAUBE, H. Rates of substitution in cis and trans ruthenium(II) aquotetraammines. Inorganic Chemistry, v. 15, p. 3070-3075, 1976. 